\title{
S3-Leitlinie - Diagnostik und Therapie der Plattenepithelkarzinome und Adenokarzinome des Ösophagus
}

\author{
Langversion 2.0 - Dezember 2018 AWMF-Registernummer: 021/0230L
}

\author{
Authors \\ und die Mitarbeiter der Leitlinienkommission \\ Collaborators: \\ Frederick Wenz, Martin Werner \\ Institute \\ 1 Klinik für Innere Medizin, Klinikum Bremen Ost, Bremen \\ 2 Medizinische Klinik II, Klinikum Aschaffenburg, \\ Aschaffenburg \\ 3 Klinik und Poliklinik für Viszeralchirurgie, \\ Universitätsklinikum, Leipzig \\ 4 Klinik für Innere Medizin, Allgemeines Krankenhaus, Celle \\ 5 Klinik für Allgemein- und Viszeralchirurgie, \\ Agaplesion Markus Krankenhaus, Frankfurt \\ 6 Deutsche Gesellschaft für Verdauungs- und \\ Stoffwechselkrankheiten, Berlin \\ 7 Magen-Darm-Zentrum, Hamburg-Eppendorf \\ 8 Klinik für Gastroenterolgie und Interventionelle \\ Endoskopie, Krankenhaus Barmherzige Brüder, \\ Regensburg \\ 9 Klinik für Internistische Onkologie und Hämatologie, \\ Kliniken Essen-Mitte, Essen
}

Rainer Porschen ${ }^{1}$, Wolfgang Fischbach ${ }^{2}$, Ines Gockel ${ }^{3}$, Stephan Hollerbach ${ }^{4}$, Arnulf Hölscher ${ }^{5}$, Petra Lynen Jansen ${ }^{6}$, Stephan Miehlke ${ }^{7}$, Oliver Pech ${ }^{8}$, Michael Stahl ${ }^{9}$, Peter Thuss-Patience ${ }^{10}$, Udo Vanhoefer ${ }^{11}$

Gustavo Baretton, Christian Ell, Ute Goerling, Lars Grenacher, Stephan Hollerbach, Barbara Kade, Wolfram Trudo Knoefel, Jürgen Körber, Philipp Lenz, Florian Lordick, Dietmar Lorenz, Sylvie Lorenzen, Alexander Meining, Josef Menzel, Helmut Messmann, Hans-Joachim Meyer, Stefan Paul Mönig, Ute Nöthlings, Heinz Schmidberger, Matthias Schmidt, Thomas Seufferlein, Maria Steingräber, Martin Stuschke, Reina Tholen, Jörg Trojan, Christoph Wagener, Arved Weimann,

10 Klinik für Hämatologie und Onkologie,

Charité Campus Virchow Klinikum, Berlin

11 Zentrum für Innere Medizin, Marienkrankenhaus, Hamburg

Bibliografie

DOI https://doi.org/10.1055/a-0833-5712

Z Gastroenterol 2019; 57: 336-418

(c) Georg Thieme Verlag KG, Stuttgart · New York

ISSN 0044-2771

Korrespondenzadresse

Prof. Dr. med. Rainer Porschen

Chefarzt der Klinik für Innere Medizin, Klinikum Bremen Ost,

Züricher Str. 40, D-28325 Bremen

Tel.: ++ 49/421/4081221

Fax: ++49/421/4082234

rainer.porschen@klinikum-bremen-ost.de

\section{Wesentliche Neuerungen durch die Aktua- lisierung der Leitlinie (Version 2.0, 2018)}

In Rahmen der Aktualisierung wurden alle Empfehlungen auf Aktualität geprüft. Hierzu erfolgte eine systematische Recherche für priorisierte Themen sowie eine Befragung der beteiligten Fachexperten. Im Rahmen des Aktualisierungsprozesses wurden die unten aufgeführten Empfehlungen überarbeitet. Hierbei wurden teilweise lediglich die Einschätzungen bzgl. der Evidenzklassifikation aufgrund neuer Studiendaten verändert (mit einem * markiert). Eine detaillierte Übersicht der Änderungen befindet sich in > Tab. 15.

*Statement 4.2. (Alkohol als Risikofaktor)

*Statement 4.7. (Gastroösophagealer Reflux als Risikofaktor)
Empfehlungen 6.4.und 6.5. (Chromoendoskopie (Lugol'sche Lösung) oder computergestützte digitale (Filter-)Verfahren als erweiterte Diagnostik)

*Empfehlung 6.6. (Endoskopischer Ultraschall als Bestandteil des Stagings)

Empfehlung 6.13. (PET/CT bei lokal fortgeschrittenen Tumoren) Empfehlung 6.16. (Diagnostische Laparoskopie)

Empfehlung 6.18. (Pathologische Zweitmeinung bei histologischer Diagnose einer IEN/Dysplasie)

*Empfehlung 6.23. (Aussagen zum Regressions-Score im pathologischen Befund)

Empfehlung 8.10. (Resektionsausmaß) 


\begin{tabular}{|c|c|c|}
\hline \multicolumn{2}{|c|}{ Inhaltsverzeichnis } & Seite \\
\hline \multicolumn{2}{|c|}{$\begin{array}{l}\text { Wesentliche Neuerungen durch die Aktualisierung der } \\
\text { Leitlinie (Version 2.0, 2018) }\end{array}$} & 336 \\
\hline 1. & Informationen zu dieser Leitlinie & 338 \\
\hline 1.1 & Herausgeber & 338 \\
\hline 1.2. & Federführende Fachgesellschaft & 338 \\
\hline 1.3. & Finanzierung der Leitlinie & 338 \\
\hline 1.4. & Kontakt & 338 \\
\hline 1.5. & Zitierweise & 338 \\
\hline 1.6. & Besonderer Hinweis & 338 \\
\hline 1.7. & Ziele des Leitlinienprogramms Onkologie & 338 \\
\hline 1.8. & Weitere Dokumente zu dieser Leitlinie & 339 \\
\hline 1.9. & Zusammensetzung der Leitliniengruppe & 339 \\
\hline 1.9.1. & Leitlinienkoordination & 339 \\
\hline 1.9.2. & Autoren der Leitlinie & 339 \\
\hline 1.9.3. & $\begin{array}{l}\text { Beteiligte Fachgesellschaften und } \\
\text { Organisationen }\end{array}$ & 340 \\
\hline 1.9.4. & Arbeitsgruppen und Steuergruppe & 340 \\
\hline 1.9.5. & Patientenbeteiligung & 340 \\
\hline 1.9.6. & Methodische Begleitung & 340 \\
\hline 1.10. & Verwendete Abkürzungen & 340 \\
\hline 2. & Einführung & 342 \\
\hline 2.1. & Geltungsbereich und Zweck & 342 \\
\hline 2.1.1. & Zielsetzung und Fragestellung & 342 \\
\hline 2.1 .2 & Adressaten & 342 \\
\hline 2.1 .3 & Gültigkeitsdauer und Aktualisierungsverfahren & 342 \\
\hline 2.2 & Grundlagen der Methodik & 342 \\
\hline 2.2.1. & $\begin{array}{l}\text { Schema der Evidenzgraduierung nach Oxford } \\
\text { (Version 2009) }\end{array}$ & 342 \\
\hline 2.2 .2 & Schema der Empfehlungsgraduierung & 342 \\
\hline 2.2.3. & Statements & 343 \\
\hline 2.2 .4 & Expertenkonsens (EK) & 343 \\
\hline 2.2 .5 & $\begin{array}{l}\text { Unabhängigkeit und Darlegung möglicher } \\
\text { Interessenkonflikte }\end{array}$ & 344 \\
\hline 3. & Patienteninformation und Aufklärung & 344 \\
\hline 3.1. & Informationsmaterial & 344 \\
\hline 3.2 . & $\begin{array}{l}\text { Grundprinzipien einer patientenzentrierten } \\
\text { Kommunikation }\end{array}$ & 345 \\
\hline 3.3. & Therapieaufklärungsgespräch & 345 \\
\hline 4. & Risikofaktoren & 346 \\
\hline 4.1. & Rauchen & 346 \\
\hline 4.2. & Alkohol & 347 \\
\hline 4.3. & Übergewicht & 347 \\
\hline 4.4. & Weitere Risikofaktoren & 348 \\
\hline 5. & Prävention & 351 \\
\hline 6. & Primärdiagnostik und Staging inklusive Pathologie & 352 \\
\hline 6.1. & Primärdiagnostik & 352 \\
\hline 6.2 . & Erweiterte Diagnostik & 352 \\
\hline 6.3. & Staging des Ösophaguskarzinoms & 354 \\
\hline
\end{tabular}

\begin{tabular}{|c|c|c|}
\hline \multicolumn{2}{|c|}{ Inhaltsverzeichnis } & \multirow{2}{*}{$\begin{array}{l}\text { Seite } \\
361\end{array}$} \\
\hline 6.4 . & $\begin{array}{l}\text { Diagnostische Laparoskopie und Thorakoskopie } \\
\text { (Staging) }\end{array}$ & \\
\hline 6.5. & Pathologie & 362 \\
\hline 7. & Ernährungsmedizinische Versorgung & 367 \\
\hline 8. & Kurativ intendierte Therapie & 367 \\
\hline 8.1. & Allgemeine Therapieentscheidung & 367 \\
\hline 8.2. & Endoskopische Therapie & 368 \\
\hline 8.2.1. & $\begin{array}{l}\text { Endoskopische Resektion (ER) und lokal ablative } \\
\text { Verfahren }\end{array}$ & 368 \\
\hline 8.2.2. & $\begin{array}{l}\text { Vorgehen bei Lokalrezidiven nach endoskopi- } \\
\text { scher Therapie }\end{array}$ & 370 \\
\hline 8.2.3. & Nachsorge nach endoskopischer Therapie & 370 \\
\hline 8.3. & Chirurgische Therapie & 371 \\
\hline 8.3.1. & Hospitalvolumen & 371 \\
\hline 8.3.2. & Präoperative Risikoanalyse & 371 \\
\hline 8.3.3. & Chirurgische Technik & 371 \\
\hline 8.3.4. & Vorgehen bei Oligometastasierung & 375 \\
\hline 8.3.5. & Perioperative Ernährung & 376 \\
\hline 8.3.6. & Vorgehen bei R1 / R2-Resektion & 378 \\
\hline 8.3.7. & Vorgehen bei Lokalrezidiv nach Operation & 378 \\
\hline 8.4. & Multimodale Therapiekonzepte & 379 \\
\hline 8.4.1. & Präoperative Radiotherapie & 380 \\
\hline 8.4.2. & $\begin{array}{l}\text { Präoperative Radiochemotherapie und } \\
\text { perioperative Chemotherapie }\end{array}$ & 381 \\
\hline 8.4.3. & $\begin{array}{l}\text { Restaging nach präoperativer multimodaler } \\
\text { Therapie }\end{array}$ & 385 \\
\hline 8.4.4. & Responseprädiktion & 385 \\
\hline 8.4.5. & Indikation zur definitiven Radiochemotherapie & 386 \\
\hline 8.4.6. & $\begin{array}{l}\text { Vorgehen bei Tumorpersistenz/Lokalrezidiv nach } \\
\text { Radiochemotherapie }\end{array}$ & 388 \\
\hline 8.4.7. & $\begin{array}{l}\text { Stellenwert von gezielten Therapien } \\
\text { (targeted therapy) }\end{array}$ & 389 \\
\hline 8.4.8. & $\begin{array}{l}\text { Stellenwert der postoperativen adjuvanten } \\
\text { Radiotherapie oder Radiochemotherapie }\end{array}$ & 389 \\
\hline 9. & Palliativtherapie & 392 \\
\hline 9.1. & Palliative Chemotherapie: Erstlinientherapie & 392 \\
\hline 9.2. & Palliative Chemotherapie: Zweitlinientherapie & 393 \\
\hline 9.3. & Stellenwert der „Targeted Therapy“ & 394 \\
\hline 9.4 . & Palliative Radio(chemo)therapie & 395 \\
\hline 9.5 . & Palliative Brachytherapie & 395 \\
\hline 9.6. & Endoskopische Stentapplikation & 395 \\
\hline 9.7. & Stellenwert der intraluminalen lokalen Therapie & 396 \\
\hline 10. & Psychoonkologie & 397 \\
\hline 11. & Palliativversorgung & 397 \\
\hline 12. & Qualitätsindikatoren & 398 \\
\hline 13. & Anhänge & 402 \\
\hline 13.1. & Übersicht der Änderungen in Version 2.0 (2018) & 402 \\
\hline 14. & Abbildungsverzeichnis & 403 \\
\hline 15. & Tabellenverzeichnis & 403 \\
\hline Litera & zeichnis & 403 \\
\hline
\end{tabular}




\section{Informationen zu dieser Leitlinie}

\subsection{Herausgeber}

Leitlinienprogramm Onkologie der Arbeitsgemeinschaft der Wissenschaftlichen Medizinischen Fachgesellschaften (AWMF), Deutschen Krebsgesellschaft (DKG) und Deutschen Krebshilfe (DKH).

\subsection{Federführende Fachgesellschaft}

Deutsche Gesellschaft für Gastroenterologie, Verdauungs- und Stoffwechselkrankheiten (DGVS)

\section{DGVS}

Deutsche Gesellschaft für Gastroenterologie, Verdauungs- und Stoffwechselkrankheiten

\subsection{Finanzierung der Leitlinie}

Die Erstellung und kontinuierliche Aktualisierung dieser Leitlinie wurde von der Deutschen Krebshilfe im Rahmen des Leitlinienprogramms Onkologie gefördert.

\subsection{Kontakt}

Office Leitlinienprogramm Onkologie c/o

Deutsche Krebsgesellschaft e. V.

Kuno-Fischer-Straße 8

14057 Berlin

leitlinienprogramm@krebsgesellschaft.de

www.leitlinienprogramm-onkologie.de

\subsection{Zitierweise}

Leitlinienprogramm Onkologie (Deutsche Krebsgesellschaft, Deutsche Krebshilfe, AWMF): S3-Leitlinie Diagnostik und Therapie der Plattenepithelkarzinome und Adenokarzinome des Ösophagus, Langversion 2.0, 2018, AWMF-Registernummer: 021/023OL https://www.leitlinienprogramm-onkologie.de/leitlinien/ oesophaguskarzi-nom/ (abgerufen am: TT.MM.JJJ))

\subsection{Besonderer Hinweis}

Die Medizin unterliegt einem fortwährenden Entwicklungsprozess, sodass alle Angaben, insbesondere zu diagnostischen und therapeutischen Verfahren, immer nur dem Wissensstand zurzeit der Drucklegung der Leitlinie entsprechen können. Hinsichtlich der angegebenen Empfehlungen zur Therapie und der Auswahl sowie Dosierung von Medikamenten wurde die größtmögliche Sorgfalt beachtet. Gleichwohl werden die Benutzer aufgefordert, die Beipackzettel und Fachinformationen der Hersteller zur Kontrolle heranzuziehen und im Zweifelsfall einen Spezialisten zu konsultieren. Fragliche Unstimmigkeiten sollen bitte im allgemeinen Interesse der Leitlinienkoordination oder dem OL-Office mitgeteilt werden.
Der Benutzer selbst bleibt verantwortlich für jede diagnostische und therapeutische Applikation, Medikation und Dosierung.

In dieser Leitlinie sind eingetragene Warenzeichen (geschützte Warennamen) nicht besonders kenntlich gemacht. Es kann also aus dem Fehlen eines entsprechenden Hinweises nicht geschlossen werden, dass es sich um einen freien Warennamen handelt.

Das Werk ist in allen seinen Teilen urheberrechtlich geschützt. Jede Verwertung außerhalb der Bestimmung des Urhebergesetzes ist ohne schriftliche Zustimmung der OL-Redaktion unzulässig und strafbar. Kein Teil des Werkes darf in irgendeiner Form ohne schriftliche Genehmigung der OL-Redaktion reproduziert werden. Dies gilt insbesondere für Ve rvielfältigungen, Übersetzungen, Mikroverfilmungen und die Einspeicherung, Nutzung und Verwertung in elektronischen Systemen, Intranets und dem Internet.

\subsection{Ziele des Leitlinienprogramms Onkologie}

Die Arbeitsgemeinschaft der Wissenschaftlichen Medizinischen Fachgesellschaften, die Deutsche Krebsgesellschaft und die Deutsche Krebshilfe haben sich mit dem Leitlinienprogramm Onkologie $(\mathrm{OL})$ das Ziel gesetzt, gemeinsam die Entwicklung und Fortschreibung und den Einsatz wissenschaftlich begründeter und praktikabler Leitlinien in der Onkologie zu fördern und zu unterstützen. Die Basis dieses Programms beruht auf den medizinischwissenschaftlichen Erkenntnissen der Fachgesellschaften und der DKG, dem Konsens der medizinischen Fachexperten, Anwender und Patienten sowie auf dem Regelwerk für die Leitlinienerstellung der AWMF und der fachlichen Unterstützung und Finanzierung durch die Deutsche Krebshilfe. Um den aktuellen Stand des medizinischen Wissens abzubilden und den medizinischen Fortschritt zu berücksichtigen, müssen Leitlinien regelmäßig überprüft und fortgeschrieben werden. Die Anwendung des AWMFRegelwerks soll hierbei Grundlage zur Entwicklung qualitativ hochwertiger onkologischer Leitlinien sein. Da Leitlinien ein wichtiges Instrument der Qualitätssicherung und des Qualitätsmanagements in der Onkologie darstellen, sollten sie gezielt und nachhaltig in den Versorgungsalltag eingebracht werden. So sind aktive Implementierungsmaßnahmen und auch Evaluationsprogramme ein wichtiger Bestandteil der Förderung des Leitlinienprogramms Onkologie. Ziel des Programms ist es, in Deutschland professionelle und mittelfristig finanziell gesicherte Voraussetzungen für die Entwicklung und Bereitstellung hochwertiger Leitlinien zu schaffen. Denn diese hochwertigen Leitlinien dienen nicht nur dem strukturierten Wissenstransfer, sondern können auch in der Gestaltung der Strukturen des Gesundheitssystems ihren Platz finden. Zu erwähnen sind hier evidenzbasierte Leitlinien als Grundlage zum Erstellen und Aktualisieren von Disease-Management-Programmen oder die Verwendung von aus Leitlinien extrahierten Qualitätsindikatoren im Rahmen der Zertifizierung von Organtumorzentren. 


\subsection{Weitere Dokumente zu dieser Leitlinie}

Bei diesem Dokument handelt es sich um die Langversion der S3Leitlinie „Diagnostik und Therapie der Plattenepithelkarzinome und Adenokarzinome des Ösophagus“. Neben der Langversion gibt es folgende ergänzende Dokumente zu dieser Leitlinie:

- Kurzversion der Leitlinie

- Laienversion (Patientenleitlinie)

- Leitlinienreport zur Aktualsiierung der Leitlinie

- Evidenzberichte zur den Aktualsierungsrecherchen

Diese Leitlinie und alle Zusatzdokumente sind über die folgenden Seiten zugänglich.

- Leitlinienprogramm Onkologie (https://www.leitlinienprogramm-onkologie.de/leitlinien/oesophaguskarzinom/)

- AWMF (www.awmf.org)

- Deutsche Gesellschaft für Gastroenterologie, Verdauungs- und Stoffwechselkrankheiten (DGVS) (https://www.dgvs.de/ wissen-kompakt/leitlinien/leitlinien-der-dgvs/)

- Guidelines International Network (www.g-i-n.net)

\subsection{Zusammensetzung der Leitliniengruppe}

\subsubsection{Leitlinienkoordination}

Prof. Dr. med. Rainer Porschen

Chefarzt der Klinik für Innere Medizin

Klinikum Bremen

Ost Züricher Str. 40

D-28325 Bremen

Tel.: 0421/408-1221

Fax: 0421/408-2234

ainer.porschen (at) klinikum-bremen-ost.de

\section{Leitliniensekretariat/Projektmanagement}

Frau Irina John, Deutsche Gesellschaft für Gastroenterologie, Verdauungs- und Stoffwechselkrankheiten (DGVS)

E-Mail an: oesophaguskarzinom@leitlinienprogramm-onkologie.de

\subsubsection{Autoren der Leitlinie}

Porschen, R., Fischbach, W., Gockel, I., Hollerbach, S., Hölscher, A., Lynen Jansen, P., Miehlke, S., Pech, O., Stahl, M., Thuss-Patience, P., Vanhoefer, U. und die Mitarbeiter der Leitlinienkommission*

* Die Liste der Mitglieder der Leitlinienkommission sind in - Tab. 1 aufgeführt.

- Tab. 1 Beteiligte Fachgesellschaften und Organisationen.

\section{Fachgesellschaft}

Dt. Gesellschaft für Gastroenterologie, Verdauungs- und Stoffwechselkrankheiten (DGVS)

Arbeitsgemeinschaft Internistische Onkologie (AIO)

Arbeitsgemeinschaft Radiologische Onkologie (ARO)

Arbeitsgemeinschaft Supportive Maßnahmen in der Onkologie (AGSMO)

Arbeitsgemeinschaft für onkologische Rehabilitation und Sozialmedizin (AGORS)

Dt. Gesellschaft für Allgemein- und Viszeralchirurgie/Chirurgische Arbeitsgemeinschaft Onkologie (CAO-V)

Dt. Gesellschaft für Radioonkologie (DEGRO)

Dt. Gesellschaft für Ultraschall in der Medizin (DEGUM)

Dt. Gesellschaft für Allgemein- und Viszeralchirurgie (DGAV)/Chirurgische Arbeitsgemeinschaft oberer Gastrointestinaltrakt (CAOGI)

Dt. Gesellschaft für Chirurgie (DGCH)

Dt. Gesellschaft für Ernährung (DGE)

Dt. Gesellschaft für Endoskopie und bildgebende Verfahren (DGE-BV)

Dt. Gesellschaft für Ernährungsmedizin (DGEM)

Dt. Gesellschaft für Hämatologie und Medizinische Onkologie (DGHO)

Dt. Gesellschaft für Innere Medizin (DGIM)

Dt. Vereinte Gesellschaft für Klinische Chemie und Laboratoriumsmedizin (DGKL)

Dt. Gesellschaft für Nuklearmedizin (DGN)

Dt. Gesellschaft für Palliativmedizin (DGP)

Dt. Gesellschaft für Pathologie (DGP)/Bundesverband Deutscher Pathologen (BDP)

\section{Mandatsträger|} Fachexperte

Prof. Dr. Rainer Porschen (Koordinator)

Prof. Dr. Stephan Miehlke

Prof. Dr. Seufferlein

Prof. Dr. J. Trojan

Prof. Dr. Sylvie Lorenzen

Prof. Dr. Florian Lordick

PD Dr. Peter Thuß-Patience

Prof. Dr. Heinz Schmidberger

Dr. med. Maria Steingräber

Dr. Jürgen Körber

Prof. Dr. Wolfram Trudo

Knoefel

Prof. Dr. Martin Stuschke

Prof. Dr. Frederick Wenz

Prof. Dr. Josef Menzel

Prof. Dr. Ines Gockel

Prof. Dr. Dietmar Lorenz

Prof. Dr. Stefan Paul Mönig

Prof. Dr. Arnulf Hölscher

Prof. Dr. H.-J. Meyer

Prof. Dr. Ute Nöthlings

Prof. Dr. Alexander Meining Prof. Dr. Helmut Messmann

Prof. Dr. Arved Weimann

Prof. Dr. Michael Stahl

Prof. Dr. Udo Vanhoefer

PD Dr. Oliver Pech

Prof. Dr. Christoph Wagener

Prof. Dr. Matthias Schmidt

Priv. Doz. Dr.med. Philipp Lenz

Prof. Dr. Gustavo Baretton Prof. Dr. Martin Werner 
- Tab. 1 (Fortsetzung)

\begin{tabular}{|l|l|}
\hline Fachgesellschaft & $\begin{array}{l}\text { Mandatsträger/ } \\
\text { Fachexperte }\end{array}$ \\
\hline $\begin{array}{l}\text { Dt. Gesellschaft für Gastroenterologie, } \\
\text { Verdauungs-und Stoffwechselkrank- } \\
\text { heiten (DGVS)/Sektion Endoskopie }\end{array}$ & Prof. Dr. Stephan Hollerbach \\
\hline $\begin{array}{l}\text { Dt. Röntgengesellschaft (DRG) } \\
\text { Gastro Liga }\end{array}$ & Prof. Dr. Lars Grenacher \\
\hline $\begin{array}{l}\text { Arbeitsgemeinschaft für } \\
\text { Psychoonkologie (PSO) }\end{array}$ & Prof. Dr. Wolfgang Fischbach \\
\hline $\begin{array}{l}\text { Selbsthilfegruppe Speiseröhrener- } \\
\text { krankungen }\end{array}$ & $\begin{array}{l}\text { Dipl. Psychologin Dr. Ute } \\
\text { Goerling }\end{array}$ \\
\hline $\begin{array}{l}\text { Deutscher Verband für Physiotherapie } \\
\text { (ZVK) e. V. }\end{array}$ & Barbara Kade \\
\hline
\end{tabular}

\subsubsection{Beteiligte Fachgesellschaften und Organisationen}

An der Erarbeitung dieser S3-Leitlinie waren zu einzelnen Aspekten mit sozialmedizinischer Relevanz Ärztinnen und Ärzte des Kompetenz Centrums Onkologie des GKV-Spitzenverbandes und der MDK-Gemeinschaft beratend beteiligt.

Sie haben an den Abstimmungen zu den einzelnen Empfehlungen nicht teilgenommen und sind für den Inhalt dieser Leitlinie nicht verantwortlich.

\subsubsection{Arbeitsgruppen und Steuergruppe}

- Tab. 2 Steuergruppe und Arbeitsgruppen und deren Mitglieder.

\begin{tabular}{|c|c|}
\hline Gruppe & Mitglieder \\
\hline Steuergruppe & $\begin{array}{l}\text { I. Gockel, A. Hölscher, S. Hollerbach, } \\
\text { W. Fischbach, P. Lynen Jansen, S. Miehlke, } \\
\text { O. Pech, R. Porschen, M. Stahl, U. Vanhoefer }\end{array}$ \\
\hline $\begin{array}{l}\text { Arbeitsgruppe 1: } \\
\text { Risikogruppen, } \\
\text { Prävention, Screening }\end{array}$ & $\begin{array}{l}\text { W. Fischbach (AG-Leitung), S. Miehlke } \\
\text { (AG-Leitung), U. Nöthlings }\end{array}$ \\
\hline $\begin{array}{l}\text { Arbeitsgruppe 2: } \\
\text { Primärdiagnostik, } \\
\text { Diff. Diagnostik inkl. } \\
\text { Pathologie }\end{array}$ & $\begin{array}{l}\text { I. Gockel (AG-Leitung), S. Hollerbach } \\
\text { (AG-Leitung), G. Baretton, } \\
\text { L. Grenacher, A. Meining, J. Menzel, } \\
\text { J. Trojan, C. Wagener }\end{array}$ \\
\hline $\begin{array}{l}\text { Arbeitsgruppe 3: Kurativ } \\
\text { intendierte Therapie }\end{array}$ & $\begin{array}{l}\text { A. Hölscher (AG-Leitung), M. Stahl } \\
\text { (AG-Leitung), C. Ell, F. Lordick, D. Lorenz, } \\
\text { H. Messmann, H.-J. Meyer, S.P. Mönig, } \\
\text { M. Schmidt, M. Stuschke, P. Thuß-Patience, } \\
\text { R. Tholen, A. Weimann, F. Wenz, M. Werner }\end{array}$ \\
\hline $\begin{array}{l}\text { Arbeitsgruppe 4: } \\
\text { Palliation }\end{array}$ & $\begin{array}{l}\text { O. Pech (AG-Leitung), U. Vanhoefer } \\
\text { (AG-Leitung), S. Lorenzen, U. Goerling, } \\
\text { B. Kade, W.T. Knoefel, J. Körber, P. Lenz, } \\
\text { H. Schmidberger, T. Seufferlein, M. Stein- } \\
\text { gräber, T. Weihkopf }\end{array}$ \\
\hline
\end{tabular}

Tab. 2 (Fortsetzung)

\begin{tabular}{l|l} 
Gruppe & Mitglieder \\
\hline $\begin{array}{l}\text { Arbeitsgruppe } \\
\text { Qualitätsindikatoren }\end{array}$ & $\begin{array}{l}\text { R. Porschen, P. Thuß-Patience, M. Stuschke, } \\
\text { A. Hölscher, H. Messmann, O. Pech, } \\
\text { P. Lynen Jansen, B. Kade, M. Nothacker, } \\
\text { G. Barreton, M. Follmann, S. Wesselmann, } \\
\text { M. KlinkhammerSchalke }\end{array}$ \\
\hline $\begin{array}{l}\text { Arbeitsgruppe } \\
\text { Patientenleitlinie }\end{array}$ & $\begin{array}{l}\text { R. Porschen, H. Schmidberger, J. Körber, } \\
\text { S. Miehlke, I. Gockel, H. Messmann, } \\
\text { U. Vanhoefer, U. Goerling, B. Kade }\end{array}$ \\
\hline
\end{tabular}

\subsubsection{Patientenbeteiligung}

Die Leitlinie wurde unter direkter Beteiligung von einer Patientenvertreterin erstellt. Frau Barbara Kade war von Beginn in die Erstellung der Leitlinie eingebunden und nahm mit eigenem Stimmrecht an den Konsensuskonferenzen teil.

\subsubsection{Methodische Begleitung}

durch das Leitlinienprogramm Onkologie:

- Dr. Markus Follmann MPH MSc (OL-Office), Berlin

- Dipl. Soz.Wiss. Thomas Langer (OL-Office), Berlin

- Dr. med. Monika Nothacker, MPH (AWMF-IMWi), Berlin

durch die federführende Fachgesellschaft DGVS:

- Priv. Doz. Dr. Petra Lynen Jansen (DGVS), Berlin

Durch externe Auftragnehmer

- Dr. Paul Freudenberger (CGS User Group Leitlinienportal)

- Erik Wolfarth (CGS User Group Leitlinienportal)

- Dr. med. Simone Wesselmann, MBA (Aktualisierung der Qualitätsindikatoren)

\subsection{Verwendete Abkürzungen}

\begin{tabular}{|l|l|}
\hline Abkürzung & Erläuterung \\
\hline Abd. & Abdominal \\
\hline AC & Adenokarzinom \\
\hline AEG-Tumoren & Karzinome des gastroösophagealen Übergangs \\
\hline AG & Arbeitsgruppe \\
\hline Al & Autofluorescence Imaging \\
\hline APC & Argon Plasma Coagulation \\
\hline ASS & Acetylsalicylsäure \\
\hline AWMF & Arbeitsgemeinschaft der Wissenschaftlichen \\
\hline AWMF-IMWi & Medizinischen Fachgesellschaften \\
\hline BMI & Körpermasseindex \\
\hline BÖ & Barrett Ösophagus \\
\hline CEUS & Kontrastverstärkte Sonografie \\
\hline Cerv. & cervical \\
\hline CGS & Clinical Guideline Services \\
\hline
\end{tabular}




\begin{tabular}{|c|c|}
\hline Abkürzung & Erläuterung \\
\hline $\mathrm{Cl}$ & Konfidenzintervall \\
\hline CR & Komplette Remission \\
\hline CRT & Chemoradiotherapie \\
\hline СT & Computertomografie \\
\hline CTV & Klinisches Zielvolumen \\
\hline DELBI & Deutsches Leitlinienbewertungsinstrument \\
\hline DN & de novo \\
\hline DKG & Deutsche Krebsgesellschaft \\
\hline EBUS & Endobronchialer Ultraschall \\
\hline EK & Expertenkonsens \\
\hline EMEA & European Medicine Agency \\
\hline EMR & Endoskopische Mukosaresektion \\
\hline EKG & Elektrokardiogramm \\
\hline ER & endoskopische Resektion \\
\hline ERAS & Enhanced Recovery after Surgery (ERAS) \\
\hline ESD & Endoskopische Submukosa-Dissektion \\
\hline EUS & Endoskopischer Ultraschall \\
\hline FDG-PET & 18F-Fluordeoxyglukose-Positronenemissionstomografie \\
\hline FICE & Fujinon intelligent chromoendoscopy \\
\hline FISH & Fluoreszenz-in-situ-Hybridisierung \\
\hline FKJ & Feinnadelkatheter-Jejunostomie \\
\hline FNP & Feinnadel-Biopsie \\
\hline GCP & Good Clinical Practice \\
\hline GIN & Guideline International Network \\
\hline GIST & Gastrointestinaler Stromatumor \\
\hline Gy & Gray \\
\hline HDTV & high-resolution endoscopy \\
\hline HER2 & human epidermal growth factor receptor 2 \\
\hline HGD & Hochgradige Dysplasie \\
\hline HGIEN & Hochgradige intraepitheliale Neoplasie \\
\hline HNO & Hals-Nasen-Ohrenheilkunde \\
\hline HR & Hazard Ratio \\
\hline HTA & Health Technology Assessment \\
\hline IEN & intraepitheliale Neoplasie \\
\hline $\mathrm{IHC}$ & Immunhistochemie \\
\hline IQWIG & $\begin{array}{l}\text { Institut für Qualität und Wirtschaftlichkeit im } \\
\text { Gesundheitswesen }\end{array}$ \\
\hline LA & Leitlinienadaptation \\
\hline LGD & Niedriggradige Dysplasie \\
\hline LGIEN & Niedriggradige intraepitheliale Neoplasie \\
\hline LITT & Laser-induzierte Thermotherapie \\
\hline LoE & Level of Evidence \\
\hline Lugol-CE & Lugol'sche Lösung \\
\hline MDCT & Multi-detector Computed Tomography \\
\hline MDK & Medizinischer Dienst der Krankenversicherung \\
\hline MIC & Minimal invasive Chirurgie \\
\hline
\end{tabular}

\begin{tabular}{|c|c|}
\hline Abkürzung & Erläuterung \\
\hline MRT & Magnetresonanztomografie \\
\hline NBI & Narrow-band Imaging \\
\hline NCCN & National Comprehensive Cancer Network \\
\hline NICE & National Institute of Clinical Excellence \\
\hline NGC & National Guideline Clearinghouse \\
\hline NHMRC & National Health and Medical Research Council \\
\hline NHS & National Health Service \\
\hline NRS & Nutritional Risiko Score \\
\hline NSAR & Nichtsteroidale Antirheumatika \\
\hline NZGG & New Zealand Guidelines Group \\
\hline $\mathrm{OL}$ & Leitlinienprogramm Onkologie \\
\hline OP & Operation \\
\hline OPS & Operationen- und Prozedurenschlüssel \\
\hline OR & Odds Ratio \\
\hline ÖGD & Ösophagogastroduodenoskopie \\
\hline ÖG] & Ösophagogastrale Junktion \\
\hline ÖGÜ & Ösophagogastraler Übergang \\
\hline $\mathrm{PET} / \mathrm{CT}$ & Positronen-Emissions-Tomografie/Computertomografie \\
\hline PEG & Perkutane endoskopische Gastrostomie \\
\hline PDT & Photodynamische Therapie \\
\hline PICO & Population, Intervention, Comparison, Outcome \\
\hline 4-QPE's & 4-Quadranten Probeexzisionen \\
\hline QI & Qualitätsindikatoren \\
\hline QoL & Quality of Life \\
\hline RCT & Randomisierte klinische Studien \\
\hline RFA & Radiofrequenzablation \\
\hline ROC & Receiver Operating Characteristic \\
\hline RÖ & Röntgen \\
\hline RR & Relatives Risiko \\
\hline RT & Radiotherapie \\
\hline SCC & squamous cell carcinoma \\
\hline SEMS & Selbstexpandierende Metallgitterstents \\
\hline SGB & Sozialgesetzbuch \\
\hline SIGN & Scottish Intercollegiate Guidelines Network \\
\hline SIRT & Selektive interne Radiotherapie \\
\hline SOP & Transparente klinische Ablaufstandards \\
\hline SR & Systematischer Review \\
\hline SIR & Standardisierte Inzidenzrate \\
\hline TACE & Transarterielle Chemoembolisation \\
\hline TNM & TNM-Klassifikation \\
\hline TRG & Tumorregressionsgrad \\
\hline UICC & $\begin{array}{l}\text { UICC (Union internationale contre le cancer)-Klassifi- } \\
\text { kation }\end{array}$ \\
\hline US & Ultraschall \\
\hline WLE & Weißlicht Endoskopie \\
\hline WHO & World Health Organisation \\
\hline
\end{tabular}




\section{Einführung}

\subsection{Geltungsbereich und Zweck}

\subsubsection{Zielsetzung und Fragestellung}

Beim Speiseröhrenkrebs (Ösophaguskarzinom) werden zwei verschiedene Gewebetypen, das Adenokarzinom und das Plattenepithelkarzinom unterschieden. 2018 sollen nach der Prognose des Robert-Koch-Instituts in Deutschland 5700 Männer und 1700 Frauen neu an Speiseröhrenkrebs erkranken - also eine steigende Tendenz. Dies entspricht einem Anteil von 3\% bei Männern und $1 \%$ bei Frauen an allen bösartigen Neubildungen. Das Ösophaguskarzinom zählt zu den Tumorarten mit einer sehr schlechten Prognose: die relative 10-Jahres Überlebensrate liegt bei $16-17 \%$ [1].

Die Diagnostik und Therapie des Ösophaguskarzinoms stellt mehr noch als bei anderen Tumorentitäten hohe Anforderungen an die beteiligten Fachdisziplinen. Dies ist auf der einen Seite durch die enge Nachbarschaft der Speiseröhre zum Bronchialsystem und zur Lunge bedingt - eine Tatsache, die erhebliche technische Anforderungen an das operative und therapeutische Vorgehen stellt. Deshalb ist ein hoher Grad an Interdisziplinarität erforderlich, um die Patienten nach subtiler Diagnostik einer stadiengerechten Therapie zuzuführen. Dies beinhaltet besonders die Entscheidung, welche Patienten alleine durch eine Operation, welche Patienten durch eine Kombination einer neoadjuvanten präoperativen Radiochemotherapie plus Operation oder welche sogar durch eine alleinige Radiochemotherapie behandelt werden sollen. Zudem sind neue diagnostische Verfahren (z. B. PET-CT) in die Stufendiagnostik des Ösophaguskarzinoms eingeführt worden, deren Stellenwert noch nicht eindeutig definiert und festgelegt worden ist.

Auf der anderen Seite ist die Therapie des Ösophaguskarzinoms - besonders bei den Plattenepithelkarzinomen - durch die Tatsache erschwert, dass durch den häufig begleitenden Alkoholund Tabakkonsum Begleiterkrankungen vorliegen, die die Möglichkeiten einer einzuschlagenden Therapie erheblich beeinflussen.

Mit der Publikation 2015 wurde erstmal eine aktuelle, alle Themen abdeckende Leitlinie zum Thema „Speiseröhrenkrebs“ erstellt [2], die eine Standardisierung in der Prävention, Diagnostik, Therapie, Palliation und Nachsorge ermöglichte und somit das Ziel verfolgte, die Behandlungsergebnisse zu verbessern. Die vorliegende Aktualisierung der Leitlinie beabsichtigt, diese Aktualität zu erhalten und gleichzeitig ein schnelles Reagieren auf wesentliche Änderungen zu ermöglichen. Unter dem Aspekt der „living guideline" wird somit eine kontinuierliche, standardisierte Aktualisierung vorgenommen. Die Inhalte der Leitlinie werden dabei jährlich auf Basis aktueller Studiendaten und neuer Publikationen, Umfragen zu Qualität und Inhalten der Leitlinie und Rückmeldungen aus der Leitliniengruppe geprüft und gegebenenfalls aktualisiert.

\subsubsection{Adressaten}

In der Leitlinie „Ösophaguskarzinom“ wird das gesamte Spektrum der Prävention, Diagnostik und Therapie des Ösophaguskarzinoms behandelt. Sie wendet sich somit an alle Ärzte und Berufs- gruppen, die Patienten mit Ösophaguskarzinom und/oder Risikofaktoren für ein Ösophaguskarzinom behandeln. Hierzu zählen Fachärzte für Innere Medizin, Gastroenterologie, Hämatologie und Onkologie, Chirurgie, Radiologie, Strahlentherapie, Pathologie, Nuklearmedizin, Palliativmedizin sowie onkologisch tätige Fachkräfte und Berufsgruppen, die an der Versorgung von Patienten mit Ösophaguskarzinom beteiligt sind.

Weitere Adressaten der Leitlinie sind Organisationen der Patientenberatung, Selbsthilfegruppen sowie Entscheidungsund Kostenträger im Gesundheitswesen.

Der Ansatz der Leitlinie ist interdisziplinär und sektorenübergreifend, da sowohl stationäre/teilstationäre als auch ambulante Versorgungsstrukturen eingeschlossen werden.

\subsubsection{Gültigkeitsdauer und Aktualisierungsverfahren}

Die S3-Leitlinie in der Version 2 ist bis zur nächsten Aktualisierung gültig, maximal aber 5 Jahre. Es ist vorgesehen, die Inhalte der Leitlinie im Rahmen eines sogenannten „living-guidline-Ansatzes“ jährlich auf Basis aktueller Studiendaten und neuer Publikationen sowie Rückmeldungen aus der Leitliniengruppe zu prüfen und gegebenenfalls zu aktualisieren. Hierzu wird einmal jährlich eine systematische Literaturrecherche und Evidenzbeurteilung durchgeführt.

Kommentare und Hinweise für den Aktualisierungsprozess sind ausdrücklich erwünscht und können an: oesophaguskarzinom@leitlinienprogramm-onkologie.de adressiert werden.

\subsection{Grundlagen der Methodik}

Die methodische Vorgehensweise bei der Erstellung der Leitlinie ist im Leitlinienreport dargelegt. Dieser ist im Internet z. B. auf den Seiten des Leitlinienprogramms Onkologie (https://www.leitlinienprogramm-onkologie.de/leitlinien/oesophaguskarzinom/) und den Seiten der AWMF (http://www.awmf.org/) frei verfügbar.

\subsubsection{Schema der Evidenzgraduierung nach Oxford}

(Version 2009)

Zur Klassifikation des Verzerrungsrisikos der identifizierten Studien wurde in dieser Leitlinie das in $>$ Tab. 3 aufgeführte System des Oxford Centre for Evidence-based Medicine in der Version von 2009 verwendet. Dieses System sieht die Klassifikation der Studien für verschiedene klinische Fragestellungen (Nutzen von Therapie, prognostische Aussagekraft, diagnostische Wertigkeit) vor.

\subsubsection{Schema der Empfehlungsgraduierung}

Die Methodik des Leitlinienprogramms Onkologie sieht eine Vergabe von Empfehlungsgraden durch die Leitlinienautoren im Rahmen eines formalen Konsensusverfahrens vor. Dementsprechend wurden durch die AWMF moderierte, nominale Gruppenprozesse bzw. strukturierte Konsensuskonferenzen oder DELPHIAbstimmungen durchgeführt. Im Rahmen dieser Prozesse wurden die Empfehlungen von den stimmberechtigten Mandatsträgern (siehe Kapitel 1.9.3.) formal abgestimmt. Die Ergebnisse der jeweiligen Abstimmungen (Konsensstärke) sind entsprechend den Kategorien in $>$ Tab. 5 den Empfehlungen zugeordnet. 
- Tab. 3 Schema der Evidenzgraduierung nach Oxford (Version März 2009).

\begin{tabular}{|c|c|c|c|c|}
\hline Level & $\begin{array}{l}\text { Therapy/Prevention, } \\
\text { Aetiology/Harm }\end{array}$ & Prognosis & Diagnosis & $\begin{array}{l}\text { Differential diagnosis/ } \\
\text { symptom prevalence study }\end{array}$ \\
\hline $1 \mathrm{a}$ & SR (with homogeneity) of RCTs & $\begin{array}{l}\text { SR (with homogeneity) inception } \\
\text { cohort studies; CDR validated in } \\
\text { different populations }\end{array}$ & $\begin{array}{l}\text { SR (with homogeneity) of Level } 1 \\
\text { diagnostic studies; CDR with 1b } \\
\text { studies from different clinical } \\
\text { centers }\end{array}$ & $\begin{array}{l}\text { SR (with homogeneity) of } \\
\text { prospective cohort studies }\end{array}$ \\
\hline $1 b$ & $\begin{array}{l}\text { Individual RCT (with narrow } \\
\text { Confidence Interval) }\end{array}$ & $\begin{array}{l}\text { Individual inception cohort study } \\
\text { with }>80 \% \text { follow-up; CDR vali- } \\
\text { dated in a single population }\end{array}$ & $\begin{array}{l}\text { Validating cohort study with } \\
\text { good reference standards; or } \\
\text { CDR tested within one clinical } \\
\text { centre }\end{array}$ & $\begin{array}{l}\text { Prospective cohort study with } \\
\text { good follow-up }\end{array}$ \\
\hline $2 a$ & $\begin{array}{l}\text { SR (with homogeneity) of } \\
\text { cohort studies }\end{array}$ & $\begin{array}{l}\text { SR (with homogeneity) of either } \\
\text { retrospective cohort studies or } \\
\text { untreated control groups in RCTs }\end{array}$ & $\begin{array}{l}\text { SR (with homogeneity) of Level } \\
>2 \text { diagnostic studies }\end{array}$ & $\begin{array}{l}\text { SR (with homogeneity) of Level } \\
2 \mathrm{~b} \text { and better studies }\end{array}$ \\
\hline $2 b$ & $\begin{array}{l}\text { Individual cohort study } \\
\text { (including low quality RCT; } \\
\text { e. g., }<80 \% \text { follow-up) }\end{array}$ & $\begin{array}{l}\text { Retrospective cohort study or } \\
\text { follow-up of untreated control } \\
\text { patients in an RCT; Derivation of } \\
\text { CDR or validated on split-sample } \\
\text { only }\end{array}$ & $\begin{array}{l}\text { Exploratory cohort study with } \\
\text { good reference standards; CDR } \\
\text { after derivation, or validated } \\
\text { only on splitsample or databases }\end{array}$ & $\begin{array}{l}\text { Retrospective cohort study, or } \\
\text { poor follow-up }\end{array}$ \\
\hline $2 c$ & $\begin{array}{l}\text { “Outcomes” Research; } \\
\text { Ecological studies }\end{array}$ & “Outcomes” Research & & Ecological studies \\
\hline $3 a$ & $\begin{array}{l}\text { SR (with homogeneity) of } \\
\text { case-control studies }\end{array}$ & & $\begin{array}{l}\text { SR (with homogeneity) of } 3 \mathrm{~b} \text { and } \\
\text { better studies }\end{array}$ & $\begin{array}{l}\text { SR (with homogeneity) of } 3 \mathrm{~b} \text { and } \\
\text { better studies }\end{array}$ \\
\hline $3 b$ & Individual Case-Control Study & & $\begin{array}{l}\text { Non-consecutive study; or } \\
\text { without consistently applied } \\
\text { reference standards }\end{array}$ & $\begin{array}{l}\text { Non-consecutive cohort study; } \\
\text { or very limited population }\end{array}$ \\
\hline 4 & $\begin{array}{l}\text { Case-series (and poor quality } \\
\text { cohort and case-control } \\
\text { studies) }\end{array}$ & $\begin{array}{l}\text { Case-series (and poor quality } \\
\text { prognostic cohort studies) }\end{array}$ & $\begin{array}{l}\text { Case-control study, poor or non- } \\
\text { independent reference standard }\end{array}$ & $\begin{array}{l}\text { Case-series or superseded } \\
\text { reference standards }\end{array}$ \\
\hline 5 & $\begin{array}{l}\text { Expert opinion without } \\
\text { explicit critical appraisal, or } \\
\text { based on physiology, bench } \\
\text { research or "first principles" }\end{array}$ & $\begin{array}{l}\text { Expert opinion without explicit } \\
\text { critical appraisal, or based on } \\
\text { physiology, bench research or } \\
\text { "first principles" }\end{array}$ & $\begin{array}{l}\text { Expert opinion without explicit } \\
\text { critical appraisal, or based on } \\
\text { physiology, bench research or } \\
\text { "first principles" }\end{array}$ & $\begin{array}{l}\text { Expert opinion without explicit } \\
\text { critical appraisal, or based on } \\
\text { physiology, bench research or } \\
\text { "first principles" }\end{array}$ \\
\hline
\end{tabular}

In der Leitlinie werden zu allen evidenzbasierten Statements und Empfehlungen das Evidenzlevel der zugrunde liegenden Studien sowie bei Empfehlungen zusätzlich die Stärke der Empfehlung (Empfehlungsgrad) ausgewiesen. Hinsichtlich der Stärke der Empfehlung werden in dieser Leitlinie drei Empfehlungsgrade unterschieden ( $\vee$ Tab. 4 ), die sich auch in der Formulierung der Empfehlungen jeweils widerspiegeln.

Die Entscheidungskriterien für die Festlegung der Empfehlungsgrade werden im Leitlinienreport zu dieser Leitlinie erläutert.

\subsubsection{Statements}

Als Statements werden Darlegungen oder Erläuterungen von spezifischen Sachverhalten oder Fragestellungen ohne unmittelbare Handlungsaufforderung bezeichnet. Sie werden entsprechend der Vorgehensweise bei den Empfehlungen im Rahmen eines formalen Konsensusverfahrens verabschiedet und können entweder auf Studienergebnissen oder auf Expertenmeinungen (Expertenkonsens) beruhen.
- Tab. 4 Schema der Empfehlungsgraduierung.

\begin{tabular}{|l|l|l|}
\hline $\begin{array}{l}\text { Empfehlungs- } \\
\text { grad }\end{array}$ & Beschreibung & Ausdrucksweise \\
\hline A & Starke Empfehlung & soll/soll nicht \\
\hline B & Empfehlung & sollte/sollte nicht \\
\hline 0 & Empfehlung offen & $\begin{array}{l}\text { kann/kann } \\
\text { verzichtet werden }\end{array}$ \\
\hline
\end{tabular}

\subsubsection{Expertenkonsens (EK)}

Statements/Empfehlungen, für die eine Bearbeitung auf der Grundlage von Expertenkonsens der Leitliniengruppe beschlossen wurde, sind als „Expertenkonsens“ ausgewiesen. Für die Graduierung des Expertenkonsenes wurden keine Symbole bzw. Buchstaben verwendet, die Empfehlungsstärke bei (Experten)konsensbasierten Empfehlungen ergibt sich aus der verwendeten Formulierung (soll/sollte/kann) entsprechend der Abstufung in

- Tab. 4. 
- Tab. 5 Konsensusstärke.

\begin{tabular}{|l|l|}
\hline Konsenstärke & Prozentuale Zustimmung \\
\hline Starker Konsens & $>95 \%$ der Stimmberechtigten \\
\hline Konsens & $>75-95 \%$ der Stimmberechtigten \\
\hline Mehrheitliche Zustimmung & $>50-75 \%$ der Stimmberechtigten \\
\hline Dissens & $<50 \%$ der Stimmberechtigten \\
\hline
\end{tabular}

\subsubsection{Unabhängigkeit und Darlegung möglicher Interessenkonflikte}

Die Deutsche Krebshilfe stellte über das Leitlinienprogramm Onkologie (OL) finanzielle Mittel für insgesamt drei Jahre zur Verfügung (2017/18 - 2020). Für die Aktualisierung 2018 wurden die Mittel eingesetzt für Büromaterial, Literaturbeschaffung/ Evidenzbeurteilungen, Delphi-Befragung und die Treffen der Steuergruppe (Technik, Verpflegung, Reisekosten der Teilnehmer). Die Erarbeitung der Leitlinie erfolgte in redaktioneller Unabhängigkeit von der finanzierenden Organisation. Die Leitliniengruppenteilnehmer arbeiteten ehrenamtlich.

Die Leitliniengruppenmitglieder legten während des Leitlinienprozesses vor der formalen Abstimmung der Empfehlungen eine schriftliche Erklärung zu Interessen anhand des AWMF-Formblattes Stand 08.10.2010 vor. Die offengelegten Interessen sind in einer standardisierten Tabelle im Leitlinienreport auf den Webseiten des Leitlinienprogramms Onkologie (https://www.leitlinienprogramm-onkologie.de/leitlinien/oesopha-guskarzinom/) und der AWMF (https://www.awmf.org/leitlinien/detail/I/021-023OL. html) einsehbar. Verantwortlich für die Aktualisierung war zusammen mit dem Koordinator eine interdisziplinär zusammengesetzte Steuergruppe ( $\bullet$ Tab.2). In dieser wurden alle Schritte der Aktualisierung besprochen. Bei einem Treffen der Steuergruppe am 05.07.2017 wurde nach interner Diskussion folgendes Procedere zur Bewertung von und zum Umgang mit den offengelegten Interessen festgelegt:

Bei positiven Angaben in den Rubriken: Berater- bzw. Gutachtertätigkeit oder bezahlte Mitarbeit in einem wissenschaftlichen Beirat eines Unternehmens der Gesundheitswirtschaft (z. B. Arzneimittelindustrie, Medizinproduktindustrie), eines kommerziell orientierten Auftragsinstituts oder einer Versicherung oder Besitz von Geschäftsanteilen, Aktien, Fonds mit Beteiligung von Unternehmen der Gesundheitswirtschaft oder Persönliche Beziehungen zu einem Vertretungsberechtigten eines Unternehmens erfolgte eine Bewertung in gering, moderat und hoch. Angaben der Rubriken Vortrags- und Schulungstätigkeit bzw. Wissenschaftlicher Schwerpunkt/Studien wurden in dieser Gruppe grundsätz- lich als gering bewertet. Bei Vorliegen eines geringen Interessenkonflikts wurden aufgrund der vorhandenen protektiven Faktoren (unabhängige Evidenzsuche und Bewertung, formales Konsensverfahren, Einbeziehen aller relevanten Stakeholder) keine weitere Maßnahme als erforderlich erachtet. Bei Vorliegen eines moderaten Interessenkonflikts erfolgte eine Abstimmungsenthaltung und Doppelabstimmung, um festzustellen, ob die Enthaltung zu einer geänderten Zustimmungsquote führen würde. Wenn aufgrund der offengelegten Sachverhalte in diesen Kategorien ein hohes Risiko für einen Interessenkonflikt angenommen werden musste, sollte diese Personen nicht an der Abstimmung und der Diskussion der entsprechenden Empfehlungen teilnehmen.

Die Beurteilung wurde durch eine Kommission der Leitliniengruppe aus drei Klinikern (Hölscher (DGCH), Hollerbach (DGVS), Vanhoefer (DGHO)) und zwei Methodikern (Langer (OL-Office), Nothacker (AWMF)) vorgenommen. Die Beurteilung 2018 erfolgte für die in 2018 bearbeiteten Themen. Nach Sichtung der eingegangenen Rückmeldungen der Fachexperten - und wenn erforderlich - persönlicher Rücksprache wurde für die Aspekte "computergestützte digitale Filter" für die Chromoendoskopie bei Autoren aufgrund von Tätigkeit in Advisory Boards von Endoskopiefirmen für dieses Thema ein moderater Interessenkonflikt gesehen und eine Doppelabstimmung avisiert, eine solche Tätigkeit lag jedoch nicht vor. Weiterhin wurde zusätzlich zu den oben genannten Konstellationen für die Empfehlung zur Referenzpathologie ein moderater Interessenkonflikt bei den Vertretern des Berufsverbandes der Pathologen gesehen, hier erfolgte eine Doppelabstimmung. Es ergab sich keine Änderung des Konsenses.

An dieser Stelle möchten wir allen Experten und Mitarbeitern für ihre ausschließlich ehrenamtliche Mitarbeit an dem Projekt danken.

\section{Patienteninformation und Aufklärung}

\subsection{Informationsmaterial}

\begin{tabular}{|l|l|l|}
\hline 3.1. & Konsensbasierte Empfehlung & $\begin{array}{l}\text { geprüft } \\
\mathbf{2 0 1 8}\end{array}$ \\
\hline EK & $\begin{array}{l}\text { Informationsmaterialien (Print- und Internetmedien) sollen } \\
\text { nach definierten Qualitätskriterien für Gesundheitsinforma- } \\
\text { tionen erstellt und den Patienten zur Verfügung gestellt } \\
\text { werden, um sie durch eine verständliche Risikokommunika- } \\
\text { tion (z. B. Angabe von absoluten Risikoreduktionen) in ihrer } \\
\text { selbstbestimmten Entscheidung für oder gegen die medizi- } \\
\text { nischen Maßnahmen zu unterstützen. }\end{array}$ \\
\hline $\begin{array}{l}\text { Konsen- } \\
\text { sstärke }\end{array}$ & \begin{tabular}{l} 
Starker Konsens (100\%) \\
\hline
\end{tabular} \\
\hline
\end{tabular}




\subsection{Grundprinzipien einer patientenzentrierten Kommunikation}

\begin{tabular}{|c|c|c|}
\hline 3.2. & Evidenzbasierte Empfehlung & $\begin{array}{l}\text { geprüft } \\
2018\end{array}$ \\
\hline $\begin{array}{l}\text { Empfeh- } \\
\text { lungs- } \\
\text { grad } \\
\text { A }\end{array}$ & \multicolumn{2}{|c|}{$\begin{array}{l}\text { Die Art der Vermittlung von Informationen und der Auf- } \\
\text { klärung der Patientin soll nach denfolgenden Grundprinzi- } \\
\text { pien einer patientenzentrierten Kommunikation, die eine } \\
\text { partizipative Entscheidungsfindung ermöglicht, erfolgen: } \\
\text { - Ausdruck von Empathie und aktives Zuhören, } \\
\text { - direktes und einfühlsames Ansprechen schwieriger } \\
\text { Themen, } \\
\text { - wenn möglich, Vermeidung von medizinischem } \\
\text { Fachvokabular, ggf. Erklärung von Fachbegriffen, } \\
\text { - Strategien, um das Verständnis zu verbessern (Wieder- } \\
\text { holung, Zusammenfassung wichtiger Informationen, } \\
\text { Nutzung von Grafiken u. a.) } \\
\text { - Ermutigung, Fragen zu stellen } \\
\text { - Erlaubnis und Ermutigung, Gefühle auszudrücken } \\
\text { - weiterführende Hilfe anbieten (siehe Abschnitt } \\
\text { Psychoonkologie) }\end{array}$} \\
\hline $\begin{array}{l}\text { Level of } \\
\text { Evidence } \\
\text { 1b }\end{array}$ & \multicolumn{2}{|c|}{$\begin{array}{l}\text { Leitlinienadaptation: S3-Leitlinie Mammakarzinom Juli } \\
2012 \text { [3] }\end{array}$} \\
\hline $\begin{array}{l}\text { Konsens- } \\
\text { stärke }\end{array}$ & \multicolumn{2}{|l|}{ Starker Konsens (100\%) } \\
\hline
\end{tabular}

\subsection{Therapieaufklärungsgespräch}

\begin{tabular}{|c|c|c|}
\hline 3.3. & Konsensbasierte Empfehlung & $\begin{array}{l}\text { geprüft } \\
2018\end{array}$ \\
\hline EK & \multicolumn{2}{|c|}{$\begin{array}{l}\text { Als Inhalte eines Therapieaufklärungsgespräches sollten je } \\
\text { nach Therapieansatz folgende Punkte angesprochen werden: } \\
\text { Kurative Therapie } \\
\text { - Endoskopische Therapie bei Frühkarzinomen } \\
\text { - Neoadjuvante Strategie-Prinzipien, Ziele } \\
\text { - Radiochemotherapie: Dauer und Durchführung, } \\
\text { Nebenwirkungen, Spätfolgen } \\
\text { - Operative Therapie: Zweihöhleneingriff, Technik und } \\
\text { Rekonstruktionsverfahren: Thorakale/kollare Anastomose } \\
\text { - Risiken } \\
\text { - Ernährungstherapie und -sonde, Feinnadelkatheter- } \\
\text { jejunostomie (FKJ) } \\
\text { - Funktionelle Auswirkungen } \\
\text { - Rehabilitation } \\
\text { Palliative Therapie } \\
\text { - Radio(chemo)therapie } \\
\text { - Chemotherapie } \\
\text { - Endoskopische Therapie (u. a. Stentimplantation) } \\
\text { - Perkutane endoskopische Gastrostomie (PEG) } \\
\text { - Palliativmedizin inkl. Schmerztherapie } \\
\text { Betont werden sollten auch Notwendigkeit und Möglich- } \\
\text { keiten der Patientenkooperation } \\
\text { - Compliance bzw. Adhärenz } \\
\text { - Konditionierung } \\
\text { - Mobilisierung/körperlicher Aktivität } \\
\text { - Ernährung } \\
\text { - Rehabilitation }\end{array}$} \\
\hline
\end{tabular}

\begin{tabular}{|l|l|}
\hline 3.3. & $\begin{array}{l}\text { geprüft } \\
2018\end{array}$ \\
\hline & $\begin{array}{l}\text { Konsensbasierte Empfehlung } \\
\text { - Nachsorge }\end{array}$ \\
\hline $\begin{array}{l}\text { Angeboten werden sollte in jedem Fall eine psychoonkolo- } \\
\text { gische Unterstützung. }\end{array}$ \\
\hline $\begin{array}{l}\text { Konsens- } \\
\text { stärke }\end{array}$ & Konsens (76\%) \\
\hline
\end{tabular}

\section{Hintergrund}

Die Empfehlungen zu Patienteninformation und Aufklärung basieren auf entsprechenden Empfehlungen aus der S3-Leitlinie zum Mammakarzinom [3].

Gerade für Krebserkrankungen ist die Bedeutung der Arzt-Patienten-Beziehung für den Krankheitsverlauf und das Erreichen des Therapieziels unbestritten. Im heutigen Verständnis ist der Arzt vor allem Partner des Patienten mit besonderem Fachwissen und Können.

Information und Beratung müssen ergebnisoffen sein. Die vom Arzt medizinethisch zu berücksichtigenden Prinzipien sind die Fürsorge und das Nicht-Schaden unter Anerkennung der Selbstbestimmung und Autonomie des Patienten in der Phase einer existenziellen Bedrohung. Der Patientenautonomie kommt dabei höchste Priorität zu. Entscheidungen für oder gegen eine Therapie sind für das ärztliche Handeln absolut bindend. Ein zeitlicher Druck zur Entscheidungsfindung ist zu vermeiden [4, 5].

Dem Patienten muss mit Empathie das vertrauensvolle „gut Aufgehobensein“ in der Betreuung eines interdisziplinären und multiprofessionellen Teams mit verschiedenen klar definierten Kompetenzen und Aufgaben einschließlich Ernährungs- und Physiotherapie, Psychoonkologie und Sozialdienst vermittelt werden. Dies wird häufig mehrere Aufklärungsgespräche mit unterschiedlichen Partnern des Teams erforderlich machen. Für den Patienten und seine Angehörigen müssen dabei Koordination und Verantwortlichkeit klar erkennbar sein. Die einzelnen Abläufe sollten unter Einbeziehung von Informations- und Aufklärungsgespräch in einer Standard Operative Procedure festgelegt sein.

Patienteninformation und -aufklärung über die Karzinomdiagnose und das Therapiekonzept sollten sorgfältig und detailliert, möglichst stufenweise erfolgen. Dem Informationsbedürfnis des Patienten ist umfassend Rechnung zu tragen [6]. Durch das Internet haben viele Patienten bereits frühzeitig Informationen über ihre Krebserkrankung erhalten, die im Arzt-Patienten Gespräch thematisiert werden müssen und der Kommentierung bedürfen.

Grundlage der Beratung sollten die auf den Leitlinien fußenden Empfehlungen der jeweiligen Onkologischen Konferenz (Tumorboard) sein, die auf den Patienten individuell abgestimmt werden müssen. Diese betreffen mögliche Einschränkungen innerhalb des Therapiekonzepts z. B. durch die Komorbidität des Patienten, aber auch dessen Präferenzen und Wünsche zur Lebensplanung.

Aufgrund der multimodalen Therapiekonzepte für das Ösophaguskarzinom kann die Aufklärung in einem vertrauensbildenden gemeinsamen Gespräch z. B. durch Chirurgen und (Radio-)Onkologen sinnvoll sein - gerade im neoadjuvanten Vorgehen oder bei 


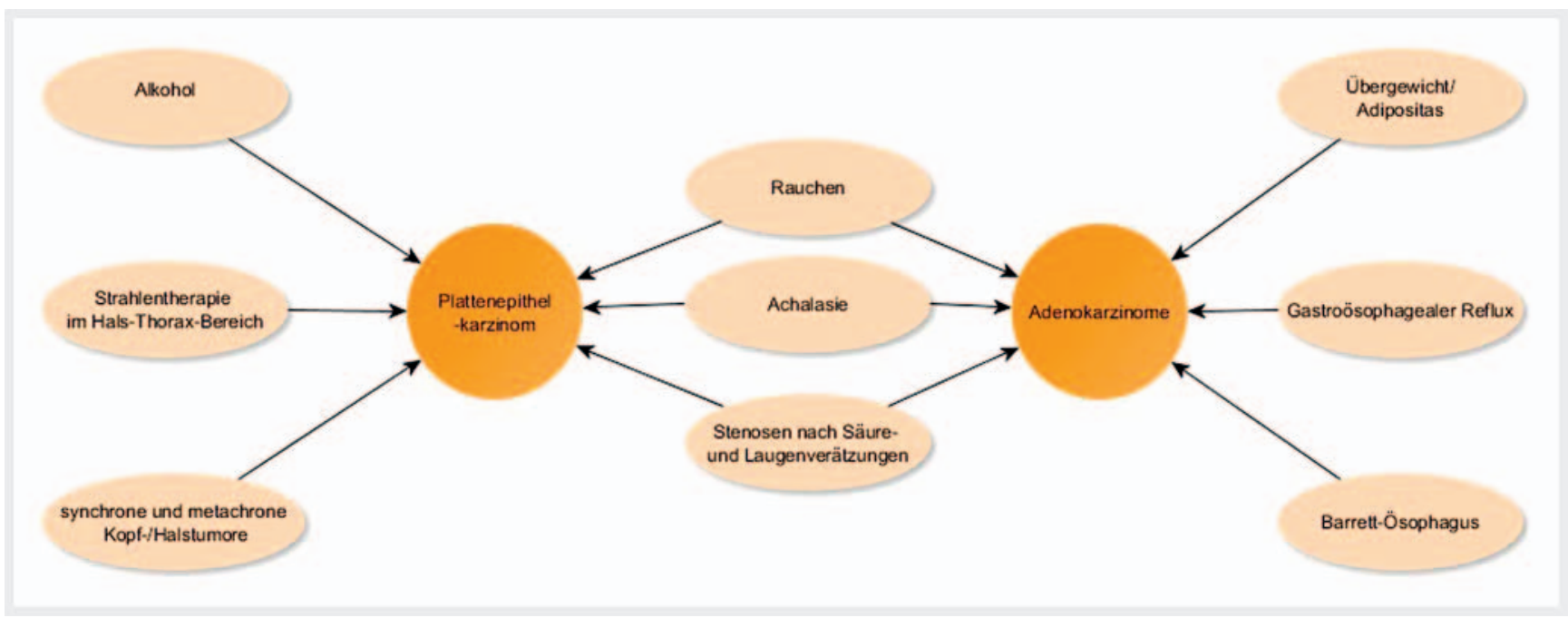

- Abb. 1 Bekannte Risikofaktoren für die Entstehung eines Ösophaguskarzinoms. [rerif]

Abwägung der Alternative zwischen Operation und definitiver Radio-Chemotherapie. Deutlich müssen Risiken und Nebenwirkungen, Toxizität, Spätfolgen und auch die Auswirkungen auf die Lebensführung (Ernährung) mit der zu erwartenden Lebensqualität des Patienten besprochen werden. Thematisiert werden sollten Erwartungen und Ängste des Patienten sowie Notwendigkeit und Möglichkeiten der Kooperation. Falls in Betracht kommend, ist die Teilnahme an einer Therapiestudie mit dem Patienten abzuwägen. Ggf. muss dabei sorgfältig das Prinzip der Randomisierung erläutert werden.

Das Arzt-Patienten-Gespräch sollte mit Empathie patientenzentriert in ruhiger Atmosphäre durchgeführt werden. Wenn es dem Wunsch des Patienten entspricht, sind Partner und nächste Angehörige einzubeziehen. Die Beratung muss in einer dem Patienten verständlichen Sprache erfolgen. Sowohl die Verharmlosung der Erkrankung und des Operationsrisikos als auch eine paralysierende Verängstigung müssen vermieden werden. Statistische Informationen, nicht zuletzt zur Frage der Prognose sind mit besonderer Sensibilität zu vermitteln. Um das bestmögliche Behandlungsergebnis zu erreichen, muss auch der Bedarf zur eigenverantwortlichen Kooperation des Patienten herausgearbeitet werden. Hier ist auch auf die neuen Konzepte zur rascheren postoperativen Rekonvaleszenz unter aktiver Mitwirkung des Patienten einzugehen („ERAS“). Hilfreich ist die Verfügbarkeit von schriftlichen Informationen und Abbildungen, die ebenfalls in dem Patienten verständlicher Sprache abgefasst sein müssen. Geeignet wird hierfür z. B. die in Erstellung befindliche Laienversion (Patientenleitlinie) dieser Leitlinie sein (Download unter: http:// leitlinienprogramm-onkologie.de/Patientenleitlinien.8.0.html).

Die Aufklärung sollte in einer von Arzt und Patient partizipativ und gemeinsam getragenen Entscheidung zum therapeutischen Vorgehen münden („shared decision-making“). Der Wunsch nach einer Zweitmeinung ist konstruktiv zu akzeptieren und durch zeitnahe Bereitstellung der medizinischen Patientenunterlagen (diagnostische Befunde, Arztbrief, Operationsbericht, Computertomografie auf CD-Rom) zu unterstützen. Zu jeder Zeit muss der Patient Einsicht in die Krankenunterlagen erhalten können.

\section{Risikofaktoren}

In $>$ Abb. 1 sind die bisher bekannten und nachfolgend in Detail dargestellten Risikofaktoren für die Entstehung eines Ösophaguskarzinoms zusammengefasst.

\subsection{Rauchen}

\begin{tabular}{|l|l|l|}
\hline $\begin{array}{l}\text { 4.1. } \\
\text { Evidenzbasiertes Statement }\end{array}$ & $\begin{array}{l}\text { geprüft } \\
\mathbf{2 0 1 8}\end{array}$ \\
\hline $\begin{array}{l}\text { Level of } \\
\text { Evidence } \\
\mathbf{3 a}\end{array}$ & $\begin{array}{l}\text { Rauchen erhöht das Risiko für Ösophaguskarzinome. Dies } \\
\text { gilt für Plattenepithelkarzinome und für Adenokarzinome } \\
\text { des Ösophagus und des ösophagogastralen Übergangs. }\end{array}$ \\
\hline $\begin{array}{l}\text { Konsens- } \\
\text { stärke }\end{array}$ & Starker Konsens (100\%) \\
\hline
\end{tabular}

\section{Hintergrund}

In einer multizentrischen Fall-Kontroll-Studie aus Taiwan wurde der Einfluss von Rauchen, Alkohol und Betelnüssen auf die Entstehung von Plattenepithelkarzinomen und deren Lokalisation im Ösophagus untersucht [8] Rauchen steigerte das Karzinomrisiko um das 5,9- bis 8,5-Fache, wobei das höchste Risiko im mittleren Ösophagus bestand. Raucher erkrankten 2,7- bis 6,2-mal häufiger an einem Plattenepithelkarzinom als Nichtraucher. Der gleichzeitige Genuss von Zigaretten und Alkohol wirkte synergistisch und erhöhte das Risiko auf das 10- bis 23,9-Fache im Vergleich zu abstinenten Nichtrauchern.

In einer bevölkerungsbasierten Fall-Kontroll-Studie bei Patienten mit Reflux wurde u. a. der zusätzliche Einfluss von Rauchen auf das Karzinomrisiko analysiert [9] 365 bzw. 426 Patienten mit Adenokarzinomen des Ösophagus und des ösophagogastralen Übergangs sowie 303 Patienten mit Plattenepithelkarzinom wurden Kontrollen aus einem Bevölkerungsregister gegen- 
übergestellt. Raucher mit Refluxbeschwerden wiesen für alle drei Tumorentitäten ein höheres Risiko als Nichtraucher auf.

Eine auf 30 Fall-Kontroll- und 3 Kohorten-Studien basierende Metaanalyse errechnete für Raucher ein relatives Risiko von 1,85 (95\% Konfidenzintervall 1,59-2,15), an einem Adenokarzinom des Ösophagus oder der Kardia zu erkranken [7].

Zwei neuere Fall-Kontroll-Studien bestätigen Rauchen als Risikofaktor für das Ösophaguskarzinom. In einer Studie aus Indien wurden 703 Patienten mit Plattenepithelkarzinomen des Ösophagus mit 1664 Kontrollen verglichen [10]. Es fand sich eine dosisabhängige Risikoerhöhung durch „secondhand“ Rauchen.

Die andere Fall-( $n=670)$ Kontroll-( $n=1188)$-Studie fokussierte auf Tabak- und Alkohol- konsum [11]. Im Vergleich zu Nichtrauchern zeigten weibliche und männliche Raucher ein 3,5-4-fach erhöhtes Karzinomrisiko (Frauen: OR 3,45; 95 \% Cl 2,47-4,82; Männer: OR 4,11; $95 \% \mathrm{Cl} 2,55-6,65)$. Bei Konsum von mehr als $14 \mathrm{~g}$ Tabak täglich stieg das Risiko auf den Faktor 6.

\subsection{Alkohol}

\begin{tabular}{|l|l|l|}
\hline $\mathbf{4 . 2 .}$ & Evidenzbasiertes Statement & $\begin{array}{l}\text { modifiziert } \\
\mathbf{2 0 1 8}\end{array}$ \\
\hline $\begin{array}{l}\text { Level of } \\
\text { Evidence } \\
\mathbf{2 b}\end{array}$ & $\begin{array}{l}\text { Alkohol erhöht das Risiko für Plattenepithelkarzinome des } \\
\text { Ösophagus. }\end{array}$ \\
\hline & Literatur: $[11-17]$ \\
\hline $\begin{array}{l}\text { Konsens- } \\
\text { stärke }\end{array}$ & Starker Konsens $(100 \%)$ \\
\hline
\end{tabular}

\section{Hintergrund}

Eine Metaanalyse von 40 retrospektiven und 13 prospektiven Studien aus USA, Asien, Australien und Europa errechnete folgende relative Risiken für ein Plattenepithelkarzinom des Ösophagus: leichter Alkoholkonsum (<12,5 g/Tag) 1,31 (95\% KI 1,10-1,57); moderater Alkoholkonsum (12,5 - 50 g/Tag) 2,27 (95\% KI 1,892,72); starker Alkoholkonsum ( $\geq 50 \mathrm{~g} / \mathrm{Tag}$ ) 4,89 (95\% KI 3,846,23 ) [12]. Analysierte man die prospektiven Studien alleine, waren die Ergebnisse nahezu identisch. Leichter Alkoholkonsum war insbesondere in Asien mit einem erhöhten Karzinomrisiko assoziiert, was man mit dem Einfluss genetischer Faktoren erklärt. Eine weitere Metanalyse der gleichen Autorengruppe mit dem Fokus auf leichten Alkoholkonsum versus Alkoholabstinenz kam zu ähnlichen Ergebnissen: relatives Risiko für ösophageale Plattenepithelkarzinome 1,30 (95\% KI 1,09-1,56), statistisch signifikant nur in Asien (RR 1,49; 1,12 - 1,98) [13].

Für Adenokarzinome des Ösophagus und des ösophagogastralen Übergangs stellt Alkohol dagegen keinen gesicherten Risikofaktor dar. Hierfür sprechen eine Fall-Kontroll-Studie aus Australien [15] und eine gepoolte Analyse von 9 Fall-Kontroll- und 2 Kohortenstudien [14]. Eine Metaanalyse von 20 Fall-Kontrollund 4 Kohorten-Studien aus allen Kontinenten konnte keine Assoziation zwischen Alkoholkonsum und Adenokarzinom des Ösophagus oder der Kardia aufzeigen [16]. Dies galt auch für einen hohen Alkoholkonsum.
Die schon oben erwähnte Studie aus Südafrika bei SCC zeigt in ähnlicher Weise eine Risikoerhöhung von 2- bis 3,5-fach für Alkohol auf, wobei das Karzinomrisiko mit der Alkoholmenge assoziiert war: bei Verbrauch von mehr als $52,9 \mathrm{~g}$ täglich stieg das Risiko auf das 5-Fache an (Männer: OR 4,72; $95 \% \mathrm{Cl}$ 2,64 - 8,41; Frauen: OR 5,24; $95 \%$ Cl 3,34-8,23) [11]. Das Risiko erhöhte sich um das 8,5-Fache für Personen, die zusätzlich $>14 \mathrm{~g}$ Tabak pro Tag rauchten.

Eine bevölkerungsbasierte Studie aus Schweden umfasste u. a. 14518 Patienten mit Ösophaguskarzinom [17]. Fälle wurden mit dem ICD Code „alcohol use disorders“ identifiziert und mit dem schwedischen Krebsregister verlinkt. Alkoholmißbrauch bedingte im Vergleich zur Kontrollgruppe ein erhöhtes Ösophaguskarzinomrisiko $(\mathrm{SIR}=2,24 ; 95 \% \mathrm{Cl} 2,08-2,41)$. Dies galt sowohl für Plattenepithel- (2,89 (95\% Cl 2,65 - 3,15); bei Männern: 2,69; bei Frauen: 4,83) als auch für Adenokarzinome (1,20 (95\% Cl 1,01 1,41); bei Männern: 1,19 (95\% Cl 1,00-1,41); bei Frauen: 1,30 (95\% Cl 0,51-2,68). Wegen diesen Geschlechtsunterschieden und den angegebenen Konfidenzintervallen wurde diese Arbeit nicht als Grundlage für eine neue Empfehlung genommen.

\section{3. Übergewicht}

\begin{tabular}{|c|c|c|}
\hline 4.3. & Evidenzbasierte Statement & $\begin{array}{l}\text { geprüft } \\
2018\end{array}$ \\
\hline \multirow{2}{*}{$\begin{array}{l}\text { Level of } \\
\text { Evidence } \\
2 \mathbf{a}\end{array}$} & \multicolumn{2}{|c|}{$\begin{array}{l}\text { Übergewicht erhöht das Risiko für Adenokarzinome des } \\
\text { Ösophagus und des ösophagogastralen Übergangs. }\end{array}$} \\
\hline & \multicolumn{2}{|l|}{ Literatur: [18-24] } \\
\hline $\begin{array}{l}\text { Konsens- } \\
\text { stärke }\end{array}$ & \multicolumn{2}{|l|}{ Starker Konsens (100\%) } \\
\hline
\end{tabular}

\section{Hintergrund}

Aktuelle Übersichtsarbeiten und Metaanalysen bestätigen Übergewicht und Adipositas als Risikofaktor für Ösophaguskarzinome [19-21]. Eine Metaanalyse umfasste 22 Studien mit insgesamt 7945 Fällen mit Adenokarziom der Speiseröhre und/oder des Magens [21]. Das geschätzte relative Risiko (RR) für den gemeinsamen Endpunkt war bei Übergewicht (Body-Mass-Index (BMI) $25-30 \mathrm{~kg} / \mathrm{m} 2)$ 1,71 (95\% KI 1,50 - 1,96) und bei Adipositas $(\mathrm{BMI}>30 \mathrm{~kg} / \mathrm{m} 2)$ 2,34 (95\% KI 1,95-2,81). Die Beziehung war stärker für Adenokarzinome der Speiseröhre (BMI $\geq 30 \mathrm{~kg} / \mathrm{m} 2$ : $\mathrm{RR}=2,73(95 \% \mathrm{KI} 2,16-3,46)$ (10 Studien) als für die des Magens ( $R R=1,93$ (95\% KI 1,52-2,45) (9 Studien).

Die positive Assoziation zwischen Übergewicht bzw. Adipositas und dem Risiko für Adenokarzinome der Speiseröhre wurden auch in einer Übersichtsarbeit mit 6 Fall-Kontroll- und 6 Kohortenstudien konsistent gezeigt [20]. Guh et al. [19] berichten ebenfalls über Ösophaguskarzinome als Ko-Morbiditäten von Adipositas. Die relativen Risiken für Ösophaguskarzinome bei Übergewicht waren bei Frauen 1,15 (95\% KI 0,97 - 1,36) und bei Männern 1,13 (95\% KI 1,02 - 1,26), bei Adipositas lagen sie bei 1,20 (95\% KI 0,95-1,53) und 1,21 (95\% KI 0,97-1,52). 
Eine prospektive Kohortenstudie mit 218854 Teilnehmern (NIH-AARP Studie) untersuchte den Taillenumfang, d. h. eine abdominelle Fettverteilung, als Risikofaktor [22]. In der Nachbeobachtungszeit traten 253 Fälle mit einem Adenokarzinom der Speiseröhre auf. Sowohl Taillenumfang als auch das Taille-Hüftumfangs-Verhältnis waren positiv mit dem Risiko für Speisenröhrenkrebs assoziiert. Die Beziehung wurde auch für Personen mit Normalgewicht berichtet.

In einer retrospektiven Kohortenstudie mit insgesamt 1239 Patienten war ein hoher BMI mit einer erhöhten Dysplasieprävalenz im Barrett-Ösophagus assoziiert [23]. Allerdings wirkten sich BMI und eine Änderung des BMI nicht auf die Progression zur hochgradigen Dysplasie oder zum Adenokarzinom aus.

In einer großen bevölkerungsbasierten Kohortenstudie mit 9660 Patienten mit Barrett-Ösophagus erwies sich neben Alter und männlichem Geschlecht der BMI als Risikofaktor für eine Progression zum Adenokarzinom [24]. Interssant ist auch die Beobachtung, dass Statine karzinoprotektiv waren.

\subsection{Weitere Risikofaktoren}

\begin{tabular}{|c|c|c|}
\hline 4.4. & Evidenzbasiertes Statement & $\begin{array}{l}\text { geprüft } \\
2018\end{array}$ \\
\hline $\begin{array}{l}\text { Level of } \\
\text { Evidence }\end{array}$ & \multicolumn{2}{|c|}{$\begin{array}{l}\text { Eine Achalasie erhöht das Risiko für Plattenepithel- und } \\
\text { Adenokarzinome des Ösophagus. }\end{array}$} \\
\hline & \multicolumn{2}{|l|}{ Literatur: [25 - 27] } \\
\hline $\begin{array}{l}\text { Konsens- } \\
\text { stärke }\end{array}$ & \multicolumn{2}{|l|}{ Starker Konsens (100\%) } \\
\hline
\end{tabular}

\section{Hintergrund}

Eine Kohorte von fast 3000 Patienten, bei denen zwischen 1965 und 2003 die Entlassungsdiagnose einer Achalasie gestellt worden war, wurden mit der schwedischen Normalbevölkerung verglichen [26]. Die Achalasie ging mit einem erhöhten Risiko für Plattenepithelkarzinome (Standardisiertes Inzidenzverhältnis (SIR) 11,0; 6,0 - 18,4) und für Adenokarzinome (SIR 10,4; 3,8$22,6)$ des Ösophagus einher. Eine vorausgegangene chirurgische Myotomie erhöhte das Karzinomrisiko nicht.

Eine andere Langzeitstudie kommt aus den Niederlanden [25]. 448 Patienten (218 Männer und 330 Frauen; 51 (4 - 92) Jahre) mit zwischen 1975 und 2006 diagnostizierter Achalasie wurden nach pneumatischer Dilatation regelmäßig endoskopisch-bioptisch kontrolliert. Während einer Beobachtungszeit von 9,6 (0,1 - 32) Jahren wurden bei 15 Patienten (3,3\%) 11 (2-23) Jahre nach Diagnosestellung der Achalasie bzw. 24 (10-43) Jahre nach Symptombeginn ein Ösophaguskarzinom diagnostiziert. Das Karzinomrisiko des Achalasiekollektivs wurde mit der erwarteten Ösophaguskarzinomrate einer alters- und geschlechtsadjustierten Population aus dem niederländischen Krebsregister verglichen. Daraus ergab sich eine signifikant erhöhte Hazard Ratio von 28 (KI17 - 46) für das Auftreten eines Ösophaguskarzinoms bei Patienten mit Achalasie. Trotz der strukturierten endoskopi- schen Überwachung wurden die meisten Karzinome erst im fortgeschrittenen Stadium diagnostiziert.

Eine Auswertung des schwedischen Krebsfamilienregisters hat gezeigt, dass das Risiko für ein ösophageales Adenokarzinom erhöht ist, wenn ein Elternteil an einem Ösophaguskarzinom (SIR 2,60) und speziell einem Plattenepithelkarzinom (SIR 4,05) erkrankt war [27]. Auch wenn in Assoziationsstudien genetische Varianten identifiziert wurden, die mit einem erhöhten Risiko für Ösophaguskarzinome einhergehen, gibt es derzeit mit Ausnahme der sehr seltenen Tylose keine genetischen oder hereditären Faktoren mit Implikationen für die klinische Praxis.

\begin{tabular}{|l|l|l|}
\hline 4.5. & Konsensbasiertes Statement & $\begin{array}{l}\text { geprüft } \\
\mathbf{2 0 1 8}\end{array}$ \\
\hline EK & $\begin{array}{l}\text { Eine frühere Strahlentherapie im Hals-Thorax-Bereich kann } \\
\text { dosisabhängig das Risiko für ein späteres Ösophaguskarzi- } \\
\text { nom erhöhen. }\end{array}$ \\
\hline $\begin{array}{l}\text { Konsens- } \\
\text { stärke }\end{array}$ & Konsens (92\%) \\
\hline
\end{tabular}

\section{Hintergrund}

Die Empfehlung basiert auf einer selektiven Literaturauswahl durch die beteiligten Experten (Expertenkonsens). Es besteht ein signifikant erhöhtes, wenngleich absolut geringes Risiko für das Auftreten von Ösophaguskarzinomen nach früherer Bestrahlung im Hals-Thorax-Bereich (RR 2,0-8,3 [28-31]. Daten hierzu beziehen sich überwiegend auf Mammakarzinome und nur ausnahmsweise auf den Morbus Hodgkin. Das Risiko betrifft vor allem Plattenepithelkarzinome, die Daten für strahleninduzierte Adenokarzinome des Ösophagus sind indessen kontrovers.

In einer Fall-Kontroll-Studie mit 289748 Mammakarzinompatientinnen, die länger als 5 Jahre überlebt hatten, war eine Bestrahlung mit einer Dosis von mehr als 35 Gy mit einem erhöhten Ösophaguskarzinomrisiko assoziiert (OR 8,3; 95 \% KI 2,7 - 28). Das absolute Risiko war gering, es wurden 252 Fälle mit Ösophaguskarzinomen beobachtet [28].

Basierend auf den Daten eines bevölkerungsbasierten US-Registers der Jahre 1973 bis 2000 errechnete sich ein relatives Risiko von 2,83 (95\% KI 1,35-5,92) bzw. 2,17 (95\% KI 1,67-4,02) für Plattenepithelkarzinome des Ösophagus 5 - 9 bzw. mehr als 10 Jahre nach einer adjuvanten Bestrahlung von Patientinnen mit Mammakarzinom. Für ösophageale Adenokarzinome ergab sich kein erhöhtes Risiko [29]. Das Risiko von Ösophaguskarzinomen war in dieser Studie nicht nach brusterhaltender Operation und Tangentenbestrahlung, wohl aber nach Postmastektomie-Strahlentherapie erhöht.

Unter 11130 schweizerischen Patientinnen mit Mammakarzinom in den Jahren 1974- 2002 wurden 18 Fälle mit Ösophaguskarzinom im Vergleich zu 8,9 erwarteten beobachtet. Die SIR betrug 1,6 in den ersten 10 Jahren nach Bestrahlung, danach 3,3. Das Risiko für Plattenepithelkarzinome war dabei doppelt so hoch wie für Adenokarzinome (2,3 versus 1,3) [30].

Bei Mammakarzinompatientinnen mit zusätzlicher Bestrahlung bestand mehr als 15 Jahre nach der Bestrahlung ein erhöhtes relatives Risiko von $2,19(1,10-4,62)$ für das Auftreten von 
Ösophaguskarzinomen im Vergleich zu Mammakarzinom- Patientinnen mit alleiniger Operation und ohne Bestrahlung [31].

\begin{tabular}{|l|l|l|}
\hline 4.6. & Konsensbasiertes Statement & $\begin{array}{l}\text { geprüft } \\
\mathbf{2 0 1 8}\end{array}$ \\
\hline EK & $\begin{array}{l}\text { Stenosen nach Säure- und Laugenverätzungen erhöhen } \\
\text { das Risiko für das Ösophaguskarzinom. }\end{array}$ \\
\hline $\begin{array}{l}\text { Konsens- } \\
\text { stärke }\end{array}$ & Starker Konsens (100\%) \\
\hline
\end{tabular}

\section{Hintergrund}

Kontrollierte Studien zum Risiko eines Ösophaguskarzinoms bei Patienten mit kaustischen Ösophagusstenosen existieren nicht. Okonta et al. identifizierten im Rahmen einer systematischen Literatursuche 6 informative Publikationen [32]. Darin werden insgesamt 198 Patienten mit korrosiven Ösophagusstenosen nach Säure- oder Laugeningestion beschrieben, von denen 50 (25,3\%; 1,4 - 31,3\%) nach im Mittel 46,1 (25 - 58) Jahren ein Ösophaguskarzinom entwickelten.

\begin{tabular}{|l|l|l|}
\hline $\mathbf{4 . 7 .}$ & Evidenzbasiertes Statement & $\begin{array}{l}\text { modifiziert } \\
\mathbf{2 0 1 8}\end{array}$ \\
\hline $\begin{array}{l}\text { Level of } \\
\text { Evidence } \\
\mathbf{2 b}\end{array}$ & $\begin{array}{l}\text { Gastroösophagealer Reflux erhöht das Risiko für } \\
\text { Adenokarzinome des Ösophagus. }\end{array}$ \\
\hline & Literatur: $[9,33-38]$ \\
\hline $\begin{array}{l}\text { Konsens- } \\
\text { stärke }\end{array}$ & Starker Konsens $(100 \%)$ \\
\hline
\end{tabular}

\section{Hintergrund}

In einer schwedischen Fall-Kontroll-Studie lag bei Personen mit rezidivierenden Refluxsymptomen im Vergleich zu Personen ohne solche Symptome die Odds Ratio (OR) für ein Adenokarzinom des distalen Ösophagus bei 7,7 [33]. Je häufiger, schwerer und länger dauernd die Symptome waren, umso größer war das Karzinomrisiko.

In einer Fall-Kontroll Studie aus den USA wird eine höhere Prävalenz Reflux-assoziierter Symptome bei Patienten mit einem Karzinom des gastroösophagealen Übergangs als bei Kontrollpatienten gleichen Alters und gleichen Geschlechts beschrieben [34]. In einer weiteren bevölkerungsbasierten Fall-Kontroll-Studie aus den USA fand sich nach Berücksichtigung demografischer Faktoren ein 3-fach erhöhtes Risiko für ein Ösophaguskarzinom bei Refluxsymptomen (OR 3,61; 95 \% KI 2,49 - 5,25) [35]. Bei Vorliegen einer Hiatushernie war das Risiko 6-fach (OR 5,85; 95 \% KI $3,18-10,75)$ und bei Refluxsymptomen und Hiatushernie 8-fach (OR 8,11; 95 \% KI 4,75-13,87) erhöht.

In einer Studie aus den USA wurden Patienten, bei denen endoskopisch ein Adenokarzinom des Ösophagus oder ein LongSegment-Barrett-Ösophagus festgestellt worden war, nach ihren Refluxsymptomen befragt [36]. 61\% der Patienten mit Adenokarzinom des Ösophagus und $70 \%$ derer mit Barrett-Ösophagus berichteten über chronische Refluxsymptome mehr als 5 Jahre vor der Diagnosestellung.

In einer bevölkerungsbasierten Fall-Kontroll-Studie bei Patienten mit Reflux wurden 365 Patienten mit Adenokarzinom des Ösophagus, 426 Patienten mit einem Adenokarzinom des ösophagogastralen Übergangs und 303 Patienten mit einem ösophagealen Plattenepithelkarzinom entsprechende Kontrollen aus einem Bevölkerungsregister gegenübergestellt [9]. Häufige Refluxsymptome ( $\geq 1 \times /$ Woche) erhöhten das Risiko für ein Adenokarzinom des Ösophagus um das 6,4-Fache, für ein Adenokarzinom des ösophagogastralen Übergangs um das 4,6-Fache, und für das Plattenepithelkarzinom um das 2,2-Fache.

Rubenstein et al. führten eine systematische Literatursuche und Metaanalyse von fünf populationsbasierten Studien durch (Suche bis 2008). Die Metaanalyse der Studien zeigte, dass sich bei mindestens einmal wöchentlichen Refluxsymptomen das Karzinomrisiko um das 5-Fache und bei täglichen Symptome um das 7-Fache erhöht [37].

In einer auf der SEER-Datenbank basierenden Modellrechnung zeigte sich, dass bei Männern 13,4\% (11,4-17,3\%) der Fälle mit Adenokarzinom des Ösophagus auf eine symptomatische GERD zurückzuführen sind. Für Frauen errechneten sich 13,6 \% (12,5$15,9 \%)[38]$.

\begin{tabular}{|l|l|l|}
\hline $\mathbf{4 . 8 .}$ & Evidenzbasierte Statement & $\begin{array}{l}\text { geprüft } \\
\mathbf{2 0 1 8}\end{array}$ \\
\hline $\begin{array}{l}\text { Level of } \\
\text { Evidence } \\
\mathbf{2 b}\end{array}$ & $\begin{array}{l}\text { Der Barrett-Ösophagus erhöht das Risiko für Adenokarzi- } \\
\text { nome des Ösophagus. }\end{array}$ \\
\hline & Literatur: $[39-49]$ \\
\hline $\begin{array}{l}\text { Konsens- } \\
\text { stärke }\end{array}$ & Starker Konsens (100\%) \\
\hline
\end{tabular}

\section{Hintergrund}

In einer populationsbasierten Kohortenstudie aus Dänemark wurden 11028 Patienten, bei denen Barrett-Ösophagus in den Jahren 1992 bis 2009 diagnostiziert worden war, über einen Zeitraum von durchschnittlich 5,2 Jahren nachverfolgt [39]. Innerhalb des ersten Jahres nach der Indexendoskopie wurden 131 ösophageale Adenokarzinome beobachtet. In den darauffolgenden Jahren traten 66 neue Adenokarzinome auf (1,2 Fälle/1000 Patientenjahre). Im Vergleich zur Normalbevölkerung hatten die Patienten mit Barrett-ösophagus ein 11-fach erhöhtes Risiko für ein Adenokarzinom des Ösophagus (RR 11,3, 95 \% KI 8,8-14,4). Das jährliche Risiko betrug nur 0,12\% (95\% KI 0,09-0,15). Der Nachweis einer low-grade intraepithelialen Neoplasie in der Indexendoskopie erhöhte das Karzinomrisiko (5,1 Fälle/1000 Patientenjahre).

In einer multizentrischen Kohortenstudie aus den USA entwickelten 18 von 1204 Patienten mit Barrett-Ösophagus innerhalb von 5,5 Jahren ein Adenokarzinom (0,27\%/Jahr) und 32 eine high-grade Neoplasie (0,48\%/Jahr) [40]. Die Inzidenz der Barrettneoplasie betrug insgesamt 0,63\%/Jahr. Patienten mit nichtdysplastischem Barrett-Ösophagus waren 5 und 10 Jahre nach 
der Diagnosestellung in 98,6\% und 97,1\% der Fälle karzinomfrei. Die Länge des Barrett-Ösophagus korrelierte mit dem Neoplasierisiko (<6 cm: 0,09\%, $\geq 6 \mathrm{~cm}: 0,65 \%, p=0,001)$. In einer Analyse von 1175 Patienten dieser Kohorte wurde der Einfluss der Länge des Barett-Ösophagus auf das Karzinomrisiko genauer analysiert [41]. Das jährliche Neoplasie/Karzinomrisiko war in Abhängigkeit von der Länge des Barrett-Ösophagus wie folgt: $\leq 3 \mathrm{~cm}: 0,3 \%$; 4-6 cm: 0,97\%; 7 - 9 cm: 1,26\%; $10-12 \mathrm{~cm}: 1,64 \%$; $\geq 13 \mathrm{~cm}$ : $2,4 \%$. Rechnerisch betrug die Risikosteigerung für jeden weiteren Zentimeter des Barett-Ösophagus $28 \%(p=0,01)$.

In einer weiteren multizentrischen Kohortenstudie aus den USA wurden 1401 Patienten mit Barrett-Ösophagus ohne intraepitheliale Neoplasie im ersten Jahr der Nachbeobachtung endoskopisch nachverfolgt [42]. In der logistischen Regressionsanalyse korrelierte die Anzahl der Überwachungsendoskopien, die einen nicht-dysplastischen Barrett-Ösophagus ergaben, negativ mit dem jährlichen Karzinomrisiko ( $n=1: 0,32 \% ; n=2: 0,27 \% ; n=3$ : $0,16 \% ; n=4: 0,20 \% ; n=5: 0,11 \%, p=0,03)$.

In einer Metaanalyse von 57 Studien mit 11434 Patienten mit nicht-dysplastischem Barrett-Ösophagus und einer Nachbeobachtungszeit von 58547 Patientenjahren wurde eine Inzidenz des Ösophaguskarzinoms von 0,33\% pro Jahr errechnet [43]. Bei Patienten mit einem short-segment-Barrett-Ösophagus betrug das Karzinomrisiko nur 0,19\%. In 16 Studien, die Informationen zur Mortalität enthielten, war die Todesursache in 56 Fällen das Ösophaguskarzinom und in 684 Fällen eine Ösophagus-unabhängige Erkrankung.

In einer populationsbasierte Kohortenstudie aus England wurden 8448 Patienten mit einem Barrett-Ösophagus prospektiv ab dem ersten Jahr der Nachbeobachtung identifiziert und nachverfolgt [44]. Die Mortalität durch ein Barrett-assoziiertes Adenokarzinom betrug nach 5 Jahren $0,5 \%$ und nach 10 Jahren $2 \%$. Die häufigsten Todesursachen waren kardiovaskuläre Erkrankungen (31,6\%), andere Malignome (23,5\%), oder respiratorische Erkrankungen $(15,1 \%)$. Ösophaguskarzinome waren nur in 4,5\% der Fälle die verantwortliche Todesursache.

In der S2k-Leitlinie „Gastroösophageale Refluxkrankheit“ der Deutschen Gesellschaft für Gastroenterologie, Verdauungs- und Stoffwechselkrankheiten werden - basierend auf einem Expertenkonsens - endoskopische Überwachungsintervalle von Patienten mit Barrett-Ösophagus in Abhängigkeit vom Vorhandensein intraepithelialer Neoplasien empfohlen (keine intraepitheliale Neoplasie: Kontrolle nach 1 Jahr, bei Bestätigung kann ein Kontrolle alle 3 bis 4 Jahre erfolgen; leichtgradige intraepitheliale Neoplasie: falls sichtbar, endoskopische Therapie, sonst im 1. Jahr halbjährlich, dann jährlich, alternativ Ablation mit RFA; hochgradige intraepitheliale Neoplasie: endoskopische Therapie) [45].

In einer Fall-Kontroll-Studie entwickelten 55 Barrett-Patienten während eines Follow-ups von 17743 Patientenjahren ein Adenokarzinom [46]. Daraus berechnete sich eine Karzinomprogressionsrate von 0,3\%/Jahr. Zunehmendes Alter $(1,03,95 \% \mathrm{Cl}$ $1,01-1,05, p=0,005)$, männliches Geschlecht $(3,06,95 \% \mathrm{Cl}$ $1,50-6,24, p=0,002)$ und die Einnahme von Asthmamitteln $(2,91,95 \% \mathrm{Cl} 1,10-7,68, \mathrm{p}=0,0314)$ konnten als Risikofaktoren identifiziert werden.

Das Risiko für ein Adenokarzinom bei nicht-dysplastischem Barrett nimmt mit der Zeit und der Zahl der Endoskopien nicht ab. Zu diesem Ergebnis kommt eine retrospektive Kohortenstudie aus den USA, in die 28561 Patienten mit einem zwischen 2004 und 2009 neu diagnostizierten Barrett eingingen [47]. Das mediane Follow-up betrug 4,9 Jahre. Die Inzidenzrate der Adenokarzinome stieg mit jeder weiteren Endoskopie nach vorherigem endoskopischen Negativbefund an: RR 1,43; 1,25-1,64. Im Vergleich zur ersten Kontrollendoskopie war das Karzinomrisiko nach der fünften Kontrolle 9-fach höher. Ebenso stieg die Inzidenzrate mit der Zeit an: RR 1,49 bei Vergleich nach 5 Jahren mit der Inzidenz im zweiten Beobachtungsjahr.

Im Zeitraum von 2000 bis 2012 wurden im UK 12312 inzidente Fälle mit BE identifiziert, von denen 40 (0,3\%) im Verlauf ein Adenokarzinom entwickelten [48]. In den NL waren es 1383 BE-Patienten, von denen 5 (0,4\%) ein Karzinom entwickelten. In beiden Kohorten stieg das Barrett-Risiko linear mit dem Alter an. In den Jahren 2000 - 2003 nahm die Inzidenz des BE um 35 \% bei Männern und $41 \%$ bei Frauen zu, ab 2012 blieben die Inzidenzraten stabil.

Auf der Basis des holländischen Pathologieregisters wurden 1579 Patienten mit BE weiter verfolgt, von denen bei 161 in der Indexendoskopie eine LGD von einem Zweitpathologen bestätigt worden war [49]. In dieser Gruppe betrug die Inzidenz einer HGD bzw. eines Karzinoms 5,18/100 Personenjahre. Im Vergleich dazu betrug die Inzidenz nur 1,85/100 Personenjahre, wenn die LGD nicht durch einen Zweitpathologen verifiziert worden war.

\begin{tabular}{|l|l|l|}
\hline 4.9. & Konsensbasiertes Statement & $\begin{array}{l}\text { geprüft } \\
\mathbf{2 0 1 8}\end{array}$ \\
\hline EK & $\begin{array}{l}\text { Patienten mit Plattenepithelkarzinomen des Ösophagus } \\
\text { besitzen ein erhöhtes Risiko für synchrone und metachrone } \\
\text { Kopf-/Halstumoren und umgekehrt. }\end{array}$ \\
\hline $\begin{array}{l}\text { Konsens- } \\
\text { stärke }\end{array}$ & Starker Konsens (96\%) \\
\hline
\end{tabular}

\section{Hintergrund}

Eine Literaturübersicht ergibt für Patienten mit Kopf-HalsKarzinomen ein Risiko von 5-14\%, simultan oder metachron an einem Plattenepithelzweitkarzinom der Speiseröhre zu erkranken [50]. Umgekehrt beträgt das Risiko für pharyngolaryngeale Zweitneoplasien bei Patienten mit Plattenepithelkarzinom des Ösophagus ebenfalls $9-11 \%$. Es bietet sich daher an, diesen Personenkreis interdisziplinär zu betreuen und zu überwachen, zumal durch prospektive Studien gezeigt werden konnte, dass durch endoskopische Vorsorgeuntersuchungen eine Früherkennung von Zweitneoplasien und eine kurative Therapie möglich sind [51].

Zu etwas anderen Ergebnissen kam eine bevölkerungsbasierte Fall-Kontroll-Studie in Schweden insofern, als eine Erkrankung der Mundhöhle mit keinem erhöhten Risiko für ein Plattenepithelkarzinom des Ösophagus assoziiert war, wenn man Alkohol und Tabakkonsum berücksichtigte (OR 1,1; 95 \% KI 0,8-1,7) [52]. Erstaunlicherweise bestand nach Berücksichtigung dieser Einflussfaktoren jedoch ein erhöhtes Risiko für ösophageale Adenokarzinome. 


\section{Prävention}

\begin{tabular}{|l|l|l|}
\hline $\mathbf{5 . 1 .}$ & Konsensbasiertes Statement & $\begin{array}{l}\text { geprüft } \\
\mathbf{2 0 1 8}\end{array}$ \\
\hline $\mathbf{E K}$ & $\begin{array}{l}\text { Eine Empfehlung zur medikamentösen Prävention der } \\
\text { Entwicklung eines Ösophaguskarzinoms kann nicht } \\
\text { gegeben werden. }\end{array}$ \\
\hline $\begin{array}{l}\text { Konsens- } \\
\text { stärke }\end{array}$ & \begin{tabular}{l} 
Starker Konsens (96\%) \\
\hline
\end{tabular} \\
\hline
\end{tabular}

\section{Hintergrund}

ASS und nichtsteroidale Antirheumatika (NSAR) können in der primären Prävention nicht empfohlen werden. Diese Ansicht äußerte auch die britische Leitlinie zum Management des Ösophagus- und Magenkarzinoms, die keine etablierte Rolle für eine Chemoprävention bei Karzinomen des oberen Gastrointestinaltraktes sah [53]. Zum Zeitpunkt dieser Aussage lagen allerdings zwei Studien noch nicht vor [54, 55].

Eine Analyse von acht randomisierten Studien mit über 25000 Patienten, die zur Prävention kardiovaskulärer Ereignisse ASS über einen Zeitraum von mindestens vier Jahren erhalten hatten (751200 mg/d), kam zu dem Schluss, dass die tägliche ASS-Einnahme das Risiko an einem Karzinom zu versterben um $21 \%$ (OR 0,79; $95 \% \mathrm{KI} 0,68-0,92 ; \mathrm{p}=0,003)$ reduziert [54]. Auf der Basis individueller Daten von 23535 Patienten aus sieben Studien errechnete sich ein Vorteil zugunsten von ASS bereits nach fünf Jahren Einnahme. Der Nutzen nahm mit der Dauer der Medikation zu und war unabhängig von der ASS-Dosis (75 mg oder mehr). Die Autoren weisen auf die Konsistenz der Daten in den verschiedenen Studienpopulationen hin und leiten daraus die allgemeine Gültigkeit ihrer Ergebnisse ab. Selbst unter Berücksichtigung der gastrointestinalen Toxizität des ASS bedingt die Medikation eine Abnahme der Gesamtsterblichkeit um $10 \%$.

Eine systematische Literaturanalyse verglich die Ergebnisse von Fall-Kontroll- und Kohortenstudien der Jahre 1950 bis 2011, die über eine Assoziation zwischen ASS und Karzinomrisiko berichteten, mit denen von randomisierten Studien [55]. In FallKontroll-Studien war die regelmäßige Einnahme von ASS mit einer Risikominderung von 38 \% für Ösophagus-, Magen-, Gallenwegs und kolorektale Karzinome assoziiert, wobei dies mit den Daten randomisierter Studien gut übereinstimmte. Observationsstudien zeigten ähnliche Ergebnisse und darüber hinaus ein reduziertes Risiko für Fernmetastasen um $31 \%$.

Dennoch konnte man sich in dieser wie auch in den S3-Leitlinien zum Magenkarzinom und kolorektalen Karzinom bislang nicht zu einer Empfehlung im Sinne der medikamentösen Primärprävention entschließen. Ergänzend gibt es zwischenzeitlich eine neue Kosten-Nutzen-Analyse für gesunde Individuen im Alter von 50 bis 65 Jahren [56]. Sie zeigt, dass eine ASS-Einnahme über 10 Jahre mit einer Reduktion des relativen Risikos für Krebs, Herzinfarkt oder Schlaganfall um $7 \%$ für Frauen und $9 \%$ für Männer einhergeht. Die Gesamtsterblichkeit über 20 Jahre nimmt um $4 \%$ ab. Allerdings muss berücksichtigt werden, dass in der randomi- sierten Women's Health Study (39876 Frauen aus der Gesundheitsbranche randomisiert von 4/1993 - 1/1996; 100 mg Aspirin alle 2 Tage; medianes Follow-up 17,5 Jahre) bezüglich der totalen Karzinominzidenz, der Mamma- und der Bronchialkarzinominzidenz kein Unterschied zwischen beiden Gruppen bestand und dass selbst unter niedrig dosiertem Aspirin gastrointestinale Blutungen signifikant häufiger (HR 1,14) auftraten [57].

Inwieweit orale Bisphosphonate das Risiko für Ösophaguskarzinome beeinflussen, kann derzeit nicht abschließend beurteilt werden. Die Datenlage hierzu ist widersprüchlich [58-62]. Nach der jüngsten Fall-Kontroll-Studie fand sich ein geringfügig erhöhtes Risiko für Bisphosphonate (OR 1,34; 95 \% Cl 1,03 - 1,74), ohne dass eine Dosisabhängigkeit festgestellt werden konnte [63].

Auch für Antioxidantien als Nahrungsergänzungsmittel kann keine Empfehlung ausgesprochen werden. In einer Metaanalyse von 20 randomisierten Studien mit über 200000 Teilnehmern fand sich keine Evidenz für eine Prävention gastrointestinaler Karzinome durch Betakarotin, Vitamin A, C, E und Selen (OR 0,94; $95 \%$ KI 0,83-1,06) [64].

Körperliche Aktivität kann unter allgemeinen Gesundheitsaspekten und der Prävention empfohlen werden. Eine bevölkerungsbasierte Fall-Kontroll-Studie ließ einen mäßigen protektiven Effekt von körperlicher Aktivität auf das Adenokarzinom des Ösophagus erkennen [65].

\begin{tabular}{|l|l|l|}
\hline $\mathbf{5 . 2 .}$ & Konsensbasiertes Statement & $\begin{array}{l}\text { geprüft } \\
\mathbf{2 0 1 8}\end{array}$ \\
\hline EK & $\begin{array}{l}\text { Eine hohe Aufnahme von Obst und Gemüse kann zu einer } \\
\text { Risikosenkung des Ösophaguskarzinoms beitragen. }\end{array}$ \\
\hline $\begin{array}{l}\text { Konsens- } \\
\text { stärke }\end{array}$ & Starker Konsens (96\%) \\
\hline
\end{tabular}

\section{Hintergrund}

Eine systematische Evidenzbewertung der Beziehung zwischen Lebensmittelzufuhr und dem Risiko für Ösophaguskarzinome wurde im Ernährungsbericht der Deutschen Gesellschaft für Ernährung 2012 veröffentlicht [66]. Die inverse Beziehung zwischen dem Verzehr von Obst und Gemüse und dem Risiko der Entwicklung eines Plattenepithelkarzinoms der Speiseröhre wurde als wahrscheinlich eingestuft, die positive Beziehung mit dem Verzehr von rotem Fleisch und Fleischerzeugnissen als möglich. Die Bewertung stützt sich auf prospektive Kohortenstudien und Metaanalysen, die bis 2011 veröffentlicht wurden.

Eine aktuellere Metaanalyse zum Verzehr von Obst und Gemüse wertete 27 Fall-Kontroll- und 5 Kohortenstudien aus [67]. Eine statistisch signifikant inverse Beziehung zwischen dem Verzehr von Gemüse und Obst und dem Risiko für ein Plattenepithelkarzinom der Speiseröhre wurde gezeigt. Die Betrachtung der Kohortenstudien alleine zeigte eine inverse Beziehung für Obst, jedoch nicht für Gemüse.

Kohortenstudien und Metaanalysen zum Verzehr von Fleisch und Fleischerzeugnissen und dem Risiko für Ösophagsukarzinome zeigen unterschiedliche Zusammenhänge [68 - 72]. Einige Studien belegen eine positive Assoziation zwischen dem Verzehr von rotem 
Fleisch oder Fleischerzeugnissen und dem Plattenepithelkarzinom, andere mit dem Adenokarzinom. Die Datenlage ist hier zu unsicher, um eine allgemein verbindliche Aussage treffen zu können.

\section{Primärdiagnostik und Staging inklusive Pathologie}

\subsection{Primärdiagnostik}

\begin{tabular}{|l|l|l|}
\hline $\mathbf{6 . 1 .}$ & Konsensbasierte Empfehlung & $\begin{array}{l}\text { geprüft } \\
\mathbf{2 0 1 8}\end{array}$ \\
\hline EK & $\begin{array}{l}\text { Alle Patienten mit neu aufgetretener Dysphagie, gastroin- } \\
\text { testinaler Blutung, rezidivierender Aspiration, rezidivie- } \\
\text { rendem Erbrechen, Dyspepsie, Gewichtsverlust und/oder } \\
\text { Inappetenz sollen einer frühzeitigen Endoskopie (ÖGD) } \\
\text { zugeführt werden. }\end{array}$ \\
\hline $\begin{array}{l}\text { Konsens- } \\
\text { stärke }\end{array}$ & \begin{tabular}{l} 
Starker Konsens (100\%) \\
\hline
\end{tabular} & \\
\hline
\end{tabular}

\begin{tabular}{|l|l|l|}
\hline 6.2. & Konsensbasierte Empfehlung & $\begin{array}{l}\text { geprüft } \\
\mathbf{2 0 1 8}\end{array}$ \\
\hline EK & $\begin{array}{l}\text { Bei der ÖGD sollen Biopsien aus allen suspekten Läsionen } \\
\text { genommen werden. Beim Barrett-Ösophagus sollen } \\
\text { zusätzlich 4-Quadranten-Biopsien entnommen werden. } \\
\text { Suspekte Areale sollen getrennt asserviert und histopa- } \\
\text { thologisch untersucht werden. }\end{array}$ \\
\hline $\begin{array}{l}\text { Konsens- } \\
\text { stärke }\end{array}$ & \begin{tabular}{l} 
Starker Konsens (100\%) \\
\hline
\end{tabular} & \\
\hline
\end{tabular}

\begin{tabular}{|l|l|l|}
\hline 6.3. & Konsensbasierte Empfehlung & $\begin{array}{l}\text { geprüft } \\
\mathbf{2 0 1 8}\end{array}$ \\
\hline EK & $\begin{array}{l}\text { Die ÖGD mittels hochauflösender Videoendoskopie } \\
\text { besitzt die höchste Sensitivität und Spezifität für den } \\
\text { Nachweis von Neoplasien des oberen Gastrointestinal- } \\
\text { traktes und soll daher als Standardverfahren zur Diag- } \\
\text { nosestellung eingesetzt werden. }\end{array}$ \\
\hline $\begin{array}{l}\text { Konsens- } \\
\text { stärke }\end{array}$ & \begin{tabular}{l} 
Starker Konsens (100\%) \\
\hline
\end{tabular} & \\
\hline
\end{tabular}

\section{Hintergrund}

Dysphagie-Beschwerden treten mit zunehmendem Alter gehäuft auf ( 7 - $10 \%$ Erwachsene $>50$ Jahre, $25 \%$ bei Krankenhauspatienten, 30 - $40 \%$ Insassen von Pflegeheimen) [73]. Anamnese und klinische Untersuchung geben häufig schon wichtige Hinweise auf neurogene, degenerative, pharyngeale oder medikamentös getriggerte Schluckstörungen, Störungen im HNO-Bereich, rezidivierende Aspiration/Pneumonien, psychogene Syndrome und andere, nicht-tumorös bedingte dysphagische Beschwerden. Beispiele für zuletzt genannte Ursachen sind die Refluxösophagitis, Hiatushernien, Ringe, eosinophile Ösophagitis, Divertikel (einschl. Zenker-Divertikel), subepitheliale Tumoren (Leiomyome, GIST) oder seltene Prozesse. Weltweit wird daher als „Good Clinical Practice“-Übereinkunft (GCP) bei Patienten mit sogenannten „Warn-" oder „Alarmsymptomen“ (progrediente/rezidivierende Dysphagie, Gl-Blutung, Gewichtsabnahme, rezidvierendes Erbrechen, rezidivierende Aspirationspneumonie, Inappetenz) frühzeitig zu einer hoch-auflösenden Video-Endoskopie des oberen Verdauungstraktes mit BiopsieEntnahme geraten.

Vorteile der hochauflösenden ÖGD sind die direkte Visualisierung und Lokalisierung mit Größenangabe suspekter Läsionen, Entnahme von Biopsien, die Oberflächenanalyse gesehener Veränderungen und die Einsatzmöglichkeit zusätzlicher optischer Verbesserungsverfahren (einschließlich HDTV-Auflösung, Vergrößerungsendoskopie, Chromoendoskopie sowie computerbearbeitete virtuelle Chromoendoskopie). Die Methode ist breit verfügbar und die Sicherheit heute hoch (Komplikationsrate einschl. Sedierung $1 \%$ /Letalität <0,1 \% [74]. Langjährige Praxiserfahrungen haben demonstriert, dass die ÖGD die höchste Sensitivität und Spezifität für den Nachweis von Neoplasien aller Stadien im oberen Verdauungstrakt besitzt. Bei hochgradig stenosierenden, hochsitzenden Tumoren kann ein dünnkalibriges Spezialendoskop (4 - 5 mm Durchmesser) hilfreich sein, um Passage-bedingte Komplikationen (Perforation, Blutungen) zu vermeiden.

\subsection{Erweiterte Diagnostik}

\begin{tabular}{|l|l|l|}
\hline $\mathbf{6 . 4 .}$ & Evidenzbasierte Empfehlung & $\begin{array}{l}\text { modifiziert } \\
2018\end{array}$ \\
\hline $\begin{array}{l}\text { Empfeh- } \\
\text { lungsgrad } \\
\mathbf{B}\end{array}$ & $\begin{array}{l}\text { Die Chromoendoskopie (Lugol'sche Lösung) oder die } \\
\text { computergestützten digitalen (Filter-) Verfahren sollten } \\
\text { bei Risiko-Patienten für ein Ösophaguskarzinom } \\
\text { (= anamnestisch Plattenepithelkarzinom des Mundes/ } \\
\text { Nase/Rachens/Bronchial-Systems, Ösophagus) eingesetzt } \\
\text { werden. }\end{array}$ \\
\hline $\begin{array}{l}\text { Level of } \\
\text { Evidence } \\
\mathbf{2 a}\end{array}$ & $\begin{array}{l}\text { Literatur: [75-78] } \\
\text { Konsens- } \\
\text { stärke }\end{array}$ & \begin{tabular}{l} 
Starker Konsens (96\%) \\
\hline
\end{tabular} \\
\hline
\end{tabular}

\begin{tabular}{|l|l|l|}
\hline $\mathbf{6 . 5 .}$ & Evidenzbasierte Empfehlung & $\begin{array}{l}\text { modifiziert } \\
\mathbf{2 0 1 8}\end{array}$ \\
\hline $\begin{array}{l}\text { Empfeh- } \\
\text { lungsgrad } \\
\mathbf{B}\end{array}$ & $\begin{array}{l}\text { Die Chromoendoskopie oder die computergestützten di- } \\
\text { gitalen (Filter-) Verfahren sollten zur Verbesserung der } \\
\text { Detektion von Dysplasien/Frühkarzinomen eingesetzt } \\
\text { werden. }\end{array}$ \\
\hline $\begin{array}{l}\text { Level of } \\
\text { Evidence } \\
\mathbf{1 b}\end{array}$ & \begin{tabular}{l} 
Literatur: $[76,79-86]$ \\
\hline $\begin{array}{l}\text { Konsens- } \\
\text { stärke }\end{array}$
\end{tabular} & Starker Konsens (100\%) \\
\hline
\end{tabular}




\section{Hintergrund}

Zum Nachweis eines Ösophaguskarzinoms ist die video-endoskopische Untersuchung mit gezielter Biopsie obligat. Bei Vorliegen einer hochgradigen, auch mit einem Pädiatergastroskop nicht passierbaren malignen Stenose ist die Zangenbiopsie aus dem proximalen Tumorbereich ggf. in Kombination mit einer Bürstenzytologie zum Karzinomnachweis sinnvoll. Beim Plattenepithelkarzinom kann durch topische Färbung mit Lugol'scher Lösung (Jodalkali) die Ausbeute neoplastischer Läsionen um ca. 30 \% erhöht werden (neoplastisches Gewebe ist Glykogen-arm und wird somit nicht angefärbt) [87-90]. Gerade bei Hochrisikopatienten (Alkoholiker, starke Raucher) und Patienten mit bereits bekannten Plattenepithelkarzinomen im Mund-Nasen-Rachenraum (hohes Risiko synchroner Läsionen im Ösophagus) ist die Chromoendoskopie nützlich [75-77]. Hierbei muss jedoch berücksichtigt werden, dass neben malignen Veränderungen auch entzündliche Schleimhaut-Veränderungen von der Färbung ausgespart bleiben, die Spezifität der Chromoendoskopie mit Lugol'scher (Jodalkali) Lösung folglich relativ niedrig ist. Andere computergestützte, endoskopisch einsetzbare digitale Filterverfahren wie das Narrow-Band-Imaging [79] - oder von anderen Herstellern verwendete, digitale „post-processing“-Verfahren wie FICE (Fujinon intelligent chromoendoscopy) und iSCAN - zielen darauf ab, durch digitale Änderung des Farbspektrums eine bessere Darstellung von Oberflächen oder Kapillargefäßen zu ermöglichen und somit die im Rahmen der Karzinogenese auftretende Neovaskularisierung als diagnostisches Kriterium zur Detektion früher Neoplasien zu nutzen („Virtuelle Chromoendoskopie“). Ein systematisches Review mit Metaanalyse von 14 überwiegend randomisierten Cross-over-Studien mit insgesamt 843 Patienten zur Frage „Advanced Imaging“ vs. Weißlicht-Endoskopie (WLE) zeigte eine im Mittel um $34 \%$ höhere diagnostische Ausbeute beim „Advanced Imaging“ gegenüber der WLE. Im Rahmen einer Subgruppenanalyse konnte dies auch für die „virtuelle Chromoendoskopie“ gezeigt werden. Direkte vergleichende Studien, die eine Überlegenheit der virtuellen Chromoendoskopie gegenüber der „klassischen“ Färbe-Spray-Chromoendoskopie beweisen würden, existieren derzeit nicht. Die vorhandenen Untersuchungen schlossen in der Regel auch Patienten mit Barrett-Ösophagus/IEN mit ein, wobei man aber von einer Übertragbarkeit der Ergebnisse ausgeht $[76,80]$. Ein Einsatz dieser Verfahren wird daher empfohlen.

Weitere, neue, endoskopische Verfahren wie die Endozytoskopie, die konfokale Laserscanning-Endomikroskopie oder die optische Kohärenztomografie ermöglichen - zumindest theoretisch - eine starke Vergrößerung der oberen Schleimhautschichten mit der Möglichkeit einer in-vivo Bilddarstellung und einer Auflösung, die vergleichbar mit einer konventionellen Histologie ist. Bisher sind die hierfür verwandten Systeme jedoch wenig verbreitet, die Evidenz ist bei nur wenigen Studien aus einzelnen Zentren limitiert und mit entsprechender Patientenselektion gering. Weitere randomisierte, kontrollierte Studien sind daher zur Einschätzung des praktischen Nutzens dieser Verfahren notwendig. Eine Empfehlung hierzu kann derzeit nicht getroffen werden.

Neue Arbeiten zeigen, dass die Detektionsraten für Präkanzerosen und für Dysplasien/IEN im Ösophagus durch konsequenten
Einsatz von fortgeschrittenen endoskopischen Zusatz-Verfahren bei der Endoskopie des OGI-Traktes - vor allem beim Barrett-Karzinom - verbessert werden. Deshalb sollten diese Verfahren in der Praxis großzügig - und routinemäßig - eingesetzt werden. Dazu zählen alle derzeit bekannten Verfahren von der lokalen Essigsäure-Applikation über die klassische Chromoendoskopie mit Aufsprühen von Farbstoffen, die aufwendige konfokale Laer-Endomikroskopie sowie die neuen virtuellen Chromo-Endoskopieverfahren (NBI, FICE, iSCAN).

So zeigt eine RCT-Crossover-Studie von Sharma et al. (2013) [81], dass der Einsatz des Narrow-band imaging (NBI) - Verfahrens ( $n=123$ Patienten) während der ÖGD gegenüber dem (ohnehin schon guten) HD-Weißlicht-Verfahren mit randomisierten Biopsien die histologische Diagnose einer intestinalen Metaplasie beim Barrett-Ösophagus mit weniger gezielten Biopsien vereinfachen kann (Evidenzlevel 1b). Die Dysplasie-Aufdeckrate war beim gezielten NBI-Einsatz ebenfalls besser (Sensitivität NBI $64 \%$, Spezifität 100\%, NPV 77\%, PPV $100 \%$ gegenüber Sensitivität $53 \%$, Spezifität $100 \%$, NPV $53 \%$ und PPV $100 \%$ ). Wenn bei der NBI-Endoskopie die Schleimhaut völlig regelmässig aussah, fanden sich hier in keinem Fall IEN bzw. Dysplasien.

Eine weitere Arbeit (Multicenter-RCT) von Canto $\mathrm{M}$ et al. (2014) [82] fand bei 192 Patienten, dass die in-vivo-Endomikroskopie (eCLE) unter real-time-Bedingungen mit gezielten Biopsien bei der HD-Weißlichtendoskopie (HDWLE) die Aufdeckung von neoplastischem Gewebe beim Barrett-Ösophagus deutlich verbessert (Evidenzlevel 1b). Dabei erlaubte die eCLE eine fast 5 -Fache Reduktion der Biopsiemenge pro Patient ( $p<0,0001)$. Beim Einsatz der eCLE konnten Neoplasien bei 40/119 Biopsien (34\%) nachgewiesen werden, während mit der HDWLE nur bei 41/580 Biopsien (7\%, p < 0,0001) IEN nachgewiesen werden konnten. Im klinischen Kontext bedeutete diese Verbesserung, dass von den 94 Patienten der HDWLE+eCLE-Gruppe bei $32 \mathrm{~Pa}$ tienten (34\%) der Dysplasie-Grad nach der eCLE geändert werden musste. Daher scheint der systematische Einsatz der eCLE unter Studienbedingungen die Detektion von Dysplasien/IEN signifikant zu verbessern. Es stellt sich jedoch die Frage, ob diese Vorteile in der klinischen Praxis den hohen Preis und die erheblichen Ressourcen für das Teamtraining rechtfertigen können, da sich diese Methode aufgrund dieser Limitationen bisher nicht weit verbreitet hat.

Eine Metaanalyse von 2016 [83] untersuchte alle Arbeiten zur konfokalen Laser-Mikroskopie (CLE) beim Barrett-Ösophagus und anderen Anwendungsgebieten im GI-Trakt. In 7 Studien wurden Anwendungen im Ösophagus untersucht. Dabei fand sich eine moderate Verbesserung der Detektionsraten von Dysplasien/IEN durch den Einsatz von CLE neben der HD-Weisslicht-Endoskopie. Für diese Technik werden dabei erhebliche Limitationen durch hohe Kosten, geringe Ausbreitung und der Notwendigkeit längerer Lernkurven und das Teamtraining gesehen. Die Metaanalyse von Gupta et al. (2014) [91] zum gleichen Thema fokussierte ausschliesslich auf den Einsatz der CLE zur Aufdeckung von HG-IEN oder Frühkarzinomen mittels „targeted biopsies“ bei Barrett-Patienten. Dabei wurde in 7 Studien eine gepoolte Sensitivität der CLE von 68 \% bei einer Spezifität von 88 \% (in der Per-Läsions-Analyse) bzw. in 4 Studien als Per-Patienen-Analyse von $86 \%$ und $83 \%$ gefunden. Aufgrund der Einschränkungen bezüglich der Sensitivi- 
tät und der positiven „Likelihood-Ratio“ (von 6,56 bzw. 5,61) wird in dieser Metaanalyse jedoch darauf hingewiesen, dass die CLE keineswegs den bisherigen Standard ersetzen kann und nur als Zusatzverfahren in der klinischen Praxis die individuellen Ergebnisse verbessern helfen kann.

Drei weitere Studien mit dem Evidenzlevel 2a deuten klar darauf hin, dass sowohl einfache Spray-Techniken, als auch vorhandene technische Verfahren zur genaueren/besser kontrastierten mukosalen Oberflächen-Betrachtung die Aufdeckung von Frühneoplasien bei Risiko-Patienten verbessert. So zeigt die Arbeit von Coletta et al. (2016) [85] in Form einer Metaanalyse (13 Studien) einmal mehr, dass die simple Essigsäurespülung beim Barrett-Ösophagus - aber nur in Verbindung mit der Histologie dieses Ziel erreicht. So erreicht man mit dieser Technik eine Sensitivität für HG-IEN und Frühkarzinome (Barrett) von $92 \%$ bei einer Spezifität von $96 \%$. Für die Aufdeckung einer nicht-dysplastischen, reinen Barrett-Metaplasie betrugen diese Werte $96 \%$ und $69 \%$ (Spezifität ohne Histologie). Daher sind Essigsäure-positive Befunde stets mit der Histologie aufgrund der Spezifitäts-Limitationen zu kombinieren.

Die Arbeit von Qumseya B] et al. (2013) [86] ist eine Metaanalyse, die 11 RCTs eingeschlossen hat. Dabei wurde untersucht, ob die klassische und die virtuelle Chromo-Endoskopieverfahren (z.B. FICE, NBI) die Ergebnisse der Weißlicht-Endoskopie bei der Aufdeckung von Ösophagus-Neoplasien beim Barrett-Ösophagus verbessen können. Dabei verbesserten die klassiche Chromoendoskopie und die virtuellen Verfahren die bioptisch überprüfte Aufdeckungsraten von HG-IEN und Frühkarzinomen um bis zu $34 \%(\mathrm{Cl} 20-56 \%, \mathrm{p}<0,0001)$. Subgruppenanalysen zeigten zudem, dass v. a. die virtuellen Chromoendoskopieverfahren diese diagnostische Verbesserung erlaubten (Evidenzlevel 2a).

Sehr interessant erscheint schliesslich noch die Arbeit von Chung CS et al. (2016) [78]. Diese besteht aus einer Metaanalyse von 16 Studien mit insgesamt 4918 Patienten. Das Evidenlevel dieser Arbeit beträt 2a. Die eingeschlossenen Patienten hatten alle ein primäres Karzinom aus dem Bereich der Kopf-Hals-Tumoren $(n=1781)$ oder ein hochsitzendes Plattenepithel-Ca. des Ösophagus $(n=2205)$ in der Vorgeschichte. Fragestellung war die Erkennung von sekundär-primären neoplastischen Läsionen im Ösophagus (=Zweitkarzinomen). Dazu wurden neben der Weißlicht-Endoskopie (WLE) das NBI-Verfahren und die klassische Chromoendoskopie mittels gesprühter Lugol'scher Lösung (Lugol-CE) im Ösophagus eingesetzt. Dabei erreichte die WLE alleine eine Sensitivität für Neoplasien von $53 \%$, während das NBI auf $87 \%$ und die Lugol-CE auf $88 \%$ kamen.

Die Spezifitäten betrugen dabei $99 \%$, $95 \%$ und $63 \%$. Die entsprechenden Areale unter der Receiver-Operator-Kurcve (ROC) betrugen $66 \%$ (WLE), $97 \%$ (NBI) und $82 \%$ (Lugol-CE). Diese Daten zeigen überzeugend, dass Patienten mit Plattenepithel-Karzinomen im Kopf-Hals-Bereich und dem Ösophagus im Verlauf hinsichtlich der Entwicklung von Zweitkarzinomen im Ösophagus überwacht werden sollten. Dazu sollten Zusatzmethoden wie NBI und CE großzügig eingesetzt werden.

\subsection{Staging des Ösophaguskarzinoms}

\begin{tabular}{|l|l|l|}
\hline $\begin{array}{l}\text { 6.6. } \\
\text { Evidenzbasierte Empfehlung }\end{array}$ & $\begin{array}{l}\text { modifiziert } \\
\mathbf{2 0 1 8}\end{array}$ \\
\hline $\begin{array}{l}\text { Empfeh- } \\
\text { lungsgrad } \\
\text { B }\end{array}$ & $\begin{array}{l}\text { Der endoskopische Ultraschall (EUS) sollte Bestandteil des } \\
\text { Stagings bei Patienten mit kurativer Therapieintention } \\
\text { sein. }\end{array}$ \\
\hline $\begin{array}{l}\text { Level of } \\
\text { Evidence } \\
\mathbf{1 b}\end{array}$ & Literatur: $[92-97]$ \\
\hline $\begin{array}{l}\text { Konsens- } \\
\text { stärke }\end{array}$ & Starker Konsens $(100 \%)$ \\
\hline
\end{tabular}

\section{Hintergrund}

Die Prognose des Ösophaguskarzinoms ist insgesamt schlecht, da der Tumor sich sehr früh lymphatisch und vaskulär ausbreitet und bereits ab der Infiltration der tiefen Submukosa (sm2) ein deutlich erhöhtes Risiko für eine lymphatische Ausbreitung aufweist [98]. Die Mehrzahl der Tumoren im Stadium cT2-T3 N0 M0 (prätherapeutisch) hatten in einer kürzlichen Analyse bei der OP bereits Lymphknotenmetastasen [99]. Daher wird ein möglichst genaues, prätherapeutisches Staging zur Therapiesteuerung immer wichtiger. Die Prognose der Patienten korreliert mit der lokalen Tumorinfiltrationstiefe (T-Kategorie) und dem Grad der lymphatischen Aussaat (N-Kategorie). Die Endosonografie (EUS) hat aufgrund ihrer hohen lokalen Ortsauflösung die höchste Treffsicherheit aller Verfahren zur Beurteilung der lokalen Infiltrationstiefe (T-Kategorie) und ist geeignet zur Beurteilung von Metastasen in regionären Lymphknoten bei Plattenepithel-Karzinomen des Ösophagus und bei Adenokarzinomen des ösophagogastralen Übergangs (AEG). Aufgrund ihrer relativ guten Treffsicherheit vor allem für eine höhere T-Kategorie (Sensitivität 91 - $92 \%$, Spezifität 94-99\%; > Tab. 6) und für das lokale N-Staging ( $\triangleright$ Tab. 6 ) ist sie das bildgebende Stagingverfahren der ersten Wahl ( $\triangleright$ Abb. 2). \ Tab. 6 zeigt eine Übersicht über die Staging-Ergebnisse des endoskopischen Ultraschalls für die T-Kategorie und das nodale Staging (N-Kategorie) von Ösophaguskarzinomen in vorhandenen Metaanalysen [92 - 95]. Die zusätzliche Verwendung von EUS-gesteuerten Feinnadel-Biopsien (FNP) kann die nodale Staging-Genauigkeit erhöhen, besonders bei der Abklärung zöliakaler, kleinkurvaturseitiger abdomineller LK-Metastasen (Sensitivität $97 \%$, Spezifität 96\%; siehe > Tab. 6). Die Ergebnisse haben erheblichen Einfluss auf das Patienten-Management. Leider haben bisher nur wenige Studien die EUS-FNP systematisch untersucht und die wenigen vorhandenen Studien weisen eine erhebliche Heterogenität auf. Die EUS stellt aber neben der Computertomografie das Verfahren der Wahl zum Staging des Ösophaguskarzinoms dar. Sie beeinflusst das Patientenmanagement und sollte - trotz bestehender Limitationen - großzügig eingesetzt werden, um frühzeitig eine Selektion von Patienten für eine endoskopische, primär operative, primär neo-adjuvante oder primär palliative Therapie zu ermöglichen.

Die vergleichende und interdisziplinäre Interpretation der Ergebnisse von EUS und Computertomografie verbessern die Stagingaussage gegenüber den Einzelbefunden. Die Leitliniengruppe 
> Tab. 6 Übersicht über die Staging-Ergebnisse des endoskopischen Ultraschalls (EUS) für die T- und N-Kategorien von Ösophaguskarzinomen (Sensitivität/Spezifität durch EUS/EUS-FNP).

\begin{tabular}{|c|c|c|c|}
\hline $\begin{array}{l}\text { Metaanalysen zum } \\
\text { EUS }\end{array}$ & $\begin{array}{l}\text { Anzahl Patienten/ } \\
\text { Studien }\end{array}$ & Sensitivität/Spezifität T-Kategorie & Sensitivität/Spezifität N-Kategorie \\
\hline $\begin{array}{l}\text { Thosani et al., } 2012 \\
\text { [92] }\end{array}$ & $1019 / 19$ & $\begin{array}{l}\text { Frühe Karzinome }{ }^{1} \text { (T1a/T1b): T1a: } 85 \% / 87 \% \\
\text { T1b: } 86 \% / 86 \%\end{array}$ & n. a. \\
\hline \multirow[t]{4}{*}{ Puli et al.,2008 [93] } & \multirow[t]{4}{*}{$2585 / 49$} & T1: $82 \% / 96 \%$ & EUS: $85 \% / 85 \%$ \\
\hline & & T2: $81 \% / 94 \%$ & \\
\hline & & T3: $91 \% / 94 \%$ & EUS-FNP: $97 \% / 96 \%$ \\
\hline & & T4: $92 \% / 97 \%$ & \\
\hline Van Vliet, 2008 [94] & $1841 / 31^{2}$ & & $\begin{array}{l}\text { EUS: } 80 \% / 70 \% \\
\text { Zöliakale Lymphknoten (früher „M1a“): } 85 \% / 96 \%\end{array}$ \\
\hline $\begin{array}{l}\text { Tranchemontagne, } \\
2009 \text { [95] }\end{array}$ & na/na & $\begin{array}{l}<\mathrm{T} 2:-175 \% \\
\geq \mathrm{T} 2: 97 \% / \mathrm{T} 4:-/ 99 \%\end{array}$ & $\begin{array}{l}76 \% / 67 \% \\
\text { Zöliakale Lymphknoten (früher „M1a“): } 75 \% / 94 \%\end{array}$ \\
\hline $\begin{array}{l}\text { Luo et al. } 2016 \\
{[95,97]}\end{array}$ & & $\begin{array}{l}\text { T1a: } 84 \% / 91 \% \\
\text { T1b: } 81 \% / 89 \% \\
\text { T4: } 84 \% / 96 \%\end{array}$ & n.a. \\
\hline
\end{tabular}

ist daher der Meinung, dass die Limitationen der einzelnen Stagingverfahren durch den individuell sinnvollen Einsatz einer Kombination der zur Verfügung stehenden diagnostischen Verfahren ein insgesamt gutes, präoperatives klinisches Staging ermöglicht ( $\triangleright$ Abb. 2).

Einschränkungen bestehen zum einen durch die UntersucherAbhängigkeit, zum anderen ist die Unterscheidbarkeit kleiner, mukosaler Prozesse methodisch begrenzt und die EUS-Diagnostik bei hochgradig stenosierenden Tumoren nur eingeschränkt möglich. Letzteres Problem ist klinisch zumeist zu vernachlässigen, da nicht passierbare Tumoren fast immer fortgeschrittenen ( $\geq T 3-$ ) Karzinomen entsprechen. Die vorhandenen Studien weisen eine hohe Heterogenität durch Verwendung verschiedenster Geräte, Prozessorgenerationen und Techniken auf (mechanische und digitale Radial- und Longitudinalscanner, Minisonden, nur wenige Studien mit Feinnadelpunktionen EUS-FNP), was die Vergleichbarkeit einschränkt. Die T-Kategorie wird im EUS ähnlich wie in der CT etwas häufiger überschätzt (Median EUS 10,6\%; CT 9,4\%) als unterschätzt (Median EUS 7,6\%; CT 6,7\%). Schwierigkeiten gibt es v. a. beim lokalen Staging von T2-Karzinomen, bei denen aufgrund von peritumoralen, entzündlichen Prozessen in bis zu $12,5 \%$ ein Overstaging beobachtet wird.

Der EUS kann nicht zwischen HGIEN und mukosalen Frühkarzinomen unterscheiden, kann aber bei dieser Fragestellung vor geplanter EMR oder ESD zum Ausschluss bisher nicht vermuteter, Wand-überschreitender Tumorprozesse bei vermeintlichem Frühkarzinom und ggf. zur Diagnostik von - bei Frühkarzinomen sehr seltenen - regionären Lymphknoten-Metastasen eingesetzt werden. In bis zu 8 - 12\% der Fälle in der Praxis kann man mittels EUS bildgebend bisher unbeschriebener, „okkulter“ Leberherde im linken Leberlappen sowie andere suspekte pathologische Befunde wie Aszites oder Pleuraverdi- ckungen erkennen und teilweise mittels EUS-FNP weiter abklären. Fakultativ können „distante“ (tumorferne) LK-Metastasen paraaortal oder parathyreoidal mittels EUS-FNP gesichert werden, v. a. zöliakale LK-Metastasen beim Plattenepithelkarzinom ( $\triangleright$ Abb. 2). Der Einsatz der Kontrastmittel-Endosonografie (KM-Endosonografie, CE-EUS) und der Ultraschall-Elastografie sind neue Methoden, okkulte und kleine Metastasen verbessert zu erkennen und ggf. die FNP zu verbessern, größere Datensammlungen liegen hierzu noch nicht vor.

Die N-Kategorie ist ein weiterer wichtiger prognostischer Parameter beim Ösophaguskarzinom. > Tab. 7 zeigt hierzu eine Übersicht der Leistungsfähigkeit des nodalen Stagings für verschiedene Verfahren. Allerdings ist hier zu bemerken, dass alle Studien bisher die alte UICC-Klassifikation von 2002 verwendeten, die distante „zöliakale“ Lymphknoten als Fernmetastasen („M1a“) wertete, was bei der aktuellen Version 7 von 2009 geändert wurde.

Eine neuere Arbeit (Evidenzgrad 1b) von Russell et al. [96] deutet darauf hin, dass ein konsequentes EUS-Tumorstaging bei Ösophagus-Karzinomen zu verbesserten Überlebensraten der mittels EUS untersuchten Patienten führt (ca. 3 Monate gegenüber der Vergleichgruppe). Möglicher Hauptgrund dafür ist, dass die mittels EUS-Staging untersuchten Patienten häufiger operiert wurden, was auf eine Selektion der Patienten aufgrund der dabei erhobenen Befunde hindeutet.

Eine weitere neue Arbeit von Luo LN et al. (2016) [97] bestätigt, daß die EUS im Vergleich zur CT die deutlich höhere „Staging accuracy“ bei Plattenepithel-Karzinomen aufweist. Diese ist besonders gut bei der Differenzierung eines T1a- von einem T1b-Karzinom (Sensitivität 84 und $83 \%$, Spezifität $91 \%$ und $89 \%$ ), sowie beim Staging eines T4-Tumors (Sensitivität 84\%, Spezifität 96\%).

Zum Staging des Ösophagus-Karzinoms wird der folgende diagnostische Algorithmus vorgeschlagen ( $\triangleright$ Abb. 2 ) 


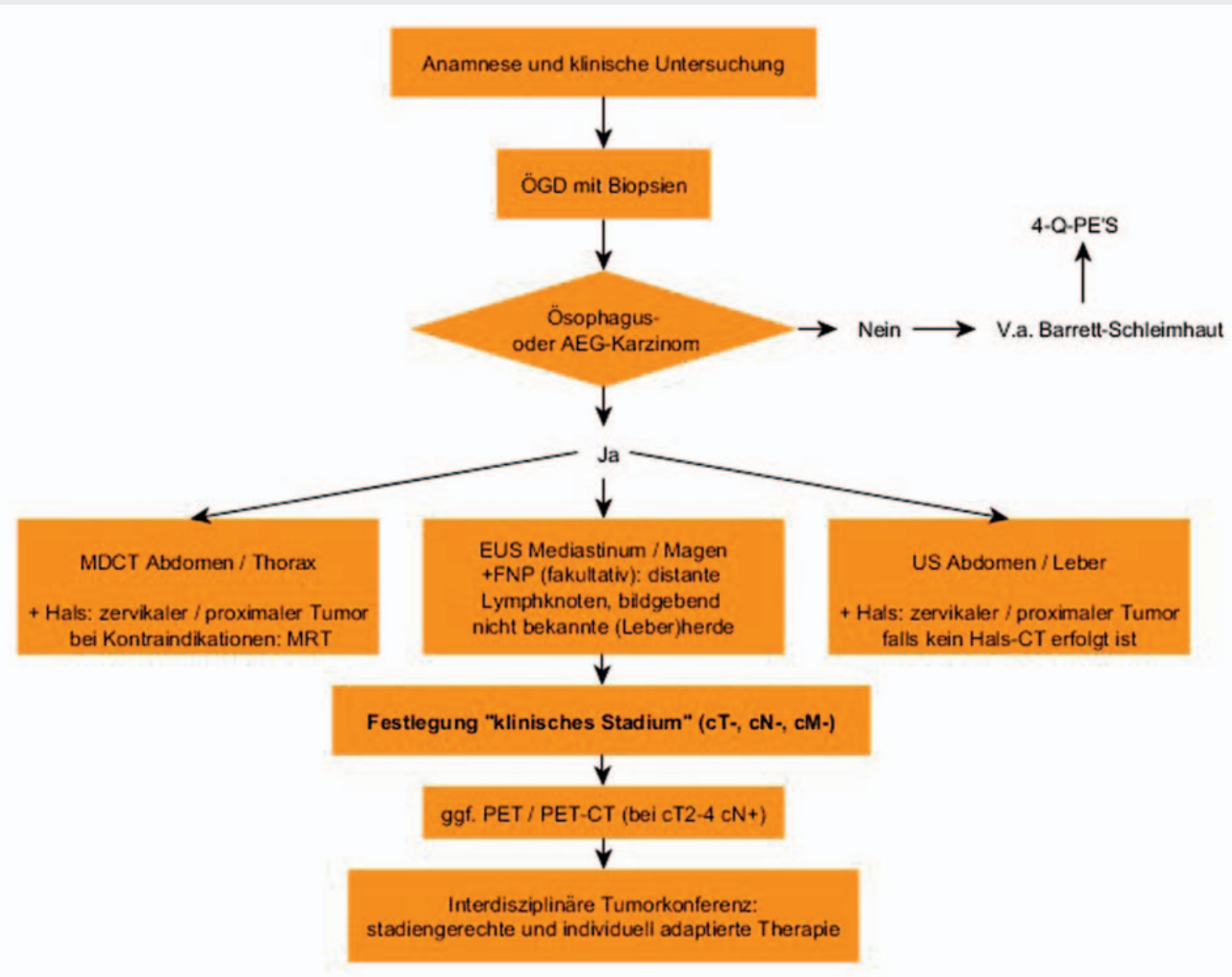

Abkürzungen:

AEG = Karzinome des gastro-ösophagealen Übergangs (adenocarcinoma of esophagogastric junction);

$\mathrm{CT}=$ Computertomographie; EUS = endoskopischer Ultraschall; FNP = Feinnadel Biopsie; MDCT = Multi-detector Computed Tomography; MRT = Magnetresonanztomographie; ÖGD = Ösophagogastroduodenoskopie; $\mathrm{PET}=$ Positronen-Emissions-Tomographie;

US = Ultraschall; 4-Q-PE'S = 4- Quadranten Probeexzisionen

Abb. 2 Algorithmus zum Staging des Ösophaguskarzinoms. [rerif]

- Tab. 7 Direkter Vergleich der eingesetzten Stagingverfahren bei der Aufdeckung von Lymphknoten-Metastasen [92 - 95].

\begin{tabular}{|l|l|l|l|}
\hline Methode & Gepoolte Sensitivität (95\% KI) & Gepoolte Spezifität (95\% KI) & Gepoolte Treffsicherheit (95\% KI) \\
\hline EUS-FNP & $81 \%(0,76-0,85)$ & $73 \%(0,63-0,80)$ & $77 \%(0,72-0,81)$ \\
\hline MDCT & $54 \%(0,48-0,61)$ & $87 \%(0,79-0,92)$ & $65 \%(0,60-0,70)$ \\
\hline FDG-PET & $52 \%(0,44-0,60)$ & $82 \%(0,65-0,92)$ & $69 \%(0,60-0,77)$ \\
\hline
\end{tabular}




\begin{tabular}{|l|l|l|}
\hline $\mathbf{6 . 7 .}$ & Konsensbasierte Empfehlung & $\begin{array}{l}\text { geprüft } \\
\mathbf{2 0 1 8}\end{array}$ \\
\hline $\begin{array}{l}\text { Empfeh- } \\
\text { lungsgrad } \\
\text { EK }\end{array}$ & $\begin{array}{l}\text { Die B-Bild-Sonografie sollte als erstes bildgebendes Ver- } \\
\text { fahren zum Ausschluss von Lebermetastasen eingesetzt } \\
\text { werden. }\end{array}$ \\
\hline $\begin{array}{l}\text { Konsens- } \\
\text { stärke }\end{array}$ & Konsens $(92 \%)$ \\
\hline
\end{tabular}

\section{Hintergrund}

Die Abdomensonografie ist als risikofreie, nicht-invasive, verfügbare und von Patienten akzeptierte Methode das bevorzugte initiale bildgebende Verfahren im Rahmen der Stagingdiagnostik. Die Untersucherexpertise ist ein wesentlicher Parameter für die Qualität der Ultraschall-Diagnostik. Die B-Bild-Sonografie weist eine Sensitivität von 53-81\% sowie eine Spezifität von 59-98\% bei der Detektion von Lebermetastasen, unabhängig von der zugrunde liegenden Tumorerkrankung, auf [100]. Eine Metaanalyse aus dem Jahre 2002, in die Ergebnisse von 9 publizierten Studien ( $N=509$ ) zur sonografischen Detektion von Lebermetastasen gastrointestinaler Tumoren eingegangen sind, fand eine gepoolte Sensitivität von 66 \% (95\% KI, 54 - 77\%) [101]. In neueren Studien wird eine Sensitivität von 77 bzw. $81 \%$ [102, 103] für den Nachweis von Lebermetastasen gastrointestinaler Tumoren durch die B-Bild-Sonografie berichtet.

Durch den zusätzlichen Einsatz der kontrastverstärkten Sonografie (contrast-enhanced ultrasound, CEUS) werden sowohl Sensivität als auch Spezifität deutlich erhöht. Der CEUS ist im klinischen Kontext hinsichtlich Spezifität und Sensitivität bei der Detektion von Lebermetastasen der CT und MRT vergleichbar [103 - 105]. Die Abgrenzung von Metastasen gegenüber primären malignen und benignen Tumoren der Leber gelingt mittels kontrastverstärkter Sonografie mit einer im Vergleich zur CT oder MRT identischen Genauigkeit von mehr als $90 \%$ [106 - 109].

\begin{tabular}{|l|l|l|}
\hline $\mathbf{6 . 8 .}$ & Konsensbasierte Empfehlung & $\begin{array}{l}\text { geprüft } \\
\mathbf{2 0 1 8}\end{array}$ \\
\hline EK & $\begin{array}{l}\text { Die B-Bild-Sonografie des Halses kann ergänzend zum } \\
\text { Ausschluss von zervikalen Lymphknotenmetastasen zum } \\
\text { Staging eingesetzt werden. }\end{array}$ \\
\hline $\begin{array}{l}\text { Konsens- } \\
\text { stärke }\end{array}$ & Konsens $(89 \%)$ \\
\hline
\end{tabular}

\section{Hintergrund}

Eine zervikale Lymphknotenmetastasierung tritt bei $10-28 \%$ der Patienten mit einem Ösophaguskarzinom auf, vor allem bei zervikalem oder hoch intrathorakalem Sitz des Tumors [110]. Die Detektion von nicht palpierbaren, zervikalen Lymphknotenmetastasen ist sowohl sonografisch als auch mit der CT möglich [111]. Die B-Bild-Sonografie ist der Computertomografie im Nachweis von zervikalen Lymphknotenmetastasen gleichwertig oder geringfügig überlegen [112-114]. Die ultraschallgestützte Fein- nadelaspirationsbiopsie ist zusätzlich geeignet, den metastatischen Lymphknotenbefall morphologisch zu sichern [115 - 117].

Zum laparoskopischen Ultraschall oder dem intra-operativen Ultraschall der Leber existieren derzeit keine methodisch höherwertigen Studien.

\begin{tabular}{|l|l|l|}
\hline 6.9. & Konsensbasierte Empfehlung & $\begin{array}{l}\text { geprüft } \\
\mathbf{2 0 1 8}\end{array}$ \\
\hline EK & $\begin{array}{l}\text { Die Bestimmung zirkulierender Tumormarker zur Diagnose } \\
\text { oder zur Therapieüberwachung des Ösophaguskarzinoms } \\
\text { soll nicht erfolgen }\end{array}$ \\
\hline $\begin{array}{l}\text { Konsens- } \\
\text { stärke }\end{array}$ & Starker Konsens (100\%) \\
\hline
\end{tabular}

\section{Hintergrund}

Derzeit gibt es keine Leitlinien oder Daten-gestützte Empfehlung zur diagnostischen Anwendung von zirkulierenden Tumormarkern zur Primärdiagnose oder zum Monitoring von Ösophaguskarzinomen.

\begin{tabular}{|c|c|c|}
\hline 6.10. & Konsensbasierte Empfehlung & $\begin{array}{l}\text { geprüft } \\
2018\end{array}$ \\
\hline EK & \multicolumn{2}{|c|}{$\begin{array}{l}\text { Der Röntgen-Breischluck soll nicht zur Diagnosestellung } \\
\text { des Ösophaguskarzinoms eingesetzt werden. }\end{array}$} \\
\hline $\begin{array}{l}\text { Konsens- } \\
\text { stärke }\end{array}$ & \multicolumn{2}{|l|}{ Starker Konsens (96\%) } \\
\hline
\end{tabular}

\begin{tabular}{|l|l|l|}
\hline 6.11. & Konsensbasierte Empfehlung & $\begin{array}{l}\text { geprüft } \\
2018\end{array}$ \\
\hline EK & $\begin{array}{l}\text { Zur Diagnostik von lokalen Tumorkomplikationen (Fisteln) } \\
\text { kann eine Röntgen-Untersuchung mit oralem, wasserlösli- } \\
\text { chem Kontrastmittel durchgeführt werden. }\end{array}$ \\
\hline $\begin{array}{l}\text { Konsens- } \\
\text { stärke }\end{array}$ & Starker Konsens (100\%) \\
\hline
\end{tabular}

\section{Hintergrund}

Auf eine routinemäßige Röntgen-Thorax- oder eine Breischluck-Untersuchung (Röntgenkontrastdarstellung des Ösophagus) soll bei erfolgtem Staging mittels Endoskopie, Endosonografie und Computertomografie verzichtet werden, da keine neuen Informationen gewonnen werden.

Eine Röntgen-Thorax-Untersuchung und eine Röntgenkontrastdarstellung des Ösophagus zur Diagnosestellung eines Ösophaguskarzinoms haben heute medizinhistorische Bedeutung. Vor Einführung der Computertomografie waren beide Verfahren feste Bestandteile der Diagnostik und des Stagings des Ösophaguskarzinoms [118]. Heute bringen diese Untersuchung im Hinblick auf die Diagnosestellung und das TNM-Staging keine ergänzenden Erkenntnisse zur Endoskopie, Computertomografie 
und Endosonografie. Zwei Arbeiten aus den Jahren 2002 und 2006 führen die Röntgenkontrastdarstellung des Ösophagus zwar noch als Bestandteil des publizierten Diagnose-Algorithmus auf, ohne aber eine klinische Notwendigkeit oder Relevanz gegenüber den weiteren Verfahren abzuleiten [119, 120]. Die überwiegende Zahl der Arbeiten zur Diagnosestellung und Staging des Ösophaguskarzinoms erwähnt beide Untersuchungen nicht mehr [121 - 123]. Eine Studie aus 2007 beschreibt bei 569 Patienten die mit 90 \% höhere Sensitivität des Thorax-CT gegenüber einer Röntgenthoraxuntersuchung mit $68 \%$ bei der Entdeckung pulmonaler Metastasen von Ösophagus- und Kardiakarzinomen [114].

Eine weitere Arbeit [124] erwähnt die Röntgenkontrastdarstellung des Ösophagus als Option in der bildgebenden Diagnosestellung von ösophago-trachealen Fisteln, bevorzugt aber auch hier das Thorax-CT. Diese Untersuchung sollte bei V. a. Fistel oder Perforation mit $50 \mathrm{ml}$ wasserlöslichem Kontrastmittel durchgeführt werden.

\subsection{2. Evidenzbasierte Empfehlung geprüft 2018}

Empfeh- Bei Patienten mit einem neu diagnostizierten Ösophaguslungsgrad karzinom sollte zum primären Staging eine MultidetektorB CT (MDCT) von Hals/Thorax und Abdomen (mit multiplanaren Rekonstruktionen und Wanddistension mit oralem negativem Kontrastmittel) und zusätzlich i. v. Kontrastmittel durchgeführt werden.

Level of Literatur: [125-132]

Evidence

4

Konsens- Konsens (89\%)

stärke

\section{Hintergrund}

Da die Prognose des Ösophaguskarzinoms deutlich mit dem TNM-Stadium korreliert, ist ein möglichst exaktes, prätherapeutisches Schnittbild-Staging prognoserelevant und für die Entscheidung der individuellen Therapiestrategie obligat [133 - 136].

Um bei der MDCT in allen Raumebenen rekonstruieren zu können, ist die Minimalanforderung an den Scannertyp ein Multidetektor-CT mit mindestens 16 Zeilen (isotrope Voxel). Üblicherweise werden bei den heutigen Scannergenerationen mindestens 64 Zeilen verwendet, um zu einer Ortsauflösung von unter $1 \mathrm{~mm}$ zu gelangen und damit ein adäquates T-Staging zu ermöglichen. Die CT sollte zur Verbesserung des T-Stagings als Protokolloptimierung stets mit einer Wanddistension [125], vornehmlich negatives, orales KM, idealerweise 1-1,5 I Wasser unter Spasmolyse in folgender Weise (sog. „Hydro-Technik“) durchgeführt werden: Der Patient sollte über einen Zeitraum von etwa 25-40 Minuten vor Untersuchungsbeginn etwa 1 Liter Wasser trinken. Unmittelbar vor Beginn des CT-Scans werden auf dem Scannertisch weitere ca. 150 - $200 \mathrm{ml}$ Wasser verabreicht. Abhängig von der Lokalisation des Tumors kann eine Rücken- oder Bauchlage erwogen werden. Die empfohlene Schichtdicke beträgt dabei 2,5-3 mm.
Zusätzlich zu der Negativ-Kontrastierung mittels Wasser ist eine i.v. Kontrastmittelgabe mit jodhaltigem Kontrastmittel obligat. Neben der vollständigen Abbildung des Ösophagus sollte die Leber in der portalvenösen Phase aufgenommen werden [126]. Gas-bildende Granulate (wie Weinsäuretartrat) können zur maximalen Wanddistension verabreicht werden, meistens ist aber die alleinige, orale Wassergabe ausreichend. Mehrere Studien belegen, dass es nicht notwendig ist, eine pelvine CT durchzuführen [127, 128]. Isolierte Metastasen des kleinen Beckens jenseits des Skelettssystems bei einem Ösophaguskarzinom sind eine Rarität, weshalb aus Gründen der Dosisreduzierung und Kostenersparnis auf eine Becken-CT verzichtet werden kann.

Die Höhenlokalisation und kranio-kaudale Ausdehnung des Karzinoms ist dabei durch koronare und sagittale Reformatierungen der CT heutzutage gut möglich, weswegen auch diese typische Fragestellung an die Röntgenkontrastdarstellung des Ösophagus obsolet ist. Aufgrund der Mehrzeilentechnologie in der Computertomografie sind auch längere Scanstrecken des gesamten Ösophagus vom zervikalen Anteil bis zum ösophago-gastrischen Übergang heute problemlos in wenigen Sekunden möglich. Es bietet sich daher an, den Hals, - sofern noch keine anderweitige z. B. US-Abklärung erfolgt ist - in das Field of View einzuschließen. In diesem Fall ist eine zusätzliche Ultraschallabklärung des Halses nicht mehr erforderlich. Liegt hingegen bereits eine CT des Abdomen und Thorax ohne die zervikalen Anteile des Ösophagus vor, sollte ergänzend noch eine Ultraschallabklärung des Halses erfolgen. Unabhängig von der Höhenlokalisation des Karzinoms sollte wegen der Möglichkeit von Fernmetastasen immer eine vollständige Abklärung des gesamten Ösophagus angestrebt werden. Der kraniale Rand des Field of view wird daher durch den Oberkiefer markiert, die Scanstrecke sollte grundsätzlich bis zum Leberunterrand reichen.

T-Staging: Die MDCT ist im T-Staging in den frühen Stadien limitiert. Dennoch können unter Verwendung der „Hydro-Technik“ Sensitivitäten von $95 \%$ und ein positiver prädiktiver Wert von $96 \%$ erreicht werden [129]. Ein T-Staging konnte in dieser Studie bei bis zu $76 \%$ der Fälle korrekt durchgeführt werden.

N-Staging: Im LK-Staging ist die EUS sensitiver (68\%) als die CT (33\%), aber weniger spezifisch (58\% vs. $75 \%$ ), - außer wenn sie mit einer Feinnadelpunktion (FNP) kombiniert wird, wie oben unter Punkt 6.6 ausgeführt [130]. In Kombination mit der EUS wird eine Sensitivität von einem nodalem Befall von $91 \%$ erreicht. Eine Kombination von PET-CT, MDCT und EUS erzielt die höchste Genauigkeit für die Ermittlung des LK-Status [53] ( Tab. 7). Die Sensitivität für ein korrektes N-Staging wird dabei unterschiedlich limitiert angegeben, z. B. mit 42 \% für die EUS, 49 \% für die PET und $35 \%$ für das CT [131]. Die Spezifität betrug hierbei $91 \%, 87 \%$ und $93 \%$, die Korrektheit $66 \%$, $68 \%$ und $63 \%$. Es ergaben sich keine statistisch signifikanten Unterschiede. Weitere Ergebnisse siehe $>$ Tab. 7.

M-Staging: Die CT hat eine deutliche Streuung in der Genauigkeit der Detektion einer metastasierten Situation (Sensitivität zwischen 37 - 46\%, Spezifität zwischen 63-80\%). Die multiphasische CT der Leber erhöht die Sensitivität für die Detektion von Leber-Metastasen. Jedoch können hämatogene oder peritoneale Fernmetastasen der CT Diagnostik entgehen, weshalb hier nur Sensitivitäten von $46-81 \%$ und Spezifitäten von $63-82 \%$ erreicht werden [132]. 


\section{MRT zum Staging des Ösophaguskarzinoms}

In Fällen, wenn eine CT nicht durchgeführt werden kann (z. B. bei KM-Kontraindikationen), - oder als ergänzende Untersuchung zu CT/EUS -, kann eine MRT durchgeführt werden. Das MRT ist vergleichbar genau zum CT für das TNM Staging [137], insbesondere bei Tumoren des gastro-ösophagealen Übergangs [138], aber weniger exakt für pulmonale Läsionen [139]. Es ist in keiner Region dem CT überlegen [140]. Der diagnostische Wert für das T-Staging von Ösophagus-Karzinomen mit der MRT ist im Laufe der letzten Jahre stetig gestiegen. Aktuell ist die verfügbare Anzahl von Studien für eindeutige Schlussfolgerungen noch zu gering.

In naher Zukunft hat die MRT das Potenzial, beim Staging die Tumorabgrenzung und eine Echtzeit-Kontrolle für eine Radiotherapie zu verbessern. Gleiches gilt auch für das Therapieansprechen, insbesondere in der individualisierten Tumortherapie. Darüber hinaus kann die funktionelle MRT-Bildgebung als sogenannte Diffusions-MRT [141] über die reine Morphologie hinaus wertvolle Informationen liefern. Zudem gibt es aktuelle Entwicklungen in der MRT, auch die Ösophaguswand genauer darzustellen und damit die Tiefeninfiltration besser abzubilden - und damit das T-Staging ggf. weiter zu verbessern [142].

\begin{tabular}{|l|l|l|}
\hline $\mathbf{6 . 1 3 .}$ & Evidenzbasierte Empfehlung & $\begin{array}{l}\text { modifi- } \\
\text { ziert2018 }\end{array}$ \\
\hline $\begin{array}{l}\text { Empfeh- } \\
\text { lungsgrad } \\
\mathbf{0}\end{array}$ & $\begin{array}{l}\text { Bei lokal fortgeschrittenen Tumoren (cT 2-4 und cN+) } \\
\text { kann zusätzlich eine PET/CT-Untersuchung zum M-Stag- } \\
\text { ing eingesetzt werden, falls der Patient potenziell kurativ } \\
\text { behandelbar ist bzw. das Ergebnis klinische Konsequen- } \\
\text { zen hat. }\end{array}$ \\
\hline $\begin{array}{l}\text { Level of } \\
\text { Evidence } \\
\mathbf{1 b}\end{array}$ & \begin{tabular}{l} 
Literatur: $[53,94,95,111,131,143-155]$ \\
\hline $\begin{array}{l}\text { Konsens- } \\
\text { stärke }\end{array}$
\end{tabular} & \begin{tabular}{l} 
Starker Konsens (100\%) \\
\hline
\end{tabular} \\
\hline
\end{tabular}

\section{Hintergrund}

Ein diagnostisches CT ist der gegenwärtige Standard zum M-Staging. Die Kombination von PET mit diagnostischem CT hat die höchste Sensitivität zum M-Staging und erfasst üblicherweise den Körperstamm (PET/CT: Schädelbasis bis proximale Femora und diagnostisches CT von Hals, Thorax und Abdomen). Der Gemeinsame Bundesausschuss (G-BA) hat 2014 einen Beschluss zur ambulanten spezialärztlichen Versorgung von Patienten mit schweren Verläufen bei gastrointestinalen Tumoren und Tumoren der Bauchhöhle herausgegeben: darin eingeschlossene Patienten mit Ösophagus-Karzinom können eine PET bzw. PET-CT zur Detektion von Fernmetastasen erhalten, wenn die §116b-Anträge genehmigt werden.

Das Institut für Qualität und Wirtschaftlichkeit im Gesundheitswesen (IQWiG) hat hingegen in seinem Abschlussbericht Nr. 172 zur „Positronen-Emissionstomografie (PET) und PET/CT bei Ösophaguskarzinom“ im Juni 2013 festgestellt [156], dass mit den von ihnen angewendeten Methoden kein patientenrelevanter Nutzen für die PET/CT beim Ösophaguskarzinom festge- stellt werden konnte. In Diskrepanz hierzu steht das praktische Vorgehen in den USA, wonach PET/CT-Untersuchungen bei einer Reihe von soliden Tumoren einschließlich des Ösophaguskarzinoms bezahlt werden (CMS Final Decision on PET in Solid Tumors, JNM 2013 und www.cancerpetregistry.org/pdf/FinalNOPR-PETWebinar-6 -19-2013.pdf) [157]. Die Bewertung der PET/CT beim Ösophaguskarzinom weist erhebliche Unterschiede in der internationalen Literatur auf. Originalarbeiten zeigen beim Vergleich CT versus PET-CT eine verbesserte Sensitivität zur Detektion von Fernmetastasen (PET-CT: $71 \%$, CT: $52 \%$ ) [143, 144].

Der von Trachemontagne in 2009 verfasste Bericht AETMIS [95] trennte in der Analyse PET und PET/CT-Daten. PET-Daten kamen von systematischen Reviews und Metaanalysen. Die Sensitivität der PET für das N-Staging betrug 57\% (95\% KI $43-70 \%$ ), die Spezifität $85 \%$ (95\%KI 76 - 95\%). PET verbesserte gegenüber dem CT die Beurteilung distanter Lymphknoten- und Fernmetastasen. Die Sensitivität lag hier bei $71 \%$ (95\% KI: 62 - 79\%) und die Spezifität bei $93 \%$ (95\% KI: 89 - 97\%). Daten zum PET/CT für das initiale Staging kamen aus nur 2 Studien, von denen eine Studie Patienten einschloss, die eine neoadjuvante Chemotherapie erhalten hatten. Für die Beurteilung einer Lymphknotenmetastasierung und das M1a-Staging zeigte das PET/CT eine Sensitivität zwischen $83 \%$ und $94 \%$, wohingegen die Spezifität einen Wert von $92 \%$ erreichte. Dieser Bericht empfahl die PET-CT, wenn die Patienten nach konventionellem Staging (CT und EUS) als chirurgisch kurabel eingestuft wurden. Weitere Studienergebnisse zur Bestätigung dieses Vorgehens wurden gefordert.

Das italienische Dossier 209-2011 ISSN 1591-223X [144] zum Einsatz der FDG-PET beim Ösophaguskarzinom kommt zur Feststellung, dass der Einsatz der FDG-PET zum M-Staging angemessen ist (Level of Evidence: moderat). In dem darin eingeschlossenen systematischen Review von van Vliet et al. [94] wurde die diagnostische Genauigkeit der PET bei Lymphknotenmetastasen (10 Studien, $\mathrm{n}=424$ Patienten) mit einer niedrigen gepoolten Sensitivität (57\%; $95 \% \mathrm{Kl}$ : $43-70 \%$ ), aber einer moderaten gepoolten Spezifität ( $85 \%$; 95 \% KI 76 - 95 \%) und für Fernmetastasen (9 Studien; $n=475$ Patienten) mit einer niedrigen gepoolten Sensitivität (71\%; $95 \% \mathrm{KI}: 62-79 \%$ ) und einer hohen gepoolten Spezifität (93\%; $95 \%$ KI: $89-97 \%$ ) errechnet.

Die englische Leitlinie von Allum et al. [53] empfiehlt PET in Kombination mit EUS und CT für das initiale Staging aufgrund des verbesserten M-Staging in bis zu $30 \%$ der Fälle mit einer Sensitivität von $69-78 \%$ und einer Spezifität von $82-88 \%$. Der belgische KCE Report [111] empfiehlt die PET/CT zum M-Staging, wenn Patienten mit T2 - $4 \mathrm{~N}+$ Ösophaguskarzinomen für eine kurative Therapie nach dem CT- und EUS-Staging in Betracht kommen (starke Zustimmung, niedriger Level der Evidenz). Die Wahrscheinlichkeit, dass es durch PET/CT bei frühen Tumorstadien (cT1 - 2, N0) zu einem Upstaging kommt, ist gering.

Eine Reihe von Autoren berichteten in Originalarbeiten zum klinischen Wert der FDG-PET bzw. FDG-PET/CT zum Staging des Ösophaguskarzinoms: Choi et al. [131, 145] analysierten PET/CT bei 109 Patienten zum N- und M-Staging und fanden jeweils Sensitivitäten von $49 \%$ und $40 \%$ und Spezifitäten von $87 \%$ und $99 \%$. Da in dieser Studie nur chirurgisch resezierbare Patienten eingeschlossen wurden, ist eine Unterschätzung der Sensitivität zur Detektion von Lymphknotenmetastasen möglich. 
Flamen et al. [146] fanden bei 74 Patienten, von denen 34 Fernmetastasen hatten, mit FDG-PET eine bessere diagnostische Genauigkeit von $82 \%$ bei der Detektion von Fernmetastasen im Vergleich zum konventionellen Staging (EUS + CT) von $64 \%$. [147] fanden mit FDG-PET bei 6/39 Patienten (15\%) zusätzliche Fernmetastasen gegenüber dem konventionellen Staging. Fernmetastasen fanden sich in supraklavikulären Lymphknoten (1 Pt.), der Leber (1 Pt.), dem Skelett (1 Pt.), retroperitonealen/Becken-LK (2 Pt.) und sowohl in supraklavikulären LK und der Leber (1 Pt.). Heeren et.al [148] verglichen FDG-PET mit konventionellem Staging (EUS + CT) und fanden mit PET-CT mehr distante Lymphknotenmetastasen $(17 / 24 \mathrm{Pt} .=71 \%$ vs. $7 / 24 \mathrm{Pt} .=29 \%)$. FDG-PET führte bei 15/74 Patienten (20\%) zu einem Upstaging wegen Fernmetastasen. Noble et al. [149] untersuchten den Einfluss der PET/CT bei 191 Patienten, die nach CT/EUS-Staging als chirurgisch heilbar angesehen wurden. Die Sensitivität der PET/CT lag bei $91 \%$, die Spezifität bei $94 \%$. PET/CT detektierte distante Metastasen bei $16 \%$ der Patienten, die bei $9 \%$ der Patienten bestätigt wurden. Kato et al. [150] analysierten PET/CT bei 50 Patienten und alleiniger PET bei 117 Patienten im Hinblick auf das initiale Lymphknotenstaging. Die Sensitivität und diagnostische Genauigkeit von PET/CT (75,9\% und 78\%) waren signifikant besser als von alleiniger PET (55\% and 70,1\%).

Hsu et al. [151] berichteten bei 45 Patienten über eine niedrigere Sensitivität zur Detektion lokaler (Sensitivität 57,1\%) und distanter (Sensitivität 36,4\%) Lymphknotenmetastasen bei moderater Spezifität (83,3\% und 84\%) im Vergleich zu den pathohistologischen Befunden nach Resektion. Barber et al. [152] untersuchte FDG-PET-CT bei 139 Patienten im Primärstaging und fanden eine Änderung im Staging durch PET-CT bei 56/139 Patienten (40\%) und eine Änderung im Management bei 47/139 Patienten (34\%). Cervino et al. [153] verglichen bei 29 Patienten PET/CT mit konventionellem Staging. PET/CT detektierte distante Lymphknotenmetastasen bei 7 Patienten (59\%) und Fernmetastasen bei 4 Patienten (31\%). Die Arbeit von Cervino et al. ist retrospektiv und CT gehört nicht zum konventionellen Staging. Von den 13 im konventionellen Staging nicht richtig bewerteten Patienten, war die CT bei 8 und PET/CT bei 9 Patienten korrekt, sodass die Differenz zwischen CT und PET/CT bei einem Patienten lag. Die Society of Thoracic Surgeons Guidelines on the Diagnosis and Staging of Patients With Esophageal Cancer [154] empfiehlt das PET-CT als das beste Verfahren zum M-Staging mit einer mittleren Sensitivität von $71 \%$ und einer mittleren Spezifität von $93 \%$.

Findlay et al. [155] führten in einer Kohorten-Studie (Evidenzlevel 1b) bei Patienten ohne eindeutigen Metastasennachweis im herkömmlichen Staging routinemäßig sequentiell eine ${ }^{[18 F]}$ FluoroDeoxyGlucose (FDG)-PET/CT, einen endoskopischen Ultraschall (EUS) und eine diagnostische Laparoskopie bei AEG-Tumoren und distalen Ösophaguskarzinomen durch, welche bis unterhalb des Zwerchfells reichten. Das PET/CT änderte das Vorgehen in $23 \%$, indem in 7,1\% Metastasen bestätigt, in $13 \%$ unerwartete Metastasen und in 2,1\% zusätzliche Pathologien identifiziert und in $0,8 \%$ synchrone Karzinome detektiert wurden. Hinsichtlich der Prädiktion unerwarteter Metastasen war die Analyse auf $n=700$ Patienten mit in der CT nachgewiesenen cM0-Befunden beschränkt. Es fanden sich hier allerdings keine Parameter zur Identifikation von Patienten mit ausreichend geringem Metasta-
sen-Risiko, um das Risiko einer PET/CT nicht zu rechtfertigen. Wenngleich sich keine Metastaseninzidenz im Stadium uT1 fand, war das $95 \%$ Konfidenzintervall weit $(0-6,12 \%)$, was nahelegt, dass - im Gegensatz zur gängigen klinischen Praxis - die PET/CT doch einen Stellenwert bei in der Endosonografie nachgewiesenen uT1-Tumoren hat.

\begin{tabular}{|l|l|l|}
\hline $\begin{array}{l}\text { 6.14. } \\
\text { Empreh- }\end{array}$ & $\begin{array}{l}\text { geprüt } \\
\mathbf{2 0 1 8}\end{array}$ \\
\hline $\begin{array}{l}\text { Emidenzbasierte Empfehlung } \\
\text { B }\end{array}$ & $\begin{array}{l}\text { Eine flexible Bronchoskopie sollte bei lokal fortgeschritte- } \\
\text { nen Tumoren mit Kontakt zum Tracheo-Bronchialsystem } \\
\text { auf Höhe - oder oberhalb - der Karina durchgeführt wer- } \\
\text { den. }\end{array}$ \\
\hline $\begin{array}{l}\text { Level of } \\
\text { Evidence } \\
\mathbf{4}\end{array}$ & \begin{tabular}{l} 
Literatur: $[158-162]$ \\
\hline $\begin{array}{l}\text { Konsens- } \\
\text { stärke }\end{array}$
\end{tabular} & Starker Konsens (96\%) \\
\hline
\end{tabular}

\begin{tabular}{|l|l|l|}
\hline 6.15. & Konsensbasierte Empfehlung & $\begin{array}{l}\text { geprüft } \\
\mathbf{2 0 1 8}\end{array}$ \\
\hline EK & $\begin{array}{l}\text { Zum Staging des Ösophaguskarzinoms sollte keine starre } \\
\text { Endoskopie der oberen Luft- und Speisewege durchge- } \\
\text { führt werden. }\end{array}$ \\
\hline $\begin{array}{l}\text { Konsens- } \\
\text { stärke }\end{array}$ & Konsens (88\%) \\
\hline
\end{tabular}

\section{Hintergrund}

Zu dieser Thematik gibt es bisher relativ wenig Evidenz, da sich nur wenige Studien damit systematisch auseinandergesetzt haben. Frühere Daten weisen auf einen Zusammenhang von Plattenepithel-Ösophaguskarzinomen mit synchron auftretenden Neoplasien im Bronchialbaum/Oropharynx hin, jedoch handelt es sich hierbei in der Regel um Fallserien und Beobachtungsstudien [163]. Beim Patienten mit einem Plattenepithel-Karzinom des Ösophagus kann auf der Grundlage dieser Daten - und der heute üblichen, hohen Sicherheit des Eingriffs -, eine flexible diagnostische Bronchoskopie durchaus erwogen und eingesetzt werden. Die früher übliche „Pan-Endoskopie“ der gesamten erreichbaren Hohlsysteme im Kopf und den Atemwegen hingegen ist aufgrund fehlender Evidenzgrundlage als Routine-Maßnahme zum Staging des Ösophaguskarzinoms unnötig.

Bei klinischem Verdacht auf Vorliegen einer ösophagotrachealen oder -bronchialen Fistel und/oder höhergradiger Infiltration des tracheo-bronchialen Systems kann eine diagnostische Bronchoskopie +/- endobronchialem Ultraschall (EBUS)/Biopsie im Einzelfall klinisch nützlich sein [158, 159]. Die Evidenzgrundlage hierfür ist aber eher gering, da systematische Studien fehlen. Eine neuere, kleinere Studie [160] favorisiert eher den EBUS als die Bronchoskopie zur frühzeitigen Identifizierung von inkurablen Patienten beim Primär-Stanging von Tumoren auf/oberhalb der Karina-Höhe. Ältere Studien [161, 162] geben demgegenüber 
eine Treffsicherheit der Bronchoskopie mit Biopsien/Bürstenzytologie für eine Invasion der Atemwege von bis zu $96 \%$ an, wenn die Tumoren hoch sitzen und lokal weit fortgeschritten sind. Auch die Beweglichkeit des Tracheo-Bronchialsystems bei der Bronchoskopie soll ein Prädiktor für die Infiltration versus reiner Impression durch den Ösophagustumor sein [162].

Auf der Grundlage bisheriger Publikationen mit schwacher Evidenz kann zusammenfassend derzeit eine generelle Bronchoskopie-Staging-Abklärung +/- EBUS-Anwendung nur bei definierten Patienten mit lokal fortgeschrittenen, (supra-) bifurkalen Plattenepithel-Karzinomen empfohlen werden, bei denen die Klinik/ Bildgebung eine mögliche Invasion in das Tracheo-Bronchialsystem suggeriert und die Ergebnisse eine klinische Konsequenz nach sich ziehen würden.

\subsection{Diagnostische Laparoskopie und Thorakoskopie (Staging)}

\begin{tabular}{|l|l|l|}
\hline $\begin{array}{l}\text { 6.16. } \\
\text { modifiziert } \\
\mathbf{2 0 1 8}\end{array}$ & Evidenzbasierte Empfehlung \\
\hline $\begin{array}{l}\text { Empfeh- } \\
\text { lungsgrad }\end{array}$ & $\begin{array}{l}\text { Eine diagnostische Laparoskopie kann bei Adenokarzino- } \\
\text { men des ösophagogastralen Überganges zum Ausschluss } \\
\text { von Metastasen der Leber und/oder des Peritoneums in } \\
\text { fortgeschrittenen Stadien durchgeführt werden (insbe- } \\
\text { sondere im Falle einer cT3-, cT4-Kategorie). }\end{array}$ \\
\hline $\begin{array}{l}\text { Level of } \\
\mathbf{1 b}\end{array}$ & $\begin{array}{l}\text { Literatur: [155] } \\
\text { Konsens- } \\
\text { stärke }\end{array}$ & \begin{tabular}{l} 
Starker Konsens (100\%) \\
\hline
\end{tabular} \\
\hline
\end{tabular}

\section{Hintergrund}

Wenngleich es Hinweise auf einen diagnostischen Gewinn in bestimmten Situationen gibt, ist ein Stellenwert der diagnostischen Laparoskopie und/oder Thorakoskopie im Rahmen des Stagings von Ösophaguskarzinomen und Karzinomen des ösophagogastralen Übergangs nicht eindeutig belegt. Frühe Arbeiten zum Thema aus den 1990er Jahren beinhalteten überwiegend heterogene Patientenkollektive mit Malignomen unterschiedlicher gastrointestinaler Entitäten und konnten in den Subgruppen einzelner Tumorentitäten keine eindeutigen Aussagen treffen. Nieveen van Dijkum et al. [164] fanden beispielsweise 1997 bei $6 \%$ der 64 Patienten mit Karzinomen des Ösophagus oder der Kardia zuvor unbekannte Fernmetastasen. Eine Studie aus 2007 von De Graaf et al. [165] untersuchte 511 Karzinome des Ösophagus, des ösophagogastralen Übergangs und des Magens. Nach bildgebendem (Computertomografie, Endosonografie) Vorliegen einer tumorbezogenen Resektabilität erfolgte eine ergänzende Laparoskopie. Hierbei wurde eine Irresektabilität bei 28/164 (17\%) der Patienten mit Karzinomen des distalen Ösophagus festgestellt, bei Patienten mit Karzinomen des ösophago-gastralen Übergangs in 11/64 (17\%), bei Magenkarzinomen in 36/109 (33\%). Grund für die Irresektabilität waren in $75 \%$ Fernmetastasen, in $20 \%$ ein lokal fortgeschrittener Tumor, in $5 \%$ ein ausgedehnter Lymphknotenbefall.
Bei Karzinomen des mittleren und oberen Ösophagusdrittels zeigte die Laparoskopie bei keinem der 28 Patienten eine Fernmetastasierung. Der diagnostische Gewinn fand sich fast ausschließlich bei Adenokarzinomen. Nach Ausschluss einer Irresektabilität in der diagnostischen Laparoskopie zeigte sich bei weiteren 27/332 (8,1\%) der Patienten bei der Laparotomie zur Resektion eine Irresektabilität [165]. Eine Untersuchung aus 2008 bei 255 Patienten von Nath et al. [166] belegt ebenfalls, dass der diagnostische Gewinn einer Laparoskopie bei Ösophaguskarzinomen und Karzinomen des ösophago-gastralen Übergangs geringer ist als bei Magenkarzinomen (9,2\% vs. $28,8 \%$ ), zeigt aber auch, dass eine Pertitoneal-Lavage bei 7,2\% (15/207) der makroskopisch unauffälligen Laparoskopien den Nachweis von Tumorzellen erbringt mit einem schlechten medianen Überleben von 13 Monaten. Aufgrund der sehr kleinen Fallzahl dieser Subgruppe kann aus dieser Studie jedoch keine allgemeine Empfehlung für eine Therapieentscheidung abgeleitet werden.

Noch begrenzter ist die Studienlage zur diagnostischen Thorakoskopie. 1995 zeigten Krasna et al. [167] bei 49 Patienten, dass eine Thorakoskopie geeignet sein kann, mediastinale Lymphknoten zur erweiterten Diagnostik zu gewinnen. Eine US-amerikanische Multicenterstudie bestätigte diese Aussage [168] und sie fand doppelt so viele positive Lymphknoten durch die Thorakoskopie wie durch die konventionelle, nicht invasive Diagnostik. Aufgrund fehlender Langzeitdaten können aus dieser Studie keine Aussagen zur klinischen Relevanz dieses Ergebnisses abgeleitet werden. Ein Vergleich dieser thorakoskopischer Methoden mit endosonografisch gestützen Feinnadel-Aspirationen aus paraösophagealen Lymphknoten existiert bisher nicht.

Eine Arbeit aus 1999 von Luketich et al. [169] bemerkt, dass kleine Metastasen, die ein PET-CT nicht zeigt, bei der Laparoskopie und Thorakoskopie gesehen werden können.

In der bereits zitierten Kohorten-Studie von Findlay et al. [155], in der Patienten ohne eindeutigen Metastasennachweis im herkömmlichen Staging routinemäßig sequentiell einer ${ }^{[18 \mathrm{~F}]}$ FluoroDeoxyGlucose (FDG)-PET/CT, einem endoskopischen Ultraschall (EUS) und einer diagnostischen Laparoskopie unterzogen wurden (AEG-Tumoren und distale Ösophaguskarzinome, welche bis unterhalb des Zwerchfells reichten), erhielten $n=397$ Patienten eine diagnostische Laparoskopie. Metastasen wurden bei $n=28$ Patienten nachgewiesen (entsprechend 7,1\%). Diese fanden sich bei $n=2(4 \%)$ der $n=54$ distalen Ösophaguskarzinome, die endoskopisch nicht den ösophago-gastralen Übergang überschritten. Es konnte kein Parameter zur Identifikation des Schwellenwerts nachgewiesen werden, bei dem der Benefit der Untersuchung deren Risiken aufwiegt. Die diagnostische Laparoskopie schien gemäß den Autoren dieser Untersuchung bei Patienten mit Ösophaguskarzinomen $\geq$ T2 gerechtfertigt.

Die aktuell überarbeitete S3-Leitlinie zur Diagnostik und Therapie der Adenokarzinome des Magens und des ösophagogastralen Übergangs (https://www.leitlinienprogramm-onkologie.de/leitlinien/magenkarzinom/) äussert sich zur diagnostischen, prätherapeutischen Laparoskopie folgendermaßen: Die Staging-Laparoskopie verbessert die Therapieentscheidungen beim lokal fortgeschrittenen Magenkarzinom (Insbesondere cT3, cT4) und sollte vor Beginn der neoadjuvanten Therapie durchgeführt werden (Empfehlungsgrad B, LoE 1b). Aufgrund der hohen diagnosti- 
schen Genauigkeit verbessert sie die Therapieentscheidungen beim lokal fortgeschrittenen Magenkarzinom.

Dies betrifft auch die Adenokarzinome des ösophagogastralen Übergangs. Im Gegensatz dazu gibt es derzeit keine Evidenz für die routinemäßige Durchführung der diagnostischen Laparoskopie beim distalen Adenokarzinom des Ösophagus (AEG Typ I nach Siewert), da hier - im Vergleich zu den AEG II-III-Tumoren die Inzidenz einer Peritonealkarzinose sehr selten ist [155]. In der Arbeit von Findlay et al. (Zitat: Findlay JM et al., Br J Surg 2015) [155] änderte sich das Management durch die diagnostische Laparoskopie beim AEG Typ I nur in einem vernachlässigbaren Anteil der Fälle, sodass die diagnostische Laparoskopie beim AEG Typ I nicht routinemäßig empfohlen werden kann.

\subsection{Pathologie}

\begin{tabular}{|l|l|l|}
\hline 6.17. & Konsensbasierte Empfehlung & $\begin{array}{l}\text { geprüft } \\
\mathbf{2 0 1 8}\end{array}$ \\
\hline EK & $\begin{array}{l}\text { Dysplasien/intraepitheliale Neoplasien sollen nach der } \\
\text { gültigen WHO-Klassifikation graduiert werden in negativ, } \\
\text { unklar/fraglich, niedriggradige (low grade/LGD) oder } \\
\text { hochgradige (high grade/HGD) Dysplasie. }\end{array}$ \\
\hline $\begin{array}{l}\text { Konsens- } \\
\text { stärke }\end{array}$ & \begin{tabular}{l} 
Starker Konsens (96\%) \\
\hline
\end{tabular} \\
\hline
\end{tabular}

\begin{tabular}{|l|l|l|}
\hline $\mathbf{6 . 1 8 .}$ & Konsensbasierte Empfehlung & $\begin{array}{l}\text { modifiziert } \\
\mathbf{2 0 1 8}\end{array}$ \\
\hline EK & $\begin{array}{l}\text { Bei histologischer Diagnose einer IEN/Dysplasie im } \\
\text { Barrett-Ösophagus soll der Prozess einer kompetenten } \\
\text { (dokumentierten) pathologischen Zweitmeinung im } \\
\text { Sinne eines Vier-Augen-Prinzips durchgeführt werden. } \\
\text { Bei Dissens oder Unsicherheit bezüglich der Dysplasie- } \\
\text { diagnose soll eine externe Begutachtung erfolgen. }\end{array}$ \\
\hline $\begin{array}{l}\text { Konsens- } \\
\text { stärke }\end{array}$ & Konsens (93\%) \\
\hline
\end{tabular}

\section{Hintergrund \\ Plattenepithel}

Intraepitheliale Neoplasien (IEN/Dysplasien) des Plattenepithels im Ösophagus sind unmittelbare Vorläuferläsionen des ösophagealen Plattenepithelkarzinoms. Das Karzinom-Risiko steigt mit dem Schweregrad der IEN/Dysplasie an [170]: bei LGIEN/LGD 2,9\%, bei HGIEN/HGD 28,3\% und beim Carcinoma in situ auf $34,4 \%$. Darüber hinaus lassen sich bei Nachweis einer IEN/Dysplasie nebenbefundlich in ca. $20 \%$ der Fälle bereits Plattenepithelkarzinome nachweisen, die meist unmittelbar im Tumorrandbereich liegen. Dabei ist die Prävalenz Karzinom-assoziierter IEN/Dysplasien bei frühen Tumorstadien höher, wahrscheinlich infolge einer Destruktion bei der Tumorprogression [171].

Nach der gültigen WHO-Klassifikation soll ein zweistufiges Graduierungssystem verwendet werden [172]; das Carcinoma in situ unterscheidet sich bezüglich des biologischen Verhaltens nicht von der HGD/HGIEN und kann daher in der HGD/HGIEN subsummiert werden.

\section{Barrett-Mukosa}

Wie auch in anderen Lokalisationen des GI-Traktes wird die intraepitheliale Neoplasie/IEN bzw. Dysplasie im Barrett-Ösophagus (BÖ) als eindeutig neoplastisches Epithel ohne Anhalt für ein infiltratives Wachstum definiert [172]. Die Klassifizierung der Dysplasie (intraepithelialer Neoplasie) erfolgt als negativ, unklar/fraglich oder positiv (niedrig- oder hochgradig) [172]. Derzeit ist der Nachweis eine Dysplasie der valideste Marker für ein erhöhtes Malignomrisiko beim BÖ. Die WHO-Klassifikation soll daher obligatorisch bei jedem Befund mit Barrett-Mukosa angegeben werden. Obwohl die meisten Patienten mit BÖ, die ein Karzinom entwickeln, einer linearen Progression von der Metaplasie über eine zunächst niedrig- und dann hochgradige Dysplasie folgen, entsteht in einigen Fällen das Karzinom möglicherweise direkt aus der Metaplasie oder einer niedriggradigen Dysplasie (LGD) bzw. intraepitheliale Neoplasie (LGIEN) [173].

Die histologische Dysplasie-Diagnostik im BÖ unterliegt einer nicht unbeträchtlichen Interobserver-Variabilität (Kappa-Werte von 0,28 bis 0,65 ), v. a. am unteren (Regenerat vs. LGD/LGIEN) und am oberen Ende des Spektrums (HGD/HGIEN vs. Adenokarzinom) [174 - 178], sie liegt jedoch bei der Beurteilung von endoskopischen Mukosa-Resektaten deutlich höher als bei Biopsien [179].

Aufgrund dieser ausgeprägten Interobserver-Variabilität wird in der deutschen Leitlinie „Gastroösophageale Refluxkrankkheit/ Barrett Ösophagus“ [45] empfohlen, dass die Diagnose einer „Dysplasie“ durch einen Referenzpathologen zu bestätigen ist, um Fehldiagnosen zu verringern. Dieser diagnostischen Problematik wird auch in den ESGE-Leitlinien („Statement 9: The diagnosis of any degree of dysplasia (including „indefinite for dysplasia“) in BE „requires confirmation by an expert GI pathologist“) [180] und in den ACG-Leitlinien („Statement 19. For BE patients with dysplasia of any grade, review by two pathologists, at least one of whom has specialized expertise in GI pathology, is warranted because of interobserver variability in the interpretation of dysplasia“) [181] Rechnung getragen. Bevor eine endoskopische Resektion durchgeführt wird, empfiehlt die ESGE die zusätzliche Bestätigung durch einen externen Pathologen.

In den USA wird derzeit versucht, eine Differenzierung der IEN in einen „adenomatösen“ bzw. „intestinalen“ (Kennzeichen: Verlust der Kernpolarität/Pseudoschichtung der Kerne; einschließlich Mischtyp ca. 85\%) und einen „gastral-foveolären“ Typ (Kennzeichen: flaches atypisches Epithel; ca. 15\%) vorzunehmen [182, 183], wobei dem gastralen Typ ein höheres Progressionsrisiko zugeschrieben wird. Diese Unterteilung ist aber bislang noch nicht allgemein etabliert [184-186], passt aber zu der teils „intestinalen“, teils „gastralen“ Differenzierung der Karzinome im distalen Ösophagus/des ösophagogastralen Übergangs [187, 188].

Die Biologie der LGD/LGIEN wird kontrovers diskutiert, insbesondere aufgrund der selbst unter erfahrenen GI-Pathologen höheren Interobserver-Variabilität. Eine LDG/LGIEN kann im Verlauf bei bis zu 2/3 der Fälle regredieren, bei etwa 20\% der Fälle persistieren, bei etwa 13-15\% der Fälle voranschreiten [189]. Aktuelle Daten zum Progressionsrisiko bei LGD/LGIEN zeigen ein 
kumulatives Risiko für eine HGD/HGIEN nach einer LGD/LGIENDiagnose von $85 \%$ in 5 Jahren. Allerdings musste bei $77 \%$ der re-evaluierten Fälle $(n=110)$ die LGD/LGIEN Diagnose revidiert werden [80]. In einer Untersuchung von Gatenby et al. [190] betrug das relative Karzinomrisiko bei einer LGD/LGIEN-Diagnose 2,871 bzw. die jährliche Inzidenz einer HGD/HGIEN und/oder eines Karzinoms 2,2\% (nur Karzinom 1,4\%); jedoch war bei ca. $70 \%$ der Fälle $(n=283)$ im Verlauf keine bzw. keine sichere Dysplasie/IEN mehr nachweisbar.

HGD/HGIEN sind bei bis zu $59 \%$ der Fälle mit einem synchronen oder metachronen Adenokarzinom assoziiert [173, 191, 192]. Die Inzidenz eines Adenokarzinoms in einem Resektat korreliert stark mit dem Vorliegen einer knotigen, ulzerösen oder tumorösen Läsion [193, 194]; Patienten mit flacher, endoskopisch nicht sichtbarer HGD/HGIEN haben ein wesentlich geringeres synchrones Karzinomrisiko [193]. Überdies kommt es darauf an, ob es sich bei der HGD/HGIEN um einen prävalenten oder einen inzidenten Befund handelt (metachrones Adenokarzinom bei $59 \%$ vs. $31 \%$ d. F.) [192].

\begin{tabular}{|c|c|c|}
\hline 6.19. & Konsensbasierte Empfehlung & $\begin{array}{l}\text { geprüft } \\
2018\end{array}$ \\
\hline EK & \multicolumn{2}{|c|}{$\begin{array}{l}\text { Der histopathologische Befund am Biopsiematerial soll die } \\
\text { folgenden Angaben enthalten: } \\
\text { - Art der neoplastischen Läsion (LGD/LGIEN, HGD/ } \\
\text { HGIEN, Karzinom), insbesondere ob ein invasives } \\
\text { Karzinom vorliegt (bei HGD/HGIEN: Klassifikation am } \\
\text { Biopsat als Tis nach UICC) } \\
\text { - Histologischer Typ nach WHO (insbesondere Unter- } \\
\text { scheidung Plattenepithel- versus Adenokarzinom) } \\
\text { - Bei invasiven Adenokarzinomen: Differenzierungsgrad } \\
\text { (Grading) nach aktueller WHO-Klassifikation } \\
\text { - Bei Läsionen im distalen Ösophagus: Ist eine Becherzell- } \\
\text { haltige Barrett-Mukosa vorhanden? }\end{array}$} \\
\hline $\begin{array}{l}\text { Konsens- } \\
\text { stärke }\end{array}$ & Starker Konsens (100\%) & \\
\hline
\end{tabular}

\section{Hintergrund}

Die Mindestanzahl von Biopsien zur sicheren Diagnostik eines Ösophagusmalignoms ist bislang nicht definiert. Aus unserer Sicht gibt es keine evidenzbasierte Empfehlung zur optimalen Anzahl von Zangenbiopsien, die notwendig sind, um möglichst sicher ein Barrett-Karzinom oder Plattenepithelkarzinom zu erfassen und zu diagnostizieren. Nach den Ergebnissen von Harrison und Perry [195] wäre zu postulieren: Je mehr Biopsien, desto wahrscheinlicher ist die Diagnose. Dies ist jedoch problematisch, da eine spätere endoskopische Abtragung nach tiefen/großen Biopsien erschwert sein kann. Daher hat es sich in der Praxis bewährt, mindestens vier mukosale Biopsien aus makroskopisch suspekten Arealen zu entnehmen.

Ein Karzinom im Ösophagus ist definiert als neoplastische Epithelproliferationen, die über die Basalmembran hinaus das Schleimhautstroma (= intramukosales Karzinom) oder die Submukosa und tiefer infiltriert. Aufgrund des unterschiedlichen biologischen Verhaltens ist insbesondere die Unterscheidung zwischen Plattenepithel- und Adenokarzinom klinisch relevant. Bei gering differenzierten oder undifferenzierten (G 3/4) Tumoren soll unter diesem Aspekt eine immunhistologische Phänotypisierung erfolgen, weiterhin auch zur Identifizierung seltener Karzinomtypen, wie dem neuroendokrinen Karzinom sowie der Abgrenzung gegenüber einer sekundären Infiltration (p63, CK5/ 6, CK7, CK8/18, Synaptophysin, Chromogranin, TTF-1 etc.).

\begin{tabular}{|l|l|l|}
\hline 6.20. & Konsensbasierte Empfehlung & $\begin{array}{l}\text { geprüft } \\
\mathbf{2 0 1 8}\end{array}$ \\
\hline EK & $\begin{array}{l}\text { Die histologische Klassifikation und Stadieneinteilung der } \\
\text { Ösophaguskarzinome soll nach der jeweils aktuellen } \\
\text { WHO- und der TNM-Klassifikation der UICC erfolgen. Die } \\
\text { pathologisch-anatomische Begutachtung soll stets voll- } \\
\text { ständig und in standardisierter Form durchgeführt wer- } \\
\text { den. }\end{array}$ \\
\hline $\begin{array}{l}\text { Konsens- } \\
\text { stärke }\end{array}$ & \begin{tabular}{l} 
Starker Konsens (100\%) \\
\hline
\end{tabular} & \\
\hline
\end{tabular}

\section{Hintergrund}

Die aktuell gültige TNM-Klassifikation [196] definiert, dass ein Tumor, dessen Zentrum in einem Abstand von $2 \mathrm{~cm}$ vom ösophagogastralen Übergang liegt und in den Ösophagus hineinreicht, nach der TNM-Klassifikation der Ösophaguskarzinome klassifiziert wird. Tumoren, die den ösophagogastralen Übergang einbeziehen und deren Zentrum innerhalb der proximalen $2 \mathrm{~cm}$ der Cardia liegt (Siewert-Typen I/II) werden ebenfalls nach dem Schema für Ösophaguskarzinome klassifiziert. Tumoren deren Zentren mehr als $2 \mathrm{~cm}$ vom ösophagogastralen Übergang entfernt sind (Siewert Typ III) werden als Magenkarzinome klassifiziert (auch dann, wenn der ösophagogastrale Übergang einbezogen ist).

\section{Anatomische Unterbezirke}

Die Unterteilung der einzelnen Abschnitte des Ösophagus und des Magens erfolgt nach der Einteilung der ICD-O, topografischer Teil [197, 198].

- C15.0: Zervikaler Ösophagus

- C15.3: Oberer thorakaler Abschnitt des intrathorakalen Ösophagus

- C15.4: Mittlerer thorakaler Abschnitt des intrathorakalen Ösophagus

- C15.5: Unterer thorakaler Abschnitt des intrathorakalen Ösophagus

- C16.0: Ösophagogastraler Übergang

\section{Regionäre Lymphknoten}

Unabhängig vom Sitz des Primärtumors sind die regionären Lymphknoten diejenigen, die in dem lymphatischen Abflussgebiet des Ösophagus lokalisiert sind, eingeschlossen die zoeliakalen Lymphknoten und paraösophagealen Lymphknoten des Halses, aber nicht die supraklavikulären Lymphknoten.

Dabei ist zu beachten, dass bei Ösophaguskarzinomen - und besonders bei Karzinomen des ösophagogastralen Übergangs, die in den Magen einwachsen - auch die Lymphknoten des Magens zu den regionären Lymphknoten gerechnet werden [199] ( Tab. 8-10). 
- Tab. 8 Klinische Klassifikation der Ösophaguskarzinome, eingeschlossen Karzinome des ösophagogastralen Übergangs nach der TNM-Klassifikation [196].

\begin{tabular}{|l|l|}
\hline T - Primärtumor \\
\hline TX & Primärtumor kann nicht beurteilt werden \\
\hline T0 & Kein Anhalt für Primärtumor \\
\hline Tis & Carcinoma in situ \\
\hline T1 & $\begin{array}{l}\text { Tumor infiltriert Lamina propria, Muscularis mucosae oder } \\
\text { Submukosa }\end{array}$ \\
\hline - T1a & Tumor infiltriert Lamina propria, Muscularis mucosae \\
\hline - T1b & Tumor infiltriert Submukosa \\
\hline T2 & Tumor infiltriert Muscularis propria \\
\hline T3 & Tumor infiltriert Adventitia \\
\hline T4 & Tumor infiltriert Nachbarstrukturen \\
\hline T4a & $\begin{array}{l}\text { Tumor infiltriert Pleura, Perikard, V. azygos, Zwerchfell oder } \\
\text { Peritoneum }\end{array}$ \\
\hline T4b & $\begin{array}{l}\text { Tumor infiltriert andere Nachbarstrukturen wie Aorta, } \\
\text { Wirbelkörper oder Trachea }\end{array}$ \\
\hline N - Regionäre Lymphknoten \\
\hline NX & Regionäre Lymphknoten können nicht beurteilt werden \\
\hline N0 & Keine regionären Lymphknotenmetastasen \\
\hline N1 & Metastasen in 1 - 2 Lymphknoten \\
\hline N2 & Metastasen in 3 - 6 Lymphknoten \\
\hline N3 & Metastasen in 7 oder mehr regionären Lymphknoten \\
\hline M - Fernmetastasen \\
\hline M0 & Keine Fernmetastasen \\
\hline M1 & Fernmetastasen \\
\hline pTNM: Pathologische Klassifikation (Die pT- und pN-Kategorien \\
\hline entsprechen den T- und N-Kategorien.)
\end{tabular}

Anmerkung: pM0 und pMX sind keine anwendbaren Kategorien.
- Tab. 9 Stadiengruppierung der TNM-Klassifikation der Ösophaguskarzinome, eingeschlossen Karzinome des ösophagogastralen Übergangs [196] - Plattenepithelkarzinome.

\begin{tabular}{|c|c|c|c|}
\hline & T-Kategorie & N-Kategorie & M-Kategorie \\
\hline \multicolumn{4}{|c|}{ Klinisches Stadium - Plattenepithelkarzinome } \\
\hline Stadium 0 & Tis & No & M0 \\
\hline Stadium I & $\mathrm{T} 1$ & N0, N1 & M0 \\
\hline \multirow[t]{2}{*}{ Stadium II } & $\mathrm{T} 2$ & N0, N1 & M0 \\
\hline & T3 & No & M0 \\
\hline \multirow[t]{2}{*}{ Stadium III } & $\mathrm{T} 1, \mathrm{~T} 2$ & N2 & M0 \\
\hline & T3 & N1, N2 & \\
\hline Stadium IVA & T4a, T4b & Jedes $N$ & M0 \\
\hline Stadium IVA & Jedes $\mathrm{T}$ & N3 & M0 \\
\hline Stadium IVB & Jedes $\mathrm{T}$ & Jedes $\mathrm{N}$ & M1 \\
\hline \multicolumn{4}{|c|}{ Pathologisches Stadium - Plattenepithelkarzinome } \\
\hline Stadium 0 & pTis & pNO & M0 \\
\hline Stadium IA & pT1a & pNO & M0 \\
\hline \multirow[t]{2}{*}{ Stadium IB } & pT1b & pNO & M0 \\
\hline & $\mathrm{pT} 2$ & pNO & M0 \\
\hline \multirow[t]{2}{*}{ Stadium II } & PT3 & pNO & M0 \\
\hline & pT1 & pN1 & M0 \\
\hline \multirow[t]{2}{*}{ Stadium IIIA } & PT1 & pN2 & M0 \\
\hline & pT2 & pN1 & Mo \\
\hline \multirow[t]{3}{*}{ Stadium IIIB } & PT2 & pN2 & M0 \\
\hline & PT3 & pN1, pN2 & Mo \\
\hline & pT4a & pN0, pN1 & M0 \\
\hline \multirow[t]{3}{*}{ Stadium IVA } & pT4a & pN2 & M0 \\
\hline & pT4b & Jedes pN & Mo \\
\hline & Jedes $\mathrm{pT}$ & $\mathrm{pN} 3$ & M0 \\
\hline Stadium IVB & Jedes pT & Jedes pN & M1 \\
\hline
\end{tabular}

\begin{tabular}{|l|l|l|}
\hline 6.21. Konsensbasierte Empfehlung & geprüft \\
2018
\end{tabular}

- Maximale Tiefe der Infiltration: pT1a (m1, m2, m3, $\mathrm{m} 4) / \mathrm{pT} 1 \mathrm{~b}(\mathrm{sm} 1, \mathrm{sm} 2, \mathrm{sm} 3)$ plus Infiltrationstiefe in $\mu \mathrm{m}$ (oder höhere pT-Kategorie)

- Lymphgefäß- und/oder Veneninvasion (L0 vs. L1, V0 vs. $\mathrm{V} 1)$

- Zusammenfassende Einschätzung des LK-Metastasierungsrisikos: Low risk vs. High risk-Resektionsränder bzgl. der Neoplasie (bei ER in toto zirkulärer und basaler Resektionsrand bei „piecemeal“-ER basaler Resektionsrand, da hier der zirkuläre Resektionsrand in der Regel histopathologisch als RX gewertet werden muss)

Konsens- Starker Konsens (100\%)
- Größe der neoplastischen Läsion (wenn möglich in 3 Dimensionen) Karzinom vorliegt (bei HGD/HGIEN: Klassifikation am Resektat als pTis nach UICC)

- Bei Karzinomnachweis: Histologischer Typ nach WHO (insbesondere Unterscheidung Plattenepithel- versus Adenokarzinom, andere seltene Typen)

- Bei invasiven Adenokarzinomen: Differenzierungsgrad (Grading) nach aktueller WHO-Klassifikation stärke 
- Tab.10 Stadiengruppierung der TNM-Klassifikation der Ösophaguskarzinome, eingeschlossen Karzinome des ösophagogastralen Übergangs [196] - Adenokarzinome.

\begin{tabular}{|c|c|c|c|}
\hline Stadium & T-Kategorie & $\mathrm{N}$-Kategorie & M-Kategorie \\
\hline \multicolumn{4}{|c|}{ Klinisches Stadium - Adenokarzinome } \\
\hline Stadium 0 & Tis & No & M0 \\
\hline Stadium I & $\mathrm{T} 1$ & No & M0 \\
\hline Stadium IIA & $\mathrm{T} 1$ & N1 & M0 \\
\hline Stadium IIB & $\mathrm{T} 2$ & No & M0 \\
\hline \multirow[t]{3}{*}{ Stadium III } & $\mathrm{T} 1$ & N2 & MO \\
\hline & $\mathrm{T} 2$ & $\mathrm{~N} 1, \mathrm{~N} 2$ & M0 \\
\hline & T3, T4a & N0, N1, N2 & M0 \\
\hline \multirow[t]{2}{*}{ Stadium IVA } & $\mathrm{T} 4 \mathrm{~b}$ & N0, N1, N2 & M0 \\
\hline & Jedes $\mathrm{T}$ & N3 & M0 \\
\hline Stadium IVB & Jedes $T$ & Jedes N & M1 \\
\hline \multicolumn{4}{|c|}{ Pathologisches Stadium - Adenokarzinome } \\
\hline Stadium 0 & pTis & pNO & M0 \\
\hline Stadium IA & pT1a & pNO & M0 \\
\hline Stadium IB & pT1b & pNO & M0 \\
\hline Stadium IIA & pT2 & pNO & M0 \\
\hline Stadium IIB & pT1a, pT1b & pN1 & M0 \\
\hline \multirow[t]{3}{*}{ Stadium IIIA } & $\mathrm{pT} 1$ & $\mathrm{pN} 2$ & Mo \\
\hline & pT2 & pN1, pN2 & M0 \\
\hline & рT3, pт4a & pNO & \\
\hline \multirow[t]{3}{*}{ Stadium IIIB } & pT2 & $\mathrm{pN} 2$ & M0 \\
\hline & pT3 & pN1, pN2 & Mo \\
\hline & pT4a & pN1 & M0 \\
\hline \multirow[t]{3}{*}{ Stadium IVA } & pT4a & $\mathrm{pN} 2$ & Mo \\
\hline & pT4b & Jedes pN & M0 \\
\hline & Jedes pT & $\mathrm{pN} 3$ & M0 \\
\hline Stadium IVB & Jedes pT & Jedes pN & M1 \\
\hline
\end{tabular}

\section{Hintergrund}

\section{Plattenepithelkarzinom}

Bei der histopathologischen Stadieneinteilung von plattenepithelialen Frühkarzinomen des Ösophagus soll insbesondere im Rahmen endoskopischer Tumorresektionen die Infiltrationstiefe innerhalb der Mukosa bzw. Submukosa nach den Vorgaben der Japanese Society for Esophageal Diseases durchgeführt werden (m1-m3 bzw. sm1-sm3), da eine direkte Korrelation zwischen Infiltrationstiefe und dem Risiko einer lokoregionären LK-Metastasierung besteht (bei m1: 0\%, m2: 3,3\%, m3: 12,2\%; sm1: 26,5\%, sm2: $35,8 \%, s m 3: 45,9 \%)$ laut [200]. In einem chirurgischen Kollektiv betrug sie $0 \%$ für Mukosa-Karzinome vs. $50 \%$ bei Submukosa-Infiltration: sm1: $33 \%$, sm3: $78 \%$ [201].

Daher ist die spezifizierte Infiltrationstiefe (und wahrscheinlich auch der Nachweis einer Angioinvasion) der wichtigste Parameter für die klinische Frage, ob eine lokale Tumorresektion ausreicht oder eine onkologische Resektion erforderlich ist. Überdies konnte auch das Grading als unabhängiger Risikofaktor nachgewiesen werden [201].

\section{Barrett-Adenokarzinom}

Da in der Lamina propria mucosae im Ösophagus Lymph- und Blutgefäße vorkommen, können selbst bei auf die Mukosa begrenzten Adenokarzinomen Lymphknotenmetastasen auftreten [202], dies ist hierbei jedoch deutlich seltener der Fall als beim Plattenepithelkarzinom. Auch hier besteht eine direkte Korrelation zur mikroskopischen Infiltrationstiefe, wobei aufgrund der beim Barrett-Ösophagus typischerweise auftretenden Neo-Muscularis mucosae eine 4-stufige Unterteilung der Mukosa vorgenommen wird: pT1a (m1, m2, m3, m4)/pT1b (sm1, sm2, sm3) plus Infiltrationstiefe in $\mu \mathrm{m}$. Allerdings ist die Genauigkeit der histopathologischen Bestimmung der Infiltrationstiefe

in den Schichten m1-m3 und sm1-sm3 aufgrund von Präparatunzulänglichkeiten nicht immer exakt festzulegen.

\begin{tabular}{|c|c|c|}
\hline 6.22 . & Konsensbasierte Empfehlung & $\begin{array}{l}\text { geprüft } \\
2018\end{array}$ \\
\hline EK & \multicolumn{2}{|c|}{$\begin{array}{l}\text { Der histopathologische Befund an Operationsresektaten } \\
\text { soll folgende Angaben enthalten: } \\
\text { - Größe der neoplastischen Läsion (wenn möglich in } \\
3 \text { Dimensionen) } \\
\text { - Lokalisation des Tumorzentrums in Beziehung zum } \\
\text { ösophagogastralen Übergang (ÖGÜ) und Angabe, ob } \\
\text { der Tumor den ÖGÜ kreuzt (wenn möglich) } \\
\text { - Art der neoplastischen Läsion (LGD/LGIEN, HGD/HGIEN, } \\
\text { Karzinom) - insbesondere, ob ein Karzinom vorliegt } \\
\text { (bei HGD/HGIEN: Klassifikation als pTis nach UICC) } \\
\text { - Bei Karzinomnachweis: Histologischer Typ nach aktuel- } \\
\text { ler WHO-Klassifikation (insbesondere Unterscheidung } \\
\text { Plattenepithel- versus Adenokarzinom, andere seltene } \\
\text { Typen) } \\
\text { - Differenzierungsgrad (Grading) } \\
\text { - Maximale Tiefe der Infiltration (pT) } \\
\text { - Lymphgefäß- und/oder Veneninvasion (L0 vs. L1, V0 vs. V1) } \\
\text { - Resektionsränder: oral, aboral und zirkumferenziell: R0 } \\
\text { vs. R1 } \\
\text { - Status der regionären Lymphknoten nach aktueller UICC- } \\
\text { Klassifikation (pN) und Ratio aus Zahl der befallenen und } \\
\text { untersuchten Lymphknoten (.../...Lymphknoten) }\end{array}$} \\
\hline $\begin{array}{l}\text { Konsens- } \\
\text { stärke }\end{array}$ & Starker Konsens (95\%) & \\
\hline
\end{tabular}

6.23 . Evidenzbasierte Empfehlung

modifiziert 2018

Empfeh- Der histopathologische Befund an Resektaten sollte nach lungsgrad B präoperativer Therapie (neoadjuvanter Therapie) zusätzlich Aussagen zum Regressions-Score enthalten.

Level of Literatur: [203]

Evidence

$2 b$

Konsens-

Starker Konsens (100\%)

stärke 


\section{Hintergrund}

Bei den Empfehlungen zur histopathologischen Befundung an Operations-Resektaten kann die Angabe zur Lokalisation des Tumorzentrums in Beziehung zum ösophago- gastralen Übergang (ÖGU) und Angabe, ob der Tumor den ösophagogastralen Übergang kreuzt, schwierig sein und ist deswegen nicht in allen Fällen möglich: der ÖGÜ wird unterschiedlich definiert, die für die pathologische Anatomie einzig interpretierbare Definition ist der Übergang von Plattenepithel zu Zylinderepithel (Definition nach „the American Heritage Medical Dictionary Copyright 2007“ [204]. Gerade diese Grenzlinie ist sehr häufig bei Karzinomen, insbesondere AEG II nach Siewert [205], im pathohistologischen Präparat nicht mehr erkennbar, da sie von Tumorgewebe durchwachsen bzw. ersetzt wird. Alle übrigen Definitionen [200 - 202] betreffen ausschließlich die physiologische Grenze zwischen Ösophagus und Magen, eine Grenze, welche am Operationspräparat nicht mehr feststellbar ist.

Die postoperative Stadieneinteilung nach UICC (PTNM) ist der wichtigste Prognosefaktor für das Ösophaguskarzinom [206, 207]. Mit zunehmender Infiltrationstiefe nimmt das Risiko einer Lymphknotenmetastasierung sowohl beim Plattenepithel- als auch beim Adenokarzinom signifikant zu [201].

Die stadienunabhängige 5-Jahres-Überlebensrate beträgt sowohl beim Plattenepithel- als auch beim Adenokarzinom 10 $20 \%$; im Stadium 0>95\%, im Stadium I 50-80\%, im Stadium IIA $30-40 \%$, im Stadium IIB $10-30 \%$, im Stadium III 10-15\% und im Stadium IV < 5\% [208].

Neben der pN-Kategorie der UICC wurde auch die Ratio aus metastatisch befallenen Lymphknoten und der Gesamtzahl der untersuchten Lymphknoten als prognoserelevanter Faktor vorgeschlagen [209].

Ein weiterer gesicherter Prognosefaktor ist der R-Status bzgl. einer Infiltration des oralen bzw. aboralen Resektionsrandes [210], dagegen wird dies für den zirkumferenziellen Schnittrand noch kontrovers diskutiert [211, 212].

Überdies konnte der Nachweis von Lymphgefäß-/Veneninvasion als unabhängiger Prognosefaktor identifiziert werden [213, 214]. „Molekularpathologische“ Analysen haben bisher keine zusätzliche diagnostische und prädiktive Bedeutung. Molekulare Prognosefaktoren sind bislang nicht etabliert.

Histologische Ermittlung der Tumor-Regression nach neoadjuvanter Therapie

Die Angabe zum Tumorregressions-Score ist wichtig und sie sollte in jedem Befund enthalten sein, sofern eine präoperative Therapie erfolgt ist. Dabei zeigen Patienten mit kompletter Tumorregression einen signifikant besseren Verlauf als Patienten mit Residualtumor. Zur Evaluation des Therapie-Effektes soll daher das gesamte Tumorbett eingebettet und histologisch untersucht werden. Für die Klassifizierung des Tumorregressionsgrades sind verschiedene Systeme publiziert worden, von denen bislang keines allgemein akzeptiert ist.

Die 5-stufige Einteilung nach Mandard [215] ist primär an Plattenepithelkarzinomen etabliert worden und basiert auf der Abschätzung des Anteils residualer Tumorzellen im Verhältnis zum Ausmaß der Fibrose. Dieser Regressionsscore sollte für Plattenepithelkarzinome verwendet werden. Für Adenokarzinome wird der Score nach Becker et al. empfohlen [216 - 218], da dieser
- Tab. 11 Tumorregressions-Score für Adenokarzinome nach Becker et al. [216-219].

\begin{tabular}{|c|c|}
\hline Regressionsgrad & Definition \\
\hline $1 \mathrm{a}$ & komplette Regression \\
\hline $1 b$ & $\begin{array}{l}\text { subtotale Regression }(1-<10 \% \text { Residualtumor/ } \\
\text { Tumorbett) }\end{array}$ \\
\hline 2 & $\begin{array}{l}\text { partielle Regression (10 - 50\% Residualtumor/ } \\
\text { Tumorbett) }\end{array}$ \\
\hline 3 & $\begin{array}{l}\text { geringe/keine Regression (> } 50 \% \text { Residualtumor/ } \\
\text { Tumorbett). }\end{array}$ \\
\hline
\end{tabular}

in der Anwendung einfacher und deswegen besser nachvollziehbar und auch in Deutschland am meisten verbreitet ist. Der Score wurde kürzlich erweitert [219]. Für Studienuntersuchungen kann die Anwendung eines weiteren Scores nützlich sein, zu empfehlen wäre dann der nach Wu et al. [220] ( $\triangleright$ Tab. 11).

Zwei Arbeiten haben sich mit der Reproduzierbarkeit verschiedener Scoring-Systeme beschäftigt [221, 222] und fanden das System von Becker [216] am besten reproduzierbar.

International wird die 3-stufige Einteilung von Wu favorisiert (P0 $=0 \%$ Residualtumor, P1 $=1-50 \%$, Residualtumor, $\mathrm{P} 2=>50 \%$ Residualtumor), da sie eine geringe Interobserver-Variabilität aufweist [220]. Sie basiert auf der Erkenntnis, dass Patienten ohne Residualtumor den signifikant besten Verlauf zeigten, während die Unterschiede zwischen Fällen mit 1 - 50 \% und > 50\% Residualtumor nicht signifikant waren.

Die prognostische Bedeutung einer kompletten histopathologischen Tumor-Regression nach neoadjuvanter Therapie konnte bei Patienten mit Plattenepithel- und Adenokarzinom des Ösophagus in mehreren Studien belegt werden [217, 223 - 226]. Das ypTNM-Stadium war der beste Prädiktor für das Überleben von Patienten mit lokoregionärem Ösophaguskarzinom nach neoadjuvanter Radiochemotherapie [227]. In einer Kohortenstudie erhielten 400 von 584 operierten Patienten mit einem Ösophagus- oder Übergangskarzinom eine neoadjuvante Chemotherapie [203]. Ein Tumordownstaging nach neoadjuvanter Chemotherapie führte zu einem verbesserten Überleben (HR 0,43; $95 \% \mathrm{Cl}$ $0,31-0,59)$ und war der stärkste unabhängige Überlebensprädiktor - stärker als das klinische prätherapeutische Tumorstadium.

\begin{tabular}{|l|l|l|}
\hline $\mathbf{6 . 2 4 .}$ & Konsensbasierte Empfehlung & $\begin{array}{l}\text { geprüft } \\
\mathbf{2 0 1 8}\end{array}$ \\
\hline EK & $\begin{array}{l}\text { Vor dem Einsatz einer palliativen medikamentösen Tumor- } \\
\text { therapie beim Adenokarzinom soll der HER2-Status als po- } \\
\text { sitiver prädiktiver Faktor für eine potenzielle Therapie mit } \\
\text { Trastuzumab bestimmt werden. Die histopathologische } \\
\text { Bestimmung am Tumorgewebe soll qualitätsgesichert } \\
\text { durchgeführt werden. }\end{array}$ \\
\hline Konsens- & Starker Konsens (100\%) \\
\hline stärke & &
\end{tabular}




\section{Hintergrund}

Die Ergebnisse einer randomisierten Phase-III-Studie (ToGAStudie) bei Patienten mit fortgeschrittenen Karzinomen des Magens (82\%) oder des gastro-ösophagealen Übergangs (18\%) zeigen, dass die Addition des HER2-Antikörpers Trastuzumab zur Standard-Chemotherapie bei Patienten mit positivem HER2-Status eine signifikante und klinisch relevante Verbesserung der medianen Überlebenszeit von 11,1 auf 13,8 Monate bewirkt [228]. Im Vorfeld dieser Studie war der HER2-Status bei 3807 Patienten aus Europa, Lateinamerika und Asien bestimmt worden. Der Anteil der HER2-positiven Magenkarzinome - definiert als eine HER2-Genamplifikation, nachgewiesen mittels Fluoreszenz-insitu-Hybridisierung (FISH +) oder immunhistochemisch 3-fach positiver HER2-Expression (IHC 3+) - lag bei $22 \%$ [229].

In der Subgruppe der Patienten, deren Tumoren IHC3 + oder IHC2 +/FISH + waren, war der Einsatz des HER2-Inhibitors Trastuzumab besonders wirksam. Das mediane Überleben stieg mit Trastuzumab in dieser Subgruppe auf 16 Monate (versus 11,8 Monate in der Gruppe ohne Trastuzumab). In den Subgruppen IHCO/FISH + und IHC1 +/FISH + konnte hingegen für Trastuzumab kein Vorteil in Bezug auf die Überlebenszeit beobachtet werden. Diese Korrelation zwischen dem HER2-Status und der Wirksamkeit von Trastuzumab zeigt eine starke Analogie zum Mammakarzinom, wo die HER2-Inhibition als therapeutische Strategie bei HER2-überexprimierenden Tumoren bereits etabliert ist. Die Ergebnisse der ToGAStudie führen dazu, dass die medikamentöse Therapie des metastasierten Magenkarzinoms und Adenokarzinoms des ösophagogastralen Übergangs erstmals aufgrund eines prädiktiven molekularbiologischen Faktors definiert wird. Für eine Indikation zur Therapie mit Trastuzumab wird (gemäß der Richtlinie der EMEA) die HER2Positivität als IHC3 + oder IHC2 +/FISH + definiert. Die Rate der Grad 3 oder 4 unerwünschten Nebenwirkungen unterschieden sich zwischen den beiden behandlungsgruppen (Trastuzumab plus Chemotherapie vs. alleinige Chemotherapie) nicht. Die Chemotherapie bestand dabei aus einer Kombination von Capecetabine/Cisplatin oder 5-Fluouoracil/Cisplatin. [228]. Im Vergleich mit der alleinigen Chemotherapie verbesserte die Kombination von Trastuzumab und Chemotherapie auch die Lebensqualität [230].

\section{Ernährungsmedizinische Versorgung}

\begin{tabular}{|l|l|l|}
\hline 7.1. & Konsensbasierte Empfehlung & $\begin{array}{l}\text { geprüft } \\
\mathbf{2 0 1 8}\end{array}$ \\
\hline EK & $\begin{array}{l}\text { Die ernährungsmedizinische Versorgung von Patienten/ } \\
\text { innen mit Ösophaguskarzinom sollte ein integraler } \\
\text { Bestandteil der onkologischen Diagnostik, Therapie und } \\
\text { Nachsorge sein und eine interdisziplinäre Aufgabe sein. }\end{array}$ \\
\hline $\begin{array}{l}\text { Konsens- } \\
\text { stärke }\end{array}$ & \begin{tabular}{l} 
Starker Konsens (95\%) \\
\hline
\end{tabular} \\
\hline
\end{tabular}

\section{Hintergrund}

Patienten mit Ösophaguskarzinom weisen nicht zuletzt aufgrund einer mechanischen Behinderung der Nahrungspassage sehr frühzeitig einen Gewichtsverlust auf [231].

Hierbei korreliert das Ausmaß der Dysphagie mit dem Ernährungsdefizit [232]. Der Gewichtsverlust kann bereits bei mehr als der Hälfte der Patienten bei der stationären Aufnahme zur Operation über $5 \%$ des gewohnten Körpergewichts und in $40 \%$ über $10 \%$ des gewohnten Körpergewichts betragen. Bei $30 \%$ findet sich ein Serumalbumin < $30 \mathrm{~g} / \mathrm{l}$ [233].

Trotz krankheitsassoziiertem Gewichtsverlust kann der Körpermassenindex (BMI) bei Patienten mit vorbestehendem Übergewicht präoperativ deutlich über der von der World Health Organization (WHO) als kritisch definierten Grenze von $18,5 \mathrm{~kg} / \mathrm{m}^{2}$ liegen. Der Gewichtsverlust für sich bedeutet jedoch eine Veränderung der Körperzusammensetzung, die ein „metabolisches Risiko" nach sich zieht, welches bei Patienten vor und während der Behandlung insbesondere auch bei der Operation berücksichtigt werden muss [234].

Metabolisch handelt es sich um Hochrisikopatienten [235]. Dies gilt umso mehr für den Fall einer palliativen Therapie, wo das Aufhalten eines Gewichtsverlusts die Therapietoleranz erhöhen, Nebenwirkungen und Fatigue reduzieren und die Lebensqualität erhöhen kann (Leitlinie nicht-chirurgische Onkologie). All dies legt eine Erfassung und Beobachtung des Ernährungsstatus, beginnend bei der stationären Aufnahme oder dem ersten Patientenkontakt nahe und erfordert lokale klare Zuständigkeiten mit transparenten klinische Ablaufstandards (SOP) wie für die Indikation zur perkutanen Endoskopischen Gastrostomie (PEG) [236] (siehe auch Empfehlungen 8.14.-8.17., 8.21., 8.39.).

\section{Kurativ intendierte Therapie}

\subsection{Allgemeine Therapieentscheidung}

\begin{tabular}{|l|l|l|}
\hline $\mathbf{8 . 1 .}$ & Konsensbasierte Empfehlung & $\begin{array}{l}\text { geprüft } \\
\mathbf{2 0 1 8}\end{array}$ \\
\hline $\begin{array}{l}\text { Empfeh- } \\
\text { lungsgrad } \\
\text { EK }\end{array}$ & $\begin{array}{l}\text { Therapieempfehlungen sollen in einer interdisziplinären } \\
\text { Tumorkonferenz getroffen werden. Als Grundlage für die } \\
\text { Therapieempfehlung sollen Staging-Informationen, die } \\
\text { Patienten-Komorbiditäten, der Ernährungsstatus und die } \\
\text { Patientenpräferenz berücksichtigt werden. }\end{array}$ \\
\hline $\begin{array}{l}\text { Konsens- } \\
\text { stärke }\end{array}$ & \begin{tabular}{l} 
Starker Konsens (100\%) \\
\hline
\end{tabular}
\end{tabular}

\section{Hintergrund}

Bei der Diagnostik und der Therapie des Ösophaguskarzinoms werden hohe Anforderungen an die beteiligten Fachdisziplinen gestellt. Dies ist einerseits durch die anatomischen Gegebenheiten zu erklären - Nachbarschaft zur Speiseröhre und zum Bronchialsystem und der Lunge - andererseits aber auch durch die Besonderheiten des zu behandelnden Patientenkollektives (insbesondere beim Plattenepithelkarzinom). 
Deshalb ist ein hoher Grad an Interdisziplinarität erforderlich, um die Patienten nach subtiler Diagnostik einer stadiengerechten Therapie zuzuführen. Dies beinhaltet besonders die Entscheidung, welche Patienten alleine durch eine Operation und welche Patienten durch eine Kombination einer neoadjuvanten präoperativen Radiochemotherapie plus Operation oder welche durch eine definitive, alleinige Radiochemotherapie behandelt werden sollten.

Die auszusprechenden Therapieempfehlungen sollen deshalb in einer interdisziplinären Tumorkonferenz unter Einschluss der beteiligten Fachdisziplinen getroffen werden.

\subsection{Endoskopische Therapie}

\subsubsection{Endoskopische Resektion (ER) und lokal ablative Verfahren}

8.2.

alle 4 Teilemp-

fehlungen

\section{Konsensbasierte Empfehlung}

geprüft 2018

- Bei Nachweis einer hochgradigen intraepithelialen Neoplasie oder eines mukosalen Karzinoms (LO, V0, keine Ulzerationen, Grading G1 / G2) im Barrett-Ösophagus soll eine endoskopische Resektion durchgeführt werden, da hierdurch neben der Therapie auch ein Staging der Läsion mit der Frage der Tiefeninfiltration erfolgt.

- Daher ist eine endoskopisch komplette Resektion mit kurativer Intention anzustreben.

- Bei Patienten mit oberflächlicher Submukosainfiltration eines Adenokarzinoms und ohne Risikokriterien ( $\mathrm{pT} 1 \mathrm{sm} 1 ;<500 \mu \mathrm{m}$ Tiefeninvasion, L0, V0, $\mathrm{G} 1 / 2,<20 \mathrm{~mm}$, keine Ulceration) kann die endoskopische Resektion eine ausreichende Alternative zur Operation sein.

- Nach erfolgreicher Resektion von Neoplasien im Barrett-Ösophagus soll die nicht neoplastische Barrett-Mukosa thermisch abladiert werden, um die Rate an metachronen Neoplasien zu senken.

Konsensstärke Starker Konsens (100\%), jeweils für alle 4 Teilempfehlungen

\section{Hintergrund}

Der Begriff endoskopische Resektion (ER) beinhaltet sowohl die endoskopische Mukosaresektion (EMR), die in Saug- und Schneidetechnik durchgeführt wird, sowie die endoskopische Submukosadissektion (ESD). Die EMR wird hierzulande meistens mittels Ligaturset bzw. in Kappentechnik eingesetzt.

Mittlerweile ist die endoskopische Resektion (ER) in Form der EMR in vielen westlichen Ländern als Standardtherapieverfahren für HGIEN und mukosale Adenokarzinome etabliert [111, 237, 238].In zahlreichen Kohortenstudien konnte gezeigt werden, dass die ER sicher und effektiv ist und bei gleicher Kurationsrate eine geringere Morbidität und Mortalität als die Ösophagusresektion aufweist [239-247]. Idealerweise sollte mit der ER die neoplastische Läsion R0-en-bloc entfernt werden, um ein exaktes histologisches Staging zu gewährleisten.
Der Pathologe kann durch sorgfältige Aufarbeitung des Resektats eine genaue Aussage über die Tiefeninfiltration, den Differenzierungsgrad und das mögliche Vorliegen einer Lymphund Veneninvasion treffen. Diese Informationen erlauben eine Risikostratifizierung, sodass entweder die ER die definitive Therapiemaßnahme darstellt oder die Entscheidung zu einer chirurgischen Therapie getroffen werden muss.

Eine Ösophagusresektion sollte immer dann in Erwägung gezogen werden, wenn einer der folgenden Punkte vorliegt:

1. Lymph (L1)- oder Veneninvasion (V1)

2. schlechter Differenzierungsgrad $(\geq \mathrm{G} 3)$

3. tiefe Submukosainfiltration $(\geq 500 \mu \mathrm{m})$

4. Tumorrest am basalen Resektionsrand (R1 basal) [248 - 251]

Im Falle einer nicht sicher kompletten ER oder „piecemeal“-ER einer neoplastischen Läsion mit Nachweis von Tumor am lateralen Resektionsrand (R1 lateral) kann nochmals ein endoskopischer Therapieversuch unternommen werden. Im Rahmen der nächsten Verlaufskontrolle ist eine sorgfältige Evaluation der Resektionsstelle und ggf. Nachresektion bei Vorliegen von Neoplasieresten indiziert [242]. Die Grenze bis zu der neoplastische Läsionen mittels EMR R0-en-bloc reseziert werden können liegt bei ca. 15 mm. Bei größeren neoplastischen Läsionen erfolgt die Resektion in „piecemeal“-Technik. Ein Nachteil der „piecemeal“-EMR ist jedoch die höhere Rezidivrate als bei der Resektion kleinerer Läsionen, die en-bloc reseziert werden können [241].

Zur en bloc-Resektion größerer Läsionen kann neuerdings die endoskopische Submukosadissektion eingesetzt werden. Mit dieser Technik kann eine vom onkologischen Standpunkt her wünschenswerte R0-Resektion unabhängig von der Läsionsgröße erfolgen. Für das Plattenepithelkarzinom konnte in zahlreichen Studien gezeigt werden, dass die ESD der EMR hinsichtlich en-bloc-Resektionsrate, kurativer Resektionsrate und Lokalrezidivrate überlegen ist [252].

Allerdings existieren für das Barrettkarzinom kaum Daten. In einer prospektiven unizentrischen Studie bei 30 Patienten mit HGIEN oder fokalem Barrettkarzinom gelang eine komplette Resektion mit tumorfreien Resektionsrändern trotz ESD in nur 38,5\% der Patienten [253]. Neue Daten aus Japan zeigen, dass durchaus auch beim Barrett-Ösophagus in $85 \%$ der Fälle eine R0-Resektion möglich ist [254]. Offensichtlich hängen die besseren Daten aus Japan damit zusammen, dass die Resektate größer sind und ein höherer Sicherheitsabstand zur Seite gewählt wird. Probst et al zeigten, dass eine ESD beim Barrettadenokarzinom auch hierzulande mit einer en-bloc-Resektionsrate von 95,4\% und einer R0-Resektionsrate von $84 \%$ möglich ist [255].

Bei Patienten mit oberflächlicher Submukosainfiltration eines Adenokarzinoms kann in ausgewählten Fällen die endoskopische Resektion eine ausreichende Alternative zur Operation sein. Manner et al. behandelten 66 Patienten mit low-risk Läsionen (Infiltration sm1, L0, V0, G1/2, keine Ulceration). Eine komplette Remission konnte bei 53 Patienten erzielt werden. Nach einem mittleren follow-up von 47+29,1 Monaten lag die geschätzte 5-Jahres-Überlebesrate bei $84 \%$ [248, 249].

Die Infiltrationstiefe eines Barrettadenokarzinoms ist entscheidend für den Befall von Lymphknoten [256]. Die Rate an Lymphknotenmetastasen war $0 \%$ bei 70 Patienten mit Mucosakarzinom (m1: 
$n=25, m 2: n=10, m 3: n=35)$ und $34 \%$ bei 101 Patienten mit Submukosakarzinom. 30 Patienten mit sm 1 hatten in 13\%, 26 Patienten mit sm 2 in $19 \%$ und 45 Patienten mit sm 3 in $56 \%$ einen Lymphknotenbefall. [257]. Eine Analyse von 472 Patienten zeigte, dass bei einem undifferenzierten oberflächlichen Mukosakarzinom (m1) in $0,8 \%$ ein Lymphknotenbefall vorliegt, bei einem Befall bis in die tiefe Submukosa (sm3) in 41,4\% positive Lymphknoten nachzuweisen sind [258]. Buskens et al konnten mittels EUS in 93 \% das Lymphknotenstadium korrekt voraussagen. Bei m1-m3 sowie sm1 Tumoren fanden sich im EUS keine positiven Lymphknoten, während bei sm2 in $23 \%$ und bei sm3 in $69 \%$ positive Lymphknoten nachweisbar waren [259]. Diese Daten konnten von Westerterp et al. an 120 Patienten, die operiert wurden, bestätigt werden. Von 79 Patienten mit m1-sm1 Tumoren hatte nur 1 Patient (1\%) positive Lymphknoten, während 18 von 44 Patienten (41\%) mit sm2-sm3 Infiltration einen metastasierten Befall der Lymphknoten aufwiesen [260]. Ancona et al. fanden ebenfalls keine positiven Lymphknoten im Stadium m1-sm1 [261]. Eine Literaturzusammenstellung (2005-2014) hat gezeigt, dass bei insgesamt 183 Patienten mit Ösophagektomie wegen sm1-Adenokarzinomen in $12 \%$ der Fälle Lymphknotenmetastasen nachweisbar waren [257, 260, 262 - 267]. Hölscher et al. [257] zeigten, dass nach einer radikalen Ösophagektomie Patienten, die eine sm1 oder sm 2 Infiltration hatten, die gleiche Prognose hatten wie Patienten mit einem Mukosakarzinom und ein signifikant besseres Überleben aufwiesen als Patienten mit sm3 Tumoren.

Ein Problem der EMR von Barrettneoplasien ist die hohe Rate an Rezidiven und metachronen Läsionen, die bis zu 30 \% berichtet wird [268]. Gründe dafür sind, dass bei „piecemeal“-Resektionen per definitionem keine R0-Resektion vorliegt, dass aber auch multifokale Läsionen im Barrett-Ösophagus übersehen werden können. Ein weiteres Problem sind die bestehenden genetischen Alterationen in der Barrettschleimhaut, die durch eine fokale Therapie einer HGIEN oder eines Adenokarzinoms nicht eliminiert werden können und im Verlauf zu metachronen Neoplasien führen.

In einer retrospektiven Analyse konnte die Wiesbadener Arbeitsgruppe zeigen, dass eine Ablation (PDT oder APC) der restlichen, nicht-neoplastischen Barrett-Schleimhaut nach vorheriger Therapie einer HGIEN oder eines mukosalen Karzinoms die Rate an metachronen Neoplasien signifikant senkte [241]. Mittlerweile belegen mehrere Studien, dass ein zweistufiges Konzept bestehend aus initialer ER gefolgt von einer Ablation der nicht-neoplastischen Barrettschleimhaut am effektivsten und komplikationsärmsten ist [246, 247].

Für die Ablation stehen mehrere Verfahren zur Verfügung. Aktuell spielen derzeit nur die Radiofrequenzablation (RFA) und die Argon-Plasma-Coagulation (APC) eine klinische Rolle. Die PDT - lange Jahre als Standardverfahren eingesetzt [269 - 271] - wurde aufgrund der komplexen Handhabung und ihrer Nebenwirkungen (Stenosen und Phototoxizität) nahezu verdrängt und spielt aktuell keine Rolle mehr [272 - 274]. Kürzlich publizierte Langzeitergebnisse zur RFA zeigen, dass es in einer relevanten Anzahl von Patienten zu einem Rezidiv der Barrett-Schleimhaut und einer Neoplasie kommen kann $[275,276]$.

Ein weiteres Ablationsverfahren, das sich etabliert hat, ist die APC-Therapie. Aufgrund der einfachen Handhabung, der hohen Verfügbarkeit und der niedrigen Kosten im Vergleich zur RFA wird die APC-Therapie vor allem zur Ablation des Short-Segment-
Barrett-Ösophagus eingesetzt [277 - 279]. Der Einsatz beim Long-Segment-Barrett-Ösophagus ist sicherlich komplexer und aufwändiger als die RFA. Allerdings existieren bis heute keine prospektiven randomisierten Studien, die beide Methoden miteinander vergleichen.

Zur Kryotherapie gibt es bislang nur spärliche Daten aus den USA [280, 281]. Sie wird deshalb hierzulande nicht eingesetzt.

\begin{tabular}{|l|l|l|}
\hline 8.3. & Konsensbasierte Empfehlung & $\begin{array}{l}\text { geprüt } \\
\mathbf{2 0 1 8}\end{array}$ \\
\hline EK & \begin{tabular}{|l} 
a) Bei Nachweis einer hochgradigen intraepithelialen \\
Neoplasie oder eines mukosalen. Karzinoms (L0, V0, \\
keine Ulzerationen, Grading G1 / G2, Infiltrationstiefe \\
m1/m2) im Plattenepithel sollte eine endoskopische \\
en-bloc-Resektion angestrebt werden, da hierdurch \\
neben der Therapie auch ein Staging der Läsion mit der \\
Frage der Tiefeninfiltration erfolgt. \\
b) Daher ist eine Resektion mit kurativer Intention und \\
R0-Resektion anzustreben.
\end{tabular} \\
\hline Konsens- & Starker Konsens (100\%) \\
\hline stärke & \\
\hline
\end{tabular}

\section{Hintergrund}

In Analogie zum Barrettadenokarzinom stellt die endoskopische Resektion (ER) das Standardverfahren zur Therapie von Mukosakarzinomen im Plattenepithel dar. Erst durch die exakte Beurteilung des Pathologen ist es möglich zu klären, ob eine R0-Resektion bzw. eine low-risk-Situation vorliegt und damit auch, ob die Resektion kurativen Anforderungen genügt.

Anders als beim Barrettadenokarzinom gibt es zur Frage des Resektionsverfahrens zahlreiche Studien aus Japan. Hier hat die ESD klare Vorteile gegenüber der EMR. Insbesondere bei Läsionen $>15 \mathrm{~mm}$ ist die Rate der en-bloc-Resektionen und der kurativen R0-en-bloc-Resektionen signifikant besser. Ishihara et al. hatten bei Läsionen > $15 \mathrm{~mm}$ mit der ESD eine kurative Resektionsrate von 95 \% (20/21 Patienten), während mit der EMR die kurative Resektionsrate mit $52 \%$ (16/31) signifikant schlechter war [282]. Cao et al. kommen in ihrer Metaanalyse ebenfalls zu dem Ergebnis, dass die ESD der EMR hinsichtlich en-bloc-Resektionsrate, R0-en-bloc-Resektionsrate sowie Rezidiven überlegen ist [283].

\begin{tabular}{|l|l|l|}
\hline 8.4. & Konsensbasierte Empfehlung & $\begin{array}{l}\text { geprüft } \\
\mathbf{2 0 1 8}\end{array}$ \\
\hline EK & \begin{tabular}{|l} 
a) Bei Nachweis einer endoskopisch nicht lokalisierbaren, \\
niedriggradigen intraepithelialen Neoplasie im Barrett- \\
Ösophagus, die durch einen Referenzpathologen be- \\
stätigt wurde, sollen Verlaufskontrollen nach 6 Monaten \\
und dann jährlich erfolgen. \\
b) Eine Radiofrequenzablation des gesamten Barrett Seg- \\
ments zur Verhinderung einer Progression der niedrig- \\
gradigen intraepitheliale Neoplasie kann erfolgen. \\
c) Beim Nachweis einer endoskopisch nicht lokalisierbaren \\
hochgradigen intraepithelialen Neoplasie sollte ein \\
ablatives Verfahren zum Einsatz kommen.
\end{tabular} \\
\hline Konsens- & a \& b=Starker Konsens (100\%) c= Konsens (87\%) \\
\hline stärke & \\
\hline
\end{tabular}




\section{Hintergrund}

Wie bereits beschrieben, handelt es sich bei der LGIEN um eine Diagnose mit einer gewissen Progressionsrate [284]. Hvid-Jensen et al. [39] zeigten, dass Patienten mit LGIEN ein 5fach höheres Risiko haben, ein Barrettadenokarzinom zu entwickeln als Patienten ohne LGIEN. Curvers et al. [80] zeigten, dass bei Bestätigung einer LGIEN durch einen Zweitpathologen die Inzidenz für die Entwicklung einer HGIEN oder Karzinoms 13,4\% pro Patient und Jahr beträgt. Wurde die LGIEN nicht bestätigt und zu einem nicht-dysplastischem Barrett-Ösophagus erklärt, lag die Inzidenzrate bei 0,49\%. Wani et al. [40] fanden jedoch kein erhöhtes Progressionsrisiko bei Patienten mit LGIEN im Vergleich zu Patienten ohne LGIEN.

Dennoch werden international Kontrollendoskopien nach 6 12 Monaten empfohlen, bei der alle sichtbaren Läsionen erneut biopsiert werden und zusätzlich Quadrantenbiopsien alle $1-2 \mathrm{~cm}$ entnommen werden. Bei Bestätigung einer LGIEN sind entweder jährlich Kontrollendoskopien oder eine RFA angezeigt.

Die Radiofrequenzablation (RFA) von Barrettepithel mit LGIEN ist sicher und effektiv. In einer prospektiv-randomisierten scheinkontrollierten Studie von Shaheen et al. 2009 [272] konnten mittels RFA $95 \%$ der LGIEN eliminiert werden. Interessanterweise kam es auch in der Schein-Therapie-Gruppe zu einer Elimination in $26 \%$. Phoa et al. [285] untersuchten in einer prospektiv randomisierten Studie die Frage, ob Patienten mit Barrett-Ösophagus und LGIEN überwacht oder mittels RFA behandelt werden sollten. 136 Patienten mit LGIEN wurden 1:1 in den RFA- bzw. Kontrollarm randomisiert. Eine komplette Remission der LGIEN konnte in $98 \%$ der Patienten im Therapiearm erreicht werden. Bei 37\% der Patienten in der Beobachtungsgruppe konnte im Verlauf keine LGIEN mehr gefunden werden. Bezüglich der Progression zeigte sich bereits nach einem medianen Nachbeobachtungszeitraum von 21 Monaten ein hoch signifikanter Unterschied zwischen beiden Gruppen: In der Therapiegruppe kam es bei 1,5\% der Patienten zu einer Progression und in der Beobachtungsgruppe bei $25 \%$. Dieser Unterschied legt nahe, dass die RFA eine gute Alternative zur Verlaufskontrolle nach 6 Monaten zu sein scheint. Allerdings können anhand der Studie keine Aussagen zum Langzeitverlauf getroffen werden.

Ablative Verfahren gelten beim Barrettfrühkarzinom als Therapie der 2. Wahl nach der ER bzw. Operation. Sind jedoch Areale mit HGIEN Veränderungen trotz Wiederholungsuntersuchung an einem Zentrum makroskopisch nicht sichtbar, kann eine gezielte Resektion des Areals nicht erfolgen. In diesen Fällen muss man abwägen, ob ein ablatives Verfahren, eine Resektion des kompletten Barrettareals in „piecemeal“-Technik oder mittels ESD oder ein chirurgisches Verfahren zu wählen ist. In einem derartigen Fall ist das Risiko eine fortgeschrittene Neoplasie zu übersehen sehr gering, sodass eine Untertherapie sehr unwahrscheinlich ist. Hier stellt die RFA eine ausreichende und sichere Therapiemöglichkeit dar [272, 273]. Alternativ kann aber auch die ER insbesondere beim Short-Segment-Barrett-Ösophagus erfolgreich zum Einsatz kommen. Hiermit wäre sowohl eine suffiziente Therapie als auch die histologische Korrelation mit Staging gewährleistet [243, 247].
Der Stellenwert der ablativen Verfahren beim Plattenepithelkarzinom ist von untergeordneter Bedeutung. Die PDT konnte in den 90iger Jahren eine gewisse Bedeutung erlangen, spielt aber heute so gut wie keine Rolle mehr, allenfalls als Salvage-Therapie, wenn andere Verfahren kontraindiziert sind [286].

Die Datenlage zur APC und RFA Therapie ist spärlich. Während die Daten zur RFA eher enttäuschend sind ( 50 \% CR für Dysplasien bei 20 Patienten), schneidet die APC deutlich besser ab (CR von $95 \%$ bei 19 Patienten) [287 - 289]. Die Kryotherapie hat bislang keinen Stellenwert.

\subsubsection{Vorgehen bei Lokalrezidiven nach endoskopischer Therapie}

\begin{tabular}{|l|l|l|}
\hline $\mathbf{8 . 5 .}$ & Konsensbasierte Empfehlung & $\begin{array}{l}\text { geprüft } \\
\mathbf{2 0 1 8}\end{array}$ \\
\hline EK & $\begin{array}{l}\text { Ein auf die Mukosa beschränktes Lokalrezidiv (crT1a cN0 } \\
\text { cM0) nach früherer endoskopischer Resektion eines } \\
\text { mukosalen Karzinoms im Barrett-Ösophagus kann erneut } \\
\text { endoskopisch behandelt werden. } \\
\text { Wenn damit keine R0-Resektion möglich ist, sollte ein } \\
\text { chirurgisches Verfahren gewählt werden. }\end{array}$ \\
\hline $\begin{array}{l}\text { Konsens- } \\
\text { stärke }\end{array}$ & Konsens $(89 \%)$ \\
\hline
\end{tabular}

\section{Hintergrund}

Pech et al. 2014 [242] berichten über 1000 mukosale Barrettadenokarzinome, die endoskopisch behandelt wurden. In 14,5\% kam es im Follow-up von 56,6 \pm 33,4 Monaten zu metachronen bzw. Rezidivkarzinomen. Von diesen 140 Patienten konnten 114 erneut erfolgreich endoskopisch behandelt werden. Dies betrifft besonders die Fälle, in denen die R1 Situation am lateralen Rand besteht. In den Fällen, in denen eine endoskopische Therapie letztendlich versagt, ist eine Operation immer noch unter kurativen Bedingungen möglich. Dies beinhaltet auch die R1-Situation am basalen Resektionsrand [240, 241, 290].

\subsubsection{Nachsorge nach endoskopischer Therapie}

\begin{tabular}{|l|l|l|}
\hline 8.6. & Konsensbasierte Empfehlung & $\begin{array}{l}\text { geprüft } \\
\mathbf{2 0 1 8}\end{array}$ \\
\hline EK & $\begin{array}{l}\text { Nach erfolgreicher endoskopischer Therapie einer hoch- } \\
\text { gradigen intraepithelialen Neoplasie oder eines Frühkar- } \\
\text { zinoms sollen regelmäßige Kontroll-Endoskopien (nach } \\
\text { 3 Monaten, dann für 2 Jahre halbjährlich und danach }\end{array}$ \\
\hline $\begin{array}{l}\text { Konsens- } \\
\text { stärke }\end{array}$ & \begin{tabular}{l} 
Starker Konsens (100\%) \\
\hline
\end{tabular} & \\
\hline
\end{tabular}

\section{Hintergrund}

Das Problem der endoskopischen Therapie von Barrettneoplasien ist das Auftreten von Rezidiven oder metachronen Läsionen, die bis zu 30 \% betragen [239]. Da Rezidive oftmals einer erneuten 
endoskopischen Therapie zugänglich sind, sollten die Kontrollen in den ersten 2 Jahren nach Therapie engmaschig erfolgen. Die Evidenz für dieses Vorgehen ist gering und richtet sich im Wesentlichen nach der in Studien durchgeführten Praxis.

\subsection{Chirurgische Therapie}

\subsubsection{Hospitalvolumen}

\begin{tabular}{|l|l|l|}
\hline 8.7. & Konsensbasierte Empfehlung & $\begin{array}{l}\text { geprüft } \\
2018\end{array}$ \\
\hline EK & $\begin{array}{l}\text { Die operative Therapie von Ösophagustumoren sollte in } \\
\text { Kliniken mit hoher Fallzahl durchgeführt werden. }\end{array}$ \\
\hline $\begin{array}{l}\text { Konsens- } \\
\text { stärke }\end{array}$ & Konsens (94\%) \\
\hline
\end{tabular}

\section{Hintergrund}

In einem systematischen Review mit Meta-Regressionsanalyse konnte gezeigt werden, dass eine Mindestanzahl von 20 durchgeführten komplexen Operationen am Ösophagus pro Jahr nötig ist, um eine deutliche Reduktion der postoperativen Mortalität zu erreichen [291, 292]. Aktuelle Literaturdaten bestätigen diese Mindestanzahl und definieren low-volume Kliniken als solche, die weniger als 20 Ösophagektomien pro Jahr durchführen [293, 294]. Hierbei spielt auch die Fallzahl pro Chirurg eine wesentliche Rolle [295]. Weiterhin gibt es Hinweise, dass sich sowohl „Hospital-Volume“ als auch „Chirurgen-Volume“ auf die Prognose der Patienten mit Ösophaguskarzinom auswirkt und in Kliniken mit hoher Fallzahl ein höheres Langzeitüberleben erreicht wird als in Kliniken mit niedriger Fallzahl [294, 296].

\subsubsection{Präoperative Risikoanalyse}

\begin{tabular}{|l|l|l|}
\hline $\mathbf{8 . 8 .}$ & Konsensbasierte Empfehlung & $\begin{array}{l}\text { geprüft } \\
\mathbf{2 0 1 8}\end{array}$ \\
\hline EK & $\begin{array}{l}\text { Vor geplanter Ösophagektomie soll eine Risikoanalyse } \\
\text { wichtiger Organfunktionen des Patienten erfolgen. Bei } \\
\text { funktioneller Inoperabilität trotz onkologischer Resektabi- } \\
\text { lität sollen andere Therapieverfahren eingesetzt werden. }\end{array}$ \\
\hline $\begin{array}{l}\text { Konsens- } \\
\text { stärke }\end{array}$ & Starker Konsens (100\%) \\
\hline
\end{tabular}

\section{Hintergrund}

Aufgrund des abdomino-thorakalen Zugangs und der notwendigen Einseitenventilation stellt die Ösophagektomie auch bei minimal invasiven Verfahren eine Belastung für den Patienten dar. Daher sollte eine präoperative Überprüfung der funktionellen Belastbarkeit des Patienten erfolgen. Diese sollte die kardiale, respiratorische, hepatische und metabolische Funktion sowie die Kooperationsfähigkeit des Patienten beurteilen [297, 298].

In verschiedenen Studien konnte gezeigt werden, dass bei systematischer Erfassung des Risikos für postoperative Komplika- tionen eine gute Korrelation zur postoperativen Mortalität und Morbidität besteht. Hierzu stehen verschiedene Score-Systeme zur Verfügung, die speziell für die komplexen Ösophaguseingriffe validiert wurden, z. B. Kölner Risikoscore [297], O-POSSUM (angepasster POSSUM Score für Ösophagektomie, [299, 300].

\subsubsection{Chirurgische Technik}

\subsubsection{Ziel der Resektion}

\begin{tabular}{|l|l|l|}
\hline 8.9. & Konsensbasiertes Statement & $\begin{array}{l}\text { geprüft } \\
\mathbf{2 0 1 8}\end{array}$ \\
\hline EK & $\begin{array}{l}\text { Das Ziel der chirurgischen Resektion beim Plattenepithel- } \\
\text { karzinom und Adenokarzinom ist die vollständige Entfer- } \\
\text { nung des Tumors (oral, aboral und in der Zirkumferenz) } \\
\text { und der regionären Lymphknoten. }\end{array}$ \\
\hline $\begin{array}{l}\text { Konsens- } \\
\text { stärke }\end{array}$ & \begin{tabular}{l} 
Starker Konsens (100\%) \\
\hline
\end{tabular} & \\
\hline
\end{tabular}

\subsubsection{Resektionsausmaß}

\begin{tabular}{|c|c|c|}
\hline 8.10. & Konsensbasierte Empfehlung & $\begin{array}{l}\text { modifiziert } \\
2018\end{array}$ \\
\hline EK & \multicolumn{2}{|c|}{$\begin{array}{l}\text { Bei Lokalisation des Tumors } \\
\text { - im ösophagogastralen Übergang (AEG Typ III) sollte } \\
\text { eine totale Gastrektomie mit distaler Ösophagus- } \\
\text { resektion ausgeführt werden. } \\
\text { - im ösophagogastralen Übergang (AEG Typ II) kann } \\
\text { eine totale Gastrektomie mit distaler Ösophagusre- } \\
\text { sektion, eine transthorakale subtotale Ösophagek- } \\
\text { tomie und alternativ eine transhiatale abdomino- } \\
\text { cervicale subtotale Ösophagektomie erfolgen. Eine } \\
\text { ausgedehnte Infiltration der unteren Speiseröhre } \\
\text { favorisiert dabei mehr die Ösophagektomie, eine } \\
\text { ausgedehnte Infiltration des subcardialen Magens } \\
\text { mehr die Gastrektomie. Bei langstreckigem Befall } \\
\text { beider Organe kann eine totale Ösophagogastrek- } \\
\text { tomie erforderlich sein. } \\
\text { - im distalen (incl. AEG Typ I) und mittleren thorakalen } \\
\text { Ösophagus sollte eine transthorakale subtotale } \\
\text { Ösophagektomie durchgeführt werden. } \\
\text { - im oberen thorakalen Ösophagus sollte das Resek- } \\
\text { tionsausmaß zur Wahrung des Sicherheitsabstandes } \\
\text { nach oral ausgedehnt werden. } \\
\text { - im zervikalen Ösophagus soll die Indikation zum } \\
\text { chirurgischen Vorgehen im Vergleich zur definitiven } \\
\text { Radiochemotherapie unter eingehender Nutzen/ } \\
\text { Risikoabwägung diskutiert werden (siehe hierzu auch } \\
\text { Empfehlung 8.32.). Als chirurgisches Verfahren kann } \\
\text { entweder eine totale Ösophagektomie oder in geeig- } \\
\text { neten Fällen eine zervikale Ösophagusresektion über } \\
\text { einen zervikalen Zugang mit oberer Sternotomie } \\
\text { erfolgen. }\end{array}$} \\
\hline $\begin{array}{l}\text { Konsens- } \\
\text { stärke }\end{array}$ & Starker Konsens (96\%) & \\
\hline
\end{tabular}




\subsubsection{Ausmaß der Lymphadenektomie}

\begin{tabular}{|l|l|l|}
\hline $\mathbf{8 . 1 1 .}$ & Konsensbasiertes Statement & $\begin{array}{l}\text { geprüft } \\
\mathbf{2 0 1 8}\end{array}$ \\
\hline EK & $\begin{array}{l}\text { Das Ausmaß der Lymphadenektomie richtet sich nach der } \\
\text { Lokalisation des Primärtumors, wobei drei Felder (abdo- } \\
\text { minal, thorakal und cervikal) unterschieden werden. Die } \\
\text { Zweifeld-Lymphadenektomie stellt den Standard dar. }\end{array}$ \\
\hline $\begin{array}{l}\text { Konsens- } \\
\text { stärke }\end{array}$ & \begin{tabular}{l} 
Starker Konsens (100\%) \\
\hline
\end{tabular} & \\
\hline
\end{tabular}

\subsubsection{Minimal invasive Verfahren (MIC)}

\begin{tabular}{|l|l|l|}
\hline $\mathbf{8 . 1 2 .}$ & Konsensbasiertes Statement & $\begin{array}{l}\text { geprüft } \\
\mathbf{2 0 1 8}\end{array}$ \\
\hline EK & $\begin{array}{l}\text { Sowohl die Ösophagektomie als auch die Rekonstruktion } \\
\text { des Ösophagus können minimal invasiv oder in Kombina- } \\
\text { tion mit offenen Verfahren (Hybrid-Technik) ausgeführt } \\
\text { werden. Der Stellenwert minimal-invasiver Verfahren kann } \\
\text { noch nicht abschließend bewertet werden. }\end{array}$ \\
\hline $\begin{array}{l}\text { Konsens- } \\
\text { stärke }\end{array}$ & \begin{tabular}{l} 
Starker Konsens (100\%) \\
\hline
\end{tabular} \\
\hline
\end{tabular}

\section{Hintergrund \\ Karzinome des mittleren und distalen Ösophagusdrittels inklusive AEG Typ I}

Die chirurgische Resektion stellt eine standardisierte Therapie mit kurativer Intention für alle potenziell resektablen Ösophaguskarzinome im mittleren und distalen Drittel dar. Eine Ausnahme bilden die auf die Mukosa begrenzten Karzinome (T1a N0 M0), wenn sie endoskopisch komplett R0 reseziert werden können [237].

Für die chirurgische Therapie sprechen folgende Argumente:

- Sie bietet die beste lokale Kontrolle des Tumors.

- Die lokoregionale Rezidivfreiheit ist sowohl beim Adeno- als auch beim Plattenepithelkarzinom besser als bei definitiver Radiochemotherapie [301].

- Eine Radiochemotherapie ist nach der Operation beim Auftreten eines Lokalrezidivs möglich.

- Eine Operation nach definitiver Radiochemotherapie ist risikoreich [302, 303].

Das Ziel der kurativen Resektion ist die vollständige Entfernung des Karzinoms nach oral, aboral und in der Zirkumferenz. Um tumorfreie Resektionsränder, d. h. eine R0-Resektion zu erzielen, ist außer beim Mukosakarzinom (T1a MO N0) in der Regel ein proximaler und distaler Sicherheitsabstand von 2-4cm einzuhalten [304].

Das Resektionsausmaß wird bestimmt durch Tumorlokalisation, TNM-Kategorie, Histologie und Ausdehnung des eventuellen Barrett-Ösophagus. Funktionelle Gesichtspunkte des Rekonstruk- tionsorgans (Magen/Colon) sind ebenfalls wichtig für die Festlegung des Resektionsausmaßes. Das Standardverfahren ist die subtotale transthorakale Ösophagektomie mit Resektion des proximalen Magens und Rekonstruktion mit Magenhochzug und hoch intrathorakaler Anastomose [305 - 307]. Aus funktioneller Sicht führt die hoch intrathorakale Anastomose mit weitgehender Transposition des Mageninterponats in den Brustraum zu einem verringerten postoperativen Reflux im Vergleich zu einer tiefer liegenden Anastomose. Dabei wäre der Magen halb im Bauch und halb im Brustkorb, sodass der positive abdominelle Druck den Reflux in Richtung negativem intrathorakalen Druck provoziert.

Mit dieser subtotalen Ösophagektomie und proximalen Magenresektion wird bei Karzinomen des mittleren und distalen Drittels nach oral und aboral ein weiter Sicherheitsabstand zum Tumor erreicht. Ist das Karzinom im oberen thorakalen Ösophagus lokalisiert, so muss das Resektionsausmaß nach oral erweitert werden, evtl. mit zervikaler Anastomose. Bei der en-bloc-Resektion kann auch der notwendige zirkuläre Abstand zur Aorta und zum Tracheobronchialsystem für die angestrebte R0-Resektion eingehalten werden. Für die prinzipielle Entfernung der Vena azygos und des Ductus thoracicus zusammen mit dem Ösophagus gibt es keine sichere Evidenz [308, 309]. Aus Gründen der lokalen Tumorresektion im Gesunden kann dieses aber erforderlich sein. Auch die Ausdehnung der en-bloc-Resektion auf das Pericard oder einen infiltrierten Lungenlappen ist sinnvoll, wenn dadurch eine R0-Resektion erreicht werden kann. T4a Karzinome mit Infiltration der Pleura, des Zwerchfells oder des Pericards gelten als resektabel, während T4b Karzinome mit Infiltration von Aorta, Wirbelkörper oder Trachea als nicht resektabel eingestuft werden [196].

Zur Festlegung des Resektionsausmaßes der Adenokarzinome des ösophagogastralen Übergangs dient die AEG-Klassifikation [310]. AEG Typ I Karzinome, die eindeutig Ösophaguskarzinome sind, werden mit der beschriebenen subtotalen, transthorakalen Ösophagektomie behandelt. Die Resektion des distalen Typ I Adenokarzinoms kann alternativ auch durch eine transhiatale (transmediastinale) stumpfe Ösophagusresektion mit zervikaler Ösophagogastrostomie vorgenommen werden. Dieses Verfahren hat jedoch zwei Nachteile. Erstens ist die Lymphadenektomie weniger radikal [311]. Die prospektiv, randomisierte Studie von Omloo et al. [312] hat die dadurch bedingte signifikant schlechtere Prognose der Patienten gegenüber der transthorakalen Ösophagektomie gezeigt [312]. Dieses ist durch die Studie von Kutup et al. [311] mit nach dem Propensity Score gebildeten Vergleichsgruppen bestätigt worden [311]. Der zweite Nachteil ist, dass bei der transhiatalen Ösophagusresektion immer eine zervikale Anastomose angelegt werden muss. Diese hat eine höhere Insuffizienzrate als die intrathorakale Anastomose. Bougierungspflichtige Strikturen treten dadurch ebenfalls häufiger auf. Bei minimal invasiver Ösophagektomie haben Patienten mit intrathorakaler Ösophagogastrostomie bessere funtionelle Ergebnisse als diejenigen mit cervicaler Anastomose [313].

\section{AEG Typ II und III}

AEG Typ II Karzinome bis $2 \mathrm{~cm}$ unterhalb des ösophagogastralen Übergangs gelten nach der UICC-Klassifikation von 2017 zwar als Ösophaguskarzinome, das Resektionsausmaß richtet sich aber 
nach der genauen Topografie und der Lage der Haupttumormasse. Beim Typ II Karzinom konkurriert die transhiatal erweiterte Gastrektomie plus distaler Ösophagusresektion mit der Ösophagektomie und oberen Magenresektion. Letzteres ist insbesondere dann indiziert, wenn die transhiatal erweiterte Exposition des Ösophagus nicht ausreicht, um eine sichere R0-Resektion zu erreichen oder der intraoperative Schnellschnitt am Ösophagusrand eine Infiltration zeigt. In der prospektiv, randomisierten Studie von Omloo et al. [312] war bei Typ II Karzinomen keine Prognoseverbesserung durch eine transthorakale gegenüber einer transhiatalen Resektion zu verzeichnen [312]. Eine prospektiv randomisierte Studie bei Typ II/III hat gezeigt, dass bei Typ II Karzinomen die Ausdehnung des Resektionsausmaßes nach oral über eine Rippenbogen-Inzision keine Prognoseverbesserung gegenüber der transhiatal erweiterten Resektion erbringt, wenn mit letzterem Verfahren R0 reseziert werden kann [314]. Die inzwischen vorliegenden 10-Jahres Ergebnisse dieser Studie bestätigen die Resultate von 2006 [315]. Die 5- und 10-Jahres-Überlebensraten aller randomisierten Patienten mit Typ II und III Karzinom mit maximal $3 \mathrm{~cm}$ langer Invasion in den distalen Ösophagus und cT2 - 4 Kategorie betrugen $51 \%$ und $37 \%$ für den transhiatalen Zugang und $37 \%$ und $24 \%$ für den Zugang mit Erweiterung durch den linken Rippenbogen. Der log-rank Test zeigte marginale Differenzen zwischen beiden Gruppen: zweiseitig $p=0,060$ und einseitig $p=0,970$ mit einer Hazard-Ratio für links-thorakal versus transhiatal von 1,42 .

Beim Typ III Karzinom war die Prognose der Patienten in dieser Studie sogar nach dem transhiatalen Verfahren besser als nach der Erweiterung über eine Durchtrennung des linken Rippenbogens. Daher ist bei Typ III Karzinomen, die als Magenkarzinome gelten, das Verfahren der Wahl die transhiatal erweiterte Gastrektomie mit distaler Ösophagusresektion [314, 316].

Bei sehr weit fortgeschrittenen Übergangstumoren mit ausgedehnter Infiltration von Ösophagus und Magen kann die totale Ösophago-Gastrektomie mit Rekonstruktion durch Koloninterposition erforderlich sein, um den Tumor zu allen Seiten komplett zu entfernen.

\section{Plattenepithelkarzinome}

Bei Plattenepithelkarzinomen muss zwischen intrathorakalen und zervikalen Karzinomen (s. u.) differenziert werden, insbesondere der Bezug zur Trachea, zur Trachealbifurkation und zu den Hauptbronchien ist von Bedeutung. Diese enge Lagebeziehung erfordert insbesondere bei fortgeschrittenen Tumoren eine exakte Präparationstechnik zur Schonung der Pars membranacea des Tracheobrochialtrakts verbunden mit einer radikalen Ausräumung der Lymphknoten an der Bifurkation und paratracheal. Diese Anforderungen können nur durch die transthorakale Ösophagusdissektion erreicht werden. Eine transhiatale Ösophagusdissektion führt zu einer geringeren Anzahl resezierter Lymphknoten und zu einer signifikant schlechteren Prognose [311].

\section{Lymphadenektomie}

Zweifeld-Lymphadenektomie bedeutet die LymphknotenAusräumung des thorakalen und abdominellen Feldes. Bei der Dreifeld-Lymphadenektomie werden zusätzlich die Lymphknoten des zervikalen Feldes ausgeräumt. Die regionalen Lymphknoten im Oberbauch und im Mediastinum werden bei der Ösophagekto- mie nicht nur direkt peritumoral, sondern auch im entsprechenden Lymphabflussgebiet ausgeräumt [317-320].

Die Entfernung der regionären Lymphknoten, d. h. thorakal und abdominal - D2 und partiell D1 -, evtl. zervikal, stellt den Standard für die operative Behandlung in kurativer Intention dar. Die Entfernung der zervikalen Lymphknoten ist bei Tumoren des mittleren und distalen Ösophagusdrittels nicht ausreichend begründet [319-321]. Der zurzeit akzeptierte Standard ist die sogenannte Zweifeld Lymphadenektomie (LAD), d. h. des thorakalen und abdominalen Kompartments. Bei zervikalen Ösophaguskarzinomen sollte das dritte Feld, d. h. die zervikalen Lymphknoten ebenfalls reseziert werden.

Bei der thorakalen und abdominalen Lymphadenektomie werden in der Regel mehr als 20 regionäre Lymphknoten entfernt und histopathologisch untersucht. Es konnte in mehreren Studien gezeigt werden, dass bei transthorakaler Ösophagektomie und Zweifeld-LAD im Median 27 Lymphknoten entfernt werden, bei der transhiatalen Resektion im Median nur 17 [201, 311]. Bei der transthorakalen subtotalen Ösophagektomie nach Ivor-Lewis mit hoch intrathorakaler Ösophagogastrostomie werden nach einer randomisierten Studie signifikant mehr Lymphknoten (median 22 versus 18) entfernt als bei der transthorakalen Sweet Ösophagektomie mit Anastomose im mittleren Mediastinum [307]. Auch in einer Multicenter-Studie mit mehr als 1500 eingeschlossenen Patienten variierte der Median der entfernten Lymphknoten je nach operativem Zugang zwischen 20 und 33 [318].

In einer weiteren Multicenter-Studie wurde die prognostische Relevanz der Anzahl entfernter Lymphknoten untersucht. Hier wurde bei einem Cut-off value von 23 entfernten Lymphknoten ein signifikanter Überlebensvorteil nachgewiesen [317].

Die Zahl der entfernten und histologisch untersuchten Lymphknoten kann als Folge der neoadjuvanten Radiochemotherapie bei adäquater Lymphadenektomie geringer sein als ohne Vorbehandlung, nach entsprechenden Studien etwa um 2 untersuchte Lymphknoten [322, 323].

Formal ist für die pTNM-Klassifikation des Ösophaguskarzinoms zur Beschreibung von pNO die Entfernung und histologische Untersuchung von mindestens 7 regionären Lymphknoten erforderlich [324]. Für die pTNM-Klassifikation des Magenkarzinoms ist eine Mindestanzahl von 16 Lymphknoten für die pN0-Kategorie notwendig. Da es insbesondere beim Adenokarzinom des ösophagogastralen Übergangs starke Überschneidungen zwischen Magen und Ösophagus gibt, erscheint die Zahl von 16 oder mehr Lymphknoten für diese Festlegung der N-Kategorie deutlich repräsentativer. Die formalen Anforderungen des TNM-Kommittees, die auf einem internationalen Konsens beruhen, repräsentieren Mindestzahlen für die Erstellung der N-Klassifikation. In der chirurgischen Praxis sind die oben genannten Zahlen von 20 30 Lymphknoten realistisch [311, 317]. Daher erscheint als Orientierung die Mindestanzahl von 20 entfernten Lymphknoten bei der Zweifeld-Lymphadenektomie plausibel. Diese Aussage ist als Expertenkonsens zu bewerten.

\section{Limitierte distale Ösophagusresektion}

Beim nicht endoskopisch entfernbarem T1 Mukosakarzinom kann statt der subtotalen Ösophagektomie eine limitierte distale Ösophagusresektion vorgenommen werden. 
Diese kann entweder abdomino-transhiatal oder abdominothorakal mit gesonderter interkostaler oder abdomino-thorakaler Inzision durch den linken Rippenbogen erfolgen. Diese limitierte Resektion sollte wenn möglich Vagus erhaltend, d. h. unter Belassung des vorderen und hinteren Vagusstamms vorgenommen werden, um die Funktion des Restmagens zu erhalten. Zwei Formen der Rekonstruktion sind dabei möglich:

- Die direkte Ösophagogastrostomie im unteren Mediastinum oder

- die Interposition des isoperistaltischen Jejunumsegmentes mit Ösophagojejunostomie im unteren Mediastinum und Jejunogastrostomie im oberen Abdomen.

Bei der direkten Ösophagogastrostomie im unteren Mediastinum ist mit einer hohen Rate an postoperativen Refluxsymptomen zu rechnen (s. o.). Bei Interposition einer isoperistaltischen Jejunumschlinge sind bei Erhaltung der Vagusfunktion die Entleerung des Magens und die Pylorusfunktion normal. Bei Resektion des Nervus vagus muss eine eventuelle Pylorusdilatation oder Pyloroplastik erfolgen [325, 326]. Es konnte aber gezeigt werden, dass die Lebensqualität nach Merendino-Operation nicht besser ist im Vergleich zu Patienten mit subtotaler Ösophagektomie [327].

\section{Spezialfall zervikales Ösophaguskarzinom}

Beim zervikalen Ösophaguskarzinom kann in geeigneten Fällen eine zervikale Ösophagusresektion über einen zervikalen Zugang mit oberer Sternotomie ausgeführt werden, die regionären Lymphknoten werden dabei entfernt. Die Rekonstruktion erfolgt über ein freies Jejunuminterponat mit microvaskulärem Anschluss an venöse und arterielle Halsgefäße. Alternativ kann eine totale Ösophagektomie vorgenommen werden, mit Rekonstruktion durch Magenhochzug oder Koloninterposition.

Die Ösophagusresektionen bis nahe an den oberen Ösophagussphinkter gehen mit einer hohen Rate an postoperativen Anschluckstörungen und einer Aspirationsneigung einher. Weiterhin ist die Rate an Recurrensparesen hoch. Die lange Transpositionsstrecke des Magen- bzw. Koloninterponats führt oft im Spitzenbereich des hochgezogenen Organs zu Vaskularisationsstörungen und damit zur Anastomoseninsuffizienz mit nachfolgenden bougierungspflichtigen Strikturen. Bei freier Dünndarminterposition mit mikrovaskulären Anastomosen sind ähnliche postoperative Probleme zu erwarten, insbesondere bei revisonspflichtigen Thrombosen der anastomosierten Gefäße. Daher ist die definitive Radiochemotherapie von Plattenepithelkarzinomen in dieser Lokalisation ein zunehmend präferiertes Behandlungskonzept. Die Indikation zum chirurgischen Vorgehen bei derart hochsitzenden Tumoren muss aufgrund der vorher genannten Risiken sehr sorgfältig abgewogen und mit dem Patienten unter Nennung der weiteren Therapieoptionen eingehend besprochen werden.

\section{Rekonstruktion}

Nach totaler Gastrektomie mit distaler Ösophagusresektion wird die Kontinuität am häufigsten mit einer Roux-Y Jejunumschlinge und End-zu-Seit Ösophagojejunostomie transhiatal im unteren Mediastinum wiederhergestellt.

Das Verfahren der Wahl zur Rekonstruktion nach subtotaler Ösophagektomie ist der Magenhochzug. Als Verfahren der zweiten Wahl kommt die Koloninterposition in Betracht, insbesondere bei Patienten nach Voroperationen am Magen. In Ausnahmefäl- len, bei sehr günstiger Gefäßanatomie, kann das Jejunum als Interponat eingesetzt werden. In der Regel ist der zu gewinnende Gefäßstiel eines Jejunuminterponats jedoch zu kurz. Die beste Route zur Rekonstruktion ist das hintere Mediastinum. Bei besonderen Bedingungen, z. B. hohes Risiko für ein Rezidiv im hinteren Mediastinum oder geplanter Bestrahlung des hinteren Mediastinums, oder beim zweizeitigen Verfahren mit verzögerter Rekonstruktion kann das vordere Mediastinum retrosternal zum Hochführen des Interponates verwendet werden.

Die Rekonstruktionsroute durch das vordere Mediastinum ist ca. $5 \mathrm{~cm}$ länger als im hinteren Mediastinum. Es besteht bei der Rekonstruktion im vorderen Mediastinum retrosternal die Tendenz zur Siphonbildung an der oberen Thoraxapertur. Dieses bringt oft eine schlechtere Schluckfunktion mit sich. Die Rekonstruktion ante-sternal subcutan bleibt wenigen Ausnahmefällen vorbehalten.

Das häufigste Verfahren ist die einzeitige Resektion und Rekonstruktion. Bei Patienten mit eingeschränkter funktioneller Belastbarkeit oder fraglicher Vaskularisation des vorbereiteten Mageninterponates kann eine zweizeitige Rekonstruktion sinnvoll sein [306, 328].

\section{Pyloroplastik}

Die Bildung des Mageninterponats kann mit und ohne Pyloroplastik ausgeführt werden. Es gibt jedoch weder in einer Metaanalyse noch in systematischen Reviews eine eindeutige Evidenz für die Durchführung der Pyloroplastik beim Magenhochzug [329, 330].

Die Metaanalyse von Urschel [329] basiert auf 9 randomisierten Studien und zeigte für den Vergleich „mit Pyloromyotomie/ Pyloroplastik versus ohne“ keine statistisch signifikanten Unterschiede hinsichtlich:

- operativer Mortalität: 0,92 (95\% KI, 0,34-2,44)

- Anastomoseninsuffizienz: 0,90 (95\% KI, 0,47-1,76)

- pulmonaler Komplikationen: 0,69 (95\% KI, 0,42 - 1,14)

- Komplikationen der Pyloromyotomie/Pyloroplastik: 2,55 (95\% KI, 0,34-18,98)

- fatale pulmonale Aspirationen: 0,25 (95\% KI, 0,04 - 1,60)

Nur bzgl. früh-postoperativer funktioneller Schlauchmagenentleerungsstörungen zeigte die Metaanalyse einen statistisch signifikanten Vorteil der Pylorusdrainage (RR 0,18; 95 \% KI, 0,030,97).

Ein systematischer Review [330] ergab keinen signifikanten Trend pro Pylorusdrainage im Hinblick auf:

- Langzeitergebnisse der Schlauchmagenentleerung,

- Nahrungsmittelaufnahme und des nutritiven Status,

- obstruktive Symptome des oberen Gastrointestinaltrakts

Bezüglich des Langzeitergebnisses „Gallereflux“ bestand ein nicht-signifikanter Trend pro „keine Pylorusdrainage“.

\section{Hiatoplastik}

Wegen der möglichen Ausbildung paragastraler Hiatushernien und der Gefahr der Inkarzeration von Dünndarm oder Kolon sollte nach Magenhochzug oder Koloninterposition bei zu weitem Zwerchfelldurchtritt eine Hiatoplastik vorgenommen werden [331-333]. 


\section{Minimal invasive Ösophagektomie (MIC)}

Die Ösophagusresektion und Rekonstruktion mit Magenhochzug kann minimal invasiv erfolgen. Folgende Varianten sind möglich:

- Total minimal invasiv mit laparoskopischer Gastrolyse und thorakoskopischer Ösophagektomie, entweder mit intrathorakaler oder zervikaler Ösophagogastrostomie.

- Hybridverfahren:

- laparoskopische Gastrolyse und transthorakale offene Ösophagektomie, entweder mit intrathorakaler oder zervikaler Anastomose

- offene Gastrolyse und thorakoskopische Ösophagektomie, entweder mit intrathorakaler oder zervikaler Anastomose.

- laparoskopische Gastrolyse und transhiatale Ösophagektomie mit zervikaler Ösophagogastrostomie

Es gibt ein publiziertes Studienprotokoll eines laufenden prospektiv randomisierten Projektes und eine abgeschlossene prospektiv randomisierte Studie zum Vergleich minimalinvasiver versus offener Verfahren [334, 335]. Die randomisierte Multicenter-Studie (TIME-trial, Netherlands Trial Register, NTR TC 2,452] mit relativ kleinen Patientenzahlen in den beiden Gruppen beinhaltete sowohl intrathorakale als auch cervicale Anastomosen und unterschiedliche Techniken der seitengetrennten Lungenbelüftung in den Vergleichsgruppen [335]. Folgende Ergebnisse wurden erzielt:

- signifikant geringere Rate an pulmonalen Komplikationen

- kürzerer Intensivstationsaufenthalt

- kürzere Verweildauer.

Die 1-Jahres und 3-Jahres Resultate des TIME-trial zur Lebensqualität und Prognose sind inzwischen publiziert worden [336, 337]. Die 1-Jahres-Lebensqualität hinsichtlich physischer Aktivität, globaler Gesundheit und Schmerzen war in der MIC-Gruppe signifikant besser [336]. Auffallend ist jedoch, dass in beiden Gruppen $44 \%$ nach MIC und $39 \%$ nach offener Operation wegen symptomatischen Anastomosen-Stenosen endoskopisch z. T. mehrfach gedehnt werden mussten. Damit hatte fast die Hälfte der Patienten nach der Operation wieder das gleiche Symptom wie vorher: Dysphagie. Dieses sehr ungünstige Ergebnis wirft Fragen auf zur angewandten Operationstechnik.

Das 3-Jahres-Langzeit-Überleben war in den beiden Gruppen des TIME-trial ohne signifikanten Unterschied [337]. Langzeitergebnisse nicht randomisierter Studien zur MIC-Ösophagektomie lassen keine genaue Beurteilung der Prognose im Vergleich zu offenen Verfahren zu [338 - 340].

Der sog. MIRO-Trial - bislang allerdings nur als Abstract publiziert - [341] mit dem Vergleich offene Ösophagektomie und Magenhochzug versus laparoskopische Gastrolyse und Ösophagektomie per Thorakotomie (Hybrid-Verfahren) ergab signifikant geringere Raten bezüglich:

- postoperativer Morbidität,

- postoperativer pulmonaler Komplikationen und

- des postoperativen DINDO-Scores II-IV zugunsten der HybridTechnik
Der einzige Vergleich zwischen der genannten Hybrid-Technik und der total MIC ist von Bonavina beschrieben [342]. In einem propensity-score matching bezüglich Alter, Geschlecht, BodyMass-Index (BMI), forciertes Expirationsvolumen in 1 Sekunde (FEV1), Charlson-Comorbidity Index (CCI), American Society of Anesthesiology (ASA) Score, Histologie, Tumorlokalisation, pTNM-Stadium und neoadjuvante Therapie gab es zwischen beiden Gruppen keine signifikanten Unterschiede von postoperativer Morbidität/Mortalität, Zahl der resezierten Lymphknoten, R0-Resektionsrate und 1-Jahres-Überleben. Nur die Zeitdauer der Operation war bei MIC signifikant länger.

Aufgrund dieser wenigen Daten kann die minimal invasive Chirurgie noch nicht als Standardverfahren empfohlen werden, zumal diese in einzelnen Serien auch erhöhte Komplikationsraten bezüglich Anastomoseninsuffizienzen und Recurrensparesen aufwies [339, 343 - 345].

In retrospektiven Studien zeigte sich auch eine erhöhte Rate an paragastralen Hiatushernien [346, 347], Die Lymphknotenzahl ist in vielen Serien mit minimal invasiver Ösophagektomie geringer als in den Vergleichsgruppen mit offener Chirurgie.

\section{Perioperative Komplikationen}

Aufgrund der hohen Koinzidenz von Begleiterkrankungen und dem häufigen Vorhandensein diverser Risikofaktoren bei Patienten mit Ösophaguskarzinom einerseits und der Invasivität der radikal-chirurgischen Therapie andererseits können perioperative Komplikationen nicht komplett vermieden werden. Die Prävention, die Früherkennung und das entsprechende konsequente Management von Komplikationen nach Ösophagektomie stellen die wichtigsten Ansatzpunkte für die erfolgreiche Behandlung des Ösophaguskarzinoms dar.

Die internationale Esophagectomy Complications Consensus Group (ECCG) hat eine Standardisierung der exakten Dokumentation von Komplikationen bei Ösophagektomie vorgenommen, um Daten aus verschiedenen Zentren vergleichbar zu machen [348]. Die ersten über 2700 dokumentierten Fälle zeigen eine gute Reproduzierbarkeit dieses Dokumentationssystems zur Erfassung von Komplikationen und verläßliche Daten aus großen Zentren [349]. Dieses von Experten erstellte Dokumentationssystem mit genauer Definition und Graduierung von Komplikationen ist zu empfehlen. Es dient der Überprüfung der eigenen Ergebnisse in Morbiditäts- und Mortalitätskonferenzen und der Qualitätssicherung (siehe Kapitel 12. Qualitätsindikatoren).

\subsubsection{Vorgehen bei Oligometastasierung}

\begin{tabular}{|l|l|l|}
\hline $\mathbf{8 . 1 3 .}$ & Konsensbasierte Empfehlung & $\begin{array}{l}\text { geprüft } \\
\mathbf{2 0 1 8}\end{array}$ \\
\hline EK & $\begin{array}{l}\text { Bei präoperativem Nachweis einer Fernmetastasierung } \\
\text { soll keine Operation erfolgen. } \\
\text { Bei intraoperativem Befund vorher nicht bekannter, sehr } \\
\text { limitierter Fernmetastasen können diese zusammen mit } \\
\text { dem Primärtumor entfernt werden. }\end{array}$ \\
\hline $\begin{array}{l}\text { Konsens- } \\
\text { stärke }\end{array}$ & \begin{tabular}{l} 
Starker Konsens (100\%) \\
\hline
\end{tabular} & \\
\hline
\end{tabular}




\section{Hintergrund}

Die Ösophagektomie und gleichzeitige Resektion von Fernmetastasen bringt nach kleinen retrospektiven Serien keinen prognostischen Vorteil. Daher sollte bei präoperativer M1-Situation die Ösophagektomie vermieden werden. Wird jedoch intraoperativ eine kleine gut resektable Metastase z. B. an Lunge oder Leber entdeckt, kann die Metastasenresektion zum einen zur histologischen Sicherung und zum anderen zur vollständigen Tumorresektion im Rahmen der Ösophagektomie ausgeführt werden [350, 351].

\subsubsection{Perioperative Ernährung}

\begin{tabular}{|l|l|l|}
\hline $\mathbf{8 . 1 4}$. & Konsensbasierte Empfehlung & $\begin{array}{l}\text { geprüft } \\
\mathbf{2 0 1 8}\end{array}$ \\
\hline EK & $\begin{array}{l}\text { Im Rahmen der präoperativen Risikostratifizierung soll ein } \\
\text { Screening auf Mangelernährung erfolgen }\end{array}$ \\
\hline $\begin{array}{l}\text { Konsens- } \\
\text { stärke }\end{array}$ & Starker Konsens (100\%) \\
\hline
\end{tabular}

\section{Hintergrund}

Der Einfluss des Ernährungsstatus auf die postoperative Komplikationsrate nach großen Eingriffen ist vielfach gezeigt worden [352, 353]. Trotz krankheitsassoziiertem Gewichtsverlust kann der Körpermassenindex (BMI) bei Patienten mit Übergewicht präoperativ deutlich über der von der World Health Organization (WHO) als kritisch definierten Grenze von $18,5 \mathrm{~kg} / \mathrm{m} 2$ liegen. Der Gewichtsverlust für sich bedeutet eine Veränderung der Körperzusammensetzung, die ein „metabolisches Risiko“ nach sich zieht, welches bei Patienten bei der Planung einer großen Tumoroperation berücksichtigt werden muss [234].

Das krankheitsassoziierte metabolische Risiko kann sehr leicht mit dem „Nutritional Risk Score“ (NRS) [354] erfasst werden. Dieses Screening-Instrument ist auch für chirurgische Patienten in aktuellen Studien validiert worden [355, 356]. In einer großen Kohortenstudie hat sich bei abdominalchirurgischen Patienten die im NRS erfasste verminderte Nahrungsaufnahme in der Woche vor der Krankenhausaufnahme sogar als alleiniger Risikoprädiktor gezeigt [357]). Für ältere chirurgische Patienten (> 65 Jahre) konnten in einer systematischen Übersicht von 15 Studien aus den Jahren 1998 bis 2008 nur der Gewichtsverlust und die mit einem schlechten Ernährungsstatus assoziierte Verminderung des Serumalbumins als prädiktive Parameter der postoperativen Morbidität gefunden werden [234].

Von der Europäischen Gesellschaft für Klinische Ernährung und Stoffwechsel (ESPEN) [352] wird das hohe metabolische Risiko bei Vorliegen eines der folgenden Kriterien definiert:

- Gewichtsverlust $>10$ - 15\% innerhalb von 6 Monaten

- $B M I<18,5 \mathrm{~kg} / \mathrm{m}^{2}$

- SGA Grad C oder NRS>3

- Serumalbumin $<30 \mathrm{~g} / \mathrm{L}$ (bei Ausschluss einer Leber- oder Niereninsuffizienz)

Ein präoperativ niedriger Serum-Albuminspiegel ist ein prognostischer Faktor für postoperative Komplikationen und Letalität, was auch durch neuere Daten bestätigt wird [358, 359].

\begin{tabular}{|l|l|l|}
\hline $\mathbf{8 . 1 5 .}$ & Konsensbasierte Empfehlung & $\begin{array}{l}\text { geprüft } \\
\mathbf{2 0 1 8}\end{array}$ \\
\hline EK & $\begin{array}{l}\text { Unabhängig vom Ernährungsstatus sollte während einer } \\
\text { neoadjuvanten Therapie begleitend eine Ernährungsbe- } \\
\text { ratung angeboten werden. }\end{array}$ \\
\hline $\begin{array}{l}\text { Konsens- } \\
\text { stärke }\end{array}$ & Starker Konsens (95\%) \\
\hline
\end{tabular}

\section{Hintergrund}

Während in Deutschland eine Diätberatung, wenn überhaupt, zumeist postoperativ vor der Entlassung oder während einer Rehabilitationsbehandlung erfolgt, werden in vielen europäischen Ländern die Diätassistentinnen („dietitians“) in der Chirurgie bereits sehr früh präoperativ eingebunden.

So ist ganz aktuell von einer niederländischen Arbeitsgruppe [360] der Einfluss einer intensiven perioperativen Ernährungstherapie (INS) bei Patienten mit Ösophaguskarzinom über ein Jahr prospektiv untersucht worden. Hierbei wurden 37 Patienten (35 mit neoadjuvanter Therapie) in der Interventionsgruppe mit 28 in den drei Jahren zuvor nach Standard, wenn auch mit einem geringeren Anteil neoadjuvant behandelter Patienten verglichen.

Die intensive Ernährungstherapie beinhaltete eine durch eine onkologisch spezialisierte Diätassistentin durchgeführte Beratung mit dem Ziel einer Gewichtserhöhung durch Energieaufnahme von 1,3-1,5-mal dem geschätzten Energiebedarf. Die Patienten wurden zu häufigen Mahlzeiten unter Supplementierung mit Trinknahrung angehalten.

Während der neoadjuvanten Phase bestanden ein-zweiwöchentliche telefonische Kontakte zur Frage von Ernährungsproblemen und zur Gewichtskontrolle. Bei inadäquater oraler Gewichtsaufnahme wurde eine ergänzende Sondenernährung begonnen. Während der Operation erhielten die Patienten eine Feinnadelkatheterjejunostomie, die während des stationären Aufenthalts und auch nach der Entlassung zur Supplementierung bis zum Erreichen einer energiebedarfsdeckenden oralen Nahrungsaufnahme genutzt wurde. Während der stationären Phase wurden die Patienten zweimal wöchentlich von der Diätassistentin visitiert, nach der Entlassung oder während einer adjuvanten Chemooder Radiotherapie alle 1 - 2 Wochen für 3 Monate, danach monatlich bei Bedarf ggf. häufiger bis zum Ende des ersten Jahres. Die Patienten der Kontrollgruppe erhielten nicht regelhaft präoperativ noch eine Diätberatung, jedoch bei der ersten stationären Aufnahme. Intraoperativ wurde auch bei diesen Patienten eine FKJ angelegt. Die Betreuung nach der Entlassung erfolgte nicht strukturiert, sondern vor allem telefonisch.

In der Interventionsgruppe stieg das Körpergewicht zwischen dem ersten Kontakt und der Operation relativ zur Kontrollgruppe um $+4,7 \pm 1,7 \%$ adjustiert für die möglichen Confounder neodadjuvante Vorbehandlung, ASA Score, Rauchen und Alkohol signifikant an $(\mathrm{p}=0,009)$.

Die postoperative Rate schwerer Komplikationen ( $\geq$ IIIb) nach Dindo war signifikant niedriger in der Interventionssgruppe $(9 / 28=32 \%$ vs. $22 / 37=60 \% ; p=0,045)$. Keine Komplikation trat bei $7 / 28=25 \%$ vs. 3/37 = $8 \%$; $p=0,037$ auf. Sowohl die Länge des 
Intensivaufenthalts als auch die Krankenhausverweildauer (25 vs. 19,5 Tage) waren signifikant kürzer $(p=0,039)$. Die Krankenhausletalität $(3 / 28=11 \%$ vs. $1 / 37=3 \%)$ war ohne signifikanten Unterschied. Auch diese Ergebnisse sprechen für eine intensive perioperative ernährungsmedizinische Mitbehandlung gerade bei den großen Tumoroperationen.

Die Indikation zur perkutanen endoskopischen Gastrostomie während einer neoadjuvanten Therapie sollte insbesondere bei geplanter Ösophagusresektion und Magenhochzug äußerst kritisch und nur in Rücksprache mit dem verantwortlichen Chirurgen gestellt werden. Dann ist eine Direktpunktion zur Vermeidung einer Verschleppung von Tumorzellen bei Durchzugstechnik anzustreben [361]. Günstiger ist die Anlage einer Feinnadelkatheterjejunostomie z. B. laparoskopisch, die auch bei der Resektion belassen werden kann.

\begin{tabular}{|l|l|l|}
\hline $\begin{array}{l}\text { 8.16. } \\
\text { Eeprüft }\end{array}$ & $\begin{array}{l}\text { Evidenzbasierte Empfehlung } \\
\mathbf{2 0 1 8}\end{array}$ \\
\hline $\begin{array}{l}\text { Empfeh- } \\
\text { lungsgrad } \\
\text { A }\end{array}$ & $\begin{array}{l}\text { Patienten mit schwerer Mangelernährung d. h. hohem } \\
\text { metabolischen Risiko sollen vor der Operation eine } \\
\text { Ernährungstherapie erhalten, selbst wenn die Operation } \\
\text { verschoben werden muss. }\end{array}$ \\
\hline $\begin{array}{l}\text { Level of } \\
\text { 1a }\end{array}$ & \begin{tabular}{l} 
Literatur: $[18,235,362-367]$ \\
\hline $\begin{array}{l}\text { Konsens- } \\
\text { stärke }\end{array}$
\end{tabular} & Starker Konsens (95\%) \\
\hline
\end{tabular}

\section{Hintergrund}

Hierbei handelt es sich um eine Leitlinienadaptation [235]. Da sehr viele Patienten ihren Energiebedarf präoperativ durch die normale Ernährung nicht decken, sollten diese Patienten unabhängig vom Ernährungsstatus zur Einnahme einer oralen Trinknahrung motiviert werden.

Obwohl die Vorteile einer immunmodulierenden Trinklösung in vielen Studien für Patienten mit Tumoren im Gastrointestinaltrakt gezeigt worden sind, ist die Datenlage für Patienten mit Ösophaguskarzinom nicht eindeutig. Eine Metaanalyse von sechs prospektiven randomisierten kontrollierten Studien mit Einschluss von 628 Patienten mit Operationen von Ösophagus und Magen konnte keine Konsistenz in den klinischen OutcomeParametern bei enteraler Immunonutrition zeigen [362]. So ist auch das geeignete „Timing“ der Immunnutrition prä- oder perioperativ derzeit offen [363].

Die Verschiebung einer Operation mit dem Ziel der ernährungsmedizinischen Konditionierung ist nur bei Patienten mit hohem metabolischem Risiko, d. h. schwerer Mangelernährung gerechtfertigt. Primär sollte immer einer enteralen Ernährung der Vorzug gegeben werden - möglichst prästationär zur Vermeidung einer noskomialen Infektion (Leitlinienadaptation Magenkarzinomm und Klinische Ernährung) [18, 235].

jie et al. [364] haben in einer kontrollierten Studie die Indikation zur präoperativen Ernährung nach dem NRS gestellt. $512 \mathrm{~Pa}$ tienten waren nach dem NRS Risikopatienten (NRS>3). Diese erhielten aufgrund der Erfahrung des Chirurgen ohne Kenntnisse über den NRS enterale oder parenterale Ernährung für sieben Tage präoperativ. Unterschiede der Infektionsrate und der Krankenhausverweildauer wurden bei Patienten mit einem NRS von drei und vier im Fall einer präoperativen Ernährung nicht gefunden.

Von 120 Patienten mit einem NRS von mehr als fünf profitierten diejenigen, welche eine präoperative Ernährung erhielten mit signifikant niedrigerer Komplikationsrate (25,6 vs. $50,6 \%$, $p=0,008)$ und kürzerer Krankenhausverweildauer $(13,7 \pm 7,9$ vs. 17,9 $\pm 11,3$ Tage, $p=0,018$ ).

Durch parenterale Ernährung kann eine Erholung der physiologischen Funktion und des Körpergesamtproteins innerhalb von 7 Tagen erwartet werden. Zu einer weiteren signifikanten Verbesserung kommt es jedoch auch noch in der zweiten Woche [365] Eine aktuelle Cochrane Analyse zur präoperativen parenteralen Ernährung bei Patienten mit gastrointestinalen Operationen zeigte eine signifikante Reduktion der Komplikationen von 45 auf $28 \%$ [366]. Diese Autoren diskutierten einen Bias, da drei der eingeschlossenen Studien mehr als 20 Jahre alt waren. Zwei wichtige Studien [367, 368] mit positivem Ergebnis waren jedoch nicht eingeschlossen wurden. Wenn eine parenterale Ernährung bei Patienten mit einem Gewichtsverlust > 10 \% für 10 Tage präoperativ durchgeführt und postoperativ neun Tage fortgeführt wurde, war die Komplikationsrate signifikant um $30 \%$ niedriger mit Tendenz zur Reduktion der Letalität [367].

\begin{tabular}{|l|l|l|}
\hline 8.17. & Konsensbasierte Empfehlung & $\begin{array}{l}\text { geprüft } \\
\mathbf{2 0 1 8}\end{array}$ \\
\hline EK & $\begin{array}{l}\text { Nach Ösophagusresektion sollte aufgrund des metaboli- } \\
\text { schen Risikos innerhalb von 24h mit einer enteralen } \\
\text { Ernährung begonnen werden. Eine parenterale Supple- } \\
\text { mentierung kann empfohlen werden, wenn weniger als } \\
60-75 \% \text { der Energiemenge auf enteralem Weg zuge- } \\
\text { führt werden können. }\end{array}$ \\
\hline Konsens- & Konsens (86\%) \\
\hline stärke & \\
\hline
\end{tabular}

\section{Hintergrund}

Eine postoperative künstliche Ernährung ist bei Patienten mit Mangelernährung und solchen ohne manifeste Mangelernährung indiziert, wenn vorherzusehen ist, dass der Patient für mehr als sieben Tage unfähig zur oralen Kostzufuhr sein wird. Die Indikation besteht ebenfalls für Patienten, die für mehr als 10 Tage nicht in der Lage sind, mehr als 60 - 75 \% der empfohlenen Energiemenge oral oder enteral aufzunehmen (Leitlinienadaptation Klinische Ernährung Chirurgie) [235]

Die Vorteile einer postoperativ frühzeitigen enteralen Ernährung beginnend innerhalb von 24 Stunden sind in mehreren Metaanalysen im Hinblick auf eine Verminderung der Infektionsrate, der Krankenhausverweildauer und sogar der Letalität gezeigt worden [369 - 371]. Da nach Ösophagusresektion eine frühe orale, am Kalorienbedarf orientierte Ernährung nicht möglich ist, empfiehlt es sich, bereits intraoperativ eine Ernährungssonde entweder duodenal/jejunal zu platzieren oder als Feinnadelkatheterjejunostomie (FKJ) anzulegen [235]. 
Für Patienten nach Ösophagusresektion zeigte eine Beobachtungsstudie signifikante Vorteile der sicheren längerfristigen enteralen Ernährung über die FKJ, insbesondere beim Vorliegen von Anastomosenproblemen [353]. Die Komplikationsrate der FKJ war niedrig (1,5\%) [372]. Für die frühzeitige enterale Ernährung nach Ösophagusresektion und die katheterassoziierten Komplikationen fand sich in einer prospektiven randomisierten kontrollierten Studie kein signifikanter Unterschied zwischen dem Einsatz einer nasoduodenalen Sonde oder einer FKJ [373].

Da nasojejunale und nasoduodenale Sonden signifikant häufiger und früher dislozieren [374, 375] ist die FK] der nasojejunalen oder -duodenalen Sonde zur längerfristigen enteralen Ernährung überlegen [375].

Eine parenterale Supplementierung der enteralen Zufuhr sollte erfolgen, wenn weniger als 60 - 75\% der Energiemenge auf dem enteralen Weg zugeführt werden können (Leitlinienadaptation Klinische Ernährung Chirurgie) [235].

\subsubsection{Vorgehen bei R1 / R2-Resektion}

\begin{tabular}{|c|c|c|}
\hline 8.18. & Konsensbasierte Empfehlung & $\begin{array}{l}\text { geprüft } \\
2018\end{array}$ \\
\hline EK & \multicolumn{2}{|c|}{$\begin{array}{l}\text { Im Falle einer intraoperativ nachgewiesenen R1-Resektion } \\
\text { sollte unabhängig von einer präoperativen Therapie zu- } \\
\text { nächst die Möglichkeit einer kurativen Nachresektion } \\
\text { geprüft werden. Wenn diese nicht möglich ist, sollte nach } \\
\text { Diskussion in der interdisziplinären Tumorkonferenz eine } \\
\text { postoperative Radiochemotherapie erfolgen. } \\
\text { Bei einer postoperativ erkannten R1-Resektion sollte eine } \\
\text { Radiochemotherapie erfolgen, da die Bedingungen für } \\
\text { eine Nachresektion ungünstig sind. In Einzelfällen kann } \\
\text { eine „wait and see“ Strategie empfohlen werden. }\end{array}$} \\
\hline $\begin{array}{l}\text { Konsens- } \\
\text { stärke }\end{array}$ & Starker Konsens (100\%) & \\
\hline
\end{tabular}

\begin{tabular}{|l|l|l|}
\hline $\mathbf{8 . 1 9 .}$ & Konsensbasierte Empfehlung & $\begin{array}{l}\text { geprüft } \\
\mathbf{2 0 1 8}\end{array}$ \\
\hline EK & $\begin{array}{l}\text { Im Falle einer lokoregionären R2-Resektion kann nach } \\
\text { Diskussion in der interdisziplinären Tumorkonferenz eine } \\
\text { postoperative Radiochemotherapie durchgeführt wer- } \\
\text { den. }\end{array}$ \\
\hline $\begin{array}{l}\text { Konsens- } \\
\text { stärke }\end{array}$ & Starker Konsens (100\%) \\
\hline
\end{tabular}

\section{Hintergrund}

Es gilt als gesichert, dass eine inkomplette Entfernung des Primärtumors einen Tumorrückfall nach sich ziehen wird, also als palliativ anzusehen ist. Dies ist für die R2-Resektion unbestritten (makroskopischer Tumorrest verbleibt bei der OP). Jüngere retrospektive Daten aus Großbritannien ziehen jedoch in Zweifel, dass eine R1-Resektion mit mikroskopischem Tumorrest im sog. zirkumferentiellen Resektionsrand unzweifelhaft mit einer schlechten Prognose verbunden ist [376, 377]. Dennoch wird jeder Chirurg ggf. durch Schnellschnittdiagnostik anstreben, eine R0-Resektion nicht nur longitudinal, sondern auch zirkumferentiell zu erzielen.

Falls dies primär nicht gelungen ist, wird bei einer longitudinalen R1-Resektion üblicherweise im Behandlungsteam eine Nachresektion diskutiert, obwohl dies beim Ösophaguskarzinom meist mit einem (zu) großen Aufwand bzw. (zu) hohem Risiko verbunden ist. Darüber hinaus ist das optimale Vorgehen nach einer inkompletten Resektion beim Ösophaguskarzinom nicht gut durch Daten belegt. Prospektive Studien aus den 80iger Jahren legen nahe, dass eine additive Radiotherapie die Lokalrezidivrate, jedoch nicht das rückfallfreie oder gar das Gesamtüberleben verbessern kann [378, 379]. Zur additiven Chemotherapie gibt es

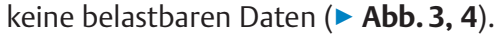

\subsubsection{Vorgehen bei Lokalrezidiv nach Operation}

\begin{tabular}{|l|l|l|}
\hline 8.20. & Konsensbasierte Empfehlung & $\begin{array}{l}\text { geprüft } \\
\mathbf{2 0 1 8}\end{array}$ \\
\hline EK & $\begin{array}{l}\text { Bei einem isolierten Lokalrezidiv nach kurativ intendierter } \\
\text { Operation kann nach Diskussion in einer interdisziplinären } \\
\text { Tumorkonferenz erneut eine Operation durchgeführt wer- } \\
\text { den. Die sorgfältige Evaluation der Operabilität und der } \\
\text { Resektabilität sollte durch ein in der Ösophaguschirurgie } \\
\text { erfahrenes Behandlungsteam vorgenommen werden. } \\
\text { Alternativ soll eine Radiochemotherapie angeboten wer- } \\
\text { den, sofern keine Vorbestrahlung im Rezidivgebiet erfolgt } \\
\text { ist bzw. wenn eine ausreichende Normalgewebetoleranz } \\
\text { vorhanden ist. }\end{array}$ \\
\hline $\begin{array}{l}\text { Konsens- } \\
\text { stärke }\end{array}$ & \begin{tabular}{l} 
Starker Konsens (100\%) \\
\hline
\end{tabular} & \\
\hline
\end{tabular}

\section{Hintergrund}

Prospektive und retrospektive Studien haben gezeigt, dass isolierte Lokalrezidive oder Lymphknotenmetastasen von Plattenepithelkarzinomen des Ösophagus nach Operation ohne eine präoder postoperative Radiochemotherapie mit einer definitiven Strahlentherapie oder Radiochemotherapie im Rezidiv eine kurative Chance haben [380 - 382].

Dabei stellte sich auch in dieser Situation die simultane Radiochemotherapie als effektiver heraus. Die 5-Jahre-Überlebensraten nach Salvage-Radiochemotherapie lagen in den genannten Studien zwischen $14 \%$ und $45 \%$, sie unterscheiden sich also nicht wesentlich von denen in der Primärtherapie. Anastomosenrezidive hatten in zwei der Studien eine schlechtere Prognose als regionale Lymphknotenmetastasen. Mit zunehmender Zahl befallener regionaler Lymphknotenmetastasen im Rezidiv nimmt die Prognose ab. Das Zeitintervall zwischen Primärtherapie und Rezidivtherapie war in keiner der Studien von Bedeutung.

Bei isolierten Lokalrezidiven eines Adenokarzinoms des Ösophagus kommt ebenfalls eine Radiochemotherapie in Frage. Langzeitüberlebende wurden auch hier beobachtet [383, 384]. Teilweise erhielten Patienten nach vorausgegangener neoadjuvanter Radiochemotherapie im Rezidiv eine Salvage-Radiochemotherapie. Isolierte Lokalrezidive sind nach neoadjuvanter Therapie und 


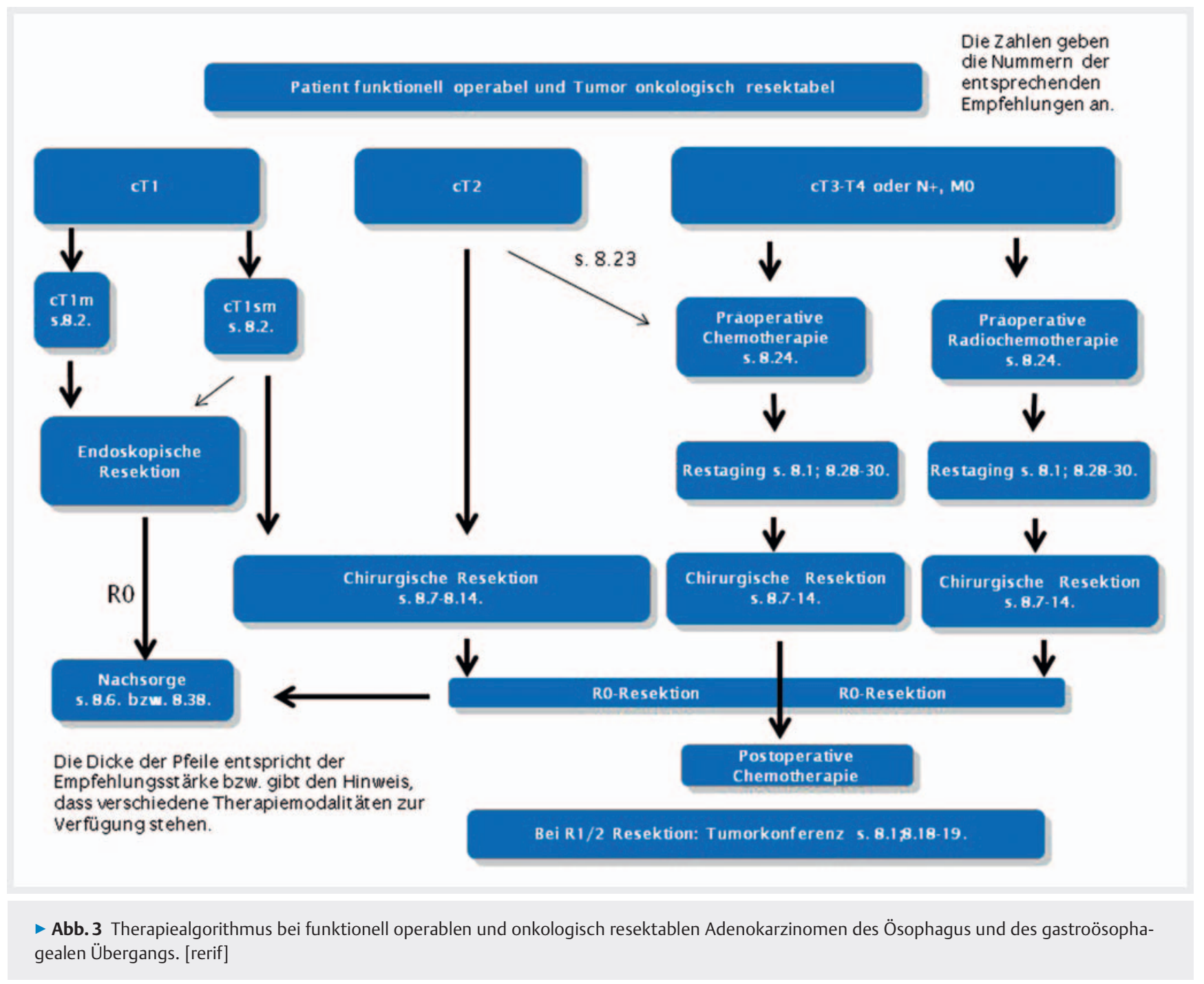

Resektion mit unter $15 \%$ selten [384, 385]. In der Studie von Sudo et al. [384] hatten 5,4\% von 518 Patienten mit lokal fortgeschrittenen Adenokarzinomen des ösophagogastralen Übergangs ein isoliertes Lokalrezidiv nach neoadjuvanter Radiochemotherapie und Operation. Die Rezidivbehandlung war stark von den individuellen Risikofaktoren des einzelnen Patienten und der Rezidivlokalisation abhängig. 44\% der Patienten mit isoliertem lokoregionalen Rezidiv erhielten eine Radiochemotherapie bei Rezidiven außerhalb der initial bestrahlten Volumina. Die 3-Jahre-Überlebensrate dieser Patienten betrug 25 \% nach der Rezidivtherapie. Die Mehrzahl der Patienten entwickelten im Verlauf Fernmetastasen.

\subsection{Multimodale Therapiekonzepte}

\begin{tabular}{|l|l|l|}
\hline 8.21. & Konsensbasierte Empfehlung & $\begin{array}{l}\text { geprüft } \\
\mathbf{2 0 1 8}\end{array}$ \\
\hline EK & $\begin{array}{l}\text { Wenn eine neoadjuvante Therapie vorgesehen ist, soll vor } \\
\text { Beginn der Therapie bei den Patienten eine Risikoanalyse } \\
\text { wichtiger Organfunktionen und ein Screening auf Man- } \\
\text { gelernährung durchgeführt werden. }\end{array}$ \\
\hline Konsens- & Konsens (91\%) \\
\hline stärke & \\
\hline
\end{tabular}

\section{Hintergrund}

Vor jeder potenziell belastenden Therapie ist der zu erwartende Nutzen gegenüber dem möglichen Risiko abzuwägen, daher sollte das Risiko einer Therapie zuvor durch Untersuchung der Organfunktionen abgeschätzt werden. Ebenso ist eine Mangelernährung mit erhöhter OP-Morbidität assoziiert (vgl. Empfehlung 8.14.). 
Die Dicke der Pfeile entspricht der Empfehlungsstärke bzw. gibt den Hinweis, dass verschiedene Ther apiemodalit äten zur

verfügung stehen.

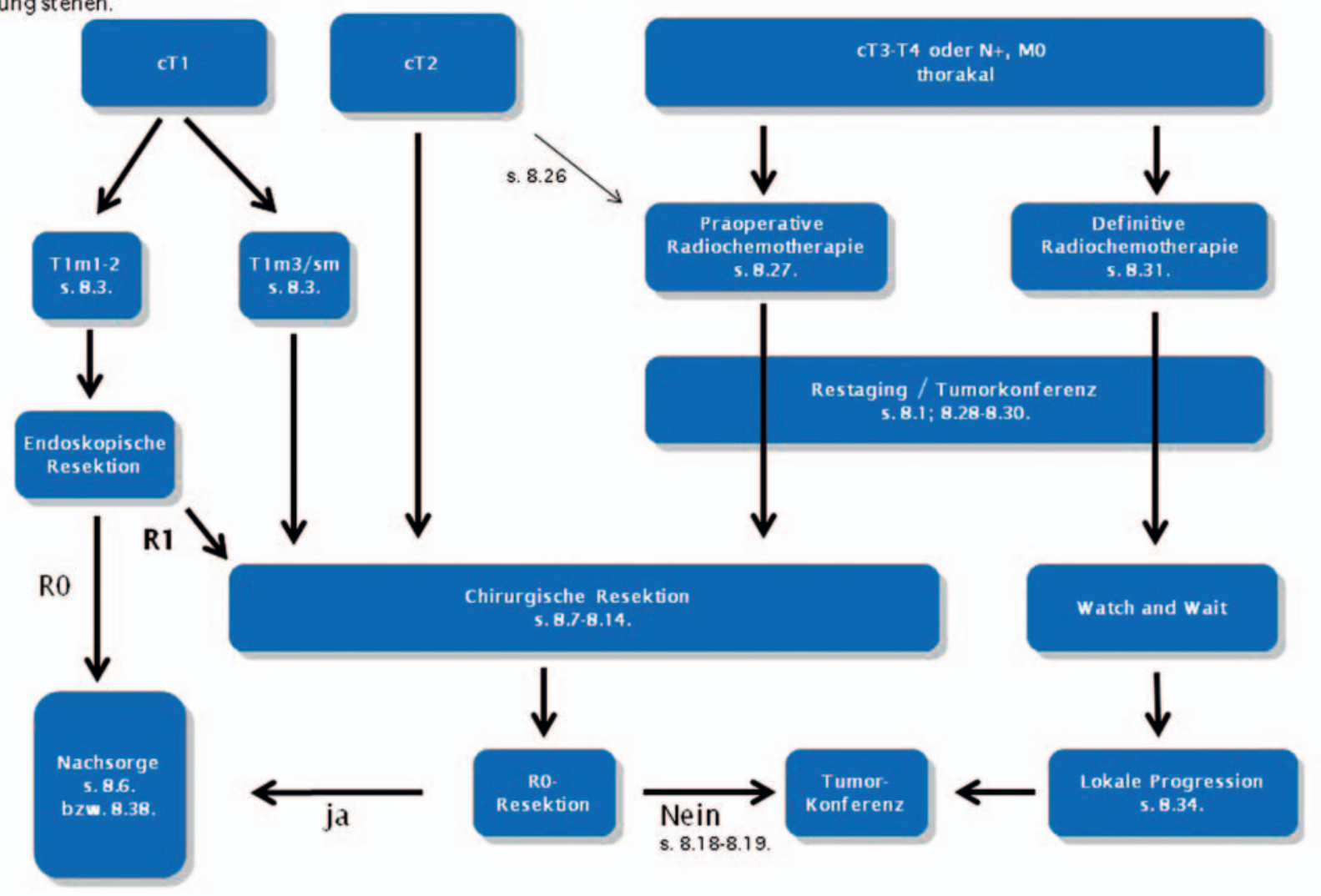

- Abb. 4 Therapiealgorithmus bei funktionell operablen und onkologisch resektablen Plattenepithelkarzinomen des Ösophagus. Zur Therapie mittels definitiver Radiochemotherapie bei lokalisierten Plattenepithelkarzinomen des zervikalen Ösophagus siehe Empfehlung 8.32. [rerif]

\subsubsection{Präoperative Radiotherapie}

\begin{tabular}{|l|l|l|}
\hline $\mathbf{8 . 2 2 .}$ & Evidenzbasierte Empfehlung & $\begin{array}{l}\text { geprüft } \\
\mathbf{2 0 1 8}\end{array}$ \\
\hline $\begin{array}{l}\text { Empfeh- } \\
\text { lungsgrad } \\
\mathbf{0}\end{array}$ & $\begin{array}{l}\text { Eine alleinige präoperative Strahlentherapie kann beim } \\
\text { operablen Patienten mit einem resektablen Ösophagus- } \\
\text { karzinom nicht empfohlen werden. }\end{array}$ \\
\hline $\begin{array}{l}\text { Level of } \\
\text { Evidence } \\
\mathbf{2 a}\end{array}$ & Literatur: $[386-388]$ \\
\hline $\begin{array}{l}\text { Konsens- } \\
\text { stärke }\end{array}$ & Starker Konsens (95\%) \\
\hline
\end{tabular}

\section{Hintergrund}

Malthaner et al. [386] analysierten für das Cancer Care Ontario's Program die randomisierten Studien zur präoperativen Strahlentherapie im Vergleich zur alleinigen Operation beim Ösophaguskarzinom. In dieser Metaanalyse wurden die Überlebensraten nach einem Jahr von 5 randomisierten Studien für beide Therapiearme verglichen. Alle randomisierten Studien wurden vor 1988 beendet. Insgesamt gingen in diese Analyse Daten von 716 Patienten ein. Eine identifizierte Studie wurde ausgeschlossen, da hier keine Überlebensdaten nach einem Jahr verfügbar waren. Das relative Risiko im ersten Jahr zu versterben betrug nach präoperativer Strahlentherapie 1,01 (95\% KI 0,88-1,16) im Vergleich zur alleinigen Operation. Somit wurde kein Vorteil für eine neoadjuvante Strahlentherapie im Vergleich zur alleinigen Operation bei Patienten mit operablem Ösophaguskarzinom gefunden. In einer zweiten Metaanalyse wurden individuelle Überlebensdaten von 1038 Patienten aus 5 randomisierten Studien mit bis zu 10 Jahre Nachbeobachtungszeit ausgewertet [387]. Die beiden Metaanalysen stimmten im Einschluss von 4 Studien überein, unterschieden sich aber bei der fünften Studie. Plattenepithelkarzinome waren die vorherrschende histologische Gruppe in den eingeschlossenen Studien und wurden bei $86 \%$ diagnostiziert. Jedoch zeigte keine der Studien eine nach heutigen Standards adäquate Dosierung der Strahlentherapie. In drei der Studien wurden Strahlendosen von über 3,0 Gy pro Tag verwendet. In zwei Studien wurden sehr geringe Gesamtdosen von 20 Gy mit 2,0 Gy pro Fraktion oder von 35 Gy mit 1,75 Gy pro Fraktion appliziert. 
In dieser Metaanalyse wurde eine Hazard-Ratio von 0,91 (95\% KI 0,80-1,04) zugunsten der präoperativen Strahlentherapie errechnet. Da das Konfidenzintervall die 1 schneidet, konnte auch hier ein Überlebensvorteil durch eine präoperative Radiotherapie nicht nachgewiesen werden.

Neben den randomisierten Studien wurde eine größere vergleichende retrospektive Analyse zur präoperativen Strahlentherapie beim Ösophaguskarzinom mit Daten der Surveillance, Epidemiology, and End Results (SEER) Datenbank des Nationalen Krebsregisters der USA durchgeführt [388]. In dieser Datenbank wurden 441 Patienten mit einem Ösophaguskarzinom der Kategorien cT2-cT4 ohne Fernmetastasen gefunden, die mit präoperativer Strahlentherapie behandelt wurden. Im gleichen Zeitraum wurden 592 Patienten mit alleiniger Operation identifiziert. Adenokarzinome waren der vorherrschende histologische Tumortyp (bei $67 \%$ der Patienten). Patienten mit präoperativer Strahlentherapie zeigten hier in der multivariaten Analyse einen signifikanten Überlebensvorteil mit einem Hazard Ratio von 0,67 (0,57-0,75). Daten zur begleitenden Chemotherapie waren aber nicht in der SEER Datenbank verzeichnet, sodass eine simultane Chemotherapie bei einer Vielzahl der Patienten nicht ausgeschlossen werden konnte. Auch kann ein Selektionsbias innerhalb einer derartigen retrospektiven Studie nicht ausgeschlossen werden. Insgesamt ist die Evidenz für einen Überlebensvorteil mit einer präoperativen Radiochemotherapie beim Plattenepithelkarzinom deutlich besser als mit einer alleinigen präoperativen Strahlentherapie. Beim Adenokarzinom gilt dies für die perioperative Chemotherapie und die präoperative Radiochemotherapie.

In eine unizentrische Phase II-Studie eines Zentrums in China wurden zwischen 2002 und 2003 insgesamt 98 operable Patienten mit lokalisiertem Plattenepithelkarzinom des Ösophagus randomisiert [382]. Offenbar war auch die Aufnahme von Patienten mit einem isolierten Rezidiv im Bereich der Anastomose erlaubt $(n=24)$. Die Patienten erhielten eine dreidimensional geplante konformale Radiotherapie von 46-50 Gy mit anschließendem Boost bis 62-70 Gy. Da alle Patienten letztlich eine kurative Resektion erhielten, spricht vieles dafür, dass Patienten ausgeschlossen wurden, die nicht zur OP kamen oder bei denen keine R0-Resektion möglich war (nicht berichtet). Die Studie untersuchte, ob eine gleichzeitige wöchentliche Chemotherapie mit $30 \mathrm{mg} / \mathrm{m}^{2}$ Cisplatin (Arm B, n=49) gegenüber einer alleinigen präoperativen Radiotherapie (Arm A, $n=49$ ) das Überleben der Patienten verbessert. Der Statistikplan ist nicht dargestellt.

Die kombinierte Chemoradiotherapie verbesserte im Trend die mediane Überlebenszeit (19 vs. 35 Monate, $p=0,051$ ) und das Langzeitüberleben nach 5 Jahren $(p=0,051)$. Die zusätzliche Chemotherapie mit Cisplatin erwies sich in einer multivariaten Analyse als unabhängiger Prognosefaktor. Die Studie weist erhebliche methodische Schwächen auf (unizentrisch, geringe Patientenzahl, Patienten mit Rezidiv zugelassen, unklare Stadienverteilung vor Therapie, unklare Dosisverteilung der präoperativen Bestrahlung zwischen den Behandlungsarmen) [382].

Die Ergebnisse dieser Studie haben nach Meinung der Arbeitsgruppe keinen Einfluss auf die Empfehlung 8.22, welche eine präoperative Radiotherapie beim resektablen SCC des Ösophagus nicht empfiehlt.

\subsubsection{Präoperative Radiochemotherapie und perioperative} Chemotherapie

\begin{tabular}{|c|c|c|}
\hline 8.23. & Evidenzbasierte Empfehlung & $\begin{array}{l}\text { geprüft } \\
2018\end{array}$ \\
\hline $\begin{array}{l}\text { Empfeh- } \\
\text { lungsgrad } \\
\mathbf{0}\end{array}$ & \multicolumn{2}{|c|}{$\begin{array}{l}\text { Bei lokalisierten Adenokarzinomen des Ösophagus und } \\
\text { des ösophagogastralen Übergangs der Kategorie cT2 } \\
\text { kann eine präoperative Chemotherapie durchgeführt und } \\
\text { postoperativ fortgesetzt werden. }\end{array}$} \\
\hline $\begin{array}{l}\text { Level of } \\
\text { Evidence } \\
\text { 1b }\end{array}$ & \multicolumn{2}{|l|}{ Literatur: [18, 389 - 395] } \\
\hline $\begin{array}{l}\text { Konsens- } \\
\text { stärke }\end{array}$ & \multicolumn{2}{|l|}{ Konsens (91\%) } \\
\hline
\end{tabular}

\section{Hintergrund}

Hierbei handelt es sich um eine Leitlinienadaptation [18]. In den vorliegenden randomisierten Studien zur prä- oder perioperativen Chemotherapie bei Ösophaguskarzinomen ist der Anteil an Patienten mit einer initialen T1/2 Kategorie entweder nicht angegeben [391 - 394] oder, soweit aus der primär operierten Patientengruppe extrapolierbar, sehr klein (32\% Ychou 2011, 36,8\% Cunningham 2006, 9 \% Law 1997) [395]. Es gibt keine separaten Daten über den Nutzen einer prä- oder perioperativen Therapie bei dieser kleinen Subgruppe der Patienten. Aufgrund einer geringeren Rate an Lymphknotenmetastasierung und occulten Fernmetastasen ist die T2 Kategorie prognostisch günstiger als T3/4 und ein zu erwartender Effekt einer neoadjuvanten Therapie wahrscheinlich geringer. Dennoch waren auch Patienten mit T2 Tumoren Teil der Studienpopulation, bei der durch eine perioperative Chemotherapie ein Überlebensgewinn erzielt werden konnte [389, 390].

Die Empfehlungsstärke zur perioperativen Chemotherapie ist jedoch aufgrund der geringen Patientenzahl schwächer („kann“Empfehlung). Da die beste Evidenz für den Nutzen einer Chemotherapie aus Studien mit perioperativer Applikation der Chemotherapie herrührt [389, 390], wird die postoperative Fortsetzung der Chemotherapie empfohlen.

Vor Einleitung einer postoperativen Chemotherapie im Rahmen eines perioperativen Therapiekonzeptes sollte mit einfachen Mitteln (Röntgen-Thorax, Sonografie Abdomen) eine Metastasierung ausgeschlossen werden. Im Falle des Nachweises einer Metastasierung ist es nicht sinnvoll, das in kurativer Intention präoperativ begonnene Chemotherapiekonzept fortzusetzen. In den veröffentlichten Phase III-Studien zur perioperativen Chemotherapie bei Adenokarzinomen wurde die Chemotherapie bei Metastasennachweis beendet [389, 390].

Folgende Überlegungen sollten bei der Entscheidungsfindung bzgl einer präoperativen Therapie von T2 Ösophaguskarzinomen berücksichtigt werden.

a) In den positiven Studien zur präoperativen Therapie waren jeweils auch T2 Tumoren eingeschlossen und zeigten in Subgruppenanalysen ebenfalls einen positiven Effekt [389, 396, 397] 
b) Aufgrund eines Stagingfehlers ist davon auszugehen, dass knapp $50 \%$ der präoperativ als T2N0 eingeschätzten Tumoren im Resektat tatsächlich ein höheres Stadium haben. Markar et al. [398] konnte zeigen, dass 34,7\% im T Stadium und 48,1\% im N Stadium präoperativ einem zu niedrigen Stadium zugerechnet wurden. Bei Speicher et al. [399] waren 41,6\% präoperativ in ein zu niedriges Stadium eingeteilt, bei Crabtree et al. [400], waren von 482 Patienten, die präoperativ als T2NO eingeschätz worden waren $27,4 \%$ im Vergleich zum pathologischen Staging korrekt gestaged, 29,9\% wurden in ein niedrigeres Stadium eingeteilt und 46,7\% wurden höher gestaged, sodass sie eigentlich für eine präoperative Therapie sicher qualifiziert hätten.

Aufgrund dieser Ungenauigkeit im präoperativen Staging, die Patienten eine Chemotherapie vorenthält, die eigentlich, wären sie korrekt diagnostiziert, wie das pathologische Staging am Resektat zeigt, für eine präoperative Therapie qualifiziert hätten, gibt es ein klinisches Dilemma. Theoretisch bestehen 4 mögliche Vorgehensweisen [401]:

1. Der fatalistische Ansatz hält sich an das präoperative Staging und akzeptiert, dass auch im Stadium cT2NO das 5-JahresÜberleben nur $40 \%$ ist.

2. Der pragmatische Ansatz empfiehlt für die Patienten, die am Resektat ein höheres Stadium als initial vermutet diagnostiziert bekommen eine postoperative Therapie. Diese ist allerdings für Plattenepithelkarziome nicht belegt und bei Adenokarzinomen in caucasischen Patienten nur bei weniger als der Hälfte durchführbar [390, 396]

3. Der agressive Ansatz behandelt auch $T 2$ NO Patienten mit einer präoperativen Therapie und baut darauf, dass bei den Patienten, die dadurch eventuell eine Überbehandlung bekommen, eine präoperative Therapie in Studien zumindest nicht schadet.

4. Der wissenschaftliche Ansatz versucht mittels elaborierter Staginguntersuchungen (PET-CT, Feinnadelpunktion) und Biomarkerm (MSI) die aktuell noch nicht validiert sind, Hinweise zu finden, welche Patienten von einer präoperativen Therapie profitieren.

\begin{tabular}{|l|l|l|}
\hline $\mathbf{8 . 2 4 .}$ & Evidenzbasierte Empfehlung & $\begin{array}{l}\text { geprüft } \\
\mathbf{2 0 1 8}\end{array}$ \\
\hline $\begin{array}{l}\text { Empfeh- } \\
\text { lungsgrad } \\
\text { A }\end{array}$ & $\begin{array}{l}\text { Beim operablen Patienten mit Adenokarzinom des } \\
\text { Ösophagus oder des ösophagogastralen Übergangs der } \\
\text { Kategorie cT3 und bei resektablen cT4 Tumoren soll eine } \\
\text { perioperative Chemotherapie oder eine präoperative } \\
\text { Radiochemotherapie durchgeführt werden. }\end{array}$ \\
\hline $\begin{array}{l}\text { Level of } \\
\text { Evidence } \\
\mathbf{1 a}\end{array}$ & {$[388,389,391-393,402-404]$} \\
\hline $\begin{array}{l}\text { Konsens- } \\
\text { stärke }\end{array}$ & Starker Konsens $(100 \%)$ \\
\hline
\end{tabular}

\begin{tabular}{|c|c|c|}
\hline 8.25 . & Evidenzbasierte Empfehlung & $\begin{array}{l}\text { geprüft } \\
2018\end{array}$ \\
\hline $\begin{array}{l}\text { Empfeh- } \\
\text { lungsgrad } \\
\text { B }\end{array}$ & \multicolumn{2}{|c|}{$\begin{array}{l}\text { Die Durchführung einer alleinigen neoadjuvanten Che- } \\
\text { motherapie ohne simultane Radiotherapie beim Platten- } \\
\text { epithelkarzinom des Ösophagus kann nicht empfohlen } \\
\text { werden. }\end{array}$} \\
\hline $\begin{array}{l}\text { Level of } \\
\text { Evidence } \\
\text { 1a }\end{array}$ & \multicolumn{2}{|l|}{$[397,405]$} \\
\hline $\begin{array}{l}\text { Konsens- } \\
\text { stärke }\end{array}$ & \multicolumn{2}{|l|}{ Starker Konsens (95\%) } \\
\hline
\end{tabular}

\begin{tabular}{|l|l|l|}
\hline $\mathbf{8 . 2 6 .}$ & Konsensbasierte Empfehlung & $\begin{array}{l}\text { geprüft } \\
\mathbf{2 0 1 8}\end{array}$ \\
\hline EK & $\begin{array}{l}\text { Bei operablen Patienten mit einem Plattenepithelkarzi- } \\
\text { nom des Ösophagus der Kategorie cT2 kann eine prä- } \\
\text { operative Radiochemotherapie mit anschließender } \\
\text { kompletter Resektion durchgeführt werden. }\end{array}$ \\
\hline Konsens- & Starker Konsens (100\%) \\
\hline stärke & \\
\hline
\end{tabular}

\begin{tabular}{|l|l|l|}
\hline $\mathbf{8 . 2 7 .}$ & Evidenzbasierte Empfehlung & $\begin{array}{l}\text { geprüft } \\
\mathbf{2 0 1 8}\end{array}$ \\
\hline $\begin{array}{l}\text { Empfeh- } \\
\text { lungsgrad } \\
\text { A }\end{array}$ & $\begin{array}{l}\text { Bei operablen Patienten mit einem Plattenepithelkarzi- } \\
\text { nom des Ösophagus der Kategorie cT3 und bei resektab- } \\
\text { len cT4 Tumoren soll eine präoperative Radiochemothe- } \\
\text { rapie mit anschließender kompletter Resektion } \\
\text { durchgeführt werden. }\end{array}$ \\
\hline $\begin{array}{l}\text { Level of } \\
\text { Evidence } \\
\mathbf{1 a}\end{array}$ & $\begin{array}{l}\text { Literatur: [406-409] } \\
\text { Konsens- } \\
\text { stärke }\end{array}$ & \begin{tabular}{l} 
Starker Konsens (96\%) \\
\hline
\end{tabular}
\end{tabular}

\section{Hintergrund}

Zur prognostischen Bedeutung der präoperativen Therapie wurde vom Ärztlichen Zentrum für Qualität in der Medizin (ÄZQ) ein Evidenzbericht erstellt, der Publikationen bis Februar 2013 einschloss. Hinsichtlich einer präoperativen Radiochemotherapie konnten 17 systematische Reviews/Metaanalysen und 13 randomisierten klinische Studien (RCT) beurteilt werden. 7 RCTs wurde eine hohe Qualität bescheinigt (3 RCTs Evidenzlevel 1b mit engem Konfidenzintervall: [406 - 408] und 4 RCTs Evidenzlevel 1b(-) mit weitem Konfidenzintervall [409-412]. Zur präoperativen Chemotherapie lagen Vollpublikationen aus 15 systematischen Reviews/Metaanalysen und 6 RCTs vor (davon 3 von hoher Qualität mit engem Konfidenzintervall, Evidenzlevel 1b: [389, 391, 392]. 


\section{Präoperative Radiochemotherapie}

Insgesamt ergeben sich statistisch signifikante Vorteile mit kombinierter präoperativer Radiochemotherapie aus Cisplatin/ 5-FU teilweise plus dritter Substanz oder Carboplatin/Paclitaxel und Radiotherapie mit einer Dosis bei 40 - 50,4 Gy in konventioneller Fraktionierung mit 1,8 Gy bis 2,0 Gy pro Fraktion gegenüber alleiniger Operation. Analog zur Chemotherapie bei ösophagogastralen Adenokarzinomen kann offenbar auch in der kombinierten Radiochemotherapie Cisplatin durch Oxaliplatin ersetzt werden. Jedenfalls konnte eine randomisierte Phase II/III Studie unter Einschluss von Adenokarzinomen (14\%) und Plattenepithelkarzinomen (86\%) die Gleichwertigkeit von FOLFOX gegenüber Cisplatin/5-FU im Rahmen einer definitiven Radiochemotherapie (ohne OP) belegen. Die Toxizität war ebenfalls nicht unterschiedlich [413].

Im Einzelnen konnten statistisch signifikante Ergebnisse zugunsten der neoadjuvanten Radiochemotherapie in 6/13 RCTs erreicht werden [406, 409, 411, 412, 414, 416-418].

Bezogen auf den priorisierten Endpunkt „Überleben“ (Überlebensrate nach 3 oder 5 Jahren bzw. medianes Überleben) wiesen 5 Studien einen signifikanten Vorteil für die präoperative Radiochemotherapie gegenüber alleiniger Operation nach [409, 414, 416, 419].

Die „negativen“ Studien verglichen die präoperative Radiochemotherapie entweder nicht mit alleiniger Operation [410] und/ oder die Zahl der eingeschlossenen Patienten war (zu) gering, um einen Vorteil statistisch nachzuweisen [407, 408, 410, 412, $420,421]$. Wo dies angegeben wurde, führte die präoperative Radiochemotherapie zu einer Reduktion der Lokalrezidivrate; in 2 Studien [408, 412] war dies statistisch signifikant. Schwere oder lebensbedrohliche Nebenwirkungen traten durch die Radiochemotherapie bei $13 \%$ bis $57 \%$ der Patienten auf. Todesfälle waren jedoch sehr selten (in der Regel unter $1 \%$, bei Tepper 2008 [409] (4\%). Die perioperative Letalität war in einer randomisierten Studie nach Radiochemotherapie + OP signifikant höher als nach primärer Resektion [408]. Dies betrifft aber ausschließlich Patienten mit Plattenepithelkarzinomen (entsprechend der Lokalisation im oberen und mittleren Thoraxdrittel) und ist insbesondere auf heute nicht mehr verwendete hypofraktionierte Schemata der Strahlentherapie zurückzuführen. Nur wenn man Studien mit solch veralteten Fraktionierungs-Schemata berücksichtigt, ergibt sich nach einer jüngsten Metaanalyse [422] eine signifikant erhöhte postoperative Letalität nach präoperativer Radiochemotherapie für Plattenepithelkarzinome (HR 1,95, 95 \% KI 1,06-3,60), nicht aber für Studien mit Adenokarzinomen (HR 0,79, $95 \% \mathrm{KI}$ 0,39 - 1,61). Insbesondere wird auch die Rate an Anastomoseninsuffizienzen durch die präoperative Radiochemotherapie nicht erhöht (HR 1,00).

Radiochemotherapieeffekt bei unterschiedlichen histologischen Subtypen

In Metaanalysen scheint der Effekt einer präoperativen Radiochemotherapie bei Plattenepithelkarzinomen des Ösophagus im Vergleich zu Adenokarzinomen des Ösophagus ähnlich zu sein (Plattenepithelkarzinom HR 0,80, 95 \% KI 0,68 - 0,93; Adenokarzinom HR 0,75, 95 \% KI 0,59-0,95, [397]. In dieser Metaanalyse ist allerdings die Studie von van Hagen 2012 [414] für den Subgruppenvergleich von Adenokarzinom und Plattenepithelkarzinom nicht berücksichtigt, bei der der Effekt für Plattenepithelkarzinome deutlich höher war.

Klinische Bewertung zum Einsatz einer präoperativen Radiochemotherapie bei Adeno- und Plattenepithelkarzinomen

Eine präoperative Radiochemotherapie verbessert konsistent das Überleben sowohl bei Plattenepithelkarzinomen als auch bei Adenokarzinomen und soll daher durchgeführt werden. Bei Adenokarzinomen stellt die alleinige Chemotherapie eine ebenso wirksame Alternative dar (siehe unten).

\section{Fazit präoperative Radiochemotherapie}

Die Mehrzahl der systematischen Übersichtsarbeiten weist einen signifikanten Überlebensvorteil unter präoperativer Radiochemotherapie plus Operation gegenüber alleiniger Operation nach. Gleichzeitig werden erhöhte R0-Resektionsraten und geringere lokoregionale Rezidivneigung berichtet. Die postoperative Letalität kann bei Verwendung hypofraktionierter Strahlentherapieschemata erhöht sein. Die Ergebnisse der Publikationen mit aggregierter Evidenz basieren auf sehr heterogenen Einzelstudien, was aus methodischer Sicht kritisch zu bewerten ist. Deshalb lassen sich die Aussagen nicht durchgängig auf einen histologischen Typ, bestimmte TNM-Stadien, bestimmte Höhenlokalisationen des Tumors und bestimmte Regime der Chemotherapie oder der Radiotherapie eingrenzen. Eine Cochrane-Analyse bezüglich des Effektes einer Chemo- oder Radiochemotherapie bei Adenokarzinomen [423], die erst nach Erstellung des Evidenzberichts publiziert wurde, weist die Überlebensvorteile für die präoperative Therapie von Adenokarzinomen vor allem bei einer Lokalisation im ösophagogastralen Übergang nach (HR 0,69 gegenüber Adenokarzinomen des Ösophagus HR 0,78 und des Magens HR 0,94). Ferner scheint der prognostische Vorteil durch eine präoperative Radiochemotherapie höher (HR 0,70, 95 \% 0,50-0,99, basierend auf 389 Patienten) als nach präoperativer Chemotherapie (HR 0,83, $95 \%$ KI 0,75-0,91, basierend auf 2033 Patienten). Die Qualität dieser Evidenz wurde von den Autoren aufgrund der unterschiedlichen Patientenzahlen für die Radiochemotherapie bei Adenokarzinomen allerdings als moderat, für die Chemotherapie als hoch eingeschätzt.

Die Mehrheit der eingeschlossenen Einzelstudien erhielt eine aufgrund methodischer Limitationen herabgestufte Bewertung. Aus 13 RCTs berichten fünf statistisch signifikante Überlebensraten zugunsten der Radiochemotherapie. Nur eine dieser Studien ist von guter methodischer Qualität [411] und ihr Ergebnis daher als valide einzuschätzen. In dieser Studie war der Überlebensvorteil durch die präoperative Radiochemotherapie bei Plattenepithelkarzinomen höher (HR 0,45: 0,23-0,84) als bei Adenokarzinomen (HR 0,73: 0,52-0,998).

\section{Präoperative Chemotherapie}

Insgesamt weisen 5 Studien mit einer präoperativen Chemotherapie aus Cisplatin und 5-FU einen signifikanten Vorteil gegenüber alleiniger Operation aus [18, 389, 392 - 395, 424]. Bezüglich des priorisierten Endpunkts „Überleben“ belegen die britische Studie (Allum 2009 [393]: 0,84; $95 \%$ KI 0,72-0,98, p=0,03), die holländische Studie (Boonstra 2011 [392]: HR=0,71, $95 \% \mathrm{KI}$ $0,51-0,98, p=0,03$ ) und die französische Studie [389]: HR 0,69, $95 \% \mathrm{KI} 0,50-0,95, \mathrm{p}=0,02$ ) einen signifikanten Vorteil durch die präoperative Chemotherapie (+ postoperativ bei Ychou 2011 [389]). Der Anteil an Patienten mit einem Adenokarzinom lag in 
diesen positiven Studien bei 66,5\% [393], $100 \%$ [389] und $0 \%$ [392]. Die Rate an R0-Resektionen war in 4 Studien signifikant erhöht [389, 394, 395, 424]. Schwere oder lebensbedrohliche Nebenwirkungen traten durch die präoperative Chemotherapie bei $29 \%$ bis $40 \%$ der Patienten auf. Therapiebedingte Todesfälle waren sehr selten, lediglich in der MRC Studie waren präoperative Todesfälle im Chemotherapiearm signifikant erhöht (MRC 2002). Die postoperative Letalität wurde durch die präoperative Therapie nicht erhöht. Ebenso gibt es keinen Unterschied in der therapiebedingten Letalität (prä- und postoperativ) wenn eine Chemotherapie der Operation vorausgegangen ist [422] (HR 1,20, $95 \% \mathrm{KI}$ $0,71-2,03)$. In den Evidenzbericht ist die Magic-Studie [390] nicht eingeflossen, da vornehmlich Magenkarzinome eingeschlossen waren. In dieser Studie wiesen jedoch von den 501 eingeschlossenen Patienten 131 Patienten Tumoren des Ösophagus und ösophagogastralen Übergangs auf, bei denen sich ein ähnlich positiver Effekt zeigte wie in der Gesamtpopulation der Studie.

In eine unizentrische Phase III-Studie eines universitären Zentrums in China wurden zwischen 2005 und 2007 insgesamt 346 operable Patienten mit resektablem Plattenepithelkarzinom des Ösophagus randomisiert [425]. Die Studie untersuchte, ob eine zusätzliche postoperative Chemotherapie (Arm A, $n=173$ ) gegenüber einer alleinigen präoperativen Chemotherapie (Arm $B, n=170$ ) das Langzeitüberleben verbessert. Ziel war es, die Überlebensrate nach 5 Jahren von $20 \%$ auf $35 \%$ zu erhöhen. Die Publikation gibt keine Information darüber, wie die Verteilung der Tumorstadien zwischen den Therapiearmen ausfiel (EUS nicht durchgeführt). Lediglich eine Gleichverteilung hinsichtlich des Tumordurchmessers wird dokumentiert. Die Chemotherapie bestand aus einer Dreifachkombination aus Cisplatin, 5-FU und Paclitaxel. Trotz dieser intensiven Kombination konnten im Arm A $69 \%$ der Patienten die komplette postoperative Chemotherapie (2 Kurse) erhalten. In beiden Armen kamen über $90 \%$ der Patienten zur Operation (92\% vs. $93 \%$ ). Bei $83 \%$ der Patienten gelang eine kurative Resektion nach präoperativer Chemotherapie, mit einer Rate an komplettem histologischem Ansprechen von $24 \%$ aller operierten Patienten. Das Gesamtüberleben war nach perioperativer Chemotherapie (Arm A) signifikant höher (nach 5 Jahren $38 \%$ vs. $22 \%$; HR 0,79 (0,59-0,95), p<0,001). Die Überlegenheit wurde auch für das Rezidiv-freie Überleben nachgewiesen (HR 0,62) [425].

Die Ergebnisse dieser Studie haben nach Meinung der Arbeitsgruppe keinen Einfluss auf die Empfehlung 8.25, die sich gegen eine präoperative Chemotherapie beim SCC des Ösophagus ausspricht. In erster Linie bleibt die Empfehlung unverändert bestehen, weil die genannte Publikation die Fragestellung einer präoperativen Chemotherapie im Vergleich zur alleinigen Operation nicht geprüft hat. Allerdings deuten die Daten darauf hin, dass eine alleinige präoperative Chemotherapie beim lokalisierten SCC nicht optimal ist. Die Studie weist zudem einige relevante Schwächen auf, die zur Vorsicht bei der Interpretation raten lassen. Insbesondere das offensichtlich ungenaue, jedenfalls in der Publikation nicht dargestellte Tumorstadium der Patienten (CT als Basis, keine EUS) eröffnet ein hohes Risiko für einen Bias, der das Gesamtergebnis in Frage stellt. Ob eine perioperative Chemotherapie das Langzeitüberleben von Patienten mit resektablem SCC des Ösophagus verbessern kann, sollte im Rahmen einer pro- spektiven Studie gegenüber präoperativer Chemoradiotherapie untersucht werden.

Die FLOT4-Studie der AIO untersuchte an 716 Patienten mit resektablem Magenkarzinom oder Adenokarzinom des gastroösophagealen Übergangs, ob die Hinzunahme von Docetaxel (FLOT, 5-FU/Oxaliplatin/Docetaxel) in die perioperative Therapie zu einer Verbesserung gegenüber ECF führt [396, 426]. Die 2016 im Lancet Oncology publizierte Zwischenanalyse der Phase II zeigte bereits deutlich bessere histopathologisch komplette Remissionsraten im FLOT-Arm mit 16\% im Vergleich zum ECF (ECX)-Arm mit $6 \%(p=0,015)$, wobei die Verbesserung insbesondere bei Patienten mit einem intestinalen Tumortyp nach Lauren zu verzeichnen war (23\% vs. $10 \%$ ) [426]. Die Überlebensdaten des Phase-III-Teils wurden 2017 vorgestellt. Das mediane Gesamtüberleben fiel mit 50 gegenüber 35 Monaten und einer Hazard Ratio von 0,77 $(p=0,012)$ ebenso deutlich zugunsten des neuen Regimes aus wie das progressionsfreie Überleben (30 vs. 18 Monate, HR 0,75; $p=0,004)$ [396]. Der Nutzen des FLOT-Regimes war in allen untersuchten Subgruppen gleichermaßen zu sehen, ebenso bei Patienten mit Siegelringzellkarzinomen. Die Rate an operativer Morbidität und Mortalität waren ebenfalls nicht erhöht [396]. Das FLOTProtokoll definierte damit einen neuen Standard in der kurativen Therapie des Adenokarzinoms des Magens und des gastroösophagealen Übergangs. Diese Studie hat auch für Ösophaguskarzinome Relevanz, da 393 der Patienten Adenokarzinome des gastroösophagealen Übergangs hatten, davon 160 AEG I. Im Forrest Plot war die Hazard Radio für AEG 1 Tumoren 0,60 mit einem signifikanten Konfidenzintervall. Daher stellt FLOT auch für Adenokarzinome des distalen Ösophagus bei Entscheid für eine perioperative Chemotherapie den Therapiestandard dar.

Chemotherapieeffekt bei unterschiedlichen histologischen Subtypen

Die Evidenz für den Effekt einer präoperativen/perioperativen Chemotherapie ist bei Plattenepithelkarzinomen unterschiedlich zu Adenokarzinomen. Während in Metaanalysen bei Adenokarzinomen ein signifikanter Überlebensgewinn durch präoperative Chemotherapie erreicht werden kann (HR 0,83, 95 \% KI 0,71 0,95 , [427], ist dieser für Plattenepithelkarzinome nicht signifikant nachzuweisen (HR 0,92; $95 \%$ KI 0,81 - 1,04, [397]; HR 0,93, $95 \% \mathrm{KI}$ 0,81-1,08, [428]. Bei Adenokarzinomen ist der Effekt einer präoperativen Chemotherapie unstrittig. Bei Plattenepithelkarzinomen lässt sich ein konsistenter Überlebenszugewinn trotz der positiven Studie von Boonstra [392] in Metaanalysen nicht belegen. In den Metaanalysen nicht enthalten war eine japanische Multicenterstudie mit 330 Patienten, welche die Chemotherapie prä- versus postoperativ bei resektablen Plattenepithelkarzinomen vergleicht [429] und einen signifikanten Überlebensvorteil für die präoperative Therapie ( $\mathrm{HR} 0,73, \mathrm{p}=0,04)$ bei geringerer Toxizität aufweist.

\section{Klinische Bewertung}

Bei Adenokarzinomen wird konsistent durch eine präoperative Chemotherapie ein Überlebensvorteil nachgewiesen. Die überzeugendste Arbeit hoher Qualität [389] sowie die britische Magic-Studie [390] und die deutsche FLOT 4 Studie [396] haben die Chemotherapie prä- und postoperativ verabreicht. Aus diesem Grund soll bei Adenokarzinomen des Ösophagus und gastroöso- 
phagealen Übergangs eine Chemotherapie präoperativ begonnen und postoperativ fortgesetzt werden.

Bei Plattenepithelkarzinomen sind trotz günstiger Effekte auf die R0-Resektionsrate keine konsistenten signifikanten Überlebensverbesserungen durch eine Chemotherapie zu erzielen. Daher gibt es keine überzeugende Evidenz die ihren Einsatz insbesondere im Vergleich zur präoperativen Radiochemotherapie empfehlenswert erscheinen lässt.

\section{Fazit präoperative Chemotherapie}

Die Ergebnisse der systematischen Übersichtsarbeiten basieren auf sehr heterogenen Einzelstudien. Aus methodischer Sicht sind diese daher kritisch zu bewerten [18, 404]. Wie bei der neoadjuvanten Radiochemotherapie lassen sich diese Aussagen nicht durchgängig auf einen histologischen Typ, bestimmte TNM-Stadien, bestimmte Höhenlokalisationen des Tumors oder bestimmte Chemotherapieregime eingrenzen. Dort wo entsprechende Subgruppenanalysen erfolgt sind, weist die präoperative Chemotherapie einen signifikanten Überlebensvorteil für Patienten mit Adenokarzinom, nicht aber mit Plattenepithelkarzinom auf [397, 405]. Die Mehrheit der eingeschlossenen Einzelstudien erhielt eine herabgestufte methodische Bewertung. Aus 6 randomisierten Studien berichten drei statistisch signifikante Überlebensraten zugunsten der präoperativen Therapie. Nur zwei dieser Studien sind von guter methodischer Qualität [389, 392] und ihre Ergebnisse daher als valide einzuschätzen. Es wurden vor allem neoadjuvante Chemotherapieregime mit Cisplatin und 5-FU verwendet. Andererseits berichtet eine Studie mit guter methodischer Qualität keine Effekte im Vergleich von präoperativer Chemotherapie plus OP versus alleinige OP [391]. Etwa die Hälfte der Patienten in dieser Studie hatte ein Plattenepithelkarzinom. Weiterhin werden statistisch signifikant erhöhte Raten an R0-Resektionen in vier randomisierten Studien berichtet. Eine davon wird als methodisch gut bewertet. Es werden keine statistisch signifikant unterschiedlichen Raten an Fernrezidiven berichtet.

\subsubsection{Restaging nach präoperativer multimodaler Therapie}

\begin{tabular}{|l|l|l|}
\hline $\mathbf{8 . 2 8 .}$ & Konsensbasierte Empfehlung & $\begin{array}{l}\text { geprüft } \\
\mathbf{2 0 1 8}\end{array}$ \\
\hline EK & $\begin{array}{l}\text { Nach Abschluss einer präoperativen Therapie soll ein } \\
\text { erneuter Ausschluss von Fernmetastasen erfolgen. Ein } \\
\text { Restaging des Lokalbefundes kann zur Planung der OP } \\
\text { erfolgen. }\end{array}$ \\
\hline Konsens- & Starker Konsens (100\%) \\
\hline stärke & \\
\hline
\end{tabular}

\begin{tabular}{|l|l|l|}
\hline $\mathbf{8 . 2 9 .}$ & Konsensbasierte Empfehlung & $\begin{array}{l}\text { geprüft } \\
2018\end{array}$ \\
\hline EK & $\begin{array}{l}\text { Falls es unter einer präoperativen Therapie zu klinischen } \\
\text { Zeichen der Tumorprogression kommt, soll eine symp- } \\
\text { tombezogene Diagnostik erfolgen. Wenn endoskopisch } \\
\text { oder bildgebend eine lokale Tumorprogression nachge- } \\
\text { wiesen ist, sollte frühzeitig eine OP durchgeführt werden. }\end{array}$ \\
\hline
\end{tabular}

\begin{tabular}{|l|l|l|}
\hline $\mathbf{8 . 2 9 .}$ & Konsensbasierte Empfehlung & $\begin{array}{l}\text { geprüft } \\
\mathbf{2 0 1 8}\end{array}$ \\
\hline $\begin{array}{l}\text { Level of } \\
\text { Evidence }\end{array}$ & Adaptation Deutsche Leitlinie Magenkarzinom 2012 [18] \\
\hline $\begin{array}{l}\text { Konsens- } \\
\text { stärke }\end{array}$ & Starker Konsens (100\%) \\
\hline
\end{tabular}

\section{Hintergrund}

In den Phase III-Studien zur perioperativen Therapie erfolgte bei fehlendem Hinweis auf Tumorprogress die neoadjuvante Therapie planmäßig und führte in dieser Form für die gesamte Patientengruppe zu einer Verbesserung des Überlebens [389, 390, 410].

Falls im Verlauf der neoadjuvanten Therapie jedoch klinische Hinweise auf einen Tumorprogress bestehen (Verschlechterung tumorbedingter Symptome oder des Allgemeinzustandes) erscheint es sinnvoll, eine symptomorientierte Diagnostik mit erneutem Computertomogramm und Endoskopie durchzuführen. Im Falle eines lokalen Tumorprogresses unter neoadjuvanter Therapie sollte eine frühzeitige Operation durchgeführt werden, da Patienten mit einer lokalen Tumorprogression unter Therapie wahrscheinlich nicht von einem Fortsetzen dieser Therapie profitieren. Bisher gibt es keine validen Daten, die eine Therapieumstellung oder Therapieintensivierung rechtfertigen würden. Die präoperative Therapie sollte jedoch nicht abgebrochen werden, wenn keine Tumorprogression vorliegt (Leitlinienadaptation S3-Leitlinie Magenkarzinom) [18, 404].

\subsubsection{Responseprädiktion}

\begin{tabular}{|l|l|l|}
\hline $\mathbf{8 . 3 0 .}$ & Konsensbasierte Empfehlung & $\begin{array}{l}\text { geprüft } \\
\mathbf{2 0 1 8}\end{array}$ \\
\hline EK & $\begin{array}{l}\text { Der klinische Nutzen der FDG-PET zur Response-Beurtei- } \\
\text { lung einer Chemotherapie oder Radiochemotherapie vor } \\
\text { Operation wird kontrovers beurteilt, weshalb die FDG- } \\
\text { PET/CT bei dieser Fragestellung nicht routinemäßig } \\
\text { durchgeführt werden soll. }\end{array}$ \\
\hline $\begin{array}{l}\text { Konsens- } \\
\text { stärke }\end{array}$ & \begin{tabular}{l} 
Starker Konsens $(96 \%)$ \\
\hline
\end{tabular} \\
\hline
\end{tabular}

\section{Hintergrund}

Der Stellenwert der Fluordeoxyglucose-Positronen-EmissionsTomografie (FDG-PET) zur Vorhersage des Ansprechens auf eine neoadjuvante Chemotherapie wurde von mehreren Arbeitsgruppen untersucht.

Etliche Studien untersuchten die FDG-PET nach neoadjuvanter Radiochemotherapie und vor Tumorresektion mit der Frage der histopathologischen Responseprädiktion und Prognoseabschätzung [430]. In Zusammenschau zeigen die meisten Studien eine mehr oder weniger starke Korrelation zwischen metabolischer Response (FDG-PET) und klinischem oder histopathologischem Ansprechen. Die Prognose metabolischer Responder war in fast allen Studien statistisch signifikant besser als die Prognose metabolischer Non-Responder. Aller- 
dings wurden die entsprechenden Cut-off-Werte bisher nicht in prospektiven Studien validiert und multizentrische Studien fehlen. Schlussendlich erscheint der positive prädiktive Wert des Tests (d. h. die Fähigkeit mittels FDG-PET eine histopathologische Komplettremission vorherzusagen) nicht hoch genug, um daraus Entscheidungen für oder gegen eine chirurgische Resektion abzuleiten.

Andere Autoren untersuchten sequenziell mittels FDG-PET die Veränderung der Tumor-Glukoseaufnahme während neoadjuvanter Chemotherapie oder Radiochemotherapie. Es wurde beobachtet, dass eine Abnahme des sog. Standard Uptake Value (SUV) um $\geq 35 \%$ in Relation zu Baseline innerhalb 2 Wochen nach Start einer neoadjuvanten Chemotherapie eine hohe Genauigkeit zur Vorhersage des histopathologischen Ansprechens nach neoadjuvanter Chemotherapie hat [431-433]. Insbesondere die richtige Erkennung von Nicht-Ansprechern (negativer prädiktiver Wert) war in bis zu $95 \%$ der untersuchten Patienten bemerkenswert hoch [431]. Die metabolische Response während neoadjuvanter Chemotherapie fand sich darüber hinaus mit der Gesamtprognose bei Patienten mit Adenokarzinomen des Ösophagus und des ösophagogastralen Übergangs assoziiert. Frühe metabolische Response (PET-Response) erwies sich als genauer zur Vorhersage von histologischer Regression als das morphologische Ansprechen mittels hochauflösender Mehrschicht-CT [434]. Basierend auf diesen Beobachtungen führte die Münchner Arbeitsgruppe interventionelle Studien durch und konnte erstmals darstellen, dass die frühe PET-Response Grundlage eines individualisierten Behandlungsplans beim Adenokarzinom des Ösophagus und des ösophagogastralen Übergangs sein kann [435, 436]. Bislang konnte aber noch nicht überzeugend dargestellt werden, dass sich durch frühe PET-basierte Therapiealgorithmen die Gesamtprognose der Patienten mit Ösophaguskarzinom verbessern lässt. Auch das Postulat aus den vorausgehenden Studien, dass sich durch PET-Response-basierte Behandlungsalgorithmen unwirksame Chemotherapie einsparen oder zum Wohle des Patienten modifizieren lässt, bedarf einer prospektiv-multizentrischen Validierung.

Darüber hinaus zeigt sich in jüngeren Studien, dass die Aussagefähigkeit der frühen PET-basierten Response während neoadjuvanter Chemotherapie nicht ohne Abstriche auf eine simultane Radiochemotherapie übertragen werden können. Die positive und negative Vorhersagekraft hinsichtlich histopathologischer Regression erwies sich in mehreren Studien als deutlich schwächer [437440]. Dies wird unter anderem auf radiogen induzierte inflammatorische Reaktionen zurückgeführt, welche zu schwierig interpretierbaren Signalalterationen in der FDG-PET führen können.

\subsubsection{Indikation zur definitiven Radiochemotherapie}

\begin{tabular}{|l|l|l|}
\hline 8.31. & Evidenzbasierte Empfehlung & $\begin{array}{l}\text { geprüft } \\
\mathbf{2 0 1 8}\end{array}$ \\
\hline $\begin{array}{l}\text { Empfeh- } \\
\text { lungsgrad } \\
\text { A }\end{array}$ & $\begin{array}{l}\text { Eine definitive Radiochemotherapie soll unabhängig von } \\
\text { der histologischen Entität des Ösophaguskarzinoms er- } \\
\text { folgen, wenn der Tumor im Rahmen einer interdisziplinä- } \\
\text { ren Tumorkonferenz als chirurgisch/endoskopisch nicht } \\
\text { resektabel erachtet wird oder wenn ein Patient funktio- } \\
\text { nell nicht operabel ist bzw. die Operation nach ausführli- } \\
\text { cher Aufklärung ablehnt. }\end{array}$ \\
\hline
\end{tabular}

\begin{tabular}{|c|c|c|}
\hline 8.31. & Evidenzbasierte Empfehlung & $\begin{array}{l}\text { geprüft } \\
2018\end{array}$ \\
\hline $\begin{array}{l}\text { Level of } \\
\text { Evidence } \\
\text { 1b }\end{array}$ & Literatur: [415, 441 - 449] & \\
\hline $\begin{array}{l}\text { Konsens- } \\
\text { stärke }\end{array}$ & Starker Konsens (100\%) & \\
\hline
\end{tabular}

\section{Hintergrund}

Für Patienten, die medizinisch nicht operabel sind oder deren Ösophaguskarzinom als nicht resektabel eingeschätzt wird, besteht, sofern keine Fernmetastasen vorliegen, eine kurative Chance mit definitiver Radiochemotherapie. Sowohl beim Plattenepithelkarzinom als auch beim Adenokarzinom im Stadium II-III wurden in prospektiven Studien [441, 442] als auch in großen Registerstudien [441, 442] Langzeitüberlebensraten um 10 - 35 \% nach 5 Jahren beobachtet. Dabei hängt das Langzeitüberleben von der T-Kategorie nach endoskopischem Ultraschall oder Computertomogramm, der N-Kategorie nach endoskopischen Ultraschall, sowie dem Allgemeinzustand der Patienten ab [442 - 445].

Die definitive Radiochemotherapie ist effektiver als die Strahlentherapie alleine, weshalb bei Patienten ohne Kontraindikationen gegen eine Cisplatin-, Carboplatin-, oder Oxaliplatin-haltige Chemotherapie immer die Kombination zu bevorzugen ist [415, 446]. Zu den Behandlungsschemata wird auf $>$ Tab. 12 verwiesen.

Im klinischen Stadium I sind die Langzeitergebnisse nach definitiver Radiochemotherapie bei T1-Tumoren mit 5-Jahres-Überlebensraten um $60-70 \%$ besser, als in den höheren Stadien [447, 448]. Auch gibt es bei T1 N0 M0 Tumoren ausreichend Daten zur lokalen Kontrolle und zum Langzeitüberleben nach alleiniger perkutaner Strahlentherapie +/- Brachytherapie. Bei diesen Tumoren wurden lokale Tumorkontrollraten von 45-> $90 \%$ und 5-JahresÜberlebensraten von 38 - 76 \% in mehreren Studien erzielt, sodass bei Kontraindikationen gegen eine begleitende Chemotherapie, die definitive Strahlentherapie bei nicht operablen Patienten eingesetzt werden kann [445, 449].

\begin{tabular}{|l|l|l|}
\hline $\mathbf{8 . 3 2 .}$ & Konsensbasierte Empfehlung & $\begin{array}{l}\text { geprüft } \\
\mathbf{2 0 1 8}\end{array}$ \\
\hline EK & $\begin{array}{l}\text { Bei Patienten mit lokalisiertem Plattenepithelkarzinom } \\
\text { des zervikalen Ösophagus sollte die definitive Radioche- } \\
\text { motherapie gegenüber der primären chirurgischen } \\
\text { Resektion bevorzugt durchgeführt werden. }\end{array}$ \\
\hline $\begin{array}{l}\text { Konsens- } \\
\text { stärke }\end{array}$ & \begin{tabular}{l} 
Starker Konsens (100\%) \\
\hline
\end{tabular} & \\
\hline
\end{tabular}

\section{Hintergrund}

Mit der definitiven Radiochemotherapie werden beim Plattenepithelkarzinom des zervikalen Ösophagus Langzeitüberlebensraten von 17 - 55 \% erreicht $[450,451]$. Die besten Ergebnisse wurden in Serien mit einem hohen Anteil an Patienten im Stadium I 
- Tab. 12 Mögliche Chemotherapieregime bei neoadjuvanter präoperativer Radiochemotherapie [413 - 415].

\begin{tabular}{|c|c|c|c|}
\hline Substanzen & Dosierung & Applikation & Tage \\
\hline \multicolumn{4}{|c|}{ 1. 5-Fluorouracil (5-FU)/Cisplatin } \\
\hline $\begin{array}{l}\text { 5-FU } \\
\text { Cisplatin }\end{array}$ & $\begin{array}{l}1000 \mathrm{mg} / \mathrm{m}^{2} \\
75 \mathrm{mg} / \mathrm{m}^{2}\end{array}$ & $\begin{array}{l}\text { 24h-Infusion } \\
\text { i. v. (60 min) }\end{array}$ & $\begin{array}{l}\text { d1 }-4,29-32 \\
d 1,29\end{array}$ \\
\hline \multicolumn{4}{|c|}{ 2. Carboplatin/Paclitaxel } \\
\hline Carboplatin Paclitaxel & $\begin{array}{l}\text { AUC } 2 \\
50 \mathrm{mg} / \mathrm{m}^{2}\end{array}$ & $\begin{array}{l}\text { i. v. }(60 \mathrm{~min}) \\
\text { i. v. }(60 \mathrm{~min})\end{array}$ & $\begin{array}{l}\text { d 1, 8,15, 22, } 29 \\
\text { d 1, 8,15, 22, } 29\end{array}$ \\
\hline \multicolumn{4}{|l|}{ 3. FOLFOX } \\
\hline $\begin{array}{l}\text { Oxaliplatin Folinsäure } \\
5 \text {-FU } \\
\text { 5-FU }\end{array}$ & $\begin{array}{l}85 \mathrm{mg} / \mathrm{m}^{2} \\
200 \mathrm{mg} / \mathrm{m}^{2} \\
400 \mathrm{mg} / \mathrm{m}^{2} \\
1600 \mathrm{mg} / \mathrm{m}^{2}\end{array}$ & $\begin{array}{l}\text { i.v. } 2 \mathrm{~h} \\
\text { i.v. } 2 \mathrm{~h} \\
\text { i.v. } 10 \mathrm{~min} \\
\text { i.v. } 46 \mathrm{~h}\end{array}$ & $\begin{array}{l}\text { Tag } 1 \\
\text { Tag } 1 \\
\text { Tag } 1 \\
\text { Tag } 1-2 \\
\text { Wiederholung alle } 2 \text { Wo., } 3 \text { Zyklen } \\
\text { während der neoadjuvanten Radioche- } \\
\text { motherapie }\end{array}$ \\
\hline
\end{tabular}

und IIA beobachtet [452]. Die simultane Chemotherapie der definitiven Radiochemotherapie wird bei Plattenepithelkarzinomen des zervikalen Ösophagus wie bei Karzinomen des thorakalen Ösophagus durchgeführt. Die Gesamtstrahlendosis liegt in den größeren retrospektiven Fallserien meist oberhalb von 50 Gy bei 60 - 66 Gy in konventioneller Fraktionierung $[450,451]$ und damit höher als beim thorakalen Ösophagus. Bezüglich der Zielvolumina wird der makroskopische Primärtumor mit cranio-caudalen Sicherheitssäumen von $3,0-4,0 \mathrm{~cm}$ und radialen Sicherheitssäumen von 1,0-1,5 cm, nicht jedoch über intakte anatomische Grenzen hinaus, ins klinische Zielvolumen bis zu einer Basisdosis von 50 Gy einbezogen. Makroskopisch befallene Lymphknoten werden mit einem Saum von 1,5 cm ins klinische Zielvolumen einbezogen [449, 453, 454]. Zur Berücksichtigung von set-up Abweichungen bei der täglichen Einstellung wird um das klinische Zielvolumen (CTV) noch ein Saum von 1,0-1,5 cm in alle Raumrichtungen addiert. Das resultierende Planungszielvolumen umfasst dann den makroskopischen Tumor in der Regel mit einem Saum von 4-5cm kranio-caudal und 1,5-2,0 cm radial. Bei Karzinomen des zervikalen Ösophagus werden meist auch Lymphknotenstationen mit hohem Befallsrisiko, die zervikalen parajugulären Lymphknoten der Level III-IV, die paratrachealen und paraösophagealen Lymphknoten des Halses und im oberen Mediastinum und die medialen supraklavikulären Lymphknoten elektiv bis zu einer Basisdosis von 50 Gy ins Zielvolumen einbezogen [455, 456]. Jedoch ist die Evidenz für eine elektive Mitbestrahlung von klinisch nicht befallenen Lymphknotenstationen gering [457]. Oberhalb von $50 \mathrm{~Gy}$ werden die klinischen Zielvolumina auf ca.1,0 cm um den makroskopischen Tumor reduziert [458].

Vergleichende Untersuchungen zwischen operativem Vorgehen mit neoadjuvanter Radiochemotherapie und der definitiven Radiochemotherapie gibt es nur bei Plattenepithelkarzinomen des thorakalen Ösophagus. Sie zeigten dort keinen Unterschied im Überleben [301]. Beim zervikalen Ösophagus ist die Morbidität der Operation mit und ohne Pharyngolaryngektomie höher als im thorakalen Ösophagus [451, 459, 460]. Daher sollte die Operation nur in spezialisierten Zentren durchgeführt werden. Die 5-JahresÜberlebensraten nach Operation mit oder ohne neoadjuvanter oder adjuvanter Radiochemotherapie betragen in den größeren Serien 14-47\%, ein Bereich der auch durch die Studien zur definitiven Radiochemotherapie abgedeckt wird [451].

\begin{tabular}{|l|l|l|}
\hline $\mathbf{8 . 3 3 .}$ & Evidenzbasierte Empfehlung & $\begin{array}{l}\text { geprüft } \\
\mathbf{2 0 1 8}\end{array}$ \\
\hline $\begin{array}{l}\text { Empfeh- } \\
\text { lungsgrad } \\
\text { A }\end{array}$ & $\begin{array}{l}\text { Bei Patienten mit resektablen Plattenepithelkarzinomen } \\
\text { des intrathorakalen Ösophagus der Kategorie cT3/ cT4 } \\
\text { kann alternativ zur chirurgischen Resektion eine definitive } \\
\text { Radiochemotherapie durchgeführt werden. }\end{array}$ \\
\hline $\begin{array}{l}\text { Level of } \\
\text { Evidence }\end{array}$ & Literatur: $[111,301,428,442,444,449,454,461-464]$ \\
\hline $\begin{array}{l}\text { Konsens- } \\
\text { stärke }\end{array}$ & Starker Konsens (100\%) \\
\hline
\end{tabular}

\section{Hintergrund}

Abweichend von der in > Tab. 4 dargestellten Systematik entspricht die Formulierung mit „kann“ in dieser Empfehlung nicht dem Empfehlungsgrad 0 (Option).

Bei Patienten mit resektablen Plattenepithelkarzinomen des thorakalen Ösophagus der Kategorie cT3 / cT4 ohne hämatogene Fernmetastasen wurden randomisierte Studien zum Vergleich von definitiver Radiochemotherapie mit einer Operation durchgeführt, wobei in der Mehrzahl der Studien eine neoadjuvante Radiochemotherapie in den chirurgischen Armen durchgeführt wurde [301, 428]. Keine der Studien zeigte einen signifikanten Überlebensvorteil im Arm mit Chirurgie. Auch zeigten die Metaanalysen keine Unterschiede bezüglich des Überlebens [301, 428]. Loko-regionale Rezidive waren hingegen nach definitiver Radiochemotherapie häufiger als nach neoadjuvanter Radiochemotherapie und Operation. Die Notwendigkeit zur Bougierung ist nach 
definitiver Radiochemotherapie häufiger als nach neoadjuvanter Radiochemotherapie und Operation. Häufigste Erstrezidive nach neoadjuvanter Radiochemotherapie und Operation waren Fernmetastasen [462]. Die therapiebedingte Letalität war in den chirurgischen Armen höher als nach definitiver Radiochemotherapie. Somit bestehen Unterschiede in der loko-regionalen Effektivität und in der Häufigkeit schwerer Nebenwirkungen, die bei ähnlicher Überlebenschance für die Therapieempfehlung bei dem einzelnen Patienten von Bedeutung sind. Die Ergebnisse der Metaanalysen randomisierter Studien zum Vergleich zwischen definitiver Radiochemotherapie und Operation wurden durch eine große retrospektive Registerstudie mit ähnlicher Fallzahl bestätigt [442].

Bei der definitiven Radiochemotherapie sind als Chemotherapieschemata simultan zur Strahlentherapie Cisplatin und ein Fluoropyrimidin [462, 463, 465], Carboplatin und Paclitaxel [411, 466], Cisplatin und Paclitaxel [467], oder das FOLFOX4 Schema [413] effektiv und tolerabel. Bezüglich der zu applizierenden Gesamtdosis der Strahlentherapie bei der definitiven Radio-Chemotherapie zeigte die Nordamerikanische Intergroup-Studie keinen Überlebensvorteil nach der höheren Gesamtstrahlendosis von 64,8 Gy im Vergleich zur niedrigeren Gesamtdosis von $50,4 \mathrm{~Gy}$ bei konventioneller Fraktionierung [463]. Allerdings kann diese eine Studie wegen gehäufter Protokollverstöße und einer höheren Sterblichkeit im Hochdosisarm in einem Zeitraum, in dem die Therapie in beiden Armen gleich ist, als Hinweis auf ein Ungleichgewicht von Prognosefaktoren kritisiert werden. Seit dieser Studie ist eine Gesamtstrahlendosis von 50 Gy bei simultaner Chemotherapie die Standarddosis für eine definitive Radiochemotherapie in Nordamerika [464]. Die randomisierten Studien zum Vergleich der neoadjuvanten Radiochemotherapie und Operation mit einer definitiven Radiochemotherapie beim Plattenepithelkarzinom des Ösophagus verwendeten jedoch alle Gesamtstrahlendosen von $\geq 60$ Gy $[301,462,468]$. Diese Studien zeigen, dass höhere Gesamtdosen bei definitiver Radiochemotherapie sicher applizierbar sind. Für die Existenz einer Dosis-Effekt-Beziehung für die lokale Tumorkontrolle durch eine definitive Radiochemotherapie sprechen retrospektive Analysen [444]. Eine Gesamtdosis von 50-60 Gy wird auch in den Japanischen und Chinesischen Leitlinien für die definitive Radiochemotherapie empfohlen [449, 454]. Insgesamt spricht die vorhandene Evidenz für eine Gesamtdosis von 50 - 60 Gy in konventioneller Fraktionierung bei der definitiven Radiochemotherapie lokal fortgeschrittener Ösophaguskarzinome, falls die Grenzwerte der Normalgewebetoleranz bei der Strahlentherapie nicht überschritten werden. Bezüglich der Zielvolumina wird der makroskopisch befallene Primärtumor mit cranio-caudalen Sicherheitssäumen von 2,0 $4,0 \mathrm{~cm}$ und radialen Sicherheitssäumen von $1,0-1,5 \mathrm{~cm}$, nicht jedoch über intakte anatomische Grenzen hinaus, ins klinische Zielvolumen bis zu einer Basisdosis von 50 Gy einbezogen. Makroskopisch befallene Lymphknoten werden mit einem Saum 1,0$1,5 \mathrm{~cm}$ ins klinische Zielvolumen einbezogen [449, 454, 464]. Oberhalb von $50 \mathrm{~Gy}$ werden die klinischen Zielvolumina auf ca.1,0 cm um den makroskopischen Tumor reduziert. Die DosisVolumen-Grenzwerte für die Lunge werden in einigen Institutionen bei der Strahlentherapie des Ösophaguskarzinoms niedriger als beim Lungenkarzinom gewählt $[469,470]$. Mit der intensitätsmodulierten Strahlentherapie lässt sich die Belastung des Herzens im Vergleich zur dreidimensional konformalen Strahlentherapie reduzieren [469]. Für die elektive Bestrahlung klinisch nicht befallener Lymphknotenstationen gibt es keine höhergradige Evidenz und daher wird deren Notwendigkeit kontrovers eingeschätzt $[390,442]$.

8.4.6. Vorgehen bei Tumorpersistenz/Lokalrezidiv nach Radiochemotherapie

\begin{tabular}{|l|l|l|}
\hline $\mathbf{8 . 3 4 .}$ & Konsensbasierte Empfehlung & $\begin{array}{l}\text { geprüft } \\
\mathbf{2 0 1 8}\end{array}$ \\
\hline EK & $\begin{array}{l}\text { Bei Tumorpersistenz oder einem Lokalrezidiv ohne Fern- } \\
\text { metastasen nach Radiochemotherapie kann der Versuch } \\
\text { einer Salvage-Operation in kurativer Intention unternom- } \\
\text { men werden. Die sorgfältige Evaluation der Operabilität } \\
\text { und der Resektabilität sollte durch ein in der Ösophagu- } \\
\text { schirurgie erfahrenes Behandlungsteam vorgenommen } \\
\text { werden. }\end{array}$ \\
\hline Konsens- & $\begin{array}{l}\text { Starker Konsens (100\%) } \\
\text { stärke }\end{array}$ & \\
\hline
\end{tabular}

\section{Hintergrund}

Es galt lange als ungeschriebenes Gesetz, dass eine Ösophagusresektion möglichst nicht später als 6 Wochen nach Abschluss einer Radio(chemo)therapie durchgeführt werden sollte, weil durch einsetzende Fibrosierungsvorgänge nicht nur die OP technisch aufwändiger, sondern die postoperative Letalität deutlich ansteigen würde.

Retrospektive Daten großer Zentren aus Italien und den USA haben allerdings gezeigt, dass relevante postoperative Komplikationen und die Letalität nicht signifikant ansteigen, wenn später als 8 Wochen nach Therapie operiert wird [471, 472]. Dies gilt für Plattenpithel- und Adenokarzinome, vorausgesetzt es besteht große Erfahrung des Behandlungsteams.

Diese Erkenntnisse sind Grundlage dafür, das Konzept der Salvage-OP (OP nur für ausgewählte Patienten) zu verfolgen und den Ergebnissen der geplanten OP (OP in der Regel) gegenüberzustellen. Bei der Salvage-OP müssen dabei zwei unterschiedliche Szenarien unterschieden werden: die Ösophagektomie direkt im Anschluss an eine nicht ausreichend wirksame Radiochemotherapie (histologisch nachgewiesene Tumorpersistenz) und die Operation bei histologisch nachgewiesenem, isoliertem Lokalrezidiv nach primär klinisch kompletter Remission durch Radiochemotherapie (Tumorrezidiv).

Zahlreiche Gruppen insbesondere aus Asien haben dazu retrospektive Daten ihrer meist unizentrischen Erfahrungen publiziert [473]. Aus diesen Publikationen kann man ablesen, dass die postoperativen Komplikationen und die Prognose sich deutlich unterscheiden, je nachdem ob es sich um Patienten mit Tumorpersistenz oder Tumorrediziv nach CR handelt. Darüber hinaus ist die R0-Resektion der wichtigste Prognosefaktor [474] und eine Salvage-OP ist nur dann sinnvoll, wenn eine komplette Resektion gelingt. Die Hospital Letalität liegt zwischen 6 und $22 \%$.

Einzelne große Zentren haben mittels sog. „matched pair Analysen" die regelhafte mit der Salvage OP nach Radiochemotherapie 
verglichen [475, 476]. Forscher aus dem MD Anderson Cancer Center (USA) [475] haben 65 Patienten mit einem Adenokarzinom des Ösophagus nach trimodaler Therapie (Chemoradiotherapie + 50 Gy gefolgt von OP) mit 65 Patienten verglichen, die ähnliche Risikofaktoren hatten, aber nach der gleichen Chemoradiotherapie (definitive CRT mit 50 Gy) nur dann eine OP erhielten, wenn eine lokale Tumorpersistenz oder ein lokales Tumorrezidiv auftraten. Das Intervall zwischen Ende der CRT und Salvage-OP betrug median 31 Wochen. Die Rate an R0-Resektionen war nach geplanter OP signifikant höher (99\% vs. $91 \%$ ). Die Komplikationsrate (z. B. Anastomoseninsuffizienz $16,9 \%$ vs. $18,5 \%)$, die Hospital-Letalität $(7,7 \%$ vs. 4,6\%) und die Überlebensrate nach 3 Jahren (55\% vs. $48 \%$ ) unterschieden sich nicht signifikant.

Eine prospektive Studie aus Japan [477] behandelte Patienten mit lokalisiertem Plattenepithelkarzinom des Ösophagus auf dem Boden einer sog. informed decision. Patienten wählten entweder eine Chemoradiotherapie (Cisplatin/5-FU + 60 Gy) gefolgt von OP ( $n=48)$ oder die gleiche Chemoradiotherapie gefolgt von einer Salvage-OP nur bei isoliert lokalem Tumorrest/Tumorprogression ( $n=51$ ). Aus der ersten Gruppe wurden 46/48 Patienten operiert, aus der 2. Gruppe erhielten nur 13/51 Patienten (26\%) eine OP. Die mediane Überlebenszeit (41,2 vs. 52,9 Monate) und die Überlebensrate nach 5 Jahren ( $51 \%$ vs. $76 \%$ ) waren in der Gruppe mit Salvage-OP signifikant höher.

Eine Metaanalyse liegt inzwischen zum Vergleich geplante versus selektive OP nach Chemoradiotherapie vor [302]. Die Metaanalyse schloss 8 vergleichende Studien (in der Regel retrospektiv) mit 954 Patienten unterschiedlicher histologischer Typen des Ösophaguskarzinoms ein. In dieser Analyse war die SalvageOP mit signifikant mehr Komplikationen (z. B. Anastomoseninsuffizienz $14,5 \%$ vs. $24 \%, p=0,005)$ und höherer postoperativer Letalität ( $4 \%$ vs. 9,5\%, p<0,001) verbunden. Die Rate inkompletter Resektionen war nicht unterschiedlich.

Zusammenfassend ist das Konzept der selektiven Salvage-OP nach Chemoradiotherapie durchführbar. Das Vorgehen bietet sich insbesondere für Patienten mit erhöhtem OP-Risiko und/ oder hohem Tumorsitz (suprabifurkal) an. Die Gleichwertigkeit mit einer trimodalen Therapie hinsichtlich der Prognose ist bisher nicht belegt.

\subsubsection{Stellenwert von gezielten Therapien (targeted therapy)}

\begin{tabular}{|l|l|l|}
\hline $\mathbf{8 . 3 5 .}$ & Konsensbasierte Empfehlung & $\begin{array}{l}\text { geprüft } \\
\mathbf{2 0 1 8}\end{array}$ \\
\hline EK & $\begin{array}{l}\text { Antikörper und „small molecules“ sollen in der präope- } \\
\text { rativen Therapie nicht eingesetzt werden. }\end{array}$ \\
\hline $\begin{array}{l}\text { Konsens- } \\
\text { stärke }\end{array}$ & Konsens (90\%) \\
\hline
\end{tabular}

\section{Hintergrund}

In der palliativen Behandlung fortgeschrittener Adenokarzinome des Magens oder gastroösophagogastralen Übergangs wurden Phase-III-Daten publiziert, die einen signifikanten Überlebensvorteil für Patienten mit HER2-positiven Tumoren nachwei- sen, wenn sie zur Chemotherapie aus Cisplatin und einem Fluoropyrimid den HER2 Antikörper Trastuzumab erhielten [228]. Es ist aber trotz erster kleiner Phase-II-Studien unklar, ob dieser Antikörper auch die Ergebnisse einer präoperativen Therapie bei lokalisierten, HER2-positiven Karzinomen verbessert [18, 404].

Bei Plattenepithelkarzinomen des Ösopahgus gibt es keine klaren positiven Ergebnisse von zielgerichteten Antikörpern. Der Einsatz zielgerichteter Substanzen ist daher außerhalb klinischer Studien nicht indiziert. Die Ergebnisse laufender randomisierter Studien müssen abgewartet werden.

\subsubsection{Stellenwert der postoperativen adjuvanten Radiotherapie oder Radiochemotherapie}

\begin{tabular}{|l|l|l|}
\hline $\mathbf{8 . 3 6 .}$ & Evidenzbasierte Empfehlung & $\begin{array}{l}\text { geprüft } \\
\mathbf{2 0 1 8}\end{array}$ \\
\hline $\begin{array}{l}\text { Empfeh- } \\
\text { lungsgrad } \\
\mathbf{A}\end{array}$ & $\begin{array}{l}\text { Nach R0-Resektion eines Plattenepithelkarzinoms soll } \\
\text { eine adjuvante Radiotherapie oder Radiochemotherapie } \\
\text { nicht durchgeführt werden. }\end{array}$ \\
\hline $\begin{array}{l}\text { Level of } \\
\text { Evidence } \\
\mathbf{4} \text { (CRT) } \\
\mathbf{1 a}(\mathbf{R T})\end{array}$ & Literatur: $[478,479]$ \\
\hline $\begin{array}{l}\text { Konsens- } \\
\text { stärke }\end{array}$ & Starker Konsens $(96 \%)$ \\
\hline
\end{tabular}

\section{Hintergrund}

Die randomisierten Studien zur Effektivität der adjuvanten postoperativen alleinigen Strahlentherapie beim Plattenepithelkarzinom des Ösophagus nach kompletter Resektion fassten Malthaner et al. [386] in ihrer Metaanalyse zusammen. Die postoperative Strahlentherapie war mit keinem signifikanten Überlebensvorteil verknüpft und es bestand auch kein Trend zu einem besseren Überleben mit postoperativer Strahlentherapie. Alle Studien wurden vor mehr als 15 Jahren abgeschlossen. Aus diesen Studien gibt es Hinweise, dass durch die Strahlentherapie das loko-regionäre Rezidivrisiko gesenkt wird, dass die Strahlentherapie zumindest mit den damaligen Methoden aber auch mit einer zusätzlichen Morbidität verknüpft sein kann [379]. In neueren Übersichten wurden keine weiteren randomisierten Studien zur adjuvanten Strahlentherapie beim Plattenepithelkarzinom des Ösophagus identifiziert $[479,480]$.

Zur Effektivität der adjuvanten Radiochemotherapie beim Plattenepithelkarzinom gibt es zwei randomisierte Studien. Die Studie von Thallinger mit 45 randomisierten Patienten [479] verglich eine postoperative Radiotherapie mit 50 Gy in konventioneller Fraktionierung und simultanem Cisplatin/5-FU mit einer alleinigen postoperativen Chemotherapie. Ein Trend zu einem Überlebensvorteil mit der postoperativen Radiochemotherapie wurde nicht gefunden. In der dreiarmigen Studie aus Nanjing wurden insgesamt 238 Patienten mit Plattenepithelkarzinom des Ösophagus randomisiert in die Arme neoadjuvante Radiochemotherapie und Operation, alleinige Operation und Operation mit postoperativer Radiochemotherapie [481, 482]. Da die Patienten präoperativ randomisiert wurden, handelt es sich hier nicht um 
eine reine Überprüfung der adjuvanten Therapie nach kompletter Resektion. Die Arme mit prä- oder postoperativer Radiochemotherapie zeigten vergleichbare Überlebensdaten, die signifikant besser war als im Arm mit alleiniger Chirurgie [481]. Zheng [478] analysierten in ihrer Metaanalyse noch 5 weitere vergleichende Studien zur postoperativen Radiochemotherapie, die aber alle nicht randomisiert waren. Diese wiesen ebenfalls auf eine Aktivität der postoperativen Radiochemotherapie hin. Insgesamt wird die Evidenzlage für eine Empfehlung einer adjuvanten postoperativen Radiochemotherapie als Standardoption nicht als ausreichend erachtet. Die größere Evidenz spricht für den Einsatz der präoperativen Radiochemotherapie bei T2-T4 Tumoren ohne Fernmetastasen.

\begin{tabular}{|l|l|l|}
\hline $\begin{array}{l}\text { 8.37. } \\
\text { geprüft }\end{array}$ & \begin{tabular}{l}
$\mathbf{2 0 1 8}$ \\
\hline $\begin{array}{l}\text { Empfeh- } \\
\text { lungsgrad } \\
\mathbf{0}\end{array}$
\end{tabular} & $\begin{array}{l}\text { Nach R0-Resektion eines Adenokarzinoms des ösophago- } \\
\text { gastralen Übergangs kann bei erhöhtem Lokalrezidivrisiko } \\
\text { eine adjuvante Radiochemotherapie bei nicht neoadju- } \\
\text { vant behandelten Patienten durchgeführt werden. }\end{array}$ \\
\hline $\begin{array}{l}\text { Level of } \\
\text { Evidence } \\
\mathbf{1 b}\end{array}$ & Literatur: $[18,483-485]$ \\
\hline $\begin{array}{l}\text { Konsens- } \\
\text { stärke }\end{array}$ & Starker Konsens $(100 \%)$ \\
\hline
\end{tabular}

\section{Hintergrund}

Die Evidenz für einen Nutzen einer präoperativen Radiochemotherapie oder perioperativen Chemotherapie ist höher als der für eine postoperative Radiochemotherapie, sodass eine präoperative Behandlung eindeutig präferiert werden soll (siehe 8.4.2). Falls aufgrund eines präoperativen Unterstagings keine präoperative Therapie erfolgt ist, kann eine postoperative Radiochemotherapie durchgeführt werden. Vor Beginn einer adjuvanten Radiochemotherapie ist das Fortbestehen der kurativen Therapieintention zu überprüfen und Fernmetastasen sind auszuschließen. Im Falle von Fernmetastasen ist ein Radiochemotherapiekonzept nicht mehr sinnvoll. Im Falle der Fernmetastasierung ist die Umstellung auf eine palliative Therapie sinnvoll (siehe Kapitel 9).

Der Effekt einer postoperativen Radiochemotherapie wurde beim Adenokarzinom des Magens in 8 randomisierten Studien im Vergleich zur alleinigen Operation oder Operation mit postoperativer Chemotherapie untersucht [483]. Die Metaanalyse dieser Studien zeigt ein verbessertes progressionsfreies Überleben (HR 0,66: $95 \% \mathrm{KI}$ 0,55-0,78) und Gesamtüberleben (HR 0,75; $95 \%$ KI 0,63 - 0,89) [483] mit postoperativer Radiochemotherapie im Vergleich zur alleinigen Operation und ein verbessertes progressionsfreies Überleben (HR 0,77; $95 \%$ KI 0,65-0,91) im Vergleich zur Operation mit postoperativer Chemotherapie. Der Anteil von Karzinomen des ösophagogastralen Übergangs und der Kardia lag in den Studien bei 6 - 23 \% [484 - 487]. Daher kann, wie beim Magenkarzinom, auch für Adenokarzinome des ösophago-gastralen Übergangs eine postoperative Radiochemotherapie durchgeführt werden, wenn ein erhöhtes Lokalrezidivrisiko angenommen wird. Dies kann bei multiplen befallenen Lymphknoten oder bei eingeschränkter Lymphadenektomie der Fall sein [485, 486]. Bei der adjuvanten postoperativen Radiochemotherapie wird eine Gesamtdosis von 45 - 50,4 Gy in konventioneller Fraktionierung mit 1,8-2,0 Gy pro Fraktion, 5 Fraktionen pro Woche, appliziert. Die simultane Chemotherapie kann z. B. mit Capecetabine wie in der Artist Studie [485] durchgeführt werden. Bezüglich des originalen Intergroup 0116-Schemas wurden von den Verfassern der NCCN-Leitlinie zum Ösophaguskarzinom Sicherheitsbedenken geäußert.

Die Empfehlungen bezüglich einer alleinigen adjuvanten Chemotherapie bei Adenokarzinomen des gastro-ösophagealen Übergangs orientieren sich an der S3-Leitlinie zum Magenkarzinom [18]. Die beim Magenkarzinom durchgeführten Metaanalysen unterscheiden nicht zwischen dem eigentlichen Magenkarzinom und dem Adenokarzinom des gastroösophagealen Übergangs. Nach primärer R0-Resektion (ohne präoperative Chemotherapie) sollte keine adjuvante Chemotherapie durchgeführt werden.

Diese relative Negativempfehlung beruht auf dem Wunsch, bei Adenokarzinomen des ösophagogastralen Übergangs eine positive Präferenz der perioperativen Chemotherapie bzw. präoperativen Radiochemotherapie als primäre Behandlungsstrategie zu etablieren. Eine alleinige adjuvante Chemotherapie wird nicht als primäres Therapiekonzept empfohlen, da 1) eine alleinige adjuvante Therapie aufgrund des postoperativen Allgemeinzustandes bei viel weniger Patienten durchführbar ist [390] und 2) der Nutzen eines perioperativen Therapiekonzeptes durch positive Einzelstudien besser belegt ist (siehe 8.4.2) und 3) die Überlebensverbesserung durch eine perioperative Therapie größer zu sein scheint, als durch eine alleinige adjuvante Therapie (13,8\% vs. $5,8 \%$; [488].

Sollte jedoch bei Patienten durch ein unzureichendes Staging oder Notoperation kein präoperatives Therapieverfahren eingeleitet worden sein, kann bei Patienten mit primär lokal fortgeschrittenem Tumorstadium, v. a. mit positiven Lymphknoten, die adjuvante Chemotherapie nach der vorliegenden Evidenz erwogen und angeboten werden [18].

Im Jahr 2010 veröffentlichte die GASTRIC Group [488] eine Metaanalyse auf dem Boden individueller Patientendaten aus insgesamt 3838 Patienten aus 17 Studien. Diese Studie weist einen Vorteil für die adjuvante Chemotherapie gegenüber der alleinigen Operation auf. Die Hazard Ratio für das Gesamtüberleben beträgt 0,82 (95\% KI 0,76-0,90, p<0,001, absolut 5,8\% nach 5 Jahren). In dieser Analyse zeigt sich auch bei europäischen Patienten ein Vorteil durch eine adjuvante Therapie [404].

\begin{tabular}{|l|l|l|}
\hline 8.38. & Konsensbasiertes Statement & $\begin{array}{l}\text { geprüft } \\
\mathbf{2 0 1 8}\end{array}$ \\
\hline EK & $\begin{array}{l}\text { Die Nachsorge bei operativ oder radiochemotherapeu- } \\
\text { tisch behandelten Patienten mit Ösophaguskarzinom } \\
\text { erfolgt symptomorientiert, um die Lebensqualität beein- } \\
\text { flussende Funktionsstörungen zu erfassen. Eine symp- } \\
\text { tomorientierte Anamneseerhebung und körperliche } \\
\text { Untersuchung sind grundsätzlicher Bestandteil der }\end{array}$ \\
\hline Konsens- & Stachsorge. \\
\hline stärke & Stansens (100\%) \\
\hline
\end{tabular}




\section{Hintergrund}

Es gibt Gründe für eine symptomorientierte Nachsorge bei Patienten mit einem ösophagogastralen Karzinom:

- um Funktionsstörungen zu entdecken in Verbindung mit einem Rezidiv oder als benigne Komplikationen der Behandlung

- um den Ernährungszustand zu beurteilen und ggf. Ernährungsprobleme zu handhaben

- um für den Patienten und die Familie psychische Unterstützung anzubieten, mit angemessenen medizinischen Maßnahmen in Verbindung mit der Palliativmedizin

Im Rahmen der symptomorientierten Nachsorge ist eine Anamnese, eine körperliche Untersuchung einschließlich Gewicht und eine Blutbild-Bestimmung (ggf. ergänzt um Eisen, Ferritin, TransferrinSättigung) durchzuführen. Diese Untersuchungen erfolgen zu Beginn kurzfristiger, um Komplikationen schneller zu entdecken und eine Balance des Nahrungshaushaltes zu sichern [489, 490]

\begin{tabular}{|l|l|l|}
\hline 8.39. & Konsensbasierte Empfehlung & $\begin{array}{l}\text { geprüft } \\
\mathbf{2 0 1 8}\end{array}$ \\
\hline EK & $\begin{array}{l}\text { In den ersten 6 Monaten sollten regelmäßige Verlaufs- } \\
\text { kontrollen des Ernährungsstatus einschließlich Diätbera- } \\
\text { tung erfolgen. Die Supplementierung der oralen Energie- } \\
\text { zufuhr mit Trinklösung oder sogar Sondenernährung über } \\
\text { eine zunächst belassene Feinnadelkatheterjejunostomie } \\
\text { kann empfohlen werden. }\end{array}$ \\
\hline $\begin{array}{l}\text { Konsens- } \\
\text { stärke }\end{array}$ & \begin{tabular}{l} 
Starker Konsens (100\%) \\
\hline
\end{tabular} \\
\hline
\end{tabular}

\section{Hintergrund}

Selbst bei völlig unkompliziertem postoperativem Verlauf wird bei den meisten Patienten die orale Kalorienzufuhr für eine längere Periode oftmals erheblich unter dem tatsächlichen Energiebedarf liegen. So kann nach Ösophagusresektion bei $30 \%$ der Patienten ein Gewichtsverlust von mehr als $15 \%$ innerhalb von 6 Monaten erwartet werden [491].

Eine prospektive Kohortenstudie hat nur bei $10 \%$ der Patienten mit Ösophagusresektion ( $n=96)$, die eine Rekonstruktion als Magenhochzug erhielten, eine den allgemeinen Empfehlungen folgende ausreichende Zufuhr an Mikronährstoffen gezeigt [492]. Die Ursachen entsprechen denen der bariatrisch durchgeführten Magenschlauchbildung als „Sleeve“ Gastrektomie: Verlust an Appetit, verminderte enterale Toleranz, evtl. mit Dumping-Syndrom, Meteorismus und Diarrhoe.

Im Rahmen der der Leitlinie entsprechenden früh postoperativen Ernährungstherapie [235] wird eine Ernährungsberatung dringend empfohlen und von den meisten Patienten sehr gerne angenommen.

Sofern bei der Operation eine Feinnadelkatheterjejunostomie (FKJ) implantiert wurde, kann es von Vorteil sein, diese nicht bereits bei der Entlassung aus dem Krankenhaus zu entfernen. Wenn notwendig, kann eine supplementierende enterale Ernährung über die FKJ z. B. mit $500-1000 \mathrm{kcal} /$ Tag über Nacht über mehrere Monate erfolgen. Eine entsprechende Unterweisung des Patienten und seiner Familie ermöglicht in den meisten Fällen die Versorgung ohne Einbindung eines Pflegedienstes. Auch wenn ein weiterer Gewichtsverlust häufig unvermeidlich ist, kann doch zumindest eine Abschwächung erwartet werden [233, 493].

Eine Verlaufskontrolle des Ernährungsstatus kann mit der Beobachtung des BMI leicht durchgeführt werden. Jedoch ist der BMI nicht sensitiv für Unterschiede in der Körperzusammensetzung. Die Bioelektrische Impedanz Analyse (BIA) ist eine leicht durchführbare nicht-invasive Methode, welche auch bei ambulanten Patienten ohne Belastung durchgeführt werden kann. Der intraindividuelle Verlauf kann in einem Drei-Kompartiment-Model (Extrazellulärmasse, Körperzellmasse und Fettmasse) dargestellt und beobachtet werden. Von der Körperimpedanz sind das Verhältnis der Extrazellulärmasse zur Körperzellmasse und der Phasenwinkel einfach verfügbare Werte, welche zuverlässige und valide Informationen über den Zellgehalt des Körpers ermöglichen. Idealerweise wird die erste Untersuchung bereits bei Diagnosestellung durchgeführt.

\begin{tabular}{|l|l|l|}
\hline 8.40. & Konsensbasierte Empfehlung & \multicolumn{1}{c|}{$\mathbf{2 0 1 8}$} \\
\hline EK & $\begin{array}{l}\text { Patienten mit Ösophaguskarzinom sollten - im Rahmen } \\
\text { ihrer Möglichkeiten - zu körperlicher Aktivität motiviert } \\
\text { werden. } \\
\text { Nach Abschluss der Primärtherapie sollte allen rehabilita- } \\
\text { tionsfähigen Patienten eine Anschlussheilbehandlung an- } \\
\text { geboten werden. Die rehabilitative Therapie soll medizi- } \\
\text { nische, pflegerische, edukative, trainierende und } \\
\text { psychosoziale Maßnahmen umfassen, die dem individu- } \\
\text { ellen Rehabilitationsbedarf angepasst werden. } \\
\text { Zur Reduzierung des durch die Tumorerkrankung oder die } \\
\text { Tumortherapie bedingten Fatigue-Syndroms sollte ein } \\
\text { sich an der individuellen Belastungsfähigkeit orientieren- } \\
\text { des Ausdauertraining durchgeführt werden. }\end{array}$ \\
\hline Konsens (86\%) \\
\hline Konsens- & Kärke & \\
\hline
\end{tabular}

\section{Hintergrund}

Um die Muskelmasse der Patienten mit Ösophaguskarzinom zu erhalten, sollten ein leichtes Ausdauer- und ein spezielles Muskelaufbautraining empfohlen werden. Es gibt keine spezifischen Studien zu Patienten mit Ösophaguskarzinom. In einer Cochrane Übersichtsarbeit [494] haben die Autoren insgesamt 56 Studien mit 4826 Krebspatienten eingeschlossen. In 36 Studien wurden Teilnehmer während einer laufenden Therapie untersucht, in 10 Studien während der Therapie und danach und in 10 Studien vor Beginn der Therapie. Die Übungsgruppe wies eine signifikante Reduktion von Angst, Depression, Fatigue und Schlafstörungen nach der Bewegungstherapie auf. Ebenso war die körperliche Leistungsfähigkeit verbessert, die soziale Befähigung und Teilhabe wie auch die Lebensqualität. Darüber hinaus untermauern auch neuere Übersichtsarbeiten und Studien [495] bei Patienten mit Lungenkarzinomen, dass eine Bewegungstherapie zur Verbesserung von Ausdauer und Erhalt der Muskelmasse beitragen können.

Patienten mit Ösophaguskarzinom leiden häufig unter einem Gewichtsverlust und einem Erschöpfungssyndrom mit körperlich 
eingeschränkter Leistungsfähigkeit. Daher sollte ein spezielles Rehabilitationsprogramm zur Verbesserung der Ernährungssituation und der kardio-respiratorischen Ausdauer angeboten werden [496]. Auftrag der Rehabilitation ist die möglichst weitgehende Beseitigung - zumindest aber Kompensation - Tumor- oder therapiebedingter Folgen sowie die Hilfestellung bei der Akzeptanz verbleibender Behinderungen mit dem Ziel einer selbstbestimmten Teilhabe am gesellschaftlichen Leben. Für die rehabilitative Maßnahme sind ausgewiesene Reha-Zentren bzw. Kliniken mit gastrointestinaler und onkologischer Expertise zu bevorzugen, die den Standards des Qualitätssicherungsverfahrens der Deutschen Rentenversicherung entsprechen und somit auch Elemente zur Motivation zu mehr Sport und Bewegung beinhalten [497].

Ziel jeder Rehabilitation sind Sicherung und erforderlichenfalls Verbesserung der Lebensqualität des Betroffenen, wobei die Notwendigkeit dieser Maßnahmen individuell einzuschätzen ist. Rehabilitation ist vom Gesetzgeber als sozialer Anspruch definiert (SGB I, § 19). Art und Umfang der erforderlichen Leistungen werden im SGB I (§29), SGB V (Krankenversicherung), SGB VI (Rentenversicherung), SGB III (Arbeitsforderung), ferner im RehAnglG und im SGB IX konkretisiert. Der Rehabilitationsbedarf nach Behandlung von Ösophaguskarzinomen ist äußerst variabel und im Wesentlichen abhängig von Art und Ausmaß des operativen Vorgehens sowie der Therapiefolgen. Rehabilitationsverfahren sollten bei definiertem Rehabilitationsbedarf und individueller Rehabilitationsfähigkeit möglichst im Anschluss an die Primärtherapie stattfinden.

Zum Stellenwert rehabilitativer Maßnahmen bei Patienten mit Ösophaguskarzinom liegt keine auswertbare Literatur vor. In einer Cochrane Übersichtsarbeit [497] haben die Autoren insgesamt 40 randomisierte und kontrollierte klinische Studien mit $3694 \mathrm{~Pa}$ tienten und unterschiedlichen Krebsarten nach abgeschlossener Primärtherapie eingeschlossen. Im Vergleich zu den Kontrollgruppen zeigte sich ein positiver Effekt nach Abschluss des Bewegungsprogramms bezüglich Angst, Müdigkeit, Depression, Sexualität, Schlafstörungen, Schmerzen, Lebensqualität und der sozialen Rolle. Zur Reduzierung des durch die Tumorerkrankung oder therapiebedingten Fatigue-Syndroms sollte ein sich an der individuellen Belastungsfähigkeit orientierendes Ausdauertraining durchgeführt werden.

\section{Palliativtherapie}

bearbeitet durch Pech/Vanhoefer

\subsection{Palliative Chemotherapie: Erstlinientherapie}

\begin{tabular}{|l|l|l|}
\hline $\begin{array}{l}\text { 9.1. } \\
\text { Empfeh- } \\
\text { lungsgrad }\end{array}$ & $\begin{array}{l}\text { geprüft } \\
\mathbf{2 0 1 8}\end{array}$ \\
\hline $\mathbf{A}$ & $\begin{array}{l}\text { Patienten mit einem metastasierten oder lokal fortge- } \\
\text { schrittenen, nicht kurativ behandelbaren Adenokarzinom } \\
\text { des Ösophagus soll eine systemische Chemotherapie } \\
\text { angeboten werden. Therapieziel ist die Verlängerung der } \\
\text { Überlebenszeit und der Erhalt der Lebensqualität. }\end{array}$ \\
\hline
\end{tabular}

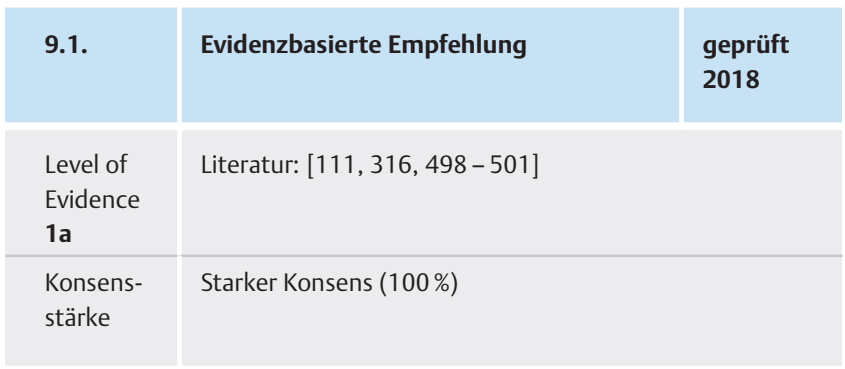

\begin{tabular}{|l|l|l|}
\hline $\mathbf{9 . 2 .}$ & Evidenzbasierte Empfehlung & $\begin{array}{l}\text { geprüft } \\
\mathbf{2 0 1 8}\end{array}$ \\
\hline $\begin{array}{l}\text { Empfeh- } \\
\text { lungsgrad } \\
\text { A }\end{array}$ & $\begin{array}{l}\text { Bei negativem HER2-Status soll hierbei eine Platin } \\
\text { (Oxaliplatin oder Cisplatin)- und Fluoropyrimidin-haltige } \\
\text { Zwei- oder Dreifachkombination eingesetzt werden. }\end{array}$ \\
\hline $\begin{array}{l}\text { Level of } \\
\text { Evidence } \\
\mathbf{1 a}\end{array}$ & \begin{tabular}{l} 
Literatur: [316, $498-505]$ \\
\hline Konsens- \\
stärke
\end{tabular} & Starker Konsens (100\%) \\
\hline
\end{tabular}

\begin{tabular}{|l|l|l|}
\hline 9.3. & Konsensbasierte Empfehlung & $\begin{array}{l}\text { geprüft } \\
\mathbf{2 0 1 8}\end{array}$ \\
\hline EK & $\begin{array}{l}\text { Vor Einleitung einer systemischen palliativen Chemothe- } \\
\text { rapie soll der HER2-Status als prädiktiver Faktor für eine } \\
\text { Therapie mit Trastuzumab bestimmt werden. }\end{array}$ \\
\hline $\begin{array}{l}\text { Konsens- } \\
\text { stärke }\end{array}$ & \begin{tabular}{l} 
Starker Konsens (95\%) \\
\hline
\end{tabular}
\end{tabular}

\section{Hintergrund}

In mehreren randomisierten Phase-III-Studien für das Magenkarzinom stellte die Subgruppe der Adenokarzinome des gastroösophagealen Überganges und der distalen Adenokarzinome des Ösophagus einen erheblichen Anteil der Studienpopulation dar [498 - 502, 506]. So konnte mit einer Platin- und Fluoropyrimidin-basierten Kombinationschemotherapie mit Docetaxel oder Epirubicin eine signifikante Verbesserung hinsichtlich des Überlebens, der Zeit bis zur Tumorprogression und ein Vorteil in der Lebensqualität gegenüber älteren Chemotherapie-Protokollen (FUP, FAMTX) nachgewiesen werden (DCF vs. FUP: Mediane Überlebenszeit 9,2 Monate vs. 8,6 Monate [p=0,02] und progressionsfreies Überleben 5,6 Monate vs. 3,7 Monate [p<0,001] sowie ECF vs. FAMTX: Mediane Überlebenszeit 8,9 Monate vs. 5,7 Monate $[p=0,0009]$ und FFS 7,4 Monate vs. 3,4 Monate $[p=0,00006])$ [499, 501].

Patienten mit negativem HER2-Status soll daher eine Platinund Fluoropyrimidin-haltige Zwei- oder Dreifachkombination angeboten werden. Hierbei kommen u. a. folgende Kombinationen in Betracht: S-1/Cisplatin oder Capecitabin/Cisplatin [XP], infusionales 5-Fluorouracil, Folinsäure und Cisliplatin [PLF], Epirubicin, 
Cisplatin, Capecitabin [ECX], Epirubicin, Oxaliplatin, Capecitabin [EOX], Epirubicin, Cisplatin, infusionales 5-Fluorouracil [ECF], Docetaxel, Cisplatin, infusionales 5-Fluorouracil [DCF], infusionales 5-Fluorouracil/Folinsäure und Oxaliplatin (FLO) oder die Kombination aus 5-Fluorouracil (infusional), Folinsäure, Oxaliplatin und Docetaxel (FLOT-Regime) [53, 111, 404, 498-508].

Bei der Auswahl der Therapieregime sind Allgemeinzustand, Alter, Begleiterkrankungen, Toxizitäten der Therapie und die individuelle Situation des Patienten zu berücksichtigen. Ist eine Docetaxel-basierte Dreifachkombination indiziert, sollten modifizierte Schemata dem klassischen DCF-Regime vorgezogen werden, da das DCF-Regime mit einer erhöhten Toxizität assoziiert ist. In mehreren zum Teil randomisierten Phase-II-Studien konnte gezeigt werden, dass u. a. die Kombination aus 5-Fluorouracil (infusional), Folinsäure, Oxaliplatin und Docetaxel (FLOT-Regime) eine dem DCF-Regime vergleichbare Aktivität bei günstigerem Nebenwirkungsprofil aufweist [507].

Für ältere Patienten konnte in mehreren klinischen Studien gezeigt werden, dass die Kombination aus Oxaliplatin mit einem Fluoropyrimidin (5-Fluorouracil oder Capecitabin) hinsichtlich der Nebenwirkungen durchgeführt werden kann. Das mediane Alter der Patienten lag in diesen Studien zwischen 70 - 77 Jahren. Das mediane Überleben betrug 9,5 bis 11,7 Monate [509-512].

Patienten mit positivem HER2-Status soll eine Therapie mit Trastuzumab und einer Platin-basierten Kombination mit einem Fluoropyrimidin [Capecitabin oder infusionales 5-Fluorouracil) angeboten werden (siehe Empfehlung 6.24.).

Eine palliative Chemotherapie sollte zum frühestmöglichen Zeitpunkt der Diagnosestellung der fortgeschrittenen Erkrankung eingeleitet werden.

\begin{tabular}{|c|c|c|}
\hline 9.4 . & Konsensbasierte Empfehlung & $\begin{array}{l}\text { geprüft } \\
2018\end{array}$ \\
\hline EK & \multicolumn{2}{|c|}{$\begin{array}{l}\text { Patienten mit einem metastasierten oder lokal fortge- } \\
\text { schrittenen, nicht kurativ behandelbaren Plattenepithel- } \\
\text { karzinom des Ösophagus kann eine palliative systemische } \\
\text { Chemotherapie angeboten werden. Therapieziel ist der } \\
\text { Erhalt der Lebensqualität. } \\
\text { Hierbei kann eine Kombinationstherapie aus Cisplatin und } \\
\text { einem Fluoropyrimidin eingesetzt werden. Ein lebensver- } \\
\text { längernder Effekt der systemischen palliativen Chemo- } \\
\text { therapie ist für das Plattenepithelkarzinom des Ösopha- } \\
\text { gus nicht gesichert. }\end{array}$} \\
\hline $\begin{array}{l}\text { Konsens- } \\
\text { stärke }\end{array}$ & Starker Konsens $(100 \%)$ & \\
\hline
\end{tabular}

\section{Hintergrund}

Patienten mit einem metastasierten oder lokal fortgeschrittenem (nicht kurativ behandelbarem Plattenepithelkarzinom des Ösophagus) kann eine systemische palliative Chemotherapie mit dem Ziel einer Erhaltung der Lebensqualität angeboten werden. Ein klinisch relevanter lebensverlängernder Effekt der systemischen palliativen Chemotherapie ist für das Plattenepithelkarzinom des Ösophagus nicht gesichert. Die Datenlage ist hinsichtlich randomisierter klinischer Studien sehr begrenzt und bezieht sich oft nur auf eine Subpopulation von Patienten [53, 111, 127, 498, 513, 514].
In den publizierten klinischen Studien wurde häufig eine Kombinationstherapie von Cisplatin mit einem Fluoropyrimidin (infusionales 5-Fluorouracil oder Capecitabin) eingesetzt. In anderen Studien wurden Platin-basierte Kombinationen u. a. mit Taxanen untersucht.

In der aktuellen Literatur gibt es nur wenige Publikationen, die sich mit der chirurgischen Therapie des metastasierten bzw. lokal rezidivierten Ösophaguskarzinoms beschäftigen. Während randomisierte Studien bzw. Kohortenstudien nicht vorliegen, liegen einige Fallserien bzw. Case-Reports vor, die die Wertigkeit einer chirurgischen Therapie in diesem onkologischen Setting evaluiert haben [53, 302, 475, 515-521]. Im Rahmen der chirurgischen Therapie des metastasierten Ösophaguskarzinoms beschäftigen sich die bisher vorliegenden Publikationen vor allem mit der Versorgung von metachronen Lungen- bzw. Lebermetastasen [499-501, 506]. Dabei lassen die Studien vermuten, dass die operative Versorgung einer metachronen, solitären Lungenmetastase im Rahmen einer individuellen Therapieplanung durchgeführt werden kann, jedoch bei vorliegenden metachronen Lebermetastasen bzw. multilokolären Lungenmetastasen eine chirurgische Versorgung eher nicht durchgeführt werden sollte. So zeigten Ichida et al. [518] erst kürzlich in 26 Patienten mit metachronen Leber- bzw. 27 Patienten mit Lungenmetastasen, die sich zuvor einer Ösophagektomie unterzogen, dass zwar Patienten mit solitären pulmonalen Metastasen einen Prognosevorteil haben können, aber vor allem für Patienten mit Lebermetastasen eher kein relevanter Benefit besteht.

Bei Patienten mit einem lokal rezidivierten Ösophaguskarzinom haben die bisherigen Analysen fast ausschließlich Patienten nach definitiver Radiochemotherapie analysiert, die sich einer sogenannten Salvage-Ösophagektomie unterzogen [53, 302, 475, 519-521].

Dabei kann man zusammenfassen, dass die meisten Studien eine relevante Erhöhung der Morbidität bzw. Mortalität für diese Patientengruppe aufweist und somit nur im Rahmen einer individuellen Therapieplanung eine solche operative Versorgung durchgeführt werden sollte.

Die von der British Association of Surgical Oncology aus dem Jahre 2011 vorliegenden Leitlinien empfehlen ein individuelles therapeutisches Vorgehen bei Lokalrezidiv nach definitiver Radiochemotherapie, jedoch wird ein chirurgisches Vorgehen bei solitären Fernmetastasen von Lunge bzw. Leber nicht diskutiert [53].

\subsection{Palliative Chemotherapie: Zweitlinientherapie}

\begin{tabular}{|l|l|l|}
\hline 9.5. & Konsensbasierte Empfehlung & $\begin{array}{l}\text { geprüft } \\
\mathbf{2 0 1 8}\end{array}$ \\
\hline EK & $\begin{array}{l}\text { Bei Patienten mit einem metastasierten oder lokal fort- } \\
\text { geschrittenen, nicht kurativ behandelbaren Adenokarzi- } \\
\text { nom des Ösophagus und ausreichendem Allgemeinzu- } \\
\text { stand sollte eine systemische Zweitlinientherapie } \\
\text { durchgeführt werden. }\end{array}$ \\
\hline $\begin{array}{l}\text { Konsens- } \\
\text { stärke }\end{array}$ & \begin{tabular}{l} 
Starker Konsens (100\%) \\
\hline
\end{tabular} \\
\hline
\end{tabular}




\section{Hintergrund}

Das Behandlungsschema richtet sich nach der jeweiligen Vortherapie. Therapieziel ist die Verlängerung der Überlebenszeit, der Zeit bis zur Tumorprogression und der Erhalt der Lebensqualität.

Es gibt inzwischen mehrere Phase-III-Studien, die eine Verlängerung des Gesamtüberlebens und zum Teil Erhalt der Lebensqualität unter Zweitlinientherapie zeigen. Dies gilt für die folgenden zytotoxischen Einzelsubstanzen Irinotecan, Paclitaxel, und Docetaxel [522 - 524] sowie neuerdings für den VEGFR2-Antikörper Ramucirumab alleine (kaum Nebenwirkungen) oder in Kombination mit Paclitaxel [525, 526] (siehe auch Hintergrundtext zu Empfehlung 9.7.).

Durch die gute Datenlage ist die Empfehlung mit „sollte“ gerechtfertigt. Die Wortwahl „systemische Zweitlinientherapie“ wird anstelle von „Zweit-Chemotherapie“ angewandt weil Ramucirumab keine Chemotherapie ist. Irinotecan, Paclitaxel, und Docetaxel sind als Zweit-Chemotherapie des Ösophaguskarzinoms nicht zugelassen.

\begin{tabular}{|l|l|l|}
\hline 9.6. & Konsensbasierte Empfehlung & $\begin{array}{l}\text { geprüft } \\
\mathbf{2 0 1 8}\end{array}$ \\
\hline EK & $\begin{array}{l}\text { Bei Patienten mit einem metastasierten oder lokal fort- } \\
\text { geschrittenen, nicht kurativ behandelbaren Plattenepi- } \\
\text { thelkarzinom des Ösophagus und ausreichenden Allge- } \\
\text { meinzustand kann eine Zweitlinientherapie erwogen } \\
\text { werden }\end{array}$ \\
\hline $\begin{array}{l}\text { Konsens- } \\
\text { stärke }\end{array}$ & \begin{tabular}{l} 
Starker Konsens $(100 \%)$ \\
\hline
\end{tabular}
\end{tabular}

\section{Hintergrund}

Es gibt keine belastbaren Daten, die eine Wirksamkeit einer Zweit-Chemotherapie beim Plattenepithelkarzinom des Ösophagus zeigen. Es existieren kleine Phase-II-Studien mit Substanzen wie Taxane, Platinderivate und Irinotecan, aber auch solche mit älteren Substanzen wie Mitomycin C [527]. Daher wurde hier nur eine „kann“-Empfehlung ausgesprochen. Valide Therapieziele können auch nicht formuliert werden, da es sich hierbei stets um individuelles Vorgehen handelt. „Symptom-Kontrolle wäre ein theoretisches Ziel, da weder OS-Verlängerung noch Erhalt der Lebensqualität nachgewiesen sind. Das Behandlungsschema sollte sich jedoch nach der jeweiligen Vortherapie richten.

\subsection{Stellenwert der „Targeted Therapy“}

\begin{tabular}{|c|c|c|}
\hline 9.7. & Evidenzbasiertes Statement & $\begin{array}{l}\text { geprüft } \\
2018\end{array}$ \\
\hline $\begin{array}{l}\text { Level of } \\
\text { Evidence } \\
\text { 1b }\end{array}$ & \multicolumn{2}{|c|}{$\begin{array}{l}\text { Aufgrund eines nachgewiesenen Überlebensvorteils } \\
\text { besteht bei HER2-überexprimierenden Tumoren (IHC3 + } \\
\text { oder IHC2 + und FISH+) eine Indikation für den Einsatz von } \\
\text { Trastuzumab in Kombination mit Cisplatin und Fluoropy- } \\
\text { rimidinen (5-FU oder Capecitabin). }\end{array}$} \\
\hline & \multicolumn{2}{|c|}{ Leitlinienadaptation S3-LL Magenkarzinom [18] } \\
\hline
\end{tabular}

\begin{tabular}{|l|l|l|}
\hline 9.7. & Evidenzbasiertes Statement & $\begin{array}{l}\text { geprüft } \\
\mathbf{2 0 1 8}\end{array}$ \\
\hline $\begin{array}{l}\text { Konsens- } \\
\text { stärke }\end{array}$ & Starker Konsens $(100 \%)$ & \\
\hline
\end{tabular}

\section{Hintergrund}

Hierbei handelt es sich um eine Leitlinienadaptation für Patienten mit einem metastasierten oder lokal fortgeschrittenen, nicht kurativ behandelbaren Adenokarzinom des Ösophagus und des ösophagogastralen Übergangs.

In einer Phase-III-Studie (ToGA-Studie) verbesserte der HER2Antikörper Trastuzumab das OS und PFS von Patienten mit HER2positiven, fortgeschrittenen Magenkarzinomen und Adenokarzinomen des ösophagogastralen Überganges, deren Tumoren entweder immunhistochemisch HER2-positiv (IHC 3+) waren oder eine Amplifikation des HER2-Gens in der Fluoreszenz-insitu-Hybridisierung aufwiesen ( $\mathrm{FISH}+$ ). Zugelassen in Europa ist der Antikörper allerding nur, wenn die Tumoren IHC3 + oder $\mathrm{IHC2}+$ und FISH+. Interessanterweise scheinen die AEG I Tumoren, d. h. die distalen Adenokarzinome des Ösophagus (Barrettkarzinome) besonders häufig HER2 positiv zu sein [18, 228] (siehe auch Empfehlung 6.24.).

In einer Phase-3-Studie mit 780 Patienten wurde der Stellenwert von Pertuzumab in der Erstlinientherapie für Patienten mit HER2-positiven, fortgeschrittenen Magenkarzinomen und Adenokarzinomen des ösophagogastralen Überganges geprüft (JacobStudie, https://clinicaltrials.gov/ct2/show/NCT01 774 786). Der primäre Endpunkt einer signifikanten Überlebenszeitverlängerung wurde nicht erreicht. Das mediane Überleben lag für die Pertuzumab-basierte Kombination bei 17,5 Monaten gegenüber 14,2 Monaten für die Standardtherapie mit Trastuzumab, Cisplatin, Fluoropyrimidin (Capecitabin oder 5-Fluorouracil) (HR 0,84 $p=0,0565$ ). Damit ergibt sich derzeit keine Indikation für den zusätzlichen Einsatz von Pertuzumab in der Therapie des HER2positiven, fortgeschrittenen Magenkarzinoms und des Adenokarzinoms des ösophagogastralen Überganges (https://clinicaltrials. gov/ct2/show/NCT01774 786).

In einer Phase-3-Studie mit 345 Patienten wurde der Stellenwert von Trastuzumab-Emtansin (T-DM1) in der Zweitlinientherapie für Patienten mit HER2-positiven, fortgeschrittenen Magenkarzinomen und Adenokarzinomen des ösophagogastralen Überganges geprüft (Gatsby-Studie). Es zeigte sich für Trastuzumab-Emtansin kein Überlebensvorteil gegenüber einer Therapie mit einem Taxan (Docetaxel, Paclitaxel). Das mediane Überleben betrug 7,9 Monate für Trastuzumab-Emtansin und 8,6 Monate für die Taxan-behandelten Patienten (HR 1,15, $p=0,86$ ) [528].

Nach Progression unter Erstlinienchemotherapie wurde der VEGFR2-Antikörper Ramucirumab als Monotherapie (REGARD Studie) im Vergleich zu best supportive care [525] oder in Kombination mit Paclitaxel (RAINBOW Studie) [526] im Vergleich zu einer Paclitaxel Monotherapie eingesetzt. Das Gesamtüberleben wurde sowohl in der REGARD Studie (5,2 Monate vs. 3,8 Monate, HR 0,776) als auch in der Rainbow Studie (9,6 Monate vs. 7,4 Monate/HR 0,807) signifikant verbessert. Die Patienten schienen 
auch in Bezug auf die Lebensqualität zu profitieren. Beide Studien schlossen Patienten mit Magenkarzinom oder Adenokarzinomen des ösophagogastralen Übergangs ein. In der RAINBOW Studie zeigte sich auch eine höhere Ansprechrate in der Kombinationsgruppe.

\subsection{Palliative Radio(chemo)therapie}

\begin{tabular}{|l|l|l|}
\hline 9.8. & Konsensbasierte Empfehlung & $\begin{array}{l}\text { geprüft } \\
\mathbf{2 0 1 8}\end{array}$ \\
\hline EK & $\begin{array}{l}\text { Die perkutane Radiotherapie des Ösophaguskarzinoms - } \\
\text { ggfs. in Kombination mit einer simultanen Chemothera- } \\
\text { pie - kann bei lokalen Symptomen (z. B. Blutung, Stenose, } \\
\text { Kompression) im Rahmen der multidisziplinären Betreu- } \\
\text { ung eingesetzt werden. }\end{array}$ \\
\hline $\begin{array}{l}\text { Konsens- } \\
\text { stärke }\end{array}$ & Konsens (90\%) \\
\hline
\end{tabular}

\section{Hintergrund}

Das Ziel der palliativen Behandlung des Ösophaguskarzinoms ist die Linderung typischer Symptome der fortgeschrittenen Erkrankung wie Dysphagie, Schmerzen oder Blutungen. Eine Verlängerung des Überlebens ist durch eine palliative Therapie nicht zu erwarten [529]. Insofern ist für die Beurteilung palliativer Therapieverfahren die subjektive Beschwerdelinderung aus Sicht der Patienten von entscheidender Bedeutung.

Durch diese Betrachtung werden Therapieeffekt und Toxizität einer Therapie gleichermaßen erfasst.

Die verfügbare Literatur zur Beurteilung von Palliativtherapien anhand von Patientenbefragungen wurde von Amdal et al. in einer Übersichtsarbeit zusammengestellt [529].

Insgesamt 28 Studien, die Patientenbeurteilungen ausgewertet hatten, konnten identifiziert und ausgewertet werden. Der Vergleich der Ergebnisse nach Polychemotherapie, alleiniger Radiotherapie und einer Radiochemotherapie zeigte die Überlegenheit der Radiochemotherapie. Als Radiosensitizer wurden 5-FU oder Mitomycin C verwendet.

Während eine akzelerierte Radiochemotherapie mit hoher Akuttoxizität vergesellschaftet war, zeigte eine normofraktionierte Radiotherapie mit simultaner Gabe von 5-FU ein gutes Ansprechen bei geringer Toxizität [529, 530].

Die Entscheidung für eine lokale Palliativtherapie (perkutane Radiochemotherapie, Brachytherapie oder Stentimplantation) sollte in einem multidisziplinären Team abgestimmt werden [127].

Eine perkutane Radiotherapie 4 - 6 Wochen nach Stentimplantation verlängert das Dysphagie freie Intervall [531].

\subsection{Palliative Brachytherapie}

\begin{tabular}{|l|l|l|}
\hline $\mathbf{9 . 9 .}$ & Evidenzbasierte Empfehlung & $\begin{array}{l}\text { geprüft } \\
\mathbf{2 0 1 8}\end{array}$ \\
\hline $\begin{array}{l}\text { Empfeh- } \\
\text { lungsgrad } \\
\text { B }\end{array}$ & $\begin{array}{l}\text { Die palliative Brachytherapie sollte im Rahmen der multi- } \\
\text { disziplinären Betreuung von Patienten mit Ösophagus- } \\
\text { karzinom zur Linderung der Dysphagie gegebenenfalls } \\
\text { in Kombination mit einer Stentimplantation oder einer } \\
\text { perkutanen Radiochemotherapie angeboten werden }\end{array}$ \\
\hline $\begin{array}{l}\text { Level of } \\
\text { Evidence } \\
\mathbf{1 a}\end{array}$ & \begin{tabular}{l} 
Literatur: [530-533] \\
\hline $\begin{array}{l}\text { Konsens- } \\
\text { stärke }\end{array}$
\end{tabular} & Starker Konsens (100\%) \\
\hline
\end{tabular}

\section{Hintergrund}

Wie in zwei randomisierten Studien gezeigt wurde, kann die palliative Brachytherapie die Dysphagie und die Lebensqualität von Patienten mit inoperablen Ösophaguskarzinomen verbessern [529, 533]. Im Vergleich zur Stentimplantation tritt die Wirkung der Brachytherapie später ein, hält jedoch länger an. Eine Kombination von Stentimplantation mit der Brachytherapie ist möglich und sollte besonders bei Patienten zum Einsatz kommen, die eine längere projektierte Lebenserwartung haben. In dieser Situation kann die Brachytherapie die Dauer der Wirkung des Stents auf die Dysphagie verlängern [529, 532].

Bei einer ausgeprägten Dysphagie sollte zunächst eine Stentimplantation erfolgen, die $1-4$ Wochen später durch eine Brachytherapie ergänzt wird [531, 532].

Bei einer geringen Dysphagie erzeugt die alleinige Brachytherapie einen anhaltenden palliativen Effekt [532]und ist wegen der geringeren Komplikationsrate im Vergleich zur Stentimplantation als Therapie der Wahl anzusehen [533].

Die Wirkung der Brachytherapie kann durch eine zusätzliche perkutane Radiotherapie oder eine Radiochemotherapie verbessert werden [534].

Die Entscheidung für eine palliative Brachytherapie und deren Kombination mit einer Stentimplantation oder einer perkutanen Radiochemotherapie sollte in einem multidisziplinären Behandlungsteam abgestimmt werden.

\subsection{Endoskopische Stentapplikation}

\begin{tabular}{|l|l|}
\hline $\begin{array}{l}\text { 9.10. } \\
\text { Evidenzbasierte Empfehlung }\end{array}$ & $\begin{array}{l}\text { geprüt } \\
\mathbf{2 0 1 8}\end{array}$ \\
\hline $\begin{array}{l}\text { Empfeh- } \\
\text { lungsgrad } \\
\mathbf{B}\end{array}$ & $\begin{array}{l}\text { Zur raschen Linderung einer Dysphagie bei Patienten } \\
\text { mit Ösophaguskarzinom sollte ein selbstexpandierender } \\
\text { Metallstent eingesetzt werden. }\end{array}$ \\
\hline $\begin{array}{l}\text { Level of } \\
\text { Evidence } \\
\mathbf{1 a}\end{array}$ & Literatur: [535] \\
\hline $\begin{array}{l}\text { Konsens- } \\
\text { stärke }\end{array}$ & Konsens (90\%) \\
\hline
\end{tabular}




\section{Hintergrund}

Der Einsatz selbstexpandierender Metallstents (SEMS) zur schnellen Linderung der Dysphagie hat sich in den letzten Jahren als Standardtherapie etabliert $[53,535]$. Dies wurde auch durch eine Metaanalyse von 16 randomisierten Studien mit insgesamt 1027 Patienten belegt [535]. Nach Implantation eines SEMS können etwa 2/3 der Patienten mit stenosierendem Ösophaguskarzinom initial wieder feste Nahrung zu sich nehmen [536].

Allerdings war bei $10 \%$ der Patienten bereits 6 Wochen nach Stentimplantation die Dysphagie schlechter als initial [537]. Stents mit kleinem Durchmesser $(18 \mathrm{~mm})$ scheinen ähnlich effektiv zu sein wie breitere Stents $(24 \mathrm{~mm})$, aber induzieren weniger Schmerzen nach Implantation [537].

Es stehen eine Vielzahl von SEMS zur Verfügung. Nicht gecoverte SEMS sind initial gleich effektiv wie gecoverte Stents, jedoch besteht eine erhöhte Gefahr, dass Tumorgewebe durch die Maschen einwächst, sodass es bald wieder zu einer Tumorstenose kommen kann [538].

Ein Vorteil von ungecoverten SEMS ist allerdings eine niedrigere Migrationsrate. Der Einsatz von teilgecoverten SEMS scheint die Vorteile beider Typen zu vereinen, jedoch existieren hierzu kaum belastbare Daten. Bei Ösophaguskarzinomen mit Fisteln sind gecoverte Metallstents die Therapie der Wahl und haben eine Verschlussrate von 77 bis $92 \%$ [539, 540].

Selbstexpandierbare Plastikstents scheinen bezüglich der Linderung der Dysphagie ähnlich effektiv zu sein wie SEMS, aber die Migrationsrate ist deutlich erhöht [541]. Patienten mit einem Tumor im distalen Ösophagus leiden nach Stentimplantation häufig an massivem gastroösophagealen Reflux. Ob der Einsatz von SEMS mit Anti-Refluxventil eine Besserung des Reflux mit sich bringt, ist angesichts der Datenlage nicht sicher zu sagen. Drei prospektiv-randomisierte Studien liefern hier widersprüchliche Ergebnisse [542, 544].

Die Einlage eines SEMS bei gleichzeitiger Radiatio soll aufgrund der erhöhten Komplikationsrate vermieden werden. Auch die präoperative SEMS-Einlage als „Bridge-to-surgery“ oder vor einer geplanten perioperativen Radiochemotherapie wird nicht empfohlen, da sie mit einer erhöhten Inzidenz von Komplikationen einhergeht und mit der Einlage von Ernährungssonden valide Alternativen bestehen. In einer systematischen Meta-Analsyse von 9 Studien mit 180 Patienten zeigte sich, dass es zu einer hohen Rate an schweren Komplikationen kam (Stentmigration in 32 \% und Schmerzen in 51,4\%).[545]. Eine Europäische Kohortenstudie mit 2944 Patienten erbrachte ähnliche negative Ergebnisse [546]. Die präoperative Mortalität lag in der Stentgruppe bei $13,2 \%$ versus $8,6 \%$ in der Kontrollgruppe und die Morbidität bei $63,2 \%$ versus $59,2 \%$. Auch die R0-Resektionsrate, die mediane Zeit bis zum Rezidiv und das 3-Jahres-Überleben war in der SEMSGruppe deutlich schlechter als in der Gruppe ohne Stent.

Eine Alternative zur Palliation der Dysphagie bei Ösophaguskarzinomen ist die Brachytherapie. Im Vergleich zu Stents tritt hier die Linderung der Dysphagie verzögert ein, allerdings war die Brachytherapie der SEMS-Implantation nach 3 Monaten überlegen $[533,547]$.
Die Stentimplantation ist kosteneffektiver als die Brachytherapie [548]. Eine Kombination beider Methoden scheint effektiv und sicher zu sein, allerdings gibt es dafür kaum prospektive Daten [549].

\subsection{Stellenwert der intraluminalen lokalen Therapie}

\begin{tabular}{|l|l|l|}
\hline $\mathbf{9 . 1 1 .}$ & Konsensbasierte Empfehlung & $\begin{array}{l}\text { geprüft } \\
\mathbf{2 0 1 8}\end{array}$ \\
\hline EK & $\begin{array}{l}\text { Eine intraluminale thermoablative Therapie bei Patienten } \\
\text { mit exophytischem Ösophaguskarzinom in der palliativen } \\
\text { Situation kann erwogen werden. } \\
\text { Eine additive Brachytherapie oder Radiatio nach lokaler } \\
\text { Tumorablation kann das dysphagiefreie Intervall verlän- } \\
\text { gern. }\end{array}$ \\
\hline $\begin{array}{l}\text { Konsens- } \\
\text { stärke }\end{array}$ & \begin{tabular}{l} 
Starker Konsens (100\%) \\
\hline
\end{tabular} & \\
\hline
\end{tabular}

\section{Hintergrund}

Zur intraluminalen Tumortherapie stehen mehrere Methoden zur Verfügung. Eine palliative Therapie mittels photodynamischer Therapie (PDT) und intratumoraler Alkoholinjektion ist vor allem aufgrund der hohen Komplikationsrate und der Verfügbarkeit effektiverer Alternativmethoden obsolet [537, 550].

Für die Lasertherapie existieren die meisten Daten, welche die Effektivität belegen [550-555], allerdings steht heutzutage in den wenigsten Endoskopie-Abteilungen ein Laser zur Verfügung.

Die heute in Deutschland am häufigsten eingesetzte Methode zur thermoablativen Therapie ist die Argon-Plasma-Coagulation (APC) [556-558].

Sie scheint ähnlich effektiv zu sein wie die Lasertherapie, allerdings gibt es dafür kaum vergleichende Studien [556, 558]. Zur Tumorablation sind im Mittel 2,3 Sitzungen notwendig. Die Rate an schweren Komplikationen, wie Perforationen, lag in einer Serie unter $1 \%$ [556, 558].

Eine prospektive randomisierte Studie mit 93 Patienten verglich die alleinige APC-Therapie mit einer Kombinationstherapie von APC plus Hochdosis-Brachytherapie sowie APC plus PDT [559]. Die effektivste Therapie mit der geringsten Komplikationsrate war eine Kombination von APC mit Brachytherapie. Ein Rezidiv der Dysphagie trat erst nach 88 Tagen auf. Somit war das Dysphagie-freie Intervall signifikant länger als in den beiden Vergleichsgruppen (59 und 35 Tage).

Zur Frage, ob eine lokale Ablation von Metastasen bei Patienten mit Ösophaguskarzinom hinsichtlich Lebensqualität und Überleben gegenüber anderen therapeutischen Verfahren wie palliative Chemotherapie und BSC einen nachweisbaren Vorteil erbringt, gibt es aktuell keine Studien. In der Literatur finden sich lediglich Fallberichte oder Sammelkasuistiken. Dies gilt für Verfahren wie transarterielle Chemoembolisation (TACE), selektive interne Radiotherapie (SIRT), Radiofrequenzablation (RFA), Laser-induzierte Thermotherapie (LITT), Mikrowellenablation und stereotaktische Radiotherapie. Diese Verfahren sollten daher im Rahmen von kontrollierten, prospektiven Studien in dieser Indikation evaluiert werden. 


\section{Psychoonkologie}

\begin{tabular}{|l|l|l|}
\hline $\mathbf{1 0 . 1 .}$ & Konsensbasierte Empfehlung & $\begin{array}{l}\text { geprüft } \\
\mathbf{2 0 1 8}\end{array}$ \\
\hline EK & $\begin{array}{l}\text { Die psychoonkologische Versorgung von Patienten/innen } \\
\text { mit Ösophaguskarzinom sollte ein integraler Bestandteil } \\
\text { der onkologischen Diagnostik, Therapie und Nachsorge } \\
\text { sein und eine interdisziplinäre Aufgabe aller in der Onko- } \\
\text { logie beteiligten Berufsgruppen darstellen (vgl. S3-Leitli- } \\
\text { nie Psychoonkologie). }\end{array}$ \\
\hline $\begin{array}{l}\text { Konsens- } \\
\text { stärke }\end{array}$ & \begin{tabular}{l} 
Starker Konsens (100\%) \\
\hline
\end{tabular} & \\
\hline
\end{tabular}

\section{Hintergrund}

Patienten/innen mit einem Ösophaguskarzinom weisen eine Reihe von psychosozialen Belastungen auf [560]. Neben Beeinträchtigungen der psychischen Befindlichkeit, konnten Beeinträchtigungen der globalen Lebensqualität nachgewiesen werden, welche als Prädiktoren für eine erhöhte Mortalität angesehen werden können [561]. Insbesondere Beeinträchtigungen der sozialen Funktionsfähigkeit, Schmerz und ein vermindertes Aktivitätslevel postoperativ sind mit der Überlebenswahrscheinlichkeit verbunden [562]. Die soziale Funktionsfähigkeit und die Rollenfunktion zeigen auch bei Langzeitüberlebenden Beeinträchtigungen [563].

Für die Durchführung der psychoonkologischen Diagnostik, Beratung und Behandlung soll eine entsprechend qualifizierte Fachkraft zur Verfügung stehen. Die Feststellung des individuellen Bedarfs soll entsprechend der S3-Leitlinie Psychoonkologie (https:// www.leitlinienprogramm-onkologie.de/leitlinien/psychoonkologie/) über ein gestuftes Verfahren (standardisiertes Screeningverfahren plus ggf. diagnostisches Gespräch bei im Screening auffälligen Patienten) erfolgen [514, 564 - 566]. Der Einsatz eines psychoonkologischen Screeninginstrumentes sollte frühestmöglich erfolgen und in angemessenen Abständen, wenn klinisch indiziert oder bei Veränderung des Erkrankungsstatus der Patientin/des Patienten (z. B. bei Wiederauftreten oder Fortschreiten der Erkrankung) wiederholt durchgeführt werden. In der S3-Leitlinie Psychoonkologie wird vor allem die Verwendung des Distress Thermometers oder der Hospital Anxiety and Depression Scale (HADS) empfohlen [567 - 569]. Im Falle eines positiven Ergebnisses eines Screenings soll ein diagnostisches Gespräch zur weiteren diagnostischen Abklärung erfolgen.

Je nach Ausmaß der durch das Screening festgestellten psychosozialen Belastungen sollten gestuft psychosoziale Hilfen entsprechend der S3-Leitlinie Psychoonkologie in allen Krankheitsphasen den Patienten/innen und ihren Angehörigen angeboten werden. Dies beinhaltet, dass die/der Patient/in frühzeitig nach Diagnosestellung oder im Behandlungsverlauf über die Möglichkeiten psychoonkologischer Hilfestellungen informiert werden sollte. Hierbei sollte eine enge Absprache mit allen in die Versorgung des/r Patienten/in involvierten Personen (z. B. Hausarzt, ambulanter Pflegedienst) angestrebt und ein kontinuierlicher Informationsfluss sichergestellt werden.

Psychosoziale Hilfen beinhalten Maßnahmen zur Unterstützung der Krankheitsverarbeitung, des Umganges mit fortbestehenden Funktionsstörungen und weiteren krankheits- oder be- handlungsassoziierten Einschränkungen wie wirtschaftlichen Schwierigkeiten und Fragen zur Rückkehr ins Erwerbsleben. Somit betreffen diese Hilfen die gesamte Krankheitsphase von Diagnose, Aufklärung, Therapie, supportiver Behandlung, Rehabilitation, Nachsorge und ggf. palliativmedizinischer Versorgung.

Die Psychoonkologische Versorgung wird auf der Basis eines interdisziplinären Ansatzes zwischen allen an der Behandlung beteiligten Berufsgruppen realisiert [570]. Die psychoonkologischen Maßnahmen sollen in das Gesamtkonzept der onkologischen Behandlung integriert werden und durch regelhafte Rückmeldung mit den an der onkologischen Behandlung beteiligten Berufsgruppen optimiert werden. Hierfür sollte der behandelnde Arzt über psychoonkologische Basiskompetenzen verfügen. Darüber hinaus sollte eine psychoonkologische Fachkraft in das Behandlungsteam integriert sein, um die psychoonkologische Beratung und Behandlung durchführen zu können.

Die psychoonkologische Betreuung umfasst eine patientengerechte Information und Beratung, eine qualifizierte psychosoziale Diagnostik, sowie eine gezielte psychoonkologische Unterstützung bei der Bewältigung der Erkrankung, der Behandlung und der auftretenden Nebenwirkungen und Folgeprobleme.

Psychoonkologische Interventionen sind sowohl bei schweren psychischen Belastungen, Paarkonflikten sowie bei psychischen Störungen (insbesondere depressiven, Anpassungs- und Angststörungen) indiziert. Neben psychoedukativen und supportiven Interventionen wie Entspannungsverfahren etc. werden insbesondere die Einzelpsychotherapie sowie die Paartherapie eingesetzt [571]. Die psychoonkologischen Interventionen sollten am individuellen Bedarf des/r Patienten/in ausgerichtet werden. Ebenso sollte der Wunsch des/r Patienten/in berücksichtigt sowie der/die Partner/in und die Angehörigen in die psychosoziale Beratung und Behandlung des/r Patienten/in eingebunden werden.

\section{Palliativversorgung}

Palliativversorgung ist definiert als ein Ansatz zur Verbesserung der Lebensqualität von Patienten und ihren Familien, die mit Problemen konfrontiert sind, welche mit einer lebensbedrohlichen Erkrankung einhergehen. Dies geschieht durch Vorbeugen und Lindern von Leiden durch frühzeitige Erkennung, sorgfältige Einschätzung und Behandlung von Schmerzen sowie anderen Problemen körperlicher, psychosozialer und spiritueller Art. Die Palliativversorgung von Patienten mit einem Ösophaguskarzinom umfasst beispielsweise eine Reihe von Maßnahmen zur Linderung von Dysphagie, Übelkeit, Dyspnoe und/oder Schmerzen. An dieser Stelle sei auf die allgemeinen Empfehlungen hingewiesen, wie sie in der im Mai 2015 erschienenen „S3-Leitlinie Palliativmedizin für Patienten mit einer nicht heilbaren Krebserkrankung “ (AWMF-Registernummer: 128/001OL) ausführlich beschrieben werden (https://www.leitlinienprogramm-onkologie.de/leitlinien/palliativmedizin/).

Dort finden sich auch wichtige Empfehlungen zu Versorgungsstrukturen in der Palliativmedizin, inklusive eines Behandlungspfades für Patienten und Angehörige, da den Angehörigen bei der Betreuung dieser Patientengruppe eine wichtige Rolle zukommt. 
- Tab.13 Bewertungskriterien für potenzielle Qualitätsindikatoren.

Bitte bewerten Sie!

\section{Kriterium:}

Der Qualitätsindikator erfasst für den Patienten relevante Verbesserungspotenziale.

\section{Kriterium:}

Der Indikator ist klar und eindeutig definiert.

\section{Kriterium:}

Der Qualitätsindikator bezieht sich auf einen Versorgungsaspekt, der von den Leistungserbringer beeinflusst werden kann.

\section{Kriterium:}

Gibt es Risiken zur Fehlsteuerung durch den Indikator, die nicht korrigierbar sind?

\section{Kriterium:}

Die Daten werden beim Leistungserbringer routinemäßig dokumentiert oder eine zusätzliche Erhebung erfordert einen vertretbaren Aufwand

\section{Qualitätsindikatoren}

Qualitätsindikatoren (QI) sind Messgrößen, deren Erhebung der Beurteilung der Qualität der zugrunde liegenden Strukturen, Prozesse bzw. Ergebnisse dient [572, 573].

Qualitätsindikatoren sind ein wichtiges Instrument des Qualitätsmanagements zur Verbesserung der Versorgung. Dabei beleuchten Qualitätsindikatoren jeweils einen bestimmten Versorgungsaspekt. Die Erfüllung der in Qualitätsindikatoren beschriebenen Anforderungen kann deshalb nicht unbesehen mit der Qualität der Gesamtversorgung gleichgesetzt werden.

Die Methodik der Ableitung der vorliegenden Qualitätsindikatoren orientiert sich an der Methodik der Nationalen Versorgungsleitlinien [574]. Als Grundlage für die Qualitätsindikatoren dienten alle starken Empfehlungen (Empfehlungsgrad A), unabhängig von der Qualität der zugrundeliegenden Evidenz, d. h. inklusive Empfehlungen, die aufgrund eines Expertenkonsenses verabschiedet wurden. Die Empfehlungen wurden durch einen Methodiker in potenzielle ratenbasierte Indikatoren mit Nenner und Zähler überführt. Zudem wurden bestehende nationale und internationale Indikatoren recherchiert. Die potenziellen Indikatoren aus den Leitlinienempfehlungen und die bestehenden Indikatoren wurden von den Experten der AG Qualitätsindikatoren in einem zweistufigen Verfahren gesichtet und bewertet. In einem Präsenztreffen wurde eine erste Auswahl der potenziellen Qualitätsindikatoren im Hinblick auf ihre Relevanz für die Versorgung und ihre grundsätzliche Messbarkeit getroffen. Potenzielle Qualitätsindikatoren aus Empfehlungen mit inhaltlichen Doppelungen wurden nicht berücksichtigt. Es wurde auch geprüft, welche Anteile von Empfehlungen in bestehenden Tumordokumentationssystemen abbildbar sind (Basisdatensatz der Arbeitsgemeinschaft Deutscher Tumorzentren, zertifizierte onkologische/viszeralonkologische Zentren der DKG). Die als relevant und grundsätzlich messbar eingestuften potenziellen QI ( $n=15,2$ aus bestehenden QI) wurden im zweiten Schritt mit den in - Tab. 13 abgebildeten Kriterien schriftlich bewertet. Dazu wurden auch die Kodierungserfordernisse abgebildet. Zur korrekten Kodierung ist beim Ösophaguskarzinom neben der ICD eine Kodierung mithilfe der ICD-O (Internationale Klassifikation der Krankheiten für die Onkologie) erforderlich.

Als vorläufig angenommen galten Indikatoren mit mind. 75\% Zustimmung bei jedem schriftlich bewerteten Kriterium ( $n=9 / 15)$. Nach der schriftlichen Bewertung erfolgte eine methodisch moderierte Telefonkonferenz, in denen sowohl die angenommenen als auch die nicht angenommenen Indikatoren diskutiert wurden. Aufgrund der Klärung durch die Diskussion wurden ein schriftlich angenommener Indikator abgelehnt und zwei zunächst abgelehnte Indikatoren angenommen. Es wurde somit ein finales Set von elf Indikatoren verabschiedet, davon neun aus Empfehlungen der Leitlinie und 2 aus bestehenden Qualitätsindikatoren ( $\bullet$ Tab. 14).

Nicht als zu erhebender Qualitätsindikator (da als ratenbasierter Indikator nicht zielführend abbildbar), jedoch als künftige Zertifizierungs-Anforderung für ein viszeralonkologisches Zentrum der DKG wurde aufgenommen, dass Pathologen an Ringversuchen zur HER2-Bestimmung von Magen/ÖsophagusAdenokarzinomen teilnehmen sollen. Damit soll der 2. Teil der Empfehlung umgesetzt werden, die histopathologische Bestimmung des HER2-Status am Tumorgewebe qualitätsgesichert vorzunehmen.

Ebenso wurde das Einholen einer Zweitmeinung bei einem externen Pathologen (Referenzpathologie) bei Diagnose einer intraepithelialen Neoplasie im Barrett-Ösophagus als Zentrumsanforderung vorgeschlagen.

Die benannten Indikatoren sind als vorläufige Vorschläge zu verstehen. Eine ex ante Festlegung von Referenzbereichen (Bereichen, in denen eine unauffällige Qualität attestiert wird) ist nicht sicher möglich. Eine abschließende Bewertung kann erst nach einer Validierung der Indikatoren (u. a. im Hinblick auf Reliabilität und Validität) aufgrund erhobener Daten erfolgen. 
- Tab. 14 Vorschläge für Qualitätsindikatoren zur Versorgung von Patienten mit einem Plattenepithel- oder Adenokarzinom des Ösophagus.

$$
\text { Qualitätsindikator }
$$

$$
\text { Zugrundeliegende Empfehlung }
$$

\section{Evidenzgrundlage}

\section{QI 1: Vollständige histopathologische Befundung von Biopsiematerial}

(Vorschlag der Erfassung für 1 Jahr in DKG-zertifizierten viszeralonkologischen Zentren, danach Prüfung der weiteren Erfordernis) Anmerkung: „becherzellhaltige Barrettmukosa“ wird im ADT-Datensatz nicht erfasst.

\section{Zähler:}

Anzahl Patienten mit Angabe der Art der neo-plastischen Läsion (Low Grade Dysplasie/ Low Grade Intra Epitheliale Neoplasie, High Grade Dysplasie/High Grade Intraepitheliale Neoplasie $=\mathrm{C} 15 \mathrm{x}+8077 / 0,8077 / 2 ., \mathrm{C} 16 \mathrm{x}$, $+8148 / 0,8148 / 2$, Tis Klassifikation nach UICC, invasives Karzinom), WHO-hist. Typ, bei invasiven Karzinomen Grading nach aktueller WHOKlassifikation, Angabe, ob Biopsie aus dem distalen Ösophagus (C 15.5) mit becherzellhaltiger Barrettmukosa

Nenner:

Alle Patienten mit V. a. Neoplasie des

Ösophagus (D.00.1, C.15x., C16x) und Biopsie (1.440.9 und 1.440.a)

\subsection{9}

Der histopathologische Befund am Biopsiematerial soll die folgenden Angaben enthalten:

- Art der neoplastischen Läsion (LGD/LGIEN, HGD/HGIEN, Karzinom), insbesondere ob ein invasives Karzinom vorliegt (bei HGD/HGIEN: Klassifikation am Biopsat als Tis nach UICC)

- Histologischer Typ nach WHO (insbesondere Unterscheidung Plattenepithel- versus Adenokarzinom)

- Bei invasiven Adenokarzinomen: Differenzierungsgrad (Grading) nach aktueller WHO-Klassifikation

- Bei Läsionen im distalen Ösophagus: ist eine Becherzellhaltige Barrett-Mukosa vorhanden?
Expertenkonsens Konsensstärke:

$100 \%$

Im begründenden Hintergrundtext keine Literaturzitate angegeben.

QI 2: Vollständige histopathologische Befundung von lokalen Exzidaten

(Für die Erhebung dieses Indikators sollen Datenfelder für die Angabe des zirkulären und basalen Resektionsrandes und der Invasionstiefe in das spezifische Modul des allgemeinen Basisdatensatzes der ADT aufgenommen werden. Die Größe in drei Dimensionen und die zusammenfassende Einschätzung des LK-Metastasierungsrisikos sind nicht dokumentierbar.)

\section{Zähler:}

Anzahl Patienten mit Angabe der Art der neoplastischen Läsion $(C 15 x+8077 / 0,8077 / 2$., C16x, +8148/0, 8148/2), WHO-Klass., Grading, Lymphgefäß- und/oder Veneninvasion, Tiefe (Invasionstiefe) + Angabe von zirkulärem und basaler Resektionsrand

Nenner:

Alle Patienten mit Neoplasie des Ösophagus (D.00.1, C.15x, C16x) und endoskopischer Resektion (5.422.2, 5.422.0, 5.422.2,5.422.3)
6.21.

Der histopathologische Befund an lokalen Exzidaten (endoskopische Resektion; ER) soll folgende Angaben enthalten:

- Größe der neoplastischen Läsion in 3 Dimensionen, wenn möglich

- Art der neoplastischen Läsion (LGD/LGIEN, HGD/HGIEN, Karzinom) - insbesondere, ob ein invasives Karzinom vorliegt (bei HGD/HGIEN: Klassifikation am Resektat als pTis nach UICC)

- Bei Karzinomnachweis: Histologischer Typ nach WHO (insbesondere Unterscheidung Plattenepithel- versus Adenokarzinom, andere seltene Typen)

- Bei invasiven Adenokarzinomen: Differenzierungsgrad (Grading) nach aktueller WHO-Klassifikation

- Maximale Tiefe der Infiltration: pT1a (m1, m2, m3, m4)/ pT1b (sm1, sm2, sm3) plus Infiltrationstiefe in $\mu \mathrm{m}$ (oder höhere $\mathrm{pT}$-Kategorie)

- Lymphgefäß- und/oder Veneninvasion (L0 vs. L1, V0 vs. V1)

- Zusammenfassende Einschätzung des LK-Metastasierungsrisikos: Low risk vs. High risk-Resektionsränder bzgl. der Neoplasie (bei ER in toto zirkulärer und basaler RR; bei „piecemeal“-ER basaler RR, da hier der zirkuläre RR in der Regel histo-pathologisch als RX gewertet werden muss)
Expertenkonsens Konsensstärke: $100 \%$ Literatur: [200-202]

\section{QI 3: Vollständige histopathologische Befundung des Operationsresektates}

Anmerkung: Die Lokalisation des Tumorzentrums in Beziehung zur ÖGJ (Ösophagogastraler Junktion) und Angabe, ob der Tumor die ÖG] kreuzt ist nicht dokumentierbar.

Zähler:
Anzahl Patienten mit Angabe von Größe der neoplastischen Läsion, Art der Läsion (C15x $+8077 / 0,8077 / 2 .$, C16x, +8148/0, 8148/2, Tis), WHO-Klass. Grading, PT, pN, Ratio LK, L, V, R-Status (TNM)
6.22 .

Der histopathologische Befund an Operationsresektaten soll folgende Angaben enthalten:

- Größe der neoplastischen Läsion Lokalisation des Tumorzentrums in Beziehung zur ÖG] und Angabe, ob der Tumor die ÖG] kreuzt
Expertenkonsens Konsensstärke: $100 \%$

Literatur:

[200 - 202, 204, $206-209,211$ 227] 
- Tab. 14 (Fortsetzung)

\section{Qualitätsindikator}

\section{Nenner:}

Alle Patienten mit Neoplasie des Ösophagus und chirurgischer Resektion (D.00.1, C.15x, C16x) und chirurgischer Resektion (OPS

5.422 .0 , alle $5.423,5.424,5.425,5.426)$

\section{Zugrundeliegende Empfehlung}

- Art der neoplastischen Läsion (LGD/LGIEN, HGD/HGIEN, Karzinom) - insbesondere, ob ein invasives Karzinom vorliegt (bei HGD/HGIEN: Klassifikation als pTis nach UICC)

- Bei Karzinomnachweis: Histologischer Typ nach WHO (insbesondere Unterscheidung Plattenepithel- versus A-denokarzinom, andere seltene Typen) Differenzierungsgrad (Grading)

- Maximale Tiefe der Infiltration (pT)

- Lymph- oder Hämangio-Invasion: L0 vs. L1, V0 vs. V1)

- Resektionsränder (oral, aboral und zirkumferenziell): R0 / R1

- Lymphknoten-Status nach UICC (pN) und Ratio aus Zahl der befallenen und untersuchten Lymphknoten (.../...LK)

\section{Evidenzgrundlage}

QI 4: Therapieempfehlung aus interdisziplinärer Tumorkonferenz

(Anmerkung: Es wurde nur der erste Teil der Empfehlung für operationalisierbar eingeschätzt. Die Leitlinienautoren sprachen sich für eine Festlegung der Teilnehmer der Tumorkonferenz durch die Zertifizierungskommission der DKG für viszeralonkologische Zentren aus. Erfasst werden soll der Primärfall)

\section{Zähler: 8.1 .}

Anzahl Patienten mit Therapieempfehlung aus interdisziplinärer Tumorkonferenz vor Therapie (Staging abgeschlossen)

Nenner:

Alle Patienten mit Neoplasie des Ösophagus (D.00.1, C.15x, C16x)
1. Therapieempfehlungen sollen in einer interdisziplinären Tumorkonferenz getroffen werden.

2 Als Grundlage für die Therapieempfehlung sollen StagingInformationen, die Patienten-Komorbiditäten, der Ernährungsstatus und die Patientenpräferenz berücksichtigt werden.
Expertenkonsens Konsensstärke $100 \%$

kein Literaturzitat angegeben

QI 5: Vollständige endoskopische Resektion einer intraepithelialen Neoplasie oder eines mukosalen Frühkarzinoms im Barrett-Ösophagus

(Anmerkung: nur die Teile a+b der Empfehlung wurden umgesetzt. „keine Ulzerationen“ nicht in Dokumentationssystemen abgebildet)

\section{Zähler:}

Anzahl Patienten mit R0

Nenner:

Alle Patienten mit Diagnose einer hochgradigen intraepithelialen Neoplasie (C16x, 8148/2) oder eines mukosalen Karzinoms ( $=8140 / 3)$ L0, V0, G1 / G2, keine Ulzerationen, Infiltrationstiefe $\leq$ m3 im Barrett-Ösophagus (K22.7) und endoskopischer Resektion (5.422.2, 5.422.20., $5.422 .3,5.422 .4)$
8.2.

a. Bei Nachweis einer hochgradigen intraepithelialen Neoplasie oder eines mukosalen Karzinoms (LO, V0, keine Ulzerationen, Grading G1/G2, Infiltrationstiefe $\leq \mathrm{m} 3$ ) im Barrett-Ösophagus soll eine endoskopische Resektion durchgeführt werden, da hierdurch neben der Therapie auch ein Staging der Läsion mit der Frage der Tiefeninfiltration erfolgt.

Daher ist eine endoskopisch komplette Resektion mit kurativer Intention anzustreben.

Nach erfolgreicher Resektion von Neoplasien im Barrett-Ösophagus soll die nicht neoplastische Barrett-Mucosa thermisch abladiert werden, um die Rate an metachronen Neoplasien zu senken.
Expertenkonsens Konsensstärke $100 \%$

Literatur: [111, $237-251,253-$

255]

\section{QI 6: Vollständige chirurgische Resektion}

\section{Zähler:}

Anzahl Patienten mit R0

\section{Nenner:}

Alle Patienten mit Neoplasie des Ösophagus

(D.00.1, C.15x, C16x) und Operation (chirurgi-

sche Resektion OPS 5.422.0, alle 5.423, 5.424,

$5.425,5.426$ )
Q1 7: präoperative Radiotherapie bei Patienten mit einem Plattenepithelkarzinom des Ösophagus T3/T4 Anmerkung: Nur der erste Teil der Empfehlung wurde umgesetzt

\section{Zähler:}

Anzahl Patienten mit präoperativer

Radiochemotherapie

Nenner:

Alle Patienten mit Plattenepithelkarzinom des Ösophagus (C15x) und cT3 / cT4
Bei operablen Patienten mit einem Plattenepithelkarzinom de Ösophagus der Kategorie cT3 und bei resektablen cT4 Tumoren soll eine präoperative Radiochemotherapie mit anschlieBender kompletter Resektion durchgeführt werden.

\section{9.}

Das Ziel der chirurgischen Resektion beim Plattenepithelkarzinom und Adenokarzinom ist die vollständige Entfernung des Tumors (oral, aboral und in der Zirkumferenz) und der regionären Lymphknoten.
Expertenkonsens Konsensstärke $100 \%$
Evidenzgrad 1a, Konsensstärke $100 \%$

Literatur: [406-409] 
- Tab. 14 (Fortsetzung)

Qualitätsindikator

Zugrundeliegende Empfehlung

Evidenzgrundlage

QI 8: perioperative Chemotherapie oder präoperative Radiochemotherapie bei operablen Patienten mit Adenokarzinom des Ösophagus

\section{Zähler:}

Anzahl Patienten mit prä- und postoperativer

Chemotherapie oder präoperativer Radioche-

motherapie

Nenner:

Alle Patienten mit Adenokarzinom des Ösophagus (C.16x, 8140/3) und Operation (OPS 5.422 .0 , alle $5.423,5.424,5.425,5.426)$ und cT3 oder CT4

\subsection{4 .}

Beim operablen Patienten mit Adenokarzinom des Ösophagus oder des ösophagogastralen Übergangs der Kategorie CT3 und bei resektablen cT4 Tumoren soll eine perioperative Chemotherapie oder eine präoperative Radiochemotherapie durchgeführt werden.
Evidenzgrad 1a Konsensstärke

$100 \%$

Literatur:

[389, $391-393,402,403]$

\section{QI 9: Systemtherapie des metastasierten Ösophaguskarzinoms}

\section{Zähler:}

Anzahl Patienten mit systemischer

Chemotherapie (Erstlinie)

\section{Nenner:}

Alle Patienten mit einem metastasierten

Adenokarzinom des Ösophagus

(C16.x, 8140/3, M1)
9.1.

Patienten mit einem metastasierten oder lokal fortgeschrittenen, nicht kurativ behandelbaren Adenokarzinom des Ösophagus soll eine systemische Chemotherapie angeboten werden. Therapieziel ist die Verlängerung der Überlebenszeit und der Erhalt der Lebensqualität.
Evidenzgrad 1a Literatur:

$[111,498,502]$

\section{QI 10: Anastomoseninsuffizienz nach chirurgischer Resektion}

\section{Zähler:}

Anzahl Patienten mit Anastomoseninsuffizienz (ICD: K91.83 „Insuffizienzen von Anastomosen und Nähten nach OP an: Anus, Darm, Magen, Ösophagus, Rektum), die endoskopisch, interventionell oder operativ behandelt wurden Nenner:

Alle Patienten mit Neoplasie des Ösophagus (D.00.1, C.15x, C16x) und Operation (chirurgische Resektion OPS 5.422.0, alle 5.423, 5.424, $5.425,5.426)$

\section{QI 11.1 und 11.2: Mortalität nach Operation}

Zähler 11.1:

Anzahl postoperativ verstorbene Patienten nach 30 Tagen

\section{Zähler $\mathbf{1 1 . 2}$}

Anzahl postoperativ verstorbene Patienten nach 90 Tagen

Nenner 11. 1 +11.2:

Alle Patienten mit Neoplasie des Ösophagus (D.00.1, C.15x, C16x) und Operation (chirurgische Resektion OPS 5.422.0, alle 5.423, 5.424, $5.425,5.426)$
Ergebnisindikator basierend auf einem entsprechenden QI aus Belgien:

„OC9: Proportion of patients experiencing anastomotic leakage after oesophagectomy"

Vorschlag Prof. Hölscher:

Einteilung der Anastomoseninsuffizienz in I-III.

I = locally defect, no change in therapy, only medicaments or diet modification

II: Localized defect requiring intervention, but no surgery, e. g.

IR drain, stent or bedside opening

III: Localized defect requiring surgical therapy - Soll erfasst werden

Ergebnisindikator basierend auf einem entsprechenden QI aus Belgien:

OC6: Oesophageal resection mortality rate within 30 days [575]
Definition as in Low et al, International Consensus on Standardization of Data Collection for Complications Associated With Esophagectomy: Esophagectomy Complications Consensus Group (ECCG)., 2015 [348]

Geht als Vorschlag auf folgende Publikationen zurück:

[576, 577]:

Mortality Rate: 90 days better than 30 days to measure. 


\section{Anhänge}

\section{1. Übersicht der Änderungen in Version 2.0 (2018)}

- Tab.15 geänderte Empfehlung und Statement in Version 2.0.

\section{Version 1.0 (2015)}

Level of Evidence: 3a

Alkohol erhöht das Risiko für Plattenepithelkarzinome des Ösophagu

Level of Evidence: 3a

Gastroösophagealer Reflux erhöht das Risiko für Adenokarzinome des Ösophagus.

Die Chromoendoskopie des ganzen Ösophagus mit Lugol'scher Lösung scheint für „Hoch-Risiko-Patienten “ (= Plattenepithelkarzinom des Mundes/Nase/Rachens/Bronchial-Systems; ggf. erhöhter Nikotin- und Alkoholkonsum) sinnvoll zur Detektion früher Plattenepithel-Neoplasien zu sein und kann in dieser Situation eingesetzt werden.

Die Chromoendoskopie sowie die computergestützte Chromoendoskopie können zur Verbesserung der Detektionsrate von Dysplasien/Frühkarzinomen eingesetzt werden.

Level of Evidence: $2 a$

Der endoskopische Ultraschall (EUS) sollte Bestandteil des Stagings bei Patienten mit kurativer Therapieintention sein.

Level of Evidence: 4

Bei lokal fortgeschrittenen Tumoren (cT 2-4 und $\mathrm{cN}+$ ) kann zusätzlich eine PET/CT-Untersuchung zum M-Staging erwogen werden, falls der Patient potenziell kurativ behandelbar ist bzw. das Ergebnis klinische Konsequenzen hat

EK

Eine diagnostische Laparoskopie kann bei Adenokarzinomen des distalen Ösophagus und des ösophagogastralen Überganges zum Ausschluss von Metastasen der Leber und/oder des

Peritoneums in fortgeschrittenen Stadien durchgeführt werden (insbesondere im Falle einer cT3-, cT4-Kategorie).

EK

Bei Diagnose einer intraepithelialen Neoplasie (Dysplasie) im Barrett-Ösophagus soll eine Referenzpathologie durch einen externen Pathologen eingeholt werden.

EK

Der histopathologische Befund an Resektaten sollte nach präoperativer Therapie (neoadjuvanter Therapie) zusätzlich Aussagen zum Regressions-Score enthalten.

EK

Bei Lokalisation des Tumors

- Im ösophagogastralen Übergang (AEG Typ II) mit ausgedehnter Infiltration der unteren Speiseröhre kann eine transthorakale subtotale Ösophagektomie durchgeführt werden, alternativ kann eine transhiatale abdomino-cervicale subtotale Ösophagektomie erfolgen. Bei zusätzlich ausgedehntem Magenbefall kann eine Ösophago-Gastrektomie erforderlich sein.

- im distalen (incl. AEG Typ I) und mittleren thorakalen Ösophagus sollte eine transthorakale subtotale Ösophagektomie durchgeführt werden.

- im oberen thorakalen Ösophagus sollte das Resektionsausmaß zur Wahrung des Sicherheitsabstandes nach oral ausgedehnt werden.

\section{Version $2.0(2018)$}

Level of Evidence: $2 b$

Alkohol erhöht das Risiko für Plattenepithelkarzinome des Ösophagu

Level of Evidence: $2 b$

Gastroösophagealer Reflux erhöht das Risiko für Adenokarzinome des Ösophagus.

Die Chromoendoskopie (Lugol'sche Lösung) oder die computergestützten digitalen (Filter-) Verfahren sollten bei Risiko-Patienten für ein Ösophaguskarzinom (= anamnestisch Plattenepithelkarzinom des Mundes/Nase/Rachens/Bronchial-Systems, Ösophagus) eingesetzt werden.

Die Chromoendoskopie oder die computergestützten digitalen (Filter-) Verfahren sollten zur Verbesserung der Detektion von Dysplasien/Frühkarzinomen eingesetzt werden.

Level of Evidence: $1 \mathrm{~b}$

Der endoskopische Ultraschall (EUS) sollte Bestandteil des Stagings bei Patienten mit kurativer Therapieintention sein.

Level of Evidence: $1 \mathrm{~b}$

Bei lokal fortgeschrittenen Tumoren (cT 2-4 und cN+) kann zusätzlich eine PET/CT-Untersuchung zum M-Staging eingesetzt werden, falls der Patient potenziell kurativ behandelbar ist bzw. das Ergebnis klinische Konsequenzen hat.

Level of Evidence: $1 \mathrm{~b}$

Eine diagnostische Laparoskopie kann bei Adenokarzinomen des ösophagogastralen Überganges zum Ausschluss von Metastasen der Leber und/oder des Peritoneums in fortgeschrittenen Stadien durchgeführt werden (insbesondere im Falle einer cT3-, cT4-Kategorie).

EK

Bei histologischer Diagnose einer IEN/Dysplasie im Barrett-Ösophagus soll der Prozess einer kompetenten (dokumentierten) pathologischen Zweitmeinung im Sinne eines Vier-Augen-Prinzips durchgeführt werden. Bei Dissens oder Unsicherheit bezüglich der Dysplasiediagnose soll eine externe Begutachtung erfolgen.

Level of Evidence: $2 \mathrm{~b}$

Der histopathologische Befund an Resektaten sollte nach präoperativer Therapie (neoadjuvanter Therapie) zusätzlich Aussagen zum Regressions-Score enthalten.

EK

Bei Lokalisation des Tumors

- im ösophagogastralen Übergang (AEG Typ III) sollte eine totale Gastrektomie mit distaler Ösophagusresektion ausgeführt werden.

- im ösophagogastralen Übergang (AEG Typ II) kann eine totale Gastrektomie mit distaler Ösophagusresektion, eine transthorakale subtotale Ösophagektomie und alternativ eine transhiatale abdominocervicale subtotale Ösophagektomie erfolgen. Eine ausgedehnte Infiltration der unteren Speiseröhre favorisiert dabei mehr die Ösophagektomie, eine ausgedehnte Infiltration des subcardialen Magens mehr die Gastrektomie. Bei langstreckigem Befall beider Organe kann eine totale Ösophagogastrektomie erforderlich sein. 
- Tab. 15 (Fortsetzung)

\section{Version 1.0 (2015)}

- Im zervikalen Ösophagus soll die Indikation zum chirurgischen Vorgehen im Vergleich zur definitiven Radiochemotherapie unter eingehender Nutzen/ Risikoabwägung diskutiert werden. Als chirurgisches Verfahren kann entweder

- eine totale Ösophagektomie oder in geeigneten Fällen eine zervikale Ösophagusresektion über einen zervikalen Zugang mit oberer Sternotomie erfolgen.
Version 2.0 (2018)

- im distalen (incl. AEG Typ I) und mittleren thorakalen Ösophagus sollte eine transthorakale subtotale Ösophagektomie durchgeführt werden.

- im oberen thorakalen Ösophagus sollte das Resektionsausmaß zur Wahrung des Sicherheitsabstandes nach oral ausgedehnt werden.

- im zervikalen Ösophagus soll die Indikation zum chirurgischen Vorgehen im Vergleich zur definitiven Radiochemotherapie unter eingehender Nutzen/Risikoabwägung diskutiert werden (siehe hierzu auch Empfehlung 8.32.). Als chirurgisches Verfahren kann entweder Ösophagusresektion über einen zervikalen Zugang mit oberer Sternotomie erfolgen. eine totale Ösophagektomie oder in geeigneten Fällen eine zervikale

\section{Abbildungsverzeichnis}

\begin{tabular}{l|l|l|}
$\begin{array}{l}\text { Abb. 1: Bekannte Risikofaktoren für die Entstehung eines } \\
\text { Ösophaguskarzinoms }\end{array}$ & 346 \\
\hline $\begin{array}{l}\text { Abb. 2: Algorithmus zum Staging des Ösophaguskarzi- } \\
\text { noms }\end{array}$ & 356 \\
\hline $\begin{array}{l}\text { Abb. 3: Therapiealgorithmus bei funktionell operablen und } \\
\text { onkologisch resektablen Adenokarzinomen des Ösophagus } \\
\text { und des gastroösophagealen Übergangs }\end{array}$ & 379 \\
\hline $\begin{array}{l}\text { Abb. 4: Therapiealgorithmus bei funktionell operablen und } \\
\text { onkologisch resektablen Plattenepithelkarzinomen des } \\
\text { Ösophagus. Zur Therapie mittels definitiver Radiochemo- } \\
\text { therapie bei lokalisierten Plattenepithelkarzinomen des } \\
\text { zervikalen Ösophagus siehe Empfehlung } 8.32 .\end{array}$ & 380 \\
\hline
\end{tabular}

\section{Tabellenverzeichnis}

- Tab. 1: Beteiligte Fachgesellschaften und Organisationen

- Tab. 2: Steuergruppe und Arbeitsgruppen und deren Mitglieder

- Tab. 3: Schema der Evidenzgraduierung nach Oxford (Version März 2009)

- Tab. 4: Schema der Empfehlungsgraduierung

- Tab. 5: Konsensusstärke

- Tab. 6: Übersicht über die Staging-Ergebnisse des endoskopischen Ultraschalls (EUS) für die T- und N-Kategorien von Ösophaguskarzinomen (Sensitivität/Spezifität durch EUS/EUS-FNP)

- Tab. 7: Direkter Vergleich der eingesetzten Stagingverfah356 ren bei der Aufdeckung von Lymphknoten-Metastasen [92-95].

- Tab. 8: Klinische Klassifikation der Ösophaguskarzinome, eingeschlossen Karzinome des ösophagogastralen Übergangs nach der TNM-Klassifikation [196]

- Tab. 9: Stadiengruppierung der TNM-Klassifikation der phagogastralen Übergangs [196] - Plattenepithelkarzinome

- Tab. 10: Stadiengruppierung der TNM-Klassifikation der Ösophaguskarzinome, eingeschlossen Karzinome des ösophagogastralen Übergangs [196] - Adenokarzinome Ösophaguskarzinome, eingeschlossen Karzinome des öso-
- Tab. 11: Tumorregressions-Score für Adenokarzinome nach Becker et al. [216 - 219]

- Tab. 12: Mögliche Chemotherapieregime bei neoadju366 vanter präoperativer Radiochemotherapie [413 - 415]

- Tab. 13: Bewertungskriterien für potenzielle Qualitätsindikatoren

- Tab. 14: Vorschläge für Qualitätsindikatoren zur Versorgung von Patienten mit einem Plattenepithel- oder Adenokarzinom des Ösophagus

- Tab. 15: geänderte Empfehlung und Statement in Version 2.0
[3] Bayley MT et al. Where to build the bridge between evidence and practice?: results of an international workshop to prioritize knowledge translation activities in traumatic brain injury care. J Head Trauma Rehabil 2014; 29: $268-276$

[4] Dear RF et al. Identifying and prioritising gaps in colorectal cancer trials research in Australia. Med J Aust 2012; 197: $507-511$

[5] Miake-Lye IM et al. What is an evidence map? A systematic review of published evidence maps and their definitions, methods, and products. Syst Rev 2016; 5: 28

[6] Clavisi $O$ et al. Effective stakeholder participation in setting research priorities using a Global Evidence Mapping approach. J Clin Epidemiol 2013; 66: 496-502.e2 
[7] Tramacere I, La VC, Negri E. Tobacco smoking and esophageal and gastric cardia adenocarcinoma: a meta-analysis. Epidemiology 2011; 22: $344-349$

[8] Lee $\mathrm{CH}$ et al. Anatomical subsite discrepancy in relation to the impact of the consumption of alcohol, tobacco and betel quid on esophageal cancer. Int J Cancer 2007; 120: 1755 - 1762

[9] Pandey $\mathrm{N}$ et al. Gastro-oesophageal reflux symptoms and the risks of oesophageal cancer: are the effects modified by smoking, NSAIDs or acid suppressants? Gut 2010; 59: 31 - 38

[10] Rafiq R et al. Secondhand Smoking and the Risk of Esophageal Squamous Cell Carcinoma in a High Incidence Region, Kashmir, India: A Case-control-observational Study. Medicine (Baltimore) 2016; 95 : e2340

[11] Sewram V et al. Tobacco and alcohol as risk factors for oesophageal cancer in a high incidence area in South Africa. Cancer Epidemiol 2016; 41: $113-121$

[12] Islami F et al. Alcohol drinking and esophageal squamous cell carcinoma with focus on lightdrinkers and never-smokers: a systematic review and meta-analysis. Int J Cancer 2011; 129: 2473-2484

[13] Bagnardi $V$ et al. Light alcohol drinking and cancer: a meta-analysis. AnnOncol 2013; 24: $301-308$

[14] Freedman ND et al. Alcohol intake and risk of oesophageal adenocarcinoma: a pooled analysis from the BEACON Consortium. Gut 2011; 60: 1029- 1037

[15] Pandeya $\mathrm{N}$ et al. Alcohol consumption and the risks of adenocarcinoma and squamous cell carcinoma of the esophagus. Gastroenterology 2009; 136: $1215-1224$, e1-2

[16] Tramacere I et al. A meta-analysis on alcohol drinking and esophageal and gastric cardia adenocarcinoma risk. Ann Oncol 2012; 23: 287-297

[17] Ji J, Sundquist J, Sundquist K. Associations of alcohol use disorders with esophageal and gastric cancers: a population-based study in Sweden. Eur J Cancer Prev 2017; 26: 119-124

[18] Moehler M et al. S3-Leitlinie „Magenkarzinom“. Z Gastroenterol 2011; 49: $461-531$

[19] Guh DP et al. The incidence of co-morbidities related to obesity and overweight: a systematic review and meta-analysis. BMC Public Health 2009; 9: 88

[20] Ryan AM et al. Obesity, metabolic syndrome and esophageal adenocarcinoma: epidemiology, etiology and new targets. Cancer Epidemiol 2011; 35: 309-319

[21] Turati F et al. A meta-analysis of body mass index and esophageal and gastric cardia adenocarcinoma. Ann Oncol 2013; 24: 609-617

[22] O'Doherty MG et al. A prospective cohort study of obesity and risk of oesophageal and gastric adenocarcinoma in the NIH-AARP Diet and Health Study. Gut 2012; 61: 1261 - 1268

[23] Thota PN et al. Influence of body mass index on the prevalence and progression of dysplasia in Barrett's esophagus: a retrospective analysis (.). Scand J Gastroenterol 2016; 51: 1288-1293

[24] Krishnamoorthi R et al. Rates and predictors of progression to esophageal carcinoma in a large population-based Barrett's esophagus cohort. Gastrointest Endosc 2016; 84: 40 -46.e7

[25] Leeuwenburgh I et al. Long-term esophageal cancer risk in patients with primary achalasia: a prospective study. Am J Gastroenterol 2010; 105: 2144- 2149

[26] Zendehdel $\mathrm{K}$ et al. Risk of esophageal adenocarcinoma in achalasia patients, a retrospective cohort study in Sweden. Am J Gastroenterol 2011; 106: $57-61$

[27] Ji ], Hemminki K. Familial risk for esophageal cancer: an updated epidemiologic study from Sweden. Clin Gastroenterol Hepatol 2006; 4: 840 845

[28] Morton LM et al. Risk of treatment-related esophageal cancer among breast cancer survivors. Ann Oncol 2012; 23: 3081 - 3091
[29] Zablotska LB et al. Increased risk of squamous cell esophageal cancer after adjuvant radiation therapy for primary breast cancer. Am J Epidemiol 2005; 161: $330-337$

[30] Levi F et al. Increased risk of esophageal cancer after breast cancer. Ann Oncol 2005; 16: 1829-1831

[31] Roychoudhuri R et al. Radiation-induced malignancies following radiotherapy for breast cancer. Br J Cancer 2004; 91: 868-872

[32] Okonta KE, Tettey M, Abubakar U. In patients with corrosive oesophageal stricture for surgery, is oesophagectomy rather than bypass necessary to reduce the risk of oesophageal malignancy? Interact Cardiovasc Thorac Surg 2012; 15: 713-715

[33] Lagergren J et al. Symptomatic gastroesophageal reflux as a risk factor for esophageal adenocarcinoma. N Engl J Med 1999; 340: 825-831

[34] Velanovich $V$ et al. Relationship of gastroesophageal reflux disease with adenocarcinoma of the distal esophagus and cardia. Dig Surg 2002; 19 : $349-353$

[35] Wu AH, Tseng CC, Bernstein L. Hiatal hernia, reflux symptoms, body size, and risk of esophageal and gastric adenocarcinoma. Cancer 2003; 98: $940-948$

[36] Chak A et al. Gastroesophageal reflux symptoms in patients with adenocarcinoma of the esophagus or cardia. Cancer 2006; 107: 2160-2166

[37] Rubenstein JH, Taylor JB. Meta-analysis: the association of oesophageal adenocarcinoma with symptoms of gastro-oesophageal reflux. Aliment Pharmacol Ther 2010; 32: 1222-1227

[38] Hazelton WD et al. The Role of Gastroesophageal Reflux and Other Factors during Progression to Esophageal Adenocarcinoma. Cancer Epidemiol Biomarkers Prev 2015; 24: 1012 - 1023

[39] Hvid-Jensen F et al. Incidence of adenocarcinoma among patients with Barrett's esophagus. N Engl J Med 2011; 365: 1375-1383

[40] Wani S et al. Patients with nondysplastic Barrett's esophagus have low risks for developing dysplasia or esophageal adenocarcinoma. Clin Gastroenterol Hepatol 2011; 9: 220 - 227; quiz e26

[41] Anaparthy $R$ et al. Association between length of Barrett's esophagus and risk of high-grade dysplasia or adenocarcinoma in patients without dysplasia. Clin Gastroenterol Hepatol 2013; 11: 1430-1436

[42] Gaddam S et al. Persistence of nondysplastic Barrett's esophagus identifies patients at lower risk for esophageal adenocarcinoma: results from a large multicenter cohort. Gastroenterology 2013; 145: 548 - 553.e1

[43] Desai TK et al. The incidence of oesophageal adenocarcinoma in nondysplastic Barrett's oesophagus: a meta-analysis. Gut 2012; 61: 970 976

[44] Solaymani-Dodaran M, Card TR, West ] et al. Cause-specific mortality of people with Barrett's esophagus compared with the general population: a population-based cohort study. Gastroenterology 2013; 144: 13751383, 1383.e1

[45] Koop $\mathrm{H}$ et al. S2k guideline: gastroesophageal reflux disease guided by the German Society of Gastroenterology: AWMF register no. 021-013. Z Gastroenterol 2014; 52: 1299-1346

[46] Cooper S et al. Risk factors for the development of oesophageal adenocarcinoma in Barrett's oesophagus: a UK primary care retrospective nested case-control study. United European Gastroenterol ] 2014; 2: $91-98$

[47] Nguyen T et al. The Annual Risk of Esophageal Adenocarcinoma Does Not Decrease Over Time in Patients With Barrett's Esophagus. Am J Gastroenterol 2017; 112: 1049-1055

[48] Masclee GM et al. The incidence of Barrett's oesophagus and oesophageal adenocarcinoma in the United Kingdom and The Netherlands is levelling off. Aliment Pharmacol Ther 2014; 39: 1321 - 1330

[49] Kestens C et al. Patients With Barrett's Esophagus and Persistent Lowgrade Dysplasia Have an Increased Risk for High-grade Dysplasia and Cancer. Clin Gastroenterol Hepatol 2016; 14: 956-962.e1 
[50] Steinberg J et al. Koinzidenz von Plattenepithelkarzinomen der Speiseröhre und Kopf-Hals-Karzinomen: Risiko und Früherkennung. Tumor Diagn u Ther 2008; 29: 35-39

[51] Moschler O et al. Chromoendoscopy is a valuable tool for screening of high-risk patients with head and neck cancer for early detection of esophageal cancer. Digestion 2006; 73: 160-166

[52] Ljung R, Martin L, Lagergren J. Oral disease and risk of oesophageal and gastric cancer in a nationwide nested case-control study in Sweden. Eur J Cancer 2011; 47: 2128-2132

[53] Allum WH et al. Guidelines for the management of oesophageal and gastric cancer. Gut 2011; 60: 1449-1472

[54] Rothwell PM et al. Effect of daily aspirin on long-term risk of death due to cancer: analysis of individual patient data from randomised trials. Lancet 2011; 377: 31-41

[55] Algra AM, Rothwell PM. Effects of regular aspirin on long-term cancer incidence and metastasis: a systematic comparison of evidence from observational studies versus randomised trials. Lancet Oncol 2012; 13 : $518-527$

[56] Cuzick J et al. Estimates of benefits and harms of prophylactic use of aspirin in the general population. Ann Oncol 2015; 26: 47-57

[57] Cook NR et al. Alternate-day, low-dose aspirin and cancer risk: long-term observational follow-up of a randomized trial. Ann Intern Med 2013; 159: $77-85$

[58] Cardwell CR et al. Exposure to oral bisphosphonates and risk of cancer. Int J Cancer 2012; 131: E717 -E725

[59] Green J et al. Oral bisphosphonates and risk of cancer of oesophagus, stomach, and colorectum: case-control analysis within a UK primary care cohort. BMJ 2010; 341: c4444

[60] Oh YH, Yoon C, Park SM. Bisphosphonate use and gastrointestinal tract cancer risk: meta-analysis of observational studies. World J Gastroenterol 2012; 18: 5779-5788

[61] Haber SL, McNatty D. An evaluation of the use of oral bisphosphonates and risk of esophageal cancer. Ann Pharmacother 2012; 46: 419-423

[62] Andrici J, Tio M, Eslick GD. Meta-analysis: oral bisphosphonates and the risk of oesophageal cancer. Aliment Pharmacol Ther 2012; 36: 708-716

[63] Busby J et al. The effect of medications which cause inflammation of the gastro-oesophageal tract on cancer risk: a nested case-control study of routine Scottish data. Int J Cancer 2017; 140: 1828-1835

[64] Bjelakovic G et al. Antioxidant supplements for preventing gastrointestinal cancers. Cochrane Database Syst Rev 2008; 3: CD004183

[65] Vigen C, Bernstein L, Wu AH. Occupational physical activity and risk of adenocarcinomas of the esophagus and stomach. Int I Cancer 2006; 118: $1004-1009$

[66] Stehle P, Deutsche Gesellschaft für Ernährung. 12. Ernährungsbericht 2012. European Journal of Nutrition an Food Safety 2012; 4: 2347 - 2564

[67] Liu J et al. Intake of fruit and vegetables and risk of esophageal squamous cell carcinoma: a meta-analysis of observational studies. Int J Cancer 2013; 133: 473-485

[68] Choi Y et al. Consumption of red and processed meat and esophageal cancer risk: metaanalysis. World J Gastroenterol 2013; 19: 1020-1029

[69] Keszei AP et al. Red and processed meat consumption and the risk of esophageal and gastric cancer subtypes in The Netherlands Cohort Study. Ann Oncol 2012; 23: 2319-2326

[70] Huang $W$ et al. Red and processed meat intake and risk of esophageal adenocarcinoma: a meta-analysis of observational studies. Cancer Causes Control 2013; 24: 193-201

[71] Jakszyn P et al. Meat and heme iron intake and esophageal adenocarcinoma in the European Prospective Investigation into Cancer and Nutrition study. Int J Cancer 2013; 133: 2744-2750

[72] Salehi M et al. Meat, fish, and esophageal cancer risk: a systematic review and dose- response meta-analysis. Nutr Rev 2013; 71: 257-267
[73] Palmer JB, Drennan JC, Baba M. Evaluation and treatment of swallowing impairments. Am Fam Physician 2000; 61: 2453-2462

[74] Behrens A et al. How safe is sedation in gastrointestinal endoscopy? A multicentre analysis of 388,404 endoscopies and analysis of data from prospective registries of complications managed by members of the Working Group of Leading Hospital Gastroenterologists (ALGK). Z Gastroenterol 2013; 51: 432-436

[75] Hori $\mathrm{K}$ et al. Lugol-voiding lesions are an important risk factor for a second primary squamous cell carcinoma in patients with esosphageal cancer or head and neck cancer. Am J Gastroenterol 2011; 106: 858 866

[76] Qumseya B] et al. Advanced imaging technologies increase detection of dysplasia and neoplasia in patients with Barrett's esophagus: a metaanalysis and systematic review. Clin Gastroenterol Hepatol 2013; 11: $1562-1570 . e 1-2$

[77] Curvers WL et al. Endoscopic tri-modal imaging is more effective than standard endoscopy in identifying early-stage neoplasia in Barrett's esophagus. Gastroenterology 2010; 139: 1106-1114

[78] Chung CS et al. Image-enhanced endoscopy for detection of second primary neoplasm in patients with esophageal and head and neck cancer: A systematic review and meta-analysis. Head Neck 2016; 38: E2343-E2349

[79] Uedo $\mathrm{N}$ et al. Role of narrow band imaging for diagnosis of early-stage esophagogastric cancer: current consensus of experienced endoscopists in Asia-Pacific region. Dig Endosc 2011; 23: $58-71$

[80] Curvers WL et al. Low-grade dysplasia in Barrett's esophagus: overdiagnosed and underestimated. Am J Gastroenterol 2010; 105: 1523-1530

[81] Sharma P et al. Standard endoscopy with random biopsies versus narrow band imaging targeted biopsies in Barrett's oesophagus: a prospective, international, randomised controlled trial. Gut 2013; 62: 15-21

[82] Canto Ml et al. In vivo endomicroscopy improves detection of Barrett's esophagus-related neoplasia: a multicenter international randomized controlled trial (with video). Gastrointest Endosc 2014; 79: 211-221

[83] Fugazza A et al. Confocal Laser Endomicroscopy in Gastrointestinal and Pancreatobiliary Diseases: A Systematic Review and Meta-Analysis. Biomed Res Int 2016; 2016: 4638683

[84] Gupta A et al. Utility of confocal laser endomicroscopy in identifying high-grade dysplasia and adenocarcinoma in Barrett's esophagus: a systematic review and meta-analysis. Eur ] Gastroenterol Hepatol 2014; 26: $369-377$

[85] Coletta $\mathrm{M}$ et al. Acetic acid chromoendoscopy for the diagnosis of early neoplasia and specialized intestinal metaplasia in Barrett's esophagus: a meta-analysis. Gastrointest Endosc 2016; 83: 57-67.e1

[86] Qumseya B et al. Advanced imaging technologies increase detection of dysplasia and neoplasia in patients with Barrett's esophagus: a metaanalysis and systematic review (Provisional abstract). Clinical Gastroenterology and Hepatology 2013; 11: 1562-1570.e2

[87] Lim $\mathrm{H}$ et al. Clinical significance of early detection of esophageal cancer in patients with head and neck cancer. Gut Liver 2015; 9: 159- 165

[88] Katada C et al. Risk of superficial squamous cell carcinoma developing in the head and neck region in patients with esophageal squamous cell carcinoma. Laryngoscope 2012; 122: 1291 - 1296

[89] Chow TL et al. Prediction of simultaneous esophageal lesions in head and neck squamous cell carcinoma: a multivariate analysis. Arch Otolaryngol Head Neck Surg 2009; 135: $882-885$

[90] Muto $\mathrm{M}$ et al. Association of multiple Lugol-voiding lesions with synchronous and metachronous esophageal squamous cell carcinoma in patients with head and neck cancer. Gastrointest Endosc 2002; 56: $517-521$

[91] Gupta A et al. Utility of confocal laser endomicroscopy in identifying high-grade dysplasia and adenocarcinoma in Barrett's esophagus: a systematic review and meta-analysis (Provisional abstract). Database of Abstracts of Reviews of Effects 2014; 26: 369-377 
[92] Thosani N et al. Diagnostic accuracy of EUS in differentiating mucosal versus submucosal invasion of superficial esophageal cancers: a systematic review and meta-analysis. Gastrointest Endosc 2012; 75: 242 253

[93] Puli SR et al. Staging accuracy of esophageal cancer by endoscopic ultrasound: a metaanalysis and systematic review. World J Gastroenterol 2008; 14: $1479-1490$

[94] van Vliet EP et al. Staging investigations for oesophageal cancer: a meta-analysis. $\mathrm{Br}$ J Cancer 2008; 98: 547 - 557

[95] Tranchemontagne J. Stadification initiale du cancer de l'oesophage: revue systematique sur la performance des methodes diagnostiques. Initial staging of oesophageal cancer: systematic review of the performance of diagnostic methods. Agence d'evaluation des technologies et des modes d'intervention en sante (AETMIS). ETMIS 5(6). Montreal, 2009

[96] Russell I et al. Cancer of oesophagus or gastricus: New assessment of technology of endosonography (COGNATE): Report of pragmatic randomised trial. Health technology assessment 2013; 17: 1 - 13. doi: $10.3310 /$ hta 17390

[97] Luo LN et al. Endoscopic Ultrasound for Preoperative Esophageal Squamous Cell Carcinoma: a Meta-Analysis. PLoS One 2016; 11: e0158373

[98] Takizawa K et al. Lymph node staging in esophageal squamous cell carcinoma: a comparative study of endoscopic ultrasonography versus computed tomography. J Gastroenterol Hepatol 2009; 24: 1687 - 1691

[99] Stiles BM et al. Clinical T2-T3N0M0 esophageal cancer: the risk of node positive disease. Ann Thorac Surg 2011; 92: 491 - 496; discussion 496498

[100] Oldenburg A, Albrecht T. Sonografische Leberdiagnostik bei Tumorpatienten ohne und mit Kontrastmittel. Ultraschall in Med 2008; 29: $488-498$

[101] Kinkel K et al. Detection of hepatic metastases from cancers of the gastrointestinal tract by using noninvasive imaging methods (US, CT, MR imaging, PET): a meta-analysis. Radiology 2002; 224: 748 - 756

[102] Piscaglia F et al. Real time contrast enhanced ultrasonography in detection of liver metastases from gastrointestinal cancer. BMC Cancer 2007; $7: 171$

[103] Dietrich CF et al. Assessment of metastatic liver disease in patients with primary extrahepatic tumors by contrast-enhanced sonography versus CT and MRI. World J Gastroenterol 2006; 12: 1699-1705

[104] Albrecht T et al. Improved detection of hepatic metastases with pulseinversion US during the liver-specific phase of SHU 508A: multicenter study. Radiology 2003; 227: 361 - 370

[105] Hanle MM et al. Screening for liver metastases in women with mammary carcinoma: comparison of contrast-enhanced ultrasound and magnetic resonance imaging. Clin Imaging 2011; 35: 366-370

[106] Seitz K et al. Contrast-Enhanced Ultrasound (CEUS) for the characterization of focal liver lesions prospective comparison in clinical practice: CEUS vs. CT (DEGUM multicenter trial). Parts of this manuscript were presented at the Ultrasound Dreilandertreffen 2008, Davos. Ultraschall in Med 2009; 30: $383-389$

[107] Seitz K et al. Contrast-enhanced ultrasound (CEUS) for the characterization of focal liver lesions in clinical practice (DEGUM Multicenter Trial): CEUS vs. MRI-a prospective comparison in 269 patients. Ultraschall in Med 2010; 31: $492-499$

[108] Strobel D et al. Tumor-specific vascularization pattern of liver metastasis, hepatocellular carcinoma, hemangioma and focal nodular hyperplasia in the differential diagnosis of 1,349 liver lesions in contrastenhanced ultrasound (CEUS). Ultraschall in Med 2009; 30: 376-382

[109] Westwood M et al. Contrast-enhanced ultrasound using SonoVue(R) (sulphur hexafluoride microbubbles) compared with contrast-enhanced computed tomography and contrastenhanced magnetic resonance imaging for the characterisation of focal liver lesions and de- tection of liver metastases: a systematic review and cost-effectiveness analysis. Health Technol Assess 2013; 17: 1-243

[110] Doldi SB et al. Ultrasonographic evaluation of the cervical lymph nodes in preoperative staging of esophageal neoplasms. Abdom Imaging 1998; 23: $275-257$

[111] Lerut T et al. Clinical Practice Guidelines Upper Gastrointestinal Cancer update. 2012

[112] Schreurs LM et al. Current relevance of cervical ultrasonography in staging cancer of the esophagus and gastroesophageal junction. Eur J Radiol 2008; 67: 105-111

[113] Van Overhagen $\mathrm{H}$ et al. Improved assessment of supraclavicular and abdominal metastases in oesophageal and gastro-oesophageal junction carcinoma with the combination of ultrasound and computed tomography. Br J Radiol 1993; 66: $203-208$

[114] van Vliet EP et al. Detection of distant metastases in patients with oesophageal or gastric cardia cancer: a diagnostic decision analysis. $\mathrm{Br}$ ] Cancer 2007; 97: 868-876

[115] Omloo JM et al. Additional value of external ultrasonography of the neck after CT and PET scanning in the preoperative assessment of patients with esophageal cancer. Dig Surg 2009; 26: 43-49

[116] van Overhagen $\mathrm{H}$ et al. Assessment of distant metastases with ultrasound-guided fine-needle aspiration biopsy and cytologic study in carcinoma of the esophagus and gastroesophageal junction. Gastrointest Radiol 1992; 17: 305-310

[117] van Overhagen $\mathrm{H}$ et al. Supraclavicular lymph node metastases in carcinoma of the esophagus and gastroesophageal junction: assessment with CT, US, and US-guided fine-needle aspiration biopsy. Radiology 1991; 179: $155-158$

[118] Mori S et al. Preoperative assessment of resectability for carcinoma of the thoracic esophagus. Part I. Esophagogram and azygogram. Ann Surg 1979; 190: $100-105$

[119] Kumbasar B. Carcinoma of esophagus: radiologic diagnosis and staging. Eur J Radiol 2002; 42: 170 - 180

[120] Fiore D et al. Multimodal imaging of esophagus and cardia cancer before and after treatment. Radiol Med 2006; 111: 804-817

[121] Moreto M. Diagnosis of esophagogastric tumors. Endoscopy 2005; 37 : $26-32$

[122] Kim T] et al. Multimodality assessment of esophageal cancer: preoperative staging and monitoring of response to therapy. Radiographics 2009; 29: $403-421$

[123] Pech O et al. The Impact of Endoscopic Ultrasound and Computed Tomography on the TNM Staging of Early Cancer in Barrett's Esophagus. Am J Gastroenterol 2006; 101: 2223-2229

[124] Quint LE. Thoracic complications and emergencies in oncologic patients. Cancer Imaging 2009; 9 Spec No A: S75 - S82

[125] Makarawo TP et al. Water as a contrast medium: a re-evaluation using the multidetectorrow computed tomography. Am Surg 2013; 79: $728-733$

[126] Kamel IR, Fishman EK. Recent advances in CT imaging of liver metastases. Cancer J 2004; 10: 104-120

[127] Network S.I.G. Scottish Intercollegiate Guidelines Network Management of oesophageal and gastric cancer. A national clinical guideline. 2006

[128] Gollub MJ et al. Pelvic CT in patients with esophageal cancer. Am J Roentgenol 2005; 184: 487-490

[129] Ba-Ssalamah A et al. Accuracy of hydro-multidetector row CT in the local T staging of oesophageal cancer compared to postoperative histopathological results. Eur Radiol 2011; 21: 2326-2335

[130] Takizawa $\mathrm{K}$ et al. Lymph node staging in esophageal squamous cell carcinoma: a comparative study of endoscopic ultrasonography versus computed tomography. J Gastroenterol Hepatol 2009; 24: 1687 - 1691 
[131] Choi j et al. Comparison of endoscopic ultrasonography (EUS), positron emission tomography (PET), and computed tomography (CT) in the preoperative locoregional staging of resectable esophageal cancer. Surg Endosc 2010; 24: 1380-1386

[132] Lowe V] et al. Comparison of positron emission tomography, computed tomography, and endoscopic ultrasound in the initial staging of patients with esophageal cancer. Mol Imaging Biol 2005; 7: 422-430

[133] Heger $U$ et al. Is preoperative chemotherapy followed by surgery the appropriate treatment for signet ring cell containing adenocarcinomas of the esophagogastric junction and stomach? Ann Surg Oncol 2014; 21: $1739-1748$

[134] Blank S et al. A reliable risk score for stage IV esophagogastric cancer. Eur J Surg Oncol 2013; 39: 823-830

[135] Blank S et al. Preoperative therapy of esophagogastric cancer: the problem of nonresponding patients. Langenbecks Arch Surg 2013; 398: $211-220$

[136] Blank S et al. Impact of pretherapeutic routine clinical staging for the individualization of treatment in gastric cancer patients. Langenbecks Arch Surg 2012; 397: 45-55

[137] Sohn KM et al. Comparing MR imaging and CT in the staging of gastric carcinoma. Am J Roentgenol 2000; 174: 1551-1557

[138] Anzidei M et al. Diagnostic performance of 64-MDCT and 1.5-T MRI with high-resolution sequences in the T staging of gastric cancer: a comparative analysis with histopathology. Radiol Med 2009; 114: 1065-1079

[139] Lauenstein TC et al. Whole-body MR imaging: evaluation of patients for metastases. Radiology 2004; 233: 139-148

[140] Wong R, Malthaner R. Esophageal cancer: a systematic review. Curr Probl Cancer 2000; 24: 297-373

[141] Weber MA et al. Assessment of diffusion-weighted MRI and 18F-fluorodeoxyglucose PET/CT in monitoring early response to neoadjuvant chemotherapy in adenocarcinoma of the esophagogastric junction. J Gastrointestin Liver Dis 2013; 22: 45-52

[142] Quint LE, Bogot NR. Staging esophageal cancer. Cancer Imaging 2008; 8 Spec No A: S33-S42

[143] Medical Services Advisory Committee. Positron emission tomography for oesophageal and gastric cancer: assessment report/prepared by the Medical Services Advisory Committee with the assistance of Silke Walleser et al. Australia, 2008

[144] Ballini L et al. Criteria for appropriate use of FDG-PET in esophageal cancer. Dossier 209-2011 ISSN 1591-223X. 2011. 2011 Bologna: Agenzia sanitaria e sociale regionale, Regione Emilia-Romagna (ASSR). ORlentamenti 4

[145] Choi JY et al. Improved detection of individual nodal involvement in squamous cell carcinoma of the esophagus by FDG PET. J Nucl Med 2000; 41: 808-815

[146] Flamen P et al. Utility of positron emission tomography for the staging of patients with potentially operable esophageal carcinoma. J Clin Oncol 2000; 18: 3202-3210

[147] Downey RJ et al. Whole body 18FDG-PET and the response of esophageal cancer to induction therapy: results of a prospective trial. J Clin Oncol 2003; 21: 428-432

[148] Heeren PA et al. Detection of distant metastases in esophageal cancer with (18)F-FDG PET. J Nucl Med 2004; 45: 980 - 987

[149] Noble F et al. Impact of integrated PET/CT in the staging of oesophageal cancer: a UK population-based cohort study. Clin Radiol 2009; 64: 699-705

[150] Kato H, Nakajima M. The efficacy of FDG-PET for the management of esophageal cancer: review article. Ann Thorac Cardiovasc Surg 2012; 18: $412-419$
[151] Hsu WH et al. Positron emission tomography-computed tomography in predicting locoregional invasion in esophageal squamous cell carcinoma. Ann Thorac Surg 2009; 87: $1564-1568$

[152] Barber TW et al. 18F-FDG PET/CT has a high impact on patient management and provides powerful prognostic stratification in the primary staging of esophageal cancer: a prospective study with mature survival data. J Nucl Med 2012; 53: 864-871

[153] Cervino AR et al. Positron emission tomography/computed tomography and esophageal cancer in the clinical practice: How does it affect the prognosis? J Cancer Res Ther 2012; 8: 619-625

[154] Varghese TK Jr et al. The society of thoracic surgeons guidelines on the diagnosis and staging of patients with esophageal cancer. Ann Thorac Surg 2013; 96: 346-356

[155] Findlay JM et al. Pragmatic staging of oesophageal cancer using decision theory involving selective endoscopic ultrasonography, PET and laparoscopy. Br J Surg 2015; 102: 1488-1499

[156] IQWIG. Abschlussbericht Nr.172 zur "Positronen-Emissionstomografie (PET) und (PET/CT) bei Ösophaguskarzinom. 2013

[157] CMS final decision on PET in solid tumors. J Nucl Med 2013; 54: 11n

[158] Osugi $\mathrm{H}$ et al. Bronchoscopic ultrasonography for staging supracarinal esophageal squamous cell carcinoma: impact on outcome. World ] Surg 2003; 27: $590-594$

[159] Wakamatsu T et al. Usefulness of preoperative endobronchial ultrasound for airway invasion around the trachea: esophageal cancer and thyroid cancer. Respiration 2006; 73: 651-657

[160] Omloo JM et al. Value of bronchoscopy after EUS in the preoperative assessment of patients with esophageal cancer at or above the carina. J Gastrointest Surg 2008; 12: 1874-1879

[161] Riedel M et al. Preoperative bronchoscopic assessment of airway invasion by esophageal cancer: a prospective study. Chest 1998; 113 : 687-695

[162] Baisi A, Bonavina L, Peracchia A. Bronchoscopic staging of squamous cell carcinoma of the upper thoracic esophagus. Arch Surg 1999; 134 : $140-143$

[163] Imadahl A et al. Is bronchoscopy a useful additional preoperative examination in esophageal carcinoma? Langenbecks Arch Chir 1990; 375: $326-329$

[164] Nieveen van Dijkum EJ et al. The efficacy of laparoscopic staging in patients with upper gastrointestinal tumors. Cancer 1997; 79: $1315-$ 1359

[165] de Graaf GW et al. The role of staging laparoscopy in oesophagogastric cancers. Eur J Surg Oncol 2007; 33: 988-992

[166] Nath J et al. Peritoneal lavage cytology in patients with oesophagogastric adenocarcinoma. Br J Surg 2008; 95: 721 - 726

[167] Krasna M] et al. Thoracoscopic staging of esophageal cancer: a prospective, multiinstitutional trial. Cancer and Leukemia Group B Thoracic Surgeons. Ann Thorac Surg 1995; 60: 1337-1340

[168] Krasna MJ et al. CALGB 9380: a prospective trial of the feasibility of thoracoscopy/laparoscopy in staging esophageal cancer. Ann Thorac Surg 2001; 71: $1073-1079$

[169] Luketich JD et al. Evaluation of distant metastases in esophageal cancer: 100 consecutive positron emission tomography scans. Ann Thorac Surg 1999; 68: 1133-1136; discussion 1136-1137

[170] Wang GQ et al. Histological precursors of oesophageal squamous cell carcinoma: results from a 13 year prospective follow up study in a high risk population. Gut 2005; 54: 187-192

[171] Kuwano H. Peculiar histopathologic features of esophageal cancer. Surg Today 1998; 28: 573-575

[172] Bosman F et al. WHO Classification of Tumors of the Digestive System. 4th edition Lyon: IARC; 2010 
[173] Schnell TG et al. Long-term nonsurgical management of Barrett's esophagus with highgrade dysplasia. Gastroenterology 2001; 120 : $1607-1619$

[174] Reid BJ et al. Observer variation in the diagnosis of dysplasia in Barrett's esophagus. Hum Pathol 1988; 19: $166-178$

[175] Skacel M et al. The diagnosis of low-grade dysplasia in Barrett's esophagus and its implications for disease progression. Am J Gastroenterol 2000; 95: $3383-3387$

[176] Montgomery E et al. Reproducibility of the diagnosis of dysplasia in Barrett esophagus: a reaffirmation. Hum Pathol 2001; 32: 368 - 378

[177] Ormsby $\mathrm{AH}$ et al. Observer variation in the diagnosis of superficial oesophageal adenocarcinoma. Gut 2002; 51: 671-676

[178] Kerkhof M et al. Grading of dysplasia in Barrett's oesophagus: substantial interobserver variation between general and gastrointestinal pathologists. Histopathology 2007; 50: 920-927

[179] Wani S et al. Greater interobserver agreement by endoscopic mucosal resection than biopsy samples in Barrett's dysplasia. Clin Gastroenterol Hepatol 2010; 8: $783-788$

[180] Weusten B et al. Endoscopic management of Barrett's esophagus: European Society of Gastrointestinal Endoscopy (ESGE) Position Statement. Endoscopy 2017; 49: 191 - 198

[181] Shaheen NJ et al. ACG Clinical Guideline: Diagnosis and Management of Barrett's Esophagus. Am J Gastroenterol 2016; 111: 30 - 50; quiz 51

[182] Bennett C et al. Consensus statements for management of Barrett's dysplasia and earlystage esophageal adenocarcinoma, based on a Delphi process. Gastroenterology 2012; 143: 336 -346

[183] Brown IS, Whiteman DC, Lauwers GY. Foveolar type dysplasia in Barrett esophagus. Mod Pathol 2010; 23: $834-843$

[184] Odze RD. What the gastroenterologist needs to know about the histology of Barrett's esophagus. Curr Opin Gastroenterol 2011; 27: 389 396

[185] Rucker-Schmidt RL et al. Nonadenomatous dysplasia in barrett esophagus: a clinical, pathologic, and DNA content flow cytometric study. Am J Surg Pathol 2009; 33: 886-893

[186] Mahajan D et al. Grading of gastric foveolar-type dysplasia in Barrett's esophagus. Mod Pathol 2010; 23: 1-11

[187] Demicco EG et al. The dichotomy in carcinogenesis of the distal esophagus and esophagogastric junction: intestinal-type vs cardiactype mucosa-associated adenocarcinoma. Mod Pathol 2011; 24: $1177-1190$

[188] Chandrasoma $\mathrm{P}$ et al. A proposal for a new validated histological definition of the gastroesophageal junction. Hum Pathol 2006; 37: $40-47$

[189] Sharma P et al. Dysplasia and cancer in a large multicenter cohort of patients with Barrett's esophagus. Clin Gastroenterol Hepatol 2006; 4: $566-572$

[190] Gatenby PA et al. Treatment modality and risk of development of dysplasia and adenocarcinoma in columnar-lined esophagus. Dis Esophagus 2009; 22: 133 - 142

[191] Weston AP et al. p53 protein overexpression in low grade dysplasia (LGD) in Barrett's esophagus: immunohistochemical marker predictive of progression. Am J Gastroenterol 2001; 96: 1355-1362

[192] Reid BJ et al. Predictors of progression to cancer in Barrett's esophagus: baseline histology and flow cytometry identify low- and high-risk patient subsets. Am J Gastroenterol 2000; 95: 1669-1676

[193] Buttar NS et al. Chemoprevention of esophageal adenocarcinoma by COX-2 inhibitors in an animal model of Barrett's esophagus. Gastroenterology 2002; 122: $1101-1112$

[194] Montgomery E et al. Are ulcers a marker for invasive carcinoma in Barrett's esophagus? Data from a diagnostic variability study with clinical follow-up. Am J Gastroenterol 2002; 97: 27 - 31
[195] Harrison R et al. Detection of intestinal metaplasia in Barrett's esophagus: an observational comparator study suggests the need for a minimum of eight biopsies. Am J Gastroenterol 2007; 102: 1154-1161

[196] Wittekind C. 2010 TNM system: on the 7th edition of TNM classification of malignant tumors. Pathologe 2010; 31: 331-332

[197] Fritz A et al. International Classification of Diseases for oncology (ICD-O). Geneva: WHO. 2000; 3rd ed

[198] Wittekind C, Asamura H, LH S. TNM Atlas. Illustrated guide to the TNM Classification of Malignant Tumours. 6th edition Oxford: Wiley Blackwell; 2014

[199] Wittekind C. TNM-Supplement. Erläuterungen zur einheitlichen Anwendung. 4. Auflage. Wiley- Blackwell [Übersetzung der Englischen Ausgabe: Wittekind Ch, Compton CC, Brierley J, Sobin LH (eds.) TNM Supplement. Commentaries on uniform use. 4th ed.] 2013

[200] Kodama M, Kakegawa T. Treatment of superficial cancer of the eso phagus: a summary of responses to a questionnaire on superficial cancer of the esophagus in Japan. Surgery 1998; 123: 432-429

[201] Bollschweiler E et al. Staging of esophageal carcinoma: length of tumo and number of involved regional lymph nodes. Are these independent prognostic factors? J Surg Oncol 2006; 94: 355-363

[202] Rice TW et al. Esophageal carcinoma: depth of tumor invasion is predictive of regional lymph node status. Ann Thorac Surg 1998; 65: 787 792

[203] Davies AR et al. Tumor stage after neoadjuvant chemotherapy determines survival after surgery for adenocarcinoma of the esophagus and esophagogastric junction. J Clin Oncol 2014; 32: 2983 - 2990

[204] @ C, T.A.H.M.D.C., The American Heritage ${ }^{\circledR}$ Medical Dictionary Copyright (C). Houghton Mifflin Company. 2007

[205] Siewert JR, Stein HJ. Classification of adenocarcinoma of the oesophagogastric junction. Br J Surg 1998; 85: 1457 - 1459

[206] Kato $\mathrm{H}$ et al. Evaluation of the new (1987) TNM classification for thoracic esophageal tumors. Int ] Cancer 1993; 53: 220-223

[207] Enzinger PC, Mayer RJ. Esophageal cancer. N Engl ] Med 2003; 349: $2241-2252$

[208] Gospodarowicz MK, O’Sullivan B, Sobin LH. International Union Against Cancer (UICC). Prognostic Factors in Cancer. 3rd ed. New York: Wiley; 2006

[209] Roder JD et al. Ratio of invaded to removed lymph nodes as a predicto of survival in squamous cell carcinoma of the oesophagus. $\mathrm{Br}$ ] Surg 1994; 81: $410-413$

[210] Hermanek P. Tumors of the gastrointestinal tract and the pancreas: histopathology, staging and prognosis. Anticancer Res 1999; 19: $2393-2396$

[211] Dexter SP et al. Circumferential resection margin involvement: an independent predictor of survival following surgery for oesophageal cancer. Gut 2001; 48: 667-670

[212] Khan OA et al. Prognostic significance of circumferential resection margin involvement following oesophagectomy for cancer. $\mathrm{Br}$ J Cancer 2003; 88: 1549 - 1552

[213] Brucher BL et al. Achalasia and esophageal cancer: incidence, prevalence, and prognosis. World J Surg 2001; 25: 745-749

[214] Sarbia M et al. p53 protein expression and prognosis in squamous cell carcinoma of the esophagus. Cancer 1994; 74: 2218-2223

[215] Mandard AM et al. Pathologic assessment of tumor regression after preoperative chemoradiotherapy of esophageal carcinoma. Clinicopathologic correlations. Cancer 1994; 73: 2680-2686

[216] Becker $\mathrm{K}$ et al. Histomorphology and grading of regression in gastric carcinoma treated with neoadjuvant chemotherapy. Cancer 2003; 98: $1521-1530$

[217] Langer R et al. Prognostic significance of histopathological tumor regression after neoadjuvant chemotherapy in esophageal adenocarcinomas. Mod Pathol 2009; 22: 1555 - 1563 
[218] Becker K et al. Significance of histopathological tumor regression after neoadjuvant chemotherapy in gastric adenocarcinomas: a summary of 480 cases. Ann Surg 2011; 253: 934 - 939

[219] Langer $R$ et al. A multifactorial histopathologic score for the prediction of prognosis of resected esophageal adenocarcinomas after neoadjuvant chemotherapy. Ann Surg Oncol 2014; 21: 915-921

[220] Wu TT et al. Excellent interobserver agreement on grading the extent of residual carcinoma after preoperative chemoradiation in esophageal and esophagogastric junction carcinoma: a reliable predictor for patient outcome. Am J Surg Pathol 2007; 31: 58-64

[221] Mirza A et al. Assessment of Histopathological Response in Gastric and Gastro-Oesophageal Junction Adenocarcinoma following Neoadjuvant Chemotherapy: Which Scoring System to Use? ISRN Pathology 2012; 2012: 8

[222] Karamitopoulou E et al. Assessment of tumor regression of esophageal adenocarcinomas after neoadjuvant chemotherapy: comparison of 2 commonly used scoring approaches. Am J Surg Pathol 2014; 38 : $1551-1556$

[223] Ancona E et al. Only pathologic complete response to neoadjuvant chemotherapy improves significantly the long term survival of patients with resectable esophageal squamous cell carcinoma: final report of a randomized, controlled trial of preoperative chemotherapy versus surgery alone. Cancer 2001; 91: 2165-2174

[224] Rohatgi P et al. Characterization of pathologic complete response after preoperative chemoradiotherapy in carcinoma of the esophagus and outcome after pathologic complete response. Cancer 2005; 104: $2365-2372$

[225] Schneider PM et al. Histomorphologic tumor regression and lymph node metastases determine prognosis following neoadjuvant radiochemotherapy for esophageal cancer: implications for response classification. Ann Surg 2005; 242: 684-692

[226] Meredith KL et al. Pathologic response after neoadjuvant therapy is the major determinant of survival in patients with esophageal cancer. Ann Surg Oncol 2010; 17: 1159-1167

[227] Chirieac LR et al. Posttherapy pathologic stage predicts survival in patients with esophageal carcinoma receiving preoperative chemoradiation. Cancer 2005; 103: 1347-1355

[228] Bang Y] et al. Trastuzumab in combination with chemotherapy versus chemotherapy alone for treatment of HER2-positive advanced gastric or gastro-oesophageal junction cancer (ToGA): a phase 3, open-label, randomised controlled trial. Lancet 2010; 376: 687-697

[229] Bang Y. Pathological features of advanced gastric cancer (GC): Relatationship to human epidermal growth factor receptor 2 (HER2) positivity in the global screening programme of the ToGA trial. J Clin Oncol 2009; 27: 15s. 2009

[230] Satoh T et al. Quality of life in the trastuzumab for gastric cancer trial. Oncologist 2014; 19: $712-719$

[231] Weimann A et al. Significance of preoperative weight loss for perioperative metabolic adaptation and surgical risk in patients with tumors of the upper gastrointestinal tract. Langenbecks Arch Chir 1992; 377 $45-52$

[232] Saito T et al. Factors related to malnutrition in patients with esophageal cancer. Nutrition 1991; 7: 117-121

[233] Falkner D, Plato R, Weimann A. Die Wertigkeit der Feinnadelkatheterjejunostomie in der postoperativen enteralen Ernährung nach Ösophagusresektion. Deutsche Gesellschaft für Chirurgie. 131. Kongress der Dt. Gesellschaft für Chirurgie. Berlin 25.-28.03.2014

[234] van Stijn MF et al. Preoperative nutrition status and postoperative outcome in elderly general surgery patients: a systematic review. JPEN J Parenter Enteral Nutr 2013; 37: 37 - 43

[235] Weimann A et al. Clinical nutrition in surgery. Guidelines of the German Society for Nutritional Medicine. Chirurg 2014; 85: 320-326
[236] Arends et al. S3-Leitlinie der Deutschen Gesellschaft für Ernährungsmedizin. Klinische Ernährung in der nicht-chirurgischen Onkologie. Aktuel Ernährungsmed in press. 2015

[237] Pech O et al. Comparison between endoscopic and surgical resection of mucosal esophageal adenocarcinoma in Barrett's esophagus at two high-volume centers. Ann Surg 2011; 254: 67-72

[238] Prasad GA et al. Endoscopic and surgical treatment of mucosal (T1a) esophageal adenocarcinoma in Barrett's esophagus. Gastroenterology 2009; 137: $815-823$

[239] Ell C et al. Endoscopic mucosal resection of early cancer and high-grade dysplasia in Barrett's esophagus. Gastroenterology 2000; 118: 670677

[240] Ell C et al. Curative endoscopic resection of early esophageal adenocarcinomas (Barrett's cancer). Gastrointest Endosc 2007; 65: 3-10

[241] Pech O et al. Long-term results and risk factor analysis for recurrence after curative endoscopic therapy in 349 patients with high-grade intraepithelial neoplasia and mucosal adenocarcinoma in Barrett's oesophagus. Gut 2008; 57: 1200-1206

[242] Pech O et al. Long-term efficacy and safety of endoscopic resection for patients with mucosal adenocarcinoma of the esophagus. Gastroenterology 2014; 146: $652-660$ e1

[243] Chennat J et al. Complete Barrett's eradication endoscopic mucosal resection: an effective treatment modality for high-grade dysplasia and intramucosal carcinoma-an American singlecenter experience. Am J Gastroenterol 2009; 104: 2684-2692

[244] Moss A et al. Endoscopic resection for Barrett's high-grade dysplasia and early esophageal adenocarcinoma: an essential staging procedure with long-term therapeutic benefit. Am J Gastroenterol 2010; 105: $1276-1283$

[245] Pouw RE et al. Stepwise radical endoscopic resection for eradication of Barrett's oesophagus with early neoplasia in a cohort of 169 patients. Gut 2010; 59: 1169-1177

[246] Pouw RE et al. Efficacy of radiofrequency ablation combined with endoscopic resection for barrett's esophagus with early neoplasia. Clin Gastroenterol Hepatol 2010; 8: $23-29$

[247] van Vilsteren FG et al. Stepwise radical endoscopic resection versus radiofrequency ablation for Barrett's oesophagus with high-grade dysplasia or early cancer: a multicentre randomised trial. Gut 2011; 60: $765-773$

[248] Manner H et al. Early Barrett's carcinoma with "low-risk" submucosal invasion: long-term results of endoscopic resection with a curative intent. Am J Gastroenterol 2008; 103: 2589-2597

[249] Manner $\mathrm{H}$ et al. Efficacy, safety, and long-term results of endoscopic treatment for early stage adenocarcinoma of the esophagus with lowrisk sm1 invasion. Clin Gastroenterol Hepatol 2013; 11: 630-635; quiz e45

[250] Alvarez Herrero L et al. Risk of lymph node metastasis associated with deeper invasion by early adenocarcinoma of the esophagus and cardia: study based on endoscopic resection specimens. Endoscopy 2010; 42: $1030-1036$

[251] Fitzgerald RC et al. British Society of Gastroenterology guidelines on the diagnosis and management of Barrett's oesophagus. Gut 2014; 63: $7-42$

[252] Guo HM et al. Endoscopic submucosal dissection vs endoscopic mucosal resection for superficial esophageal cancer. World J Gastroenterol 2014; 20: $5540-5547$

[253] Neuhaus H et al. Endoscopic submucosal dissection plus radiofrequency ablation of neoplastic Barrett's esophagus. Endoscopy 2012; 44: $1105-1113$

[254] Kagemoto K et al. Clinical outcomes of endoscopic submucosal dissection for superficial Barrett's adenocarcinoma. Gastrointest Endosc 2014; 80: $239-245$ 
[255] Probst A et al. Endoskopische Submukosadissektion (ESD) im Ösophagus. Z Gastroenterol 2013; 51: K233

[256] Feith M, Stein HJ, Siewert JR. Pattern of lymphatic spread of Barrett's cancer. World J Surg 2003; 27: 1052-1057

[257] Holscher AH et al. Prognostic impact of upper, middle, and lower third mucosal or submucosal infiltration in early esophageal cancer. Ann Surg 2011; 254: 802 - 807; discussion 807-808

[258] Zemler B et al. Early Barrett's carcinoma: the depth of infiltration of the tumour correlates with the degree of differentiation, the incidence of lymphatic vessel and venous invasion. Virchows Arch 2010; 456: 609614

[259] Buskens C] et al. Prediction of appropriateness of local endoscopic treatment for high-grade dysplasia and early adenocarcinoma by EUS and histopathologic features. Gastrointest Endosc 2004; 60: 703-710

[260] Westerterp M et al. Outcome of surgical treatment for early adenocarcinoma of the esophagusor gastro-esophageal junction. Virchows Arch 2005; 446: 497-504

[261] Ancona E et al. Prediction of lymph node status in superficial esophageal carcinoma. Ann Surg Oncol 2008; 15: 3278-3288

[262] Liu L et al. Significance of the depth of tumor invasion and lymph node metastasis in suerficially invasive (T1) esophageal adenocarcinoma. Am J Surg Pathol 2005; 29: 1079-1085

[263] Sepesi B et al. Are endoscopic therapies appropriate for superficial submucosal esophageal adenocarcinoma? An analysis of esophagectomy specimens. J Am Coll Surg 2010; 210: 418-427

[264] Badreddine RJ et al. Depth of submucosal invasion does not predict lymph node metastasis and survival of patients with esophageal carcinoma. Clin Gastroenterol Hepatol 2010; 8: 248-253

[265] Griffin SM, Burt AD, Jennings NA. Lymph node metastasis in early esophageal adenocarcinoma. Ann Surg 2011; 254: 731 - 736; discussion $736-737$

[266] Leers JM et al. The prevalence of lymph node metastases in patients with T1 esophageal adenocarcinoma a retrospective review of esophagectomy specimens. Ann Surg 2011; 253: 271-278

[267] Lorenz D et al. Prognostic risk factors of early esophageal adenocarcinomas. Ann Surg 2014; 259: 469-476

[268] May A et al. Local endoscopic therapy for intraepithelial high-grade neoplasia and early adenocarcinoma in Barrett's oesophagus: acutephase and intermediate results of a new treatment approach. Eur ] Gastroenterol Hepatol 2002; 14: 1085-1091

[269] Gossner L et al. Photodynamic ablation of high-grade dysplasia and early cancer in Barrett's esophagus by means of 5-aminolevulinic acid. Gastroenterology 1998; 114: 448-455

[270] Pech $\mathrm{O}$ et al. Long-term results of photodynamic therapy with 5-aminolevulinic acid for superficial Barrett's cancer and high-grade intraepithelial neoplasia. Gastrointest Endosc 2005; 62: 24-30

[271] Overholt BF et al. Five-year efficacy and safety of photodynamic therapy with Photofrin in Barrett's high-grade dysplasia. Gastrointest Endosc 2007; 66: $460-468$

[272] Shaheen NJ et al. Radiofrequency ablation in Barrett's esophagus with dysplasia. N Engl J Med 2009; 360: 2277-2288

[273] Shaheen NJ et al. Durability of radiofrequency ablation in Barrett's esophagus with dysplasia. Gastroenterology 2011; 141: 460-468

[274] Ganz RA et al. Circumferential ablation of Barrett's esophagus that contains high-grade dysplasia: a U.S. Multicenter Registry. Gastrointest Endosc 2008; 68: 35-40

[275] Phoa KN et al. Remission of Barrett's esophagus with early neoplasia 5 years after radiofrequency ablation with endoscopic resection: a Netherlands cohort study. Gastroenterology 2013; 145: 96-104

[276] Orman ES et al. Intestinal metaplasia recurs infrequently in patients successfully treated for Barrett's esophagus with radiofrequency ablation. Am J Gastroenterol 2013; 108: 187 - 195; quiz 196
[277] Van Laethem JL et al. Argon plasma coagulation in the treatment of Barrett's high-grade dysplasia and in situ adenocarcinoma. Endoscopy 2001; 33: 257-261

[278] Ragunath K et al. Endoscopic ablation of dysplastic Barrett's oesophagus comparing argon plasma coagulation and photodynamic therapy: a randomized prospective trial assessing efficacy and cost-effectiveness. Scand J Gastroenterol 2005; 40: 750 - 758

[279] Kelty C] et al. Endoscopic ablation of Barrett's oesophagus: a randomized-controlled trial of photodynamic therapy vs. argon plasma coagulation. Aliment Pharmacol Ther 2004; 20: 1289-1296

[280] Dumot JA et al. An open-label, prospective trial of cryospray ablation for Barrett's esophagus high-grade dysplasia and early esophageal cancer in high-risk patients. Gastrointest Endosc 2009; 70: 635-644

[281] Shaheen NJ et al. Safety and efficacy of endoscopic spray cryotherapy for Barrett's esophagus with high-grade dysplasia. Gastrointest Endosc 2010; 71: 680-685

[282] Ishihara R et al. Comparison of EMR and endoscopic submucosal dissection for en bloc resection of early esophageal cancers in Japan. Gastrointest Endosc 2008; 68: 1066 - 1072

[283] Cao Y et al. Meta-analysis of endoscopic submucosal dissection versus endoscopic mucosal resection for tumors of the gastrointestinal tract. Endoscopy 2009; 41: 751-757

[284] Srivastava A et al. Extent of low-grade dysplasia is a risk factor for the development of esophageal adenocarcinoma in Barrett's esophagus. Am J Gastroenterol 2007; 102: 483 - 493; quiz 694

[285] Phoa KN et al. Radiofrequency ablation vs endoscopic surveillance for patients with Barrett esophagus and low-grade dysplasia: a randomized clinical trial. Jama 2014; 311: 1209-1217

[286] McCann P et al. The safety and effectiveness of endoscopic and non-endoscopic approaches to the management of early esophageal cancer: a systematic review (Structured abstract). Cancer Treatment Reviews 2011; 37: 11-62

[287] Haidry RJ et al. Radiofrequency ablation for early oesophageal squamous neoplasia: outcomes form United Kingdom registry. World J Gastroenterol 2013; 19: 6011-6019

[288] Min BH et al. Feasibility and efficacy of argon plasma coagulation for early esophageal squamous cell neoplasia. Endoscopy 2013; 45: 575578

[289] Tahara K et al. Argon plasma coagulation for superficial esophageal squamous-cell carcinoma in high-risk patients. World J Gastroenterol 2012; 18: 5412-5417

[290] Peters FP et al. Endoscopic treatment of high-grade dysplasia and early stage cancer in Barrett's esophagus. Gastrointest Endosc 2005; 61: $506-514$

[291] Metzger R et al. High volume centers for esophagectomy: what is the number needed to achieve low postoperative mortality? Dis Esophagus 2004; 17: $310-314$

[292] Holscher AH et al. High-volume centers - effect of case load on outcome in cancer surgery. Onkologie 2004; 27: 412-416

[293] Coupland VH et al. Hospital volume, proportion resected and mortality from oesophageal and gastric cancer: a population-based study in England, 2004-2008. Gut 2013; 62: 961-966

[294] Brusselaers N, Mattsson F, Lagergren J. Hospital and surgeon volume in relation to longterm survival after oesophagectomy: systematic review and meta-analysis. Gut 2014; 63: $1393-1400$

[295] Derogar M et al. Hospital and surgeon volume in relation to surviva after esophageal cancer surgery in a population-based study. J Clin Oncol 2013; 31: $551-557$

[296] Reames BN, Shubeck SP, Birkmeyer JD. Strategies for reducing regional variation in the use of surgery: a systematic review. Ann Surg 2014 259: $616-627$ 
[297] Schroder W et al. Preoperative risk analysis-a reliable predictor of postoperative outcome after transthoracic esophagectomy? Langenbecks Arch Surg 2006; 391: 455 -460

[298] Bollschweiler E et al. Preoperative risk analysis in patients with adenocarcinoma or squamous cell carcinoma of the oesophagus. $\mathrm{Br}$ ] Surg 2000; 87: $1106-1110$

[299] Lagarde SM et al. Evaluation of O-POSSUM in predicting in-hospital mortality after resection for oesophageal cancer. Br J Surg 2007; 94: $1521-1526$

[300] Hodari A et al. Assessment of morbidity and mortality after esophagectomy using a modified frailty index. Ann Thorac Surg 2013; 96 : $1240-1245$

[301] Pottgen C, Stuschke M. Radiotherapy versus surgery within multimodality protocols for esophageal cancer - a meta-analysis of the randomized trials. Cancer Treat Rev 2012; 38: 599-604

[302] Markar SR et al. Assessment of short-term clinical outcomes following salvage esophagectomy for the treatment of esophageal malignancy: systematic review and pooled analysis. Ann Surg Oncol 2014; 21: 922 931

[303] Hofstetter WL. Salvage esophagectomy. J Thorac Dis 2014; 6: S341 S349

[304] Wu J et al. Prognostic significance of positive circumferential resection margin in esophageal cancer: a systematic review and meta-analysis. Ann Thorac Surg 2014; 97: 446-453

[305] Holscher AH et al. How safe is high intrathoracic esophagogastrostomy? Chirurg 2003; 74: 726-733

[306] Holscher AH et al. Laparoscopic ischemic conditioning of the stomach for esophageal replacement. Ann Surg 2007; 245: 241 - 246

[307] Li B et al. Comparison of Ivor-Lewis vs Sweet esophagectomy for esophageal squamous cell carcinoma: a randomized clinical trial. JAMA Surg 2015; 150: $292-298$

[308] Schroder W et al. The resection of the azygos vein - necessary or redundant extension of transthoracic esophagectomy? J Gastrointest Surg 2008; 12: 1163-1167

[309] Boone J et al. The effect of azygos vein preservation on mediastinal lymph node harvesting in thoracic esophagolymphadenectomy. Dis Esophagus 2008; 21: $226-229$

[310] Siewert JR et al. Cardia cancer: attempt at a therapeutically relevant classification. Chirurg 1987; 58: 25-32

[311] Kutup A et al. What should be the gold standard for the surgical component in the treatment of locally advanced esophageal cancer: transthoracic versus transhiatal esophagectomy. Ann Surg 2014; 260 : $1016-1022$

[312] Omloo JM et al. Extended transthoracic resection compared with limited transhiatal resection for adenocarcinoma of the mid/distal esophagus: five-year survival of a randomized clinical trial. Ann Surg 2007; 246: 992 -1000; discussion 1000-1001

[313] Workum F et al. Improved Functional Results After Minimally Invasive Esophagectomy: intrathoracic Versus Cervical Anastomosis. Annals of thoracic surgery 2017; 103: 267 - 273. doi:10.1016/j.athoracsur. 2016.07.010

[314] Sasako M et al. Left thoracoabdominal approach versus abdominaltranshiatal approach for gastric cancer of the cardia or subcardia: a randomised controlled trial. Lancet Oncol 2006; 7: 644-651

[315] Kurokawa $Y$ et al. Ten-year follow-up results of a randomized clinical trial comparing left thoracoabdominal and abdominal transhiatal approaches to total gastrectomy for adenocarcinoma of the oesophagogastric junction or gastric cardia. British journal of surgery 2015; 102: 341 - 348. doi:10.1002/bjs.9764

[316] Moehler M et al. German S3-guideline "Diagnosis and treatment of esophagogastric cancer”. Z Gastroenterol 2011; 49: 461 - 531
[317] Peyre CG et al. The number of lymph nodes removed predicts survival in esophageal cancer: an international study on the impact of extent of surgical resection. Ann Surg 2008; 248: 549-556

[318] Peyre CG et al. Predicting systemic disease in patients with esophagea cancer after esophagectomy: a multinational study on the significance of the number of involved lymph nodes. Ann Surg 2008; 248: 979-985

[319] Lerut T et al. Three-field lymphadenectomy for carcinoma of the esophagus and gastroesophageal junction in $174 \mathrm{R} 0$ resections: impact on staging, disease-free survival, and outcome: a plea for adaptation of TNM classification in upper-half esophageal carcinoma. Ann Surg 2004; 240: 962 -972; discussion 972-974

[320] Rizk NP et al. Optimum lymphadenectomy for esophageal cancer. Ann Surg 2010; 251: $46-50$

[321] Fujita $\mathrm{H}$ et al. Optimal lymphadenectomy for squamous cell carcinoma in the thoracic esophagus: comparing the short- and long-term outcome among the four types of lymphadenectomy. World J Surg 2003; 27: $571-579$

[322] Bollschweiler E et al. Influence of neoadjuvant chemoradiation on the number and size of analyzed lymph nodes in esophageal cancer. Ann Surg Oncol 2010; 17: 3187 -3194

[323] Bekkar S et al. The impact of preoperative radiochemotherapy on survival in advanced esophagogastric junction signet ring cell adenocarcinoma. Ann Thorac Surg 2014; 97: 303 - 310

[324] Wittekind C, Meyer HJ. TNM: Klassifikation maligner Tumoren. 7. Auflage Wiley; 2012: 295

[325] Gutschow CA et al. Merendino procedure with preservation of the vagus for early carcinoma of the gastroesophageal junction. Zentralbl Chir 2004; 129: 276-281

[326] Stein $\mathrm{H}$ ] et al. Limited resection for early adenocarcinoma in Barrett's esophagus. Ann Surg 2000; 232: 733-742

[327] Zapletal C et al. Quality of life after surgical treatment of early Barrett's cancer: a prospective comparison of the Ivor-Lewis resection versus the modified Merendino resection. World J Surg 2014; 38: 1444-1452

[328] Schroder W et al. Ivor-Lewis esophagectomy with and without laparoscopic conditioning of the gastric conduit. World J Surg 2010; 34 : $738-743$

[329] Urschel JD et al. Pyloric drainage (pyloroplasty) or no drainage in gastric reconstruction after esophagectomy: a meta-analysis of randomized controlled trials. Dig Surg 2002; 19: 160-164

[330] Gaur P, Swanson SJ. Should we continue to drain the pylorus in patients undergoing an esophagectomy? Dis Esophagus 2014; 27: 568 - 573

[331] Vallbohmer D et al. Diaphragmatic hernia after conventional or laparoscopic-assisted transthoracic esophagectomy. Ann Thorac Surg 2007; 84: $1847-1852$

[332] Price TN et al. A comprehensive review of anastomotic technique in 432 esophagectomies. Ann Thorac Surg 2013; 95: 1154-1160; discussion 1160-1161

[333] Erkmen CP et al. Laparoscopic repair of hiatal hernia after esophagectomy. J Gastrointest Surg 2013; 17: 1370-1374

[334] Avery K et al. The feasibility of a randomized controlled trial of esophagectomy for esophageal cancer - The ROMIO (Randomized Oesophagectomy: Minimally Invasive or Open) study: Protocol for a randomized controlled trial. Trials 2014; 15: doi:10.1186/1745-6215-15-200

[335] Biere SS et al. Minimally invasive versus open oesophagectomy for patients with oesophageal cancer: a multicentre, open-label, randomised controlled trial. Lancet 2012; 379: 1887-1892

[336] Maas KW et al. Quality of Life and Late Complications After Minimally Invasive Compared to Open Esophagectomy: Results of a Randomized Trial. World J Surg 2015; 39: 1986-1993

[337] Straatman ] et al. Minimally Invasive Versus Open Esophageal Resection: Three-year Follow-up of the Previously Reported Randomized Controlled Trial: the TIME Trial. Ann Surg 2017; 266: 232-236 
[338] Luketich JD et al. Outcomes after minimally invasive esophagectomy: review of over 1000 patients. Ann Surg 2012; 256: 95-103

[339] Smithers BM et al. Comparison of the outcomes between open and minimally invasive esophagectomy. Ann Surg 2007; 245: 232 - 240

[340] Briez $\mathrm{N}$ et al. Effects of hybrid minimally invasive oesophagectomy on major postoperative pulmonary complications. Br J Surg 2012; 99: $1547-1553$

[341] Briez $\mathrm{N}$ et al. Is minimally invasive oesophagectomy for cancer decreasing pulmonary complications-Results from a case-control study. J Clin Oncol 2010; 28: 15s

[342] Bonavina L et al. Early outcome of thoracoscopic and hybrid esophagectomy: Propensity-matched comparative analysis. Surgery 2016; 159: $1073-1081$

[343] Straatman J et al. Techniques and short-term outcomes for total minimally invasive Ivor Lewis esophageal resection in distal esophageal and gastroesophageal junction cancers: pooled data from six European centers. Surgical endoscopy and other interventional techniques 2017; 31: 119-126. doi:10.1007/s00464-016-4938-2

[344] Seesing MF] et al. A Propensity Score Matched Analysis of Open Versus Minimally Invasive Transthoracic Esophagectomy in the Netherlands. Ann Surg 2017; 266: 839-846

[345] Schmidt HM et al. Defining Benchmarks for Transthoracic Esophagectomy: A Multicenter Analysis of Total Minimally Invasive Esophagectomy in Low Risk Patients. Ann Surg 2017; 266: 814-821

[346] Bronson NW et al. The incidence of hiatal hernia after minimally invasive esophagectomy. J Gastrointest Surg 2014; 18: 889-893

[347] Willer BL et al. Incidence of diaphragmatic hernias following minimally invasive versus open transthoracic Ivor Lewis McKeown esophagectomy. Hernia 2012; 16: 185-190

[348] Low DE et al. International Consensus on Standardization of Data Collection for Complications Associated With Esophagectomy: Esophagectomy Complications Consensus Group (ECCG). Ann Surg 2015; 262: 286-294

[349] Low DE et al. Benchmarking Complications Associated with Esophagectomy. Ann Surg 2019; 269: 291 - 298

[350] Erhunmwunsee $L$ et al. Impact of pretreatment imaging on survival of esophagectomy after induction therapy for esophageal cancer: who should be given the benefit of the doubt?: esophagectomy outcomes of patients with suspicious metastatic lesions. Ann Surg Oncol 2015; 22: $1020-1025$

[351] Shimoji $\mathrm{H}$ et al. Induction chemotherapy or chemoradiotherapy followed by radical esophagectomy for T4 esophageal cancer: results of a prospective cohort study. World J Surg 2013; 37: 2180-2188

[352] Weimann A et al. ESPEN Guidelines on Enteral Nutrition: Surgery including organ transplantation. Clin Nutr 2006; 25: 224-244

[353] Weimann A, Breitenstein S, Breuer JP et al. S3-Leitlinie der Deutschen Gesellschaft für Ernährungsmedizin: Klinische Ernährung in der Chirurgie. Aktuell Ernährungsmedizin 2013; 38: e155-e197

[354] Kondrup J et al. ESPEN guidelines for nutrition screening 2002. Clin Nutr 2003; 22: $415-421$

[355] Schwegler I et al. Nutritional risk is a clinical predictor of postoperative mortality and morbidity in surgery for colorectal cancer. $\mathrm{Br}$ J Surg 2010; 97: $92-97$

[356] Sorensen J et al. EuroOOPS: an international, multicentre study to implement nutritional risk screening and evaluate clinical outcome. Clin Nutr 2008; 27: 340-349

[357] Kuppinger $D$ et al. Nutritional screening for risk prediction in patients scheduled for abdominal operations. Br J Surg 2012; 99: 728-737

[358] Khuri SF et al. Risk adjustment of the postoperative mortality rate for the comparative assessment of the quality of surgical care: results of the National Veterans Affairs Surgical Risk Study. J Am Coll Surg 1997; 185: $315-327$
[359] Hennessey DB et al. Preoperative hypoalbuminemia is an independent risk factor for the development of surgical site infection following gastrointestinal surgery: a multi-institutional study. Ann Surg 2010; 252: $325-329$

[360] Ligthart-Melis GC et al. Dietician-delivered intensive nutritional support is associated with a decrease in severe postoperative complications after surgery in patients with esophageal cancer. Dis Esophagus 2013; 26: $587-593$

[361] Ellrichmann $\mathrm{M}$ et al. Prospective evaluation of malignant cell seeding after percutaneous endoscopic gastrostomy in patients with oropharyngeal/esophageal cancers. Endoscopy 2013; 45: 526 - 531

[362] Mabvuure NT, Roman A, Khan OA. Enteral immunonutrition versus standard enteral nutrition for patients undergoing oesophagogastric resection for cancer. Int J Surg 2013; 11: 122 -127

[363] Osland E et al. Effect of timing of pharmaconutrition (immunonutrition) administration on outcomes of elective surgery for gastrointestinal malignancies: a systematic review and metaanalysis. JPEN J Parenter Enteral Nutr 2014; 38: 53-69

[364] Jie B et al. Impact of preoperative nutritional support on clinical outcome in abdominal surgical patients at nutritional risk. Nutrition 2012; 28: $1022-1027$

[365] Hill GL. Impact of nutritional support on the clinical outcome of the surgical patient. Clin Nutr 1994; 13: 331 - 340

[366] Burden S et al. Pre-operative nutrition support in patients undergoing gastrointestinal surgery. Cochrane Database Syst Rev 2012; 11: Cd008879

[367] Bozzetti F et al. Perioperative total parenteral nutrition in malnourished, gastrointestinal cancer patients: a randomized, clinical trial. JPEN J Parenter Enteral Nutr 2000; 24: 7-14

[368] Perioperative Total Parenteral Nutrition in Surgical Patients. New England Journal of Medicine 1991; 325: 525-532

[369] Andersen HK, Lewis S], Thomas S. Early enteral nutrition within $24 \mathrm{~h}$ of colorectal surgery versus later commencement of feeding for postoperative complications. Cochrane Database Syst Rev 2006: Cd004080

[370] Lewis S], Andersen HK, Thomas S. Early enteral nutrition within 24h of intestinal surgery versus later commencement of feeding: a systematic review and meta-analysis. J Gastrointest Surg 2009; 13: 569- 575

[371] Osland E et al. Early versus traditional postoperative feeding in patients undergoing resectional gastrointestinal surgery: a meta-analysis. JPEN J Parenter Enteral Nutr 2011; 35: 473-487

[372] Sica GS et al. Needle catheter jejunostomy at esophagectomy for cancer. J Surg Oncol 2005; 91: 276-279

[373] Han-Geurts I] et al. Randomized clinical trial comparing feeding jejunostomy with nasoduodenal tube placement in patients undergoing oesophagectomy. Br J Surg 2007; 94: 31 - 35

[374] Gerritsen A et al. Efficacy and complications of nasojejunal, jejunostomy and parenteral feeding after pancreaticoduodenectomy. J Gastrointest Surg 2012; 16: $1144-1151$

[375] Markides GA, Alkhaffaf B, Vickers ]. Nutritional access routes following oesophagectomy-a systematic review. Eur J Clin Nutr 2011; 65: 565 573

[376] Theologou T et al. The impact of positive circumferential margin on survival following oesophagectomy using the new 7 th TNM classification. Eur J Cardiothorac Surg 2013; 44: 855-859

[377] O'Farrell N] et al. Lack of independent significance of a close ( $<1 \mathrm{~mm})$ circumferential resection margin involvement in esophageal and junctional cancer. Ann Surg Oncol 2013; 20: 2727 - 2733

[378] Fok $\mathrm{M}$ et al. Postoperative radiotherapy for carcinoma of the esophagus: a prospective, randomized controlled study. Surgery 1993; 113: $138-147$

[379] Teniere $P$ et al. Postoperative radiation therapy does not increase survival after curative resection for squamous cell carcinoma of the 
middle and lower esophagus as shown by a multicenter controlled trial. French University Association for Surgical Research. Surg Gynecol Obstet 1991; 173: 123-130

[380] Bao $\mathrm{Y}$ et al. Three-dimensional conformal radiotherapy with concurrent chemotherapy for postoperative recurrence of esophageal squamous cell carcinoma: clinical efficacy and failure pattern. Radiat Oncol 2013; 8: 241

[381] Jingu $\mathrm{K}$ et al. Long-term results of radiotherapy combined with nedaplatin and 5-fluorouracil for postoperative loco-regional recurrent esophageal cancer: update on a phase II study. BMC Cancer 2012; 12 : 542

[382] Ma DY et al. Concurrent three-dimensional conformal radiotherapy and chemotherapy for postoperative recurrence of mediastinal lymph node metastases in patients with esophageal squamous cell carcinoma: a phase 2 single-institution study. Radiat Oncol 2014; 9: 28

[383] Fakhrian K et al. Salvage radiotherapy in patients with recurrent esophageal carcinoma. Strahlenther Onkol 2012; 188: 136-142

[384] Sudo K et al. Locoregional failure rate after preoperative chemoradiation of esophageal adenocarcinoma and the outcomes of salvage strategies. J Clin Oncol 2013; 31: 4306 -4310

[385] Oppedijk V et al. Patterns of recurrence after surgery alone versus preoperative chemoradiotherapy and surgery in the CROSS trials. J Clin Oncol 2014; 32: $385-391$

[386] Malthaner RA et al. Neoadjuvant or adjuvant therapy for resectable esophageal cancer: a systematic review and meta-analysis. BMC Med 2004; $2: 35$

[387] Arnott S] et al. Preoperative radiotherapy for esophageal carcinoma. Cochrane Database Syst Rev 2005: Cd001799

[388] Schwer A et al. Survival effect of neoadjuvant radiotherapy before esophagectomy for patients with esophageal cancer: a surveillance, epidemiology, and end-results study. Int J Radiat Oncol Biol Phys 2009; 73: $449-455$

[389] Ychou M et al. Perioperative chemotherapy compared with surgery alone for resectable gastroesophageal adenocarcinoma: an FNCLCC and FFCD multicenter phase III trial. J Clin Oncol 2011; 29: 1715-1721

[390] Cunningham D et al. Perioperative chemotherapy versus surgery alone for resectable gastroesophageal cancer. N Engl J Med 2006; 355: 11 20

[391] Kelsen DP et al. Chemotherapy followed by surgery compared with surgery alone for localized esophageal cancer. N Engl J Med 1998; 339: 1979- 1984

[392] Boonstra J] et al. Chemotherapy followed by surgery versus surgery alone in patients with resectable oesophageal squamous cell carcinoma: long-term results of a randomized controlled trial. BMC Cancer 2011; 11: 181

[393] Allum WH et al. Long-term results of a randomized trial of surgery with or without preoperative chemotherapy in esophageal cancer. J Clin Oncol 2009; 27: $5062-5067$

[394] Medical Research Council Oesophageal Cancer Working Group. Surgical resection with or without preoperative chemotherapy in oesophageal cancer: a randomised controlled trial. Lancet 2002; 359: 1727 1733

[395] Law S et al. Preoperative chemotherapy versus surgical therapy alone for squamous cell carcinoma of the esophagus: a prospective randomized trial. J Thorac Cardiovasc Surg 1997; 114: 210-217

[396] Al-Batran SE et al. Perioperative chemotherapy with docetaxel, oxaliplatin, and fluorouracil/leucovorin (FLOT) versus epirubicin, cisplatin, and fluorouracil or capecitabine (ECF/ECX) for resectable gastric or gastroesophageal junction (GEJ) adenocarcinoma (FLOT4-AIO): A multicenter, randomized phase 3 trial. Journal of Clinical Oncology 2017; 35: $4004-4004$
[397] Sjoquist KM et al. Survival after neoadjuvant chemotherapy or chemoradiotherapy for resectable oesophageal carcinoma: an updated metaanalysis. Lancet Oncol 2011; 12: 681-692

[398] Markar SR et al. Role of neoadjuvant treatment in clinical T2N0M0 oesophageal cancer: results from a retrospective multi-center European study. Eur J Cancer 2016; 56: 59-68

[399] Speicher P] et al. Induction therapy does not improve survival for clinical stage T2N0 esophageal cancer. J Thorac Oncol 2014; 9: 1195-1201

[400] Crabtree TD et al. Evaluation of the reliability of clinical staging of T2 N0 esophageal cancer: a review of the Society of Thoracic Surgeons database. Ann Thorac Surg 2013; 96: 382-390

[401] Thuss-Patience P, Vecchione L, Keilholz U. Should cT2 esophageal cancer get neoadjuvant treatment before surgery? J Thorac Dis 2017; 9: $2819-2823$

[402] Cunningham D, Allum W, Weeden S. Perioperative chemotherapy in operable gastric and lower oesophageal cancer: a randomised, controlled trial of the UK NCRI Upper GI Clinical Studies Group (the MAGIC trial, ISRCTN 93793971) [abstract]. European journal of cancer 2003; 1 : S18

[403] Clark PI. Medical Research Council (MRC) randomised phase III trial of surgery with or without pre-operative chemotherapy in resectable cancer of the oesophagus. British journal of cancer 2000; 83: 1

[404] Moehler M et al. International comparison of the German evidencebased S3-guidelines on the diagnosis and multimodal treatment of early and locally advanced gastric cancer, including adenocarcinoma of the lower esophagus. Gastric Cancer 2015; 18: 550-563

[405] Fiorica F et al. Preoperative chemoradiotherapy for oesophageal cancer: a systematic review and meta-analysis. Gut 2004; 53: 925 - 930

[406] Burmeister BH et al. Surgery alone versus chemoradiotherapy followed by surgery for resectable cancer of the oesophagus: a randomised controlled phase III trial. Lancet Oncol 2005; 6: 659-668

[407] Lee JL et al. A single institutional phase III trial of preoperative chemotherapy with hyperfractionation radiotherapy plus surgery versus surgery alone for resectable esophageal squamous cell carcinoma. Ann Oncol 2004; 15: 947 - 954

[408] Bosset JF et al. Chemoradiotherapy followed by surgery compared with surgery alone in squamous-cell cancer of the esophagus. N Engl J Med 1997; 337: $161-167$

[409] Tepper ] et al. Phase III trial of trimodality therapy with cisplatin, fluorouracil, radiotherapy, and surgery compared with surgery alone for esophageal cancer: CALGB 9781. J Clin Oncol 2008; 26: 1086-1092

[410] Stahl M et al. Phase III comparison of preoperative chemotherapy compared with chemoradiotherapy in patients with locally advanced adenocarcinoma of the esophagogastric junction. J Clin Oncol 2009; 27: $851-816$

[411] van Hagen P et al. Preoperative chemoradiotherapy for esophageal or junctional cancer. N Engl J Med 2012; 366: 2074-2084

[412] Urba SG et al. Randomized trial of preoperative chemoradiation versus surgery alone in patients with locoregional esophageal carcinoma. J Clin Oncol 2001; 19: 305-313

[413] Conroy T et al. Definitive chemoradiotherapy with FOLFOX versus fluorouracil and cisplatin in patients with oesophageal cancer (PRODIGE5/ ACCORD17): final results of a randomised, phase 2/3 trial. Lancet Oncol 2014; 15: $305-314$

[414] van Hagen P et al. Preoperative chemoradiotherapy for esophageal or junctional cancer. N Engl J Med 2012; 366: 2074 - 2084

[415] Herskovic A et al. Combined chemotherapy and radiotherapy compared with radiotherapy alone in patients with cancer of the esophagus. N Engl J Med 1992; 326: 1593 - 1598

[416] Cao XF et al. Effects of neoadjuvant radiochemotherapy on pathological staging and prognosis for locally advanced esophageal squamous cell carcinoma. Dis Esophagus 2009; 22: $477-481$ 
[417] Walsh TN et al. A comparison of multimodal therapy and surgery for esophageal adenocarcinoma. N Engl J Med 1996; 335: 462-467

[418] Walsh TN, Grannell M, Mansoor S. Predictive factors for success of neo-adjuvant therapy in upper gastrointestinal cancer. J Gastroenterol Hepatol 2002; 17: S172-S175

[419] Walsh TN et al. Neoadjuvant treatment of advanced stage esophageal adenocarcinoma increases survival. Dis Esophagus 2002; 15: 121 - 124

[420] Apinop C, Puttisak P, Preecha N. A prospective study of combined therapy in esophageal cancer. Hepatogastroenterology 1994; 41: 391 393

[421] Le Prise E et al. A randomized study of chemotherapy, radiation therapy, and surgery versus surgery for localized squamous cell carcinoma of the esophagus. Cancer 1994; 73: 1779-1784

[422] Kumagai K et al. Meta-analysis of postoperative morbidity and perioperative mortality in patients receiving neoadjuvant chemotherapy or chemoradiotherapy for resectable oesophageal and gastro-oesophageal junctional cancers. Br J Surg 2014; 101: 321 - 338

[423] Ronellenfitsch U et al. Perioperative chemo(radio)therapy versus primary surgery for resectable adenocarcinoma of the stomach, gastroesophageal junction, and lower esophagus. Cochrane Database of Systematic Reviews 2013. doi:10.1002/14651858.CD008107.pub2

[424] Lone GN et al. Role of preoperative chemotherapy in squamous cell carcinoma of esophagus in kashmir, a cancer belt a pilot study. Asian Pac J Cancer Prev 2011; 12: 465-470

[425] Zhao Y et al. Perioperative versus Preoperative Chemotherapy with Surgery in Patients with Resectable Squamous Cell Carcinoma of Esophagus: A Phase III Randomized Trial. Journal of thoracic oncology: official publication of the International Association for the Study of Lung Cancer 2015; 10: 1349-1356. doi:10.1097/JTO.0000000000000612

[426] Al-Batran SE et al. Histopathological regression after neoadjuvant docetaxel, oxaliplatin, fluorouracil, and leucovorin versus epirubicin, cisplatin, and fluorouracil or capecitabine in patients with resectable gastric or gastro-oesophageal junction adenocarcinoma (FLOT4-AIO): results from the phase 2 part of a multicentre, open-label, randomised phase 2/3 trial. Lancet Oncol 2016; 17: 1697 - 1708

[427] Sjoquist KM et al. Survival after neoadjuvant chemotherapy or chemoradiotherapy for resectable oesophageal carcinoma: an updated metaanalysis. Lancet Oncol 2011; 12: 681-692

[428] Kranzfelder M et al. Meta-analysis of neoadjuvant treatment modalities and definitive non-surgical therapy for oesophageal squamous cell cancer. Br J Surg 2011; 98: $768-783$

[429] Ando $\mathrm{N}$ et al. A randomized trial comparing postoperative adjuvant chemotherapy with cisplatin and 5-fluorouracil versus preoperative chemotherapy for localized advanced squamous cell carcinoma of the thoracic esophagus (JCOG9907). Ann Surg Oncol 2012; 19: 68-74

[430] Lordick F. Optimizing neoadjuvant chemotherapy through the use of early response evaluation by positron emission tomography. Recent Results Cancer Res 2012; 196: 201 - 211

[431] Weber WA et al. Prediction of response to preoperative chemotherapy in adenocarcinomas of the esophagogastric junction by metabolic imaging. J Clin Oncol 2001; 19: 3058-3065

[432] Ott $\mathrm{K}$ et al. Metabolic imaging predicts response, survival, and recurrence in adenocarcinomas of the esophagogastric junction. J Clin Oncol 2006; 24: $4692-4698$

[433] Ilson DH. Cancer of the gastroesophageal junction: combined modality therapy. Surg Oncol Clin N Am 2006; 15: 803 - 824

[434] Wieder HA et al. Comparison of changes in tumor metabolic activity and tumor size during chemotherapy of adenocarcinomas of the esophagogastric junction. J Nucl Med 2005; 46: 2029-2034

[435] Lordick F et al. PET to assess early metabolic response and to guide treatment of adenocarcinoma of the oesophagogastric junction: the MUNICON phase II trial. Lancet Oncol 2007; 8: 797 - 805
[436] zum Buschenfelde CM et al. (18)F-FDG PET-guided salvage neoadjuvant radiochemotherapy of adenocarcinoma of the esophagogastric junction: the MUNICON II trial. J Nucl Med 2011; 52: 1189-1196

[437] Gillham CM et al. (18)FDG uptake during induction chemoradiation for oesophageal cancer fails to predict histomorphological tumour response. Br J Cancer 2006; 95: 1174-1179

[438] Klaeser B et al. Limited predictive value of FDG-PET for response assessment in the preoperative treatment of esophageal cancer: results of a prospective multi-center trial (SAKK 75/02). Onkologie 2009; 32: $724-730$

[439] Malik V et al. Early repeated 18F-FDG PET scans during neoadjuvant chemoradiation fail to predict histopathologic response or survival benefit in adenocarcinoma of the esophagus. J Nucl Med 2010; 51 : $1863-1869$

[440] van Heijl M et al. Fluorodeoxyglucose positron emission tomography for evaluating early response during neoadjuvant chemoradiotherapy in patients with potentially curable esophageal cancer. Ann Surg 2011; 253: $56-63$

[441] Chang DT et al. Treatment of esophageal cancer based on histology: a surveillance epidemiology and end results analysis. Am J Clin Oncol 2009; 32: $405-410$

[442] Karran A et al. Propensity score analysis of oesophageal cancer treatment with surgery or definitive chemoradiotherapy. Br J Surg 2014; 101: $502-510$

[443] al-Sarraf M et al. Progress report of combined chemoradiotherapy versus radiotherapy alone in patients with esophageal cancer: an intergroup study. J Clin Oncol 1997; 15: 277 - 284

[444] Crehange G et al. Phase III trial of protracted compared with splitcourse chemoradiation for esophageal carcinoma: Federation Francophone de Cancerologie Digestive 9102. J Clin Oncol 2007; 25: 4895 4901

[445] Kawaguchi $Y$ et al. Patterns of failure associated with involved field radiotherapy in patients with clinical stage I thoracic esophageal cancer. Jpn J Clin Oncol 2011; 41: 1007-1012

[446] Wong R, Malthaner R. Combined chemotherapy and radiotherapy (without surgery) compared with radiotherapy alone in localized carcinoma of the esophagus. Cochrane Database Syst Rev 2006: Cd002092

[447] Yamada K et al. Treatment results of chemoradiotherapy for clinical stage I (T1N0M0) esophageal carcinoma. Int ] Radiat Oncol Biol Phys 2006; 64: $1106-1111$

[448] Kat H et al. A phase II trial of chemoradiotherapy for stage I esophageal squamous cell carcinoma: Japan Clinical Oncology Group Study (JCOG9708). Jpn J Clin Oncol 2009; 39: 638-643

[449] Kuwano $\mathrm{H}$ et al. Guidelines for diagnosis and treatment of carcinoma of the esophagus. Esophagus 2008; 5: $117-132$

[450] Gkika E et al. Long-term results of definitive radiochemotherapy in locally advanced cancers of the cervical esophagus. Dis Esophagus 2014; 27: $678-684$

[451] Grass GD et al. Cervical esophageal cancer: A population-based study. Head Neck 2015; 37: 808-814

[452] Burmeister BH et al. Thirty-four patients with carcinoma of the cervical esophagus treated with chemoradiation therapy. Arch Otolaryngol Head Neck Surg 2000; 126: 205-208

[453] Ajani JA et al. Esophageal and esophagogastric junction cancers. J Natl Compr Canc Netw 2011; 9: 830-887

[454] Gao X. Treatment guideline of radiotherapy for Chinese esophageal carcinoma (draft). Chin J Cancer 2010; 29: 855-859

[455] Fenkell L et al. Dosimetric comparison of IMRT vs. 3D conformal radiotherapy in the treatment of cancer of the cervical esophagus. Radiother Oncol 2008; 89: 287-291 
[456] Ma JB et al. Feasibility of involved-field conformal radiotherapy for cervical and upper-thoracic esophageal cancer. Onkologie 2011; 34 : $599-604$

[457] Ma JB et al. Feasibility of Involved-Field Conformal Radiotherapy for Cervical and Upper- Thoracic Esophageal Cancer. Oncology Research and Treatment 2011; 34: 599-604

[458] Tai P et al. Improving the consistency in cervical esophageal target volume definition by special training. Int J Radiat Oncol Biol Phys 2002; 53: $766-774$

[459] Ott K et al. Limited resection and free jejunal graft interposition for squamous cell carcinoma of the cervical oesophagus. Br J Surg 2009; 96: $258-266$

[460] Panhofer $P$ et al. Influence of resection extent on morbidity in surgery for squamous cell cancer at the pharyngoesophageal junction. Langenbecks Arch Surg 2013; 398: 221 - 230

[461] Kranzfelder $M$ et al. Littoral cell angioma and angiosarcoma of the spleen: report of two cases in siblings and review of the literature. J Gastrointest Surg 2012; 16: 863 -867

[462] Bedenne $L$ et al. Chemoradiation followed by surgery compared with chemoradiation alone in squamos cancer of the esophagus: FFCD 9102. J Clin Oncol 2007; 25: $1160-1168$

[463] Minsky BD et al. INT 0123 (Radiation Therapy Oncology Group 94-05) phase III trial of combined-modality therapy for esophageal cancer: high-dose versus standard-dose radiation therapy. J Clin Oncol 2002; 20: $1167-1174$

[464] Ajani JA et al. Esophageal and esophagogastric junction cancers. J Natl Compr Canc Netw 2011; 9: 830-887

[465] Crosby T et al. Chemoradiotherapy with or without cetuximab in patients with oesophageal cancer (SCOPE1): a multicentre, phase 2/3 randomised trial. Lancet Oncol 2013; 14: 627-637

[466] Meerten E et al. Definitive concurrent chemoradiation (CRT) with weekly paclitaxel and carboplatin for patients (pts) with irresectable esophageal cancer: A phase II study. in ASCO Annual Meeting Proceedings 2010

[467] Suntharalingam M et al. The Initial Report of Local Control on RTOG 0436: A Phase 3 Trial Evaluating the Addition of Cetuximab to Paclitaxel, Cisplatin, and Radiation for Patients With Esophageal Cancer Treated Without Surgery. International Journal of Radiation Oncology Biology Physics 2014; 90: S3

[468] Stahl M et al. Chemoradiation with and without surgery in patients with locally advanced squamous cell carcinoma of the esophagus. J Clin Oncol 2005; 23: 2310-2317

[469] Kole TP et al. Comparison of heart and coronary artery doses associated with intensity-modulated radiotherapy versus three-dimensional conformal radiotherapy for distal esophageal cancer. Int J Radiat Oncol Biol Phys 2012; 83: 1580-1586

[470] Lee HK et al. Postoperative pulmonary complications after preoperative chemoradiation for esophageal carcinoma: correlation with pulmonary dose-volume histogram parameters. Int J Radiat Oncol Biol Phys 2003; 57: $1317-1322$

[471] Ruol A et al. Interval between neoadjuvant chemoradiotherapy and surgery for squamous cell carcinoma of the thoracic esophagus: does delayed surgery have an impact on outcome? Ann Surg 2010; 252: $788-796$

[472] Kim JY et al. Does the timing of esophagectomy after chemoradiation affect outcome? Ann Thorac Surg 2012; 93: 207 - 212; discussion 212213

[473] Tachimori Y. Role of salvage esophagectomy after definitive chemoradiotherapy. Gen Thorac Cardiovasc Surg 2009; 57: 71 - 78

[474] Tomimaru Y et al. Factors affecting the prognosis of patients with esophageal cancer undergoing salvage surgery after definitive chemoradiotherapy. J Surg Oncol 2006; 93: 422-428
[475] Marks JL et al. Salvage esophagectomy after failed definitive chemoradiation for esophageal adenocarcinoma. Ann Thorac Surg 2012; 94 : 1126-1132; discussion 1132-1133

[476] Taketa T et al. Propensity-based matching between esophagogastric cancer patients who had surgery and who declined surgery after preoperative chemoradiation. Oncology 2013; 85: 95-99

[477] Ariga $\mathrm{H}$ et al. Prospective comparison of surgery alone and chemoradiotherapy with selective surgery in resectable squamous cell carcinoma of the esophagus. Int J Radiat Oncol Biol Phys 2009; 75: 348 - 356

[478] Zheng B et al. Role of adjuvant chemoradiotherapy in treatment of resectable esophageal carcinoma: a meta-analysis. Chin Med J (Engl) 2013; 126: $1178-1182$

[479] Thallinger CM et al. Pre- and postoperative treatment modalities for esophageal squamous cell carcinoma. Anticancer Res 2012; 32: 4609 4627

[480] Xu Y et al. Neoadjuvant versus adjuvant treatment: which one is better for resectable esophageal squamous cell carcinoma? World J Surg Oncol 2012; 10: 173

[481] Lv J et al. Long-term efficacy of perioperative chemoradiotherapy on esophageal squamous cell carcinoma. World J Gastroenterol 2010; 16 : $1649-1654$

[482] Cao XF et al. A prospective comparison between surgery alone and postoperative chemoradiotherapy for locally advanced esophageal squamous cell carcinoma. Zhonghua Zhong Liu Za Zhi 2010; 32: 452 455

[483] Ohri $\mathrm{N}$ et al. Who benefits from adjuvant radiation therapy for gastric cancer? A meta-analysis. Int J Radiat Oncol Biol Phys 2013; 86: 330 335

[484] Bamias A et al. A randomized phase III study of adjuvant platinum/ docetaxel chemotherapy with or without radiation therapy in patients with gastric cancer. Cancer Chemother Pharmacol 2010; 65: 10091021

[485] Lee J et al. Phase III trial comparing capecitabine plus cisplatin versus capecitabine plus cisplatin with concurrent capecitabine radiotherapy in completely resected gastric cancer with D2 lymph node dissection: the ARTIST trial. J Clin Oncol 2012; 30: 268-273

[486] Macdonald JS et al. Chemoradiotherapy after surgery compared with surgery alone for adenocarcinoma of the stomach or gastroesophageal junction. N Engl J Med 2001; 345: 725 -730

[487] Kwon HC et al. Adjuvant chemoradiation versus chemotherapy in completely resected advanced gastric cancer with D2 nodal dissection. Asia Pac J Clin Oncol 2010; 6: 278-285

[488] Paoletti X et al. Benefit of adjuvant chemotherapy for resectable gastric cancer: a meta-analysis. Jama 2010; 303: 1729-1737

[489] Abate E et al. Recurrence after esophagectomy for adenocarcinoma: defining optimal follow-up intervals and testing. J Am Coll Surg 2010; 210: $428-435$

[490] Mantziari S et al. Gastroesophageal cancer: an update on diagnosis and treatment. Rev Med Suisse 2014; 10: 1331 - 1336

[491] Ouattara M et al. Body mass index kinetics and risk factors of malnutrition one year after radical oesophagectomy for cancer. Eur J Cardiothorac Surg 2012; 41: 1088-1093

[492] Haverkort EB et al. Suboptimal intake of nutrients after esophagectomy with gastric tube reconstruction. J Acad Nutr Diet 2012; 112: 1080 1087

[493] Ryan AM et al. Post-oesophagectomy early enteral nutrition via a needle catheter jejunostomy: 8-year experience at a specialist unit. Clin Nutr 2006; 25: 386-393

[494] Mishra SI et al. Exercise interventions on health-related quality of life for people with cancer during active treatment. Cochrane Database Syst Rev 2012; 8: Cd008465 
[495] Bourke L et al. Interventions to improve exercise behaviour in sedentary people living with and beyond cancer: a systematic review. $\mathrm{Br}$ J Cancer 2014; 110: 831 - 841

[496] Rock CL et al. Nutrition and physical activity guidelines for cancer survivors. CA Cancer J Clin 2012; 62: 243 -274

[497] Mishra SI et al. Exercise interventions on health-related quality of life for cancer survivors. Cochrane Database Syst Rev 2012; 8: Cd007566

[498] Cunningham D et al. Capecitabine and oxaliplatin for advanced esophagogastric cancer. N Engl J Med 2008; 358: 36-46

[499] Webb A et al. Randomized trial comparing epirubicin, cisplatin, and fluorouracil versus fluorouracil, doxorubicin, and methotrexate in advanced esophagogastric cancer. J Clin Oncol 1997; 15: 261 - 267

[500] Ross P et al. Prospective randomized trial comparing mitomycin, cisplatin, and protracted venous-infusion fluorouracil (PVI 5-FU) With epirubicin, cisplatin, and PVI 5-FU in advanced esophagogastric cancer. J Clin Oncol 2002; 20: 1996-2004

[501] Van Cutsem E et al. Phase III study of docetaxel and cisplatin plus fluorouracil compared with cisplatin and fluorouracil as first-line therapy for advanced gastric cancer: a report of the V325 Study Group. J Clin Oncol 2006; 24: 4991 - 4997

[502] Ajani JA et al. Multicenter phase III comparison of cisplatin/S-1 with cisplatin/infusional fluorouracil in advanced gastric or gastroesophageal adenocarcinoma study: the FLAGS trial. J Clin Oncol 2010; 28: $1547-1553$

[503] Al-Batran SE et al. Phase III trial in metastatic gastroesophageal adenocarcinoma with fluorouracil, leucovorin plus either oxaliplatin or cisplatin: a study of the Arbeitsgemeinschaft Internistische Onkologie. J Clin Oncol 2008; 26: 1435-1442

[504] Kang YK et al. Capecitabine/cisplatin versus 5-fluorouracil/cisplatin as first-line therapy in patients with advanced gastric cancer: a randomised phase III noninferiority trial. Ann Oncol 2009; 20: 666-673

[505] Okines AF et al. Meta-analysis of the REAL-2 and ML17032 trials: evaluating capecitabine-based combination chemotherapy and infused 5-fluorouracil-based combination chemotherapy for the treatment of advanced oesophago-gastric cancer. Ann Oncol 2009; 20: 1529 - 1534

[506] Lutz MP et al. Weekly infusional high-dose fluorouracil (HD-FU), HD-FU plus folinic acid (HD- FU/FA), or HD-FU/FA plus biweekly cisplatin in advanced gastric cancer: randomized phase II trial 40953 of the European Organisation for Research and Treatment of Cancer Gastrointestinal Group and the Arbeitsgemeinschaft Internistische Onkologie. J Clin Oncol 2007; 25: 2580-2585

[507] Al-Batran SE et al. Biweekly fluorouracil, leucovorin, oxaliplatin, and docetaxel (FLOT) for patients with metastatic adenocarcinoma of the stomach or esophagogastric junction: a phase II trial of the Arbeitsgemeinschaft Internistische Onkologie. Ann Oncol 2008; 19: 1882 - 1887

[508] NCCN Clinical Practice Guidelines in Oncology: Esophageal and Esophagastric Junction Cancers. National Comprehensive Cancer Network. 2011

[509] Xiang XJ et al. A phase II study of capecitabine plus oxaliplatin as firstline chemotherapy in elderly patients with advanced gastric cancer. Chemotherapy 2012; 58: $1-7$

[510] Catalano V et al. A phase II study of modified FOLFOX as first-line chemotherapy for metastatic gastric cancer in elderly patients with associated diseases. Gastric Cancer 2013; 16: 411 -419

[511] Al-Batran SE et al. The feasibility of triple-drug chemotherapy combination in older adult patients with oesophagogastric cancer: a randomised trial of the Arbeitsgemeinschaft Internistische Onkologie (FLOT65+). Eur J Cancer 2013; 49: 835-842

[512] Hall PS et al. A randomised phase II trial and feasibility study of palliative chemotherapy in frail or elderly patients with advanced gastroesophageal cancer (321GO). Br J Cancer 2017; 116: 472 - 478

[513] Grunberger B et al. Palliative chemotherapy for recurrent and metastatic esophageal cancer. Anticancer Res 2007; 27: 2705 - 2714
[514] NCCN practice guidelines for the management of psychosocial distress. National Comprehensive Cancer Network. 1999

[515] Adam R et al. Hepatic resection for noncolorectal nonendocrine liver metastases: analysis of 1,452 patients and development of a prognostic model. Ann Surg 2006; 244: $524-535$

[516] Shiono S et al. Disease-free interval length correlates to prognosis of patients who underwent metastasectomy for esophageal lung metastases. J Thorac Oncol 2008; 3: 1046 - 1049

[517] Ichikawa $\mathrm{H}$ et al. Operative treatment for metachronous pulmonary metastasis from esophageal carcinoma. Surgery 2011; 149: 164 - 170

[518] Ichida $\mathrm{H}$ et al. Pattern of postoperative recurrence and hepatic and/or pulmonary resection for liver and/or lung metastases from esophageal carcinoma. World J Surg 2013; 37: 398-407

[519] Miyata $\mathrm{H}$ et al. Salvage esophagectomy after definitive chemoradiotherapy for thoracic esophageal cancer. J Surg Oncol 2009; 100: 442 446

[520] Morita M et al. Clinical significance of salvage esophagectomy for remnant or recurrent cancer following definitive chemoradiotherapy. J Gastroenterol 2011; 46: 1284-1291

[521] Schieman C et al. Salvage resections for recurrent or persistent cancer of the proximal esophagus after chemoradiotherapy. Ann Thorac Surg 2013; 95: $459-463$

[522] Thuss-Patience PC et al. Survival advantage for irinotecan versus best supportive care as second-line chemotherapy in gastric cancer - a randomised phase III study of the Arbeitsgemeinschaft Internistische Onkologie (AIO). Eur J Cancer 2011; 47: 2306-2314

[523] Hironaka S et al. Randomized, open-label, phase III study comparing irinotecan with paclitaxel in patients with advanced gastric cancer without severe peritoneal metastasis after failure of prior combination chemotherapy using fluoropyrimidine plus platinum: WJOG 4007 trial. J Clin Oncol 2013; 31: 4438 -4444

[524] Ford HE et al. Docetaxel versus active symptom control for refractory oesophagogastric adenocarcinoma (COUGAR-02): an open-label, phase 3 randomised controlled trial. Lancet Oncol 2014; 15: 78-86

[525] Fuchs CS et al. Ramucirumab monotherapy for previously treated advanced gastric or gastro-oesophageal junction adenocarcinoma (REGARD): an international, randomised, multicentre, placebo-controlled, phase 3 trial. Lancet 2014; 383: 31 - 39

[526] Wilke $\mathrm{H}$ et al. Ramucirumab plus paclitaxel versus placebo plus paclitaxel in patients with previously treated advanced gastric or gastro-oesophageal junction adenocarcinoma (RAINBOW): a doubleblind, randomised phase 3 trial. Lancet Oncol 2014; 15: 1224-1235

[527] Thallinger CM, Raderer M, Hejna M. Esophageal cancer: a critical evaluation of systemic second-line therapy. J Clin Oncol 2011; 29: 47094714

[528] Thuss-Patience PC et al. Trastuzumab emtansine versus taxane use for previously treated HER2-positive locally advanced or metastatic gastric or gastro-oesophageal junction adenocarcinoma (GATSBY): an international randomised, open-label, adaptive, phase 2/3 study. Lancet Oncol 2017; 18: 640-653

[529] Amdal CD et al. Patient-reported outcomes evaluating palliative radiotherapy and chemotherapy in patients with oesophageal cancer: a systematic review. Acta Oncol 2013; 52: 679-690

[530] Sgourakis G et al. Survival after chemotherapy and/or radiotherapy versus self-expanding metal stent insertion in the setting of inoperable esophageal cancer: a case-control study. BMC Cancer 2012; 12: 70

[531] Javed A et al. Palliative stenting with or without radiotherapy for inoperable esophageal carcinoma: a randomized trial. J Gastrointest Cancer 2012; 43: 63-69

[532] Amdal CD et al. Palliative brachytherapy with or without primary stent placement in patients with oesophageal cancer, a randomised phase III trial. Radiother Oncol 2013; 107: 428-433 
[533] Homs MY et al. Single-dose brachytherapy versus metal stent placement for the palliation of dysphagia from oesophageal cancer: multicentre randomised trial. Lancet 2004; 364: 1497-1504

[534] Rosenblatt E et al. Adding external beam to intra-luminal brachytherapy improves palliation in obstructive squamous cell oesophageal cancer: a prospective multi-centre randomized trial of the International Atomic Energy Agency. Radiother Oncol 2010; 97: 488-494

[535] Sgourakis G et al. The use of self-expanding stents in esophageal and gastroesophageal junction cancer palliation: a meta-analysis and metaregression analysis of outcomes. Dig Dis Sci 2010; 55: 3018-3030

[536] Sabharwal T et al. A randomised prospective comparison of the Flamingo Wallstent and Ultraflex stent for palliation of dysphagia associated with lower third oesophageal carcinoma. Gut 2003; 52: 922 926

[537] Shenfine J et al. A pragmatic randomised controlled trial of the costeffectiveness of palliative therapies for patients with inoperable oesophageal cancer. Health Technol Assess 2005; 9: 1 - 121

[538] Vakil $\mathrm{N}$ et al. A prospective, randomized, controlled trial of covered expandable metal stents in the palliation of malignant esophageal obstruction at the gastroesophageal junction. Am J Gastroenterol 2001; 96: 1791 - 1796

[539] Miyayama S et al. Malignant esophageal stricture and fistula: palliative treatment with polyurethane-covered Gianturco stent. J Vasc Interv Radiol 1995; 6: 243-248

[540] Alexander EP et al. Evolving management and outcome of esophageal cancer with airway involvement. Ann Thorac Surg 2001; 71: 1640 1644

[541] Conio $\mathrm{M}$ et al. A randomized prospective comparison of self-expandable plastic stents and partially covered self-expandable metal stents in the palliation of malignant esophageal dysphagia. Am J Gastroenterol 2007; 102: $2667-2677$

[542] Homs MY et al. Esophageal stents with antireflux valve for tumors of the distal esophagus and gastric cardia: a randomized trial. Gastrointest Endosc 2004; 60: 695-702

[543] Shim CS et al. Management of malignant stricture of the esophagogastric junction with a newly designed self-expanding metal stent with an antireflux mechanism. Endoscopy 2005; 37: 335-339

[544] Wenger $U$ et al. An antireflux stent versus conventional stents for palliation of distal esophageal or cardia cancer: a randomized clinical study. Surg Endosc 2006; 20: 1675-1680

[545] Nagaraja V, Cox MR, Eslick GD. Safety and efficacy of esophageal stents preceding or during neoadjuvant chemotherapy for esophageal cancer: a systematic review and meta-analysis. J Gastrointest Oncol 2014; 5: $119-126$

[546] Mariette C et al. Self-expanding covered metallic stent as a bridge to surgery in esophageal cancer: impact on oncologic outcomes. J Am Coll Surg 2015; 220: 287 - 296

[547] Bergquist $\mathrm{H}$ et al. Stent insertion or endoluminal brachytherapy as palliation of patients with advanced cancer of the esophagus and gastroesophageal junction. Results of a randomized, controlled clinical trial. Dis Esophagus 2005; 18: 131 - 139

[548] Wenger $U$ et al. Health economic evaluation of stent or endoluminal brachytherapy as a palliative strategy in patients with incurable cancer of the oesophagus or gastro-oesophageal junction: results of a randomized clinical trial. Eur J Gastroenterol Hepatol 2005; 17: 1369 1377

[549] Bergquist $\mathrm{H}$ et al. Combined stent insertion and single high-dose brachytherapy in patients with advanced esophageal cancer-results of a prospective safety study. Dis Esophagus 2012; 25: 410-415

[550] Carazzone A et al. Endoscopic palliation of oesophageal cancer: results of a prospective comparison of $\mathrm{Nd}$ :YAG laser and ethanol injection. Eur J Surg 1999; 165: $351-356$
[551] Spencer GM et al. Laser augmented by brachytherapy versus laser alone in the palliation of adenocarcinoma of the oesophagus and cardia: a randomised study. Gut 2002; 50: 224-227

[552] Norberto L et al. Endoscopic palliation of esophageal and cardial cancer: neodymium-yttrium aluminum garnet laser therapy. Dis Esophagus 1999; 12: $294-296$

[553] Loizou LA et al. A prospective comparison of laser therapy and intubation in endoscopic palliation for malignant dysphagia. Gastroenterology 1991; 100: $1303-1310$

[554] Carter R, Smith JS, Anderson JR. Laser recanalization versus endoscopic intubation in the palliation of malignant dysphagia: a randomized prospective study. Br J Surg 1992; 79: 1167 - 1170

[555] Barr $\mathrm{H}$ et al. Prospective randomised trial of laser therapy only and laser therapy followed by endoscopic intubation for the palliation of malignant dysphagia. Gut 1990; 31: $252-258$

[556] Manner $\mathrm{H}$ et al. The tissue effect of second generation argon plasma coagulation (VIO APC) in comparison to standard APC and Nd:YAG laser in vitro. Acta Gastroenterol Belg 2007; 70: 352 - 356

[557] Eickhoff A et al. Prospective nonrandomized comparison of two modes of argon beamer (APC) tumor desobstruction: effectiveness of the new pulsed APC versus forced APC. Endoscopy 2007; 39: 637-642

[558] Manner $\mathrm{H}$ et al. Prospective evaluation of a new high-power argon plasma coagulation system (hp-APC) in therapeutic gastrointestinal endoscopy. Scand J Gastroenterol 2007; 42: 397-405

[559] Rupinski M et al. Randomized comparison of three palliative regimens including brachytherapy, photodynamic therapy, and APC in patients with malignant dysphagia (CONSORT 1a) (Revised II). The American journal of gastroenterology 2011; 106: 1612-1620

[560] Dempster $\mathrm{M}$ et al. Psychological distress among survivors of esophageal cancer: the role of illness cognitions and coping. Dis Esophagus 2012; 25: $222-227$

[561] Djärv T, Lagergren P. Six-month postoperative quality of life predicts long-term survival after oesophageal cancer surgery. European journa of cancer 2011; 47: 530-535

[562] Heijl M et al. Preoperative and Early Postoperative Quality of Life Predict Survival in Potentially Curable Patients with Esophageal Cancer. Annals of Surgical Oncology 2010; 17: $23-30$

[563] Gockel I et al. Long-term survivors of esophageal cancer: Diseasespecific quality of life, general health and complications. Journal of Surgical Oncology 2010; 102: 516-522

[564] Anstee $S$ et al. Developing a matrix to identify and prioritise research recommendations in HIV prevention. BMC Public Health 2011; 11: 381

[565] Herschbach P, Weis J. Screeningverfahren in der Psychoonkologie. Testinstrumente zur Identifikation betreuungsbedürftiger Krebspatienten. Dt. Krebsgesellschaft. 2010 http://www.krebsgesellschaft.de/ download/pso_broschuere.pdf

[566] Mehnert A et al. Assessment of psychosocial distress and resources in oncology - a literature review about screening measures and current developments. Psychother Psychosom Med Psychol 2006; 56: 462 479

[567] Mitchell AJ. Short screening tools for cancer-related distress: a review and diagnostic validity meta-analysis. J Natl Compr Canc Netw 2010; 8 $487-494$

[568] Vodermaier A, Linden W, Siu C. Screening for emotional distress in cancer patients: a systematic review of assessment instruments. J Natl Cancer Inst 2009; 101: 1464-1488

[569] Vodermaier A, Millman RD. Accuracy of the Hospital Anxiety and Depression Scale as a screening tool in cancer patients: a systematic review and meta-analysis. Support Care Cancer 2011; 19: 1899-1908

[570] Weis ], Schumacher A, Blettner G. Psychoonkologie: Konzepte und Aufgaben einer jungen Fachdisziplin. Der Onkologe 2007; 13: 185 194 
[571] Faller $\mathrm{H}$ et al. Effects of psycho-oncologic interventions on emotional distress and quality of life in adult patients with cancer: systematic review and meta-analysis. J Clin Oncol 2013; 31: 782 - 793

[572] Ärztliches Zentrum für Qualität in der Medizin (Hrsg.). Kompendium Q-M-A. 3. überarbeitete und erweiterte Auflage. Köln: Deutscher Ärzte-Verlag. 2008

[573] Donabedian A. Evaluating the quality of medical care. Milbank Mem Fund Q 1966; 44: 166-206

[574] Ärztliches Zentrum für Qualität in der Medizin (ÄZQ), Manual Qualitätsindikatoren. 2009. äzq Schriftenreihe: Berlin
[575] Vlayen J et al. Quality indicators for the management of upper gastrointestinal cancer - synthesis. Good Clinical Practice (GCP). Brussels: Belgian Health Care Knowledge Centre (KCE). 2013. KCE Reports 200 Cs. D/2013/10.273/16

[576] Walters DM et al. Understanding mortality as a quality indicator after esophagectomy. Ann Thorac Surg 2014; 98: 506-511; discussion 511-512

[577] Talsma AK et al. The 30-day versus in-hospital and 90-day mortality after esophagectomy as indicators for quality of care. Ann Surg 2014; 260: $267-273$

\section{ERRATUM}

S3-Leitlinie - Diagnostik und Therapie der Plattenepithelkarzinome und Adenokarzinome des Ösophagus

Porschen R, Fischbach W, Gockel I et al. S3-Leitlinie - Diagnostik und Therapie der Plattenepithelkarzinome und Adenokarzinome des Ösophagus. Z Gastroenterol 2019; 57: 336-418. DOI 10.1055/a-0833-5712

In der oben genannten Leitlinie wurden die Kollaboratoren wie folgt ergänzt:

Gustavo Baretton, Christian Ell, Ute Goerling, Lars Grenacher, Stephan Hollerbach, Barbara Kade, Wolfram Trudo Knoefel, Jürgen Körber, Philipp Lenz, Florian Lordick, Dietmar Lorenz, Sylvie Lorenzen, Alexander Meining, Josef Menzel, Helmut Messmann, Hans-Joachim Meyer, Stefan Paul Mönig, Ute Nöthlings, Heinz Schmidberger, Matthias Schmidt, Thomas Seufferlein, Maria Steingräber, Martin Stuschke, Reina Tholen, Jörg Trojan, Christoph Wagener, Arved Weimann, Frederick Wenz, Martin Werner 